

\section{Propositions}

Accompanying PhD thesis "Energy-based and biomimetic robotics."

1. Spineless biomimetic cheetah robots are a slight to nature.

2. A geometry-based look at synchronisation allows defining synchronicity and phase difference of arbitrary periodic systems.

3. The dynamic effect of a running cheetah's spine can be represented well by a single compliant element.

4. It is a welcome change to have a working prototype but no idea how it works, rather than a good theory but no functional prototype.

5. A new cost function should be introduced in optimal control:

$$
J:=\max _{i \in[1, N]}\left(x_{i}^{2}+y_{i}^{2}\right) ; \quad x_{i}, y_{i} \in \mathbb{R}^{N} .
$$

Since it takes the maximum over squared terms, people may finally talk about the "most optimal" solution without reprisal.

6. A good $\mathrm{PhD}$ thesis does not only report and document, but also teach and entertain.

7. If universities were serious about their education duty, they would find ways to value, stimulate and reward good teaching.

8. "Defence" is not a synonym of "stage play"- - having worked on their topic for four years, the $\mathrm{PhD}$ candidate should be given the proverbial third degree.

9. Trying to keep track of the state of the world through the news is futile: by definition, news is high-pass filtered; state reconstruction is subject to severe integration errors.

10. Nothing beats enharmonic humor, the intersection of puns and music. That's why you visit an optician when you don't $\mathrm{D} b$. Er gaat niets boven enharmonische humor, de intersectie van woordgrappen en muziek. Daarom eten we gisperfis. 


\title{
Energy-based and biomimetic robotics
}

\author{
Geert Folkertsma
}

2017 
The research presented in this thesis has been carried out at the Robotics and Mechatronics group and CTIT institute at the University of Twente. CTIT is the Centre for Telematics and Information Technology. This work is part of the research programme "DISC Graduate Programme" with project number 022.002.025, financed by the Netherlands Organisation for Scientific Research.

\section{RNM. CTIT UNIERSITY NWO}

Title Energy-based and biomimetic robotics

Author ir. Gerrit Adriaan (Geert) Folkertsma

Identification

ISBN 978-90-365-4316-3

ISSN 1381-3617 (CTIT Ph.D. Thesis Series No. 17-426;

CTIT, P.O. Box 217, 7500 AE Enschede, The Netherlands)

DOI $10.3990 / 1.9789036543163$

Cover design Geert and Iris Folkertsma (front, inside);

Bob Jansen, Ph.D (background) [74]

Printer Ridderprint BV, Ridderkerk

Fonts Equity (serif), Concourse (sans) [21] and Hack (monospaced) [135]

This thesis was typeset with $\mathrm{HTEX}_{\mathrm{E}}$; most images generated with the TikZ/pgf and pgfplots packages; it was maintained in a git/GitLab repository.

\section{$\$$ git $\uplus_{\text {Gitlab }} \quad$ Ti $k Z \quad \mathrm{LT}_{\mathrm{T}} \mathrm{X}$}

\section{Promotiecommissie}

Voorzitter en secretaris

prof. dr. P.M.G. Apers, Universiteit Twente

Promotor

prof. dr. ir. S. Stramigioli, Universiteit Twente

Co-promotor

prof. dr. A.J. van der Schaft, Rijksuniversiteit Groningen

Leden

prof. dr. ir. D.M. Brouwer, PDEng, Universiteit Twente

prof. dr. ir. H.F.J.M. Koopman, Universiteit Twente

prof. dr. B. Maschke, Université Claude Bernard Lyon 1

dr. A. Stokes, University of Edinburgh

prof. dr. A. Albu-Schäffer, Technische Universität München

(c) 2017 Geert Folkertsma 


\title{
ENERGY-BASED AND BIOMIMETIC ROBOTICS
}

\section{PROEFSCHRIFT}

\author{
ter verkrijging van \\ de graad van doctor aan de Universiteit Twente, \\ op gezag van de rector magnificus, \\ prof. dr. T.T.M. Palstra, \\ volgens besluit van het College voor Promoties \\ in het openbaar te verdedigen \\ op vrijdag 21 april 2017 om 16.45 uur \\ door
}

Gerrit Adriaan Folkertsma

geboren op 13 juni 1988

te Zwolle. 
Dit proefschrift is goedgekeurd door:

prof. dr. ir. S. Stramigioli, promotor

prof. dr. A. J. van der Schaft, co-promotor 
And I bet you, dear, a penny, You say mani-(fold) like many, Which is wrong. Say rapier, pier, Tier (one who ties), but tier.

-dr. Gerard Nolst Trenité, "The Chaos" (excerpt) 



\section{Summary}

The cheetah is a truly wondrous beast: to catch its prey it hunts them down at speed and runs at sixty $\mathrm{km}$-h at least!

A stunning figure-nature's best, indeed.

A legged robot has not yet come close.

They stumble at a low velocity, or if one ever to the same speed rose, its lack of grace was an atrocity.

For cheetah's high performing gaits we yearn, to be so fast, efficient, full of grace.

From nature therefore we must try to learn, to reap from evolution's high-speed race.

This plan makes sense, the logic sounds so smart!

The only problem is now: where to start?

The world around us runs on energy:

the "lingua franca" 'cross domains of sorts.

So $E$ in this work features centrally and interactions go through power ports.

This holds not solely for the physical, the presentation of the real-life thing, but also $\mathrm{E}$ is used for virtual:

describing algorithms, like a spring.

Control of port behaviour is the key. The flow and effort, always as a pair, determine interaction properly, as introduced by Hamilton of Éire.

This holistic view sounds splendid; yet will this give us a working robot pet? 
A running cheetah's butt goes down and upthat is to say: the shoulder and the hip.

So, looking at the motion in close-up, two hoppers are the legs that jump and skip.

To replicate this with efficiency, in resonance-based running like a boss, avoiding energy-deficiency, we have to minimise the impact loss.

A good mechanics analytical description of the loss's cause and source has shown which aspects here are critical and taught which rules of thumb we must enforce.

Alas, the final prototype that hopped did overheat: it stumbled and then stopped.

A running cheetah has a moving spine: it arches and it bends without a pause. This fact is surely an important sign; the cheetah does it for a certain cause.

Suppose that all this effort of the back in energy is nett conservative: a spring that alternates 'tween taut and slack; an oscillation that's repetitive.

Connect a pair of hoppers through a spring, then vary geometric properties: its stiffness, RCC, amount of swing, within some realistic boundaries.

And yes: with good parameters in place, the model starts to run! - if not yet race. 
A running cheetah shows quite readily that bodies front and rear in lockstep move.

The next pursuit is: study synchrony and hopefully our prototype improve.

The question first is: what is "synchronous"?

How "difference in phase" should be expressed?

Geometry is advantageous

defining terms like this, we can attest.

While homeomorphism-maps are by themselves still insufficient for control, a tube around the limit cycle, whyprovides an easy way towards the goal.

And now two Van der Pols are synchronised; hence thereby the approach legitimised!

We've studied all the aspects thoroughly, meticulously covered all the parts.

Described their functions quite exhaustively, so now the synthesis in earnest starts.

Another look at cheetahs videoed, their quantitive analysis complete, the final outcome of this study showed us how we can achieve our high-speed feat.

The spine reduced to simple 1-DoF joint equipped with springs, position movable, joined to a pair of legs - the case in point: it runs! Success is irrefutable.

So just a carper would point out right now the gait resembles rabbit hops somehow. 
Enough about the cheetah prototype; the legged locomotion is now done. Let's focus on a flying archetype: our research on the "Robird" has begun.

An ornithopter flies with flapping wings, exactly like a bird: no jet or prop.

Two heaving, pitching, twisting, beating limbs, in gliding mode can too be made to stop.

To other birds this robot looks just like the FALCO PEREGRINUS bird of prey. In order to prevent an airplane strike, it herds and drives all other birds away.

This makes for a successful enterprise, we just don't understand yet how it flies.

Some four years after starting on this quest, to study nature for robotics sake, we've put ideas and methods to the test. What did we learn in this; what did we make?

We've looked at energetic propertieslike port behaviour, power-routing lawsand how they change a robot's qualities: what positive behaviour they can cause.

A quantitative cheetah study has uncovered its dynamics, which has led to Cost OF TRANSPORT almost as good as the cheetah's gracious and efficient tread.

Continuing this potent research line, the "Robird" can expect a redesign. 


\section{Samenvatting}

\section{"Energiegebaseerde en biomimetische robotica"}

Een jachtluipaard of cheeta kan hard rennen: met een kruissnelheid van $60 \mathrm{~km} \mathrm{~h}^{-1}$ en een topsnelheid van $120 \mathrm{~km} \mathrm{~h}^{-1}$ is hij het snelste landdier ter wereld. Lopende robots zijn veel minder snel; bovendien gebruiken ze in vergelijking met het luipaard veel meer energie. In dit proefschrift wordt de loopbeweging van het jachtluipaard bestudeerd, om zo beter te begrijpen waar zijn goede prestaties vandaan komen. En, hopelijk, om die na te bootsen in een robot.

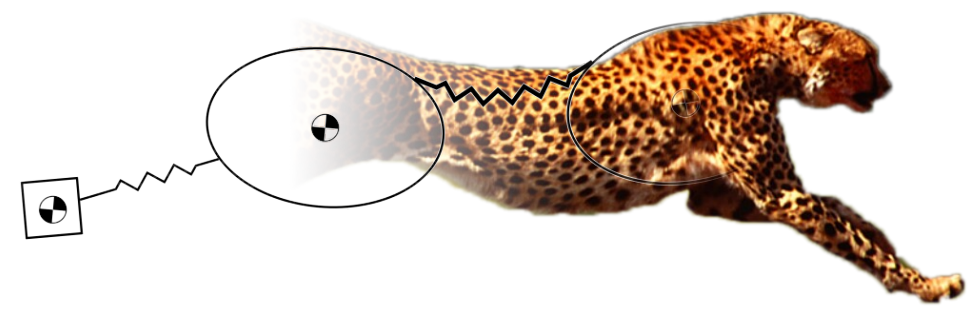

Het onderzoeksproject is getiteld "FREEQ: Fast-Running, Energy-Efficient Quadruped." Het doel is om geen ingewikkelde regelalgoritmes te bedenken, maar om juist de dynamica van de robot zo te maken dat hij zonder enige bijsturing al loopgedrag vertoont. Deze dynamica wordt afgeleid uit beelden van echte luipaarden, door te bestuderen hoe hij energie van zijn spieren omzet in voorwaartse beweging; hoe die energie wordt uitgewisseld tussen zijn poten en ruggengraat (potentiële energie) en zijn beweging (kinetische energie). 


\section{Energie in de robotica}

Bijna alle interacties tussen fysische systemen vinden plaats via uitwisseling van energie. In de theorie van poort-Hamiltoniaanse systemen staat de Hamiltoniaan, de totale energie in een (sub)systeem, centraal. Systemen kunnen aan elkaar gekoppeld worden met poorten waardoor vermogen wordt overgedragen, dus waar energie door wordt uitgewisseld.

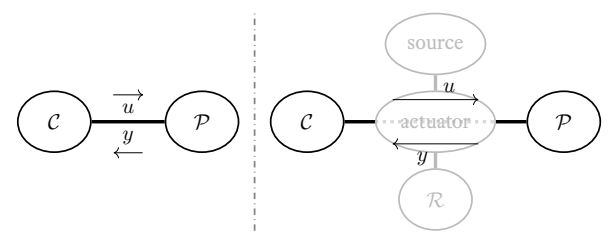

Zelfs regelalgoritmen kunnen beschreven worden als poort-Hamiltoniaans systeem. Een regelaar is dan niet meer een abstracte formule die een bepaald signaal probeert te sturen, maar wordt gekarakteriseerd door zijn interne energie en zijn gedrag aan de poort. Dit geeft inzicht in de energiestromen die tussen de regelaar $(\mathcal{C})$ en het systeem $(\mathcal{P})$ optreden.

\section{Efficiënt stuiteren}

Een luipaard heeft natuurlijk vier poten, maar als we er van de zijkant naar kijken, dus in het platte vlak, wordt het probleem wat eenvoudiger. Het betreft hier dus een tweebenige cheeta.
De poten van een jachtluipaard kunnen gezien worden als "hoppers." Het voor- en achterlijf stuiteren immers continu op en neer tijdens het rennen. We zouden dus een robot-cheeta kunnen maken door twee "hoppers" aan elkaar te verbinden met een ruggengraat.
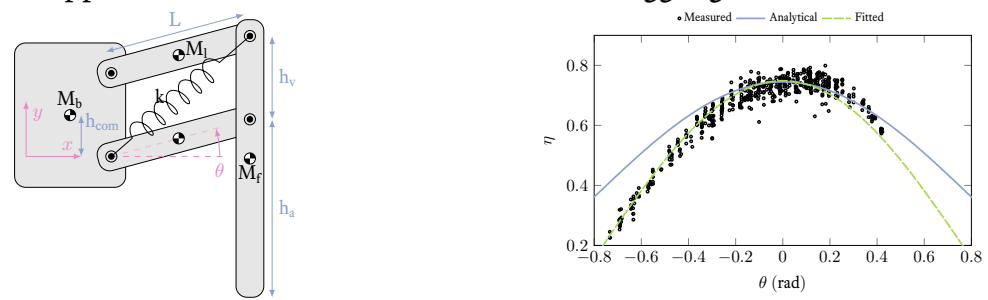

Om de robot efficiënt te maken, is het handig als de "hoppers" ook efficiënt zijn. Een uitgebreide studie naar verliezen tijdens het stuiteren leverde goede inzichten in de oorzaken van die verliezen.

\section{Flexibele ruggengraat}

De ruggengraat van een jachtluipaard buigt en strekt zich tijdens het rennen. Dit heeft gunstige effecten op zijn snelheid en efficiëntie, dus moet de robot-cheeta ook een ruggengraat krijgen. Om de robot zoveel mogelijk passieve dynamica te geven, bestuderen we het effect van een elastische ruggengraat. 

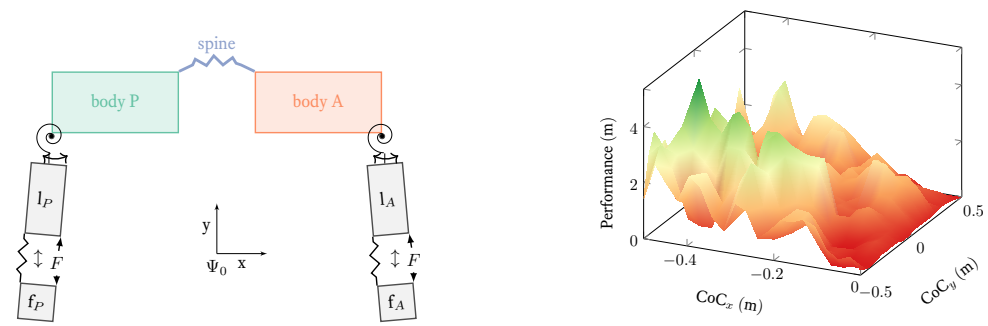

Door de geometrische eigenschappen van de ruggengraat te variëren, verandert het gedrag van het luipaardmodel. Het blijkt dat de locatie van het "Remote Centre of Compliance," dat punt waarop translatie- en rotatiestijfheden ontkoppeld zijn, van grote invloed is op het loopgedrag.

\section{Synchronisatie van periodieke systemen}

De "cheeta" met flexibele ruggengraat vertoont loopgedrag, maar dat is niet erg stabiel: hij struikelt vaak. Een echt jachtluipaard heeft een duidelijke tred of gang, waarbij de poten gesynchroniseerd bewegen. Om het loopgedrag van de robot te verbeteren, moeten de twee "hoppers" gesynchroniseerd worden, met een bepaald faseverschil tussen voor- en achterkant.
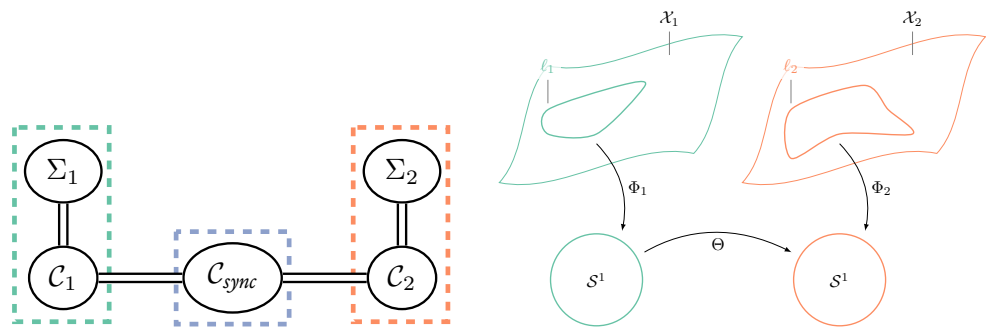

Wij definiëren "synchronisatie" op basis van geometrische afbeeldingen tussen de toestandsruimten van de systemen. Op deze manier is het mogelijk om eenduidig over synchronisatie en faseverschil te spreken. Willekeurige periodieke systemen zijn op deze manier met elkaar te synchroniseren.

\section{Synthese van een jachtluipaard-robot}

Met kennis over efficiënt stuiteren, de rol van de ruggengraat en synchronisatie tussen dynamische systemen, kan de volledige cheeta-robot gebouwd worden. De hoppers zijn geoptimaliseerd, de ruggengraat versimpeld tot een rotatieveer en de synchronisatie op basis van energieuitwisseling kan de tred stabiliseren. 

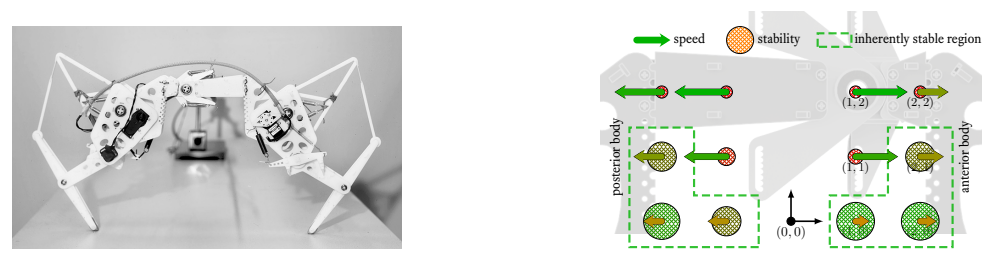

Het gebouwde prototype is volledig symmetrisch, op een uitzondering na: de ruggengraat, welke op verschillende locaties geplaatst kan worden. Met dit systeem is aangetoond dat deze simpele, tweebenige robot kan lopen, en dat de looprichting en -snelheid volledig veroorzaakt en bepaald worden door de asymmetrie van de ruggengraat!

\section{Slechtvalk "Robird"}

De jachtluipaard mag het snelste landdier zijn; in de lucht wordt hij afgetroefd door de slechtvalk: deze haalt snelheden van maar liefst $389 \mathrm{~km} \mathrm{~h}^{-1}$. De slechtvalk is het snelste dier ter wereld, alhoewel daarbij aangetekend moet worden dat hij dit alleen in duikvluchten haalt.

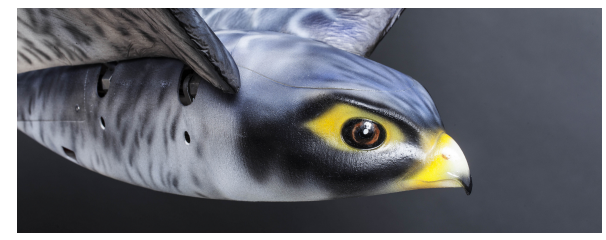

In het laatste hoofdstuk van dit proefschrift wordt aandacht besteed aan de "Robird," een robotvogel gebaseerd op de slechtvalk die vliegt door middel van vleugelslag.

\section{Zwarte marlijn}

De zwarte marlijn is het snelste zeedier ter wereld: hij kan $129 \mathrm{~km} \mathrm{~h}^{-1}$ zwemmen. Dit is erg indrukwekkend, en het ontwikkelen van een robot die dit ook kan zou zeer interessant zijn. Dit proefschrift heet echter niet "te land, ter zee en in de lucht." De marlijn komt verder dan ook niet voor in dit proefschrift.

Deze afbeelding komt van [22]

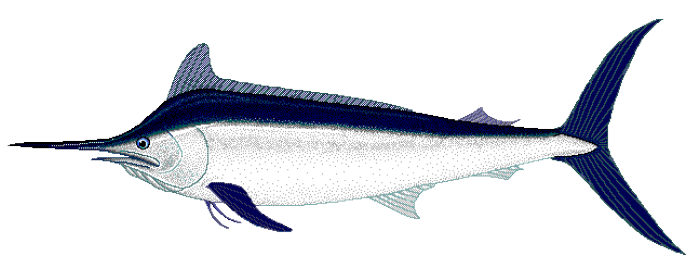




\section{Contents}

$\begin{array}{ll}\text { Colofon } & \text { II }\end{array}$

Summary

$\begin{array}{ll}\text { Samenvatting } & \text { XI }\end{array}$

Contents XV

1 Introduction 1

1.1 Biomimetic legged locomotion ........... 2

1.2 Morphological computation . . . . . . . . . . 2

1.3 The energy-based view .............. 3

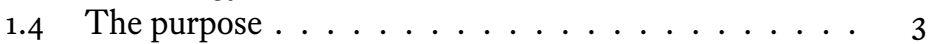

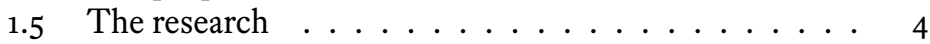

1.6 The starting point .................... 5

1.7 Theoretical framework ............. 6

1.8 What comes next ............ 6

2 Energy in robotics 9

2.1 Introduction . . . . . . . . . . . 10

2.1.1 Port-Hamiltonian systems . . . . . . 10

2.2 Energy in controlled physical systems . . . . . . 16

2.2.1 Passivity . . . . . . . . . . . 16

2.2.2 Energy and Distributed Architectures . . . . 18

2.2.3 Energy budgets . . . . . . . . . . . . 23

2.3 Control by interconnection . . . . . . . . . 26

2.3.1 Impedance control . . . . . . . . . . . 27

2.3.2 Energy shaping ............ 32

2.3 .3 Energy routing ............ 34

2.4 Control by physical interconnection . . . . . . . . . . 43

2.4.1 Physical compliance .......... 43

2.4.2 Variable stiffness ............. 49

2.4.3 Morphological computation ....... 56 
2.5 Conclusion .................. 58

3 Hoppers 59

3.1 Introduction . . . . . . . . . . 60

3.2 Design ........................ 61

3.2.1 Design Considerations .......... 61

3.2 .2 System ................ 62

3.3 Method ........................ 64

$3.3 .1 \quad$ Analytical .................. 64

3.3 .2 Simulation ........................ 66

3.3 .3 Real World . . . . . . . . . . . . . . 66

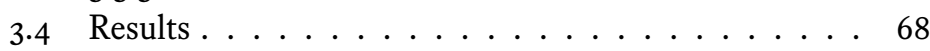

3.4 .1 Impact . . . . . . . . . . . 68

3.4 .2 Hopping ................ 69

3.5 Conclusion ................. 72

4 Spine 73

4.1 Introduction . . . . . . . . . . . 74

4.1.1 Geometrical bond-graph modelling . . . . . . 75

4.2 Model ..................... 75

4.2.1 Spine ...................... 77

4.2.2 Control ................ . . 79

4.3 Experiments ................ 81

4.3 .1 Simulation ................... 81

4.3 .2 Results .............. 84

4.4 Conclusion ............... 86

4.4.1 Conclusions ................ 86

4.4 .2 Future work ........... 86

5 Synchronisation $\quad 89$

5.1 Introduction . . . . . . . . . . 90

5.1.1 Synchronisation in dynamic locomotion . . . . 90

5.1.2 Related work ............. . . 91

5.1.3 Problem formulation .......... 92

5.2 Synchronisation ................... 93

5.2.1 Extension to off-limit cycle . . . . . . . . 95

5.2.2 Tangent maps . . . . . . . . . . . 96

5.2.3 Power-continuous synchronisation . . . . . . 98

5.2.4 Examples ......................... 98

5.3 Van der Pol oscillators . . . . . . . . . . . . . . 99

5.3.1 Energy-conservative Van der Pol . . . . . . . . 99

5.3.2 Influencing the limit cycle period . . . . . . 100

5.3.3 Synchronisation of two Van der Pol oscillators . 101

5.4 Circling masses . . . . . . . . . . . . 104 
5.4.1 Limit cycle control . . . . . . . . . 105

5.4.2 Synchronisation controller ........ 106

5.4.3 Synchronisation experiment ....... 109

5.5 Conclusion . . . . . . . . . . . 111

6 Synthesis 113

6.1 Introduction: on quadrupeds and the spine . . . . . . . 114

6.1.1 Morphological computation and the spine . . . 114

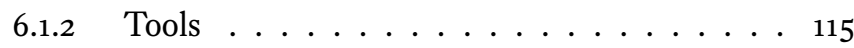

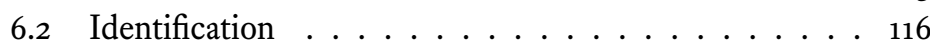

6.2.1 Video analysis . . . . . . . . . . . . 116

6.2.2 Observed principles: Twist . . . . . . . . . . 117

6.2.3 Observed principles: Wrench . . . . . . . . . 119

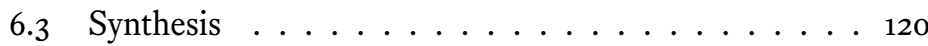

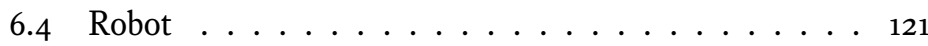

6.4.1 Control ... . . . . . . . . . . 124

6.4.2 Single-hopper experiments . . . . . . . . 124

6.5 Results . . . . . . . . . . . . . 125

6.5.1 Natural dynamics . . . . . . . . . . . 125

6.5.2 Phase stabilisation control . . . . . . . 127

6.5.3 Successful morphological computation . . . . . 127

6.6 Conclusion .................. 130

7 Robird 131

7.1 Introduction . . . . . . . . . . 132

7.1.1 Research perspective ........... 132

7.1.2 Other ornithopters ........... 133

7.1.3 Peregrine Falcon and Bald Eagle . . . . . . . 134

7.2 System . . . . . . . . . . . . . . 135

7.2.1 Wings and mechanism ......... 135

7.2.2 Control surfaces . . . . . . . . . . . . 135

7.2.3 Avionics . . . . . . . . . . . . . 136

7.2.4 Robustness . . . . . . . . . . . . 136

$7 \cdot 3$ Dynamic system model . . . . . . . . . . . 136

7.3.1 Fixed-wing Aerodynamics . . . . . . . . . 136

7.3.2 Flapping-wing aerodynamics . . . . . . . . . 137

7.3.3 Dynamics ............... 139

7.3.4 Wind tunnel measurements ....... 140

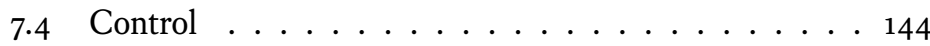

7.4.1 Flight modes . . . . . . . . . . . . . 144

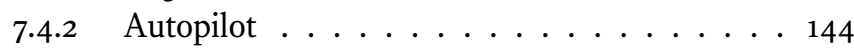

7.5 Conclusion . . . . . . . . . . . 147 
8 Conclusion 149

8.1 The answers . . . . . . . . . . . 150

8.1.1 The cheetah robot .......... 150

8.2 The implications . . . . . . . . . . . . 152

8.2.1 Quadrupedal robots ............ 152

8.2.2 Biomimetic robots . . . . . . . . . . 153

8.2.3 Energy-aware robots ........... 154

8.3 The new questions . . . . . . . . . . . . 155

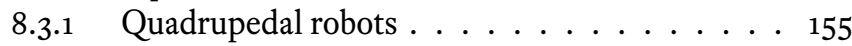

8.3.2 Biomimetic robots ........... 156

8.3 .3 Energy-aware robots ........... 156

8.4 Conclusion . . . . . . . . . . . . 157

$\begin{array}{ll}\text { References } & 159\end{array}$

Appendices

$\begin{array}{ll}\text { A Original research proposal } & 177\end{array}$

B Energy control: proof $\quad 185$

C Embedded, Energy-Aware Actuators $\quad 187$ 


\section{Chapter 1}

\section{Introduction}

Robotic research can learn from nature. When designing an artificial system - for example a robot-for a certain task, one can start from scratch when designing the system, dynamics and control laws. Or, one can first study nature, to see how millions of years of evolution have solved similar problems, and take these lessons as a starting point. This idea of biomimetic robotics sounds good, but its application may be difficult. In this thesis, we look at

how exactly to "study nature";

where to look at "similar problems";

what "these lessons" are that can be extracted; and in

which way these can be applied to robotics.

These questions are addressed in an energy-based framework: the thesis is about energy-based biomimetic robotics. We shall study methods and tools; design a cheetah robot and introduce a robotic falcon. 


\section{§1 Biomimetic legged locomotion}

Within biomimetic robotics, the focus of this work is on legged locomotion. Moving about on legs tends to be harder than rolling on wheels, as far as robots go. However, robotics researchers see enough advantages of legged locomotion over wheels. Consequently, much research is carried out on the topic, focussing on: bipeds, quadrupeds, hexapods; balancing, walking, running; speed, energy-efficiency, robustness...The motivation for this research is simply curiosity: how does legged locomotion work? Can the current state-of-the-art be improved? Are there more lessons to be learned in this field of research?

Be it because of versatility, or simply from a lack of grown rotational joints, most large land animals have legs. The advanced locomotion systems of these animals have been "developed" over millions of years of evolution. One can expect to find good inspiration for designing artificial walking machines-legged robots - by studying these biological systems. The fastest land animal is the cheetah: it is much more slender than other big cats (like the lion), relying more on speed than strength for hunting [67]. If a fast quadrupedal robot must be developed, the cheetah is a good source of inspiration.

When doing biomimetic robotics research, it is important to realise that nature and robots are quite different. The inspiration for this research, the cheetah, has many capabilities: it can jump, lie down, climb trees, and run fast. We are only interested in the part where it runs very fast. At the same time, robots offer different opportunities than a real cheetah: rotational joints, fast computers and absolute measurements are not found in nature. We are not bound by some of the restrictions that nature has. The important question is therefore: how to do "biomimetics" in such a way that we learn important lessons from nature, while taking advantage of the technology at our disposal? Specifically, in this thesis: how to make a biomimetic fast-running, energy-efficient quadruped?

\section{§2 Morphological computation}

In slowly-walking robots, the inertia of the mechanical system is often negligible: the stiffness of the mechanisms and control algorithms ensures that the lowest eigenfrequencies are far above the walking frequency. The feet may execute position-controlled trajectories that mimic a walking motion; stability control keeps the balance of the robot.

Conversely, in fast locomotion, the dynamics of the robot itself become important in design and control. The dynamics of the mechanical system and the dynamics of the controller together shape the motion 
of the robot. If the mechanical system is properly designed, much of the desired behaviour is already present in the inherent dynamics of the system. Control algorithms can excite, stabilise or augment these natural dynamics with little effort. This idea is called morphological computation, because much of the "computation" of "control actions" is carried out by the morphology. The morphology describes the mechanical properties of the system: mass distribution, compliance, mechanisms, et cetera.

\section{§3 The energy-based view}

There is no need to make a hard distinction between control algorithms and the physical system. Together they form the robot: both have their dynamics, and the behaviour of the robot is the result of their interconnection. This interconnection can be characterised by energy exchange. From the actuators into the robot, or from the robot, through the actuators, into the control algorithm. The dynamics of both subsystems can also be described in an energy-based way: in the mechanical system, energy is exchanged between storage elements such as springs and masses, transformed or dissipated, depending on the morphology. In Control by Interconnection, control algorithms are described by powercontinuous transformations or dissipation, by (virtual) storage elements and their interconnection - to each other and to the physical world - all determining the energy flow.

By describing, modelling and designing both the control algorithms and the mechanical system of the robot as energy-based systems, with power ports for interconnection, there is no conceptual distinction between control and mechanical system. There is just one holistic, energy-based view of the robot. Morphological computation-which is based on the proper interconnection of physical storage elements - and this energybased view - wherein storage elements, interconnection and energy flows are explicitly modelled-are a perfect match.

\section{$\S 4$ The purpose}

The purpose of the work presented in this thesis is to bring together Port-Hamiltonian System theory and the field of morphological computation. As motivated above, the similarities between this mathematical formulation on the one hand, and mechanical design ideas on the other hand, are striking. To connect them together in one study may lead to new insights. 
The cheetah robot is the central case study of this thesis. The fusion of morphological computation and the Port-Hamiltonian System theory enables "quantitative biomimetics": the dynamics and energetic behaviour of a running cheetah can be studied, abstract lessons can be extracted and subsequently applied in a mechanical cheetah robot. The result is not only a running quadrupedal robot, but also the methodology that enables the design and realisation of this robot.

The contributions of this work will be threefold:

1. There are many quadrupedal robots being developed and researched around the world. Lessons learned in the design of our cheetah may be beneficial to others. Because of our unique approach, based on dynamics, energy and morphological computation, these lessons are expected to be an enrichment for the field of quadrupedal locomotion.

2. We design the cheetah robot using "quantitative biomimetics," studying the dynamics and energetic behaviour of a real cheetah. This method is not restricted to the cheetah, to the field of quadrupedal robots, or even to legged locomotion. Hopefully, the methodology can be applied by other researchers who want to take inspiration from dynamics in nature.

3. Finally, the various tools and aspects of the methodology shall not be restricted to biomimetics or walking robots alone. The practical application of energy-based control laws, control-byinterconnection, or dynamic morphological computation can be used in any kind of robotics research.

\section{$\S 5$ The research}

The research is focussed on the development of a cheetah robot. The main, motivating research question is therefore:

\section{"What are the important dynamic functions in a running cheetah? "}

This leads to some other questions:

1. How can these functions be identified?

2. Can they be identified in a quantitative manner?

3. What tools and methods are needed to describe them?

4. How can the essential functions be synthesised into the design of a robot? 


\section{§6 The starting point}

The inspiration for this work came from working on the "MITCheetah" ([130]). The research, presented in [45], focussed on the role of a flexible spine and parallel stiffness in a quadrupedal robot. Although the outcome of the study was very positive, showing greatly reduced energy consumption from the springs and spine, the research raised many questions, too:

- The MITCheetah is a biomimetic robot, designed by roughly copying measurements and joint locations from the real cheetah. Is this the best way to mimic the desired behaviour of high-speed locomotion?

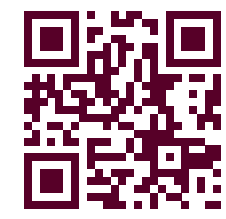

MIT Cheetah running in simulation

- The flexible spine, consisting of rigid vertebrae and soft intervertebral discs, looks very much like a real spine. Is this complexity required for its function?

- In subsequent work on the MITCheetah the spine was locked, because it made control problems harder, rather than easier. How can a spine be used to actually improve the behaviour, without adding much complexity to the system?

These questions resulted in the proposal in Appendix A, with the main aim to do dynamic biomimetics with a focus on the role of the spine, where the desired dynamic behaviour is copied, rather than the morphology itself.

An initial, quantitative study of running cheetahs resulted in three main "dynamic aspects" that can be identified:

Spine Many quadrupedal robots do not have a spine, but consist of a rigid body with four legs. However, a galloping cheetah arches and bends its back during the stride. Clearly, this has a large influence on the dynamics of the running cheetah.

Hopping With a focus on the spine, the cheetah can be seen as an anterior body (or body part) and a posterior body, connected by the spine. These two bodies have legs that provide necessary upward and forward momentum to keep running, by pushing off from the ground.

Synchronisation The way a cheetah runs can be qualified using gait patterns, which describe the order in which the feet touch the ground. Clearly, there is some sort of synchronisation between the legs, which keeps the cheetah running in a certain gait.

These three aspects will be studied in more detail in this thesis. 


\section{$\S 7$ Theoretical framework}

Central to the analysis and design of the cheetah robot is the energybased approach. A thorough introduction to this approach is presented Chapter 2: Energy in robotics. Bond graphs are used to visualise the system models (see Paynter [98] and Breedveld [17]). The storage elements of bond graphs can be directly related to the Hamiltonian of Port-Hamiltonian System (PHS) equations; resistive elements to the dissipation matrix. All power-preserving interconnections in bond graphs, such as transformers and the $\mathbf{o}$ and $\mathbf{1}$-junctions, represent the Dirac structure or the skew-symmetric J-matrix of the PHS formulation.

It will become clear, in the chapters concerning morphological computation and synchronisation, that this graphical bond graph expression gives further insight into the interconnection and energy flows.

Finally, Lie Group theory will be used for the geometric description of robot dynamics.

\section{$\S 8$ What comes next}

Before focussing on the cheetah, the energy-based view, methods and tools will be extensively introduced in Chapter 2: Energy in robotics. Then, although the main focus of the biomimetic morphological computation is the spine, a means of propulsion or actuation is required before the spine can be tested in a cheetah model. Therefore, the first aspect of the cheetah that is studied is the hopping, in Chapter 3: Hoppers. Because these "hoppers" will have to function as the legs of a resonancebased dynamic morphological computation cheetah, special attention is

* Although a spatial spring does not have "Degrees of Freedom" in the traditional sense of the word, 6-DoF here means compliance in all three translational and rotational directions. given to the energy-efficiency of the hopping.

Then: the spine. From previous work on the MITCheetah (Folkertsma, Kim and Stramigioli [45]), an elastic spine was found to be beneficial. The effect of a fully compliant, 6-DoF spatial spring* as spine is explored in Chapter 4: Spine. The result of this chapter is a cheetah model that shows locomotion behaviour induced by the spine, but the gait is not very stable.

If the anterior and posterior hoppers can be synchronised, the gait is stabilised and the locomotion behaviour induced by the compliant spine may improve. Therefore, in Chapter 5: Synchronisation, synchronisation of periodic motions - of limit cycles - is studied. 
With all these parts in place, we have set a direction for:

How to study nature: in an energy-based way.

Where to look in the cheetah: at the motion of the anterior and posterior bodies, connected by the spine.

What lessons to extract: the dynamics of the spine.

Which way to apply these on a cheetah robot: energy-efficient, resonancebased hoppers connected by a compliant spine.

In Chapter 6: Synthesis, the quantitative biomimetic study is carried out; important lessons for the design of an artificial spine are extracted and a cheetah robot is designed, built and tested. It shows successful, stable, dynamic morphological computation.

The synthesis chapter concludes the work on the cheetah. However, we are also working on another biomimetic robot: the "Robird," a robotic falcon. We are starting to apply the methods and tools used and developed in the cheetah research also on the Robird. Although this is mostly future work, the robotic peregrine falcon is introduced in Chapter 7: Robird.

Some of the methods can be applied directly, for example to study and control the synchronisation of the beating wings, or in analysing and optimising the energy-efficiency of the driving mechanism. Other aspects of the Robird are fundamentally different: aerodynamic forces and wing deformation are both distributed-parameter phenomena, something that has not been studied for the cheetah. There is also a difference in approach: the Robird already exists, and flies: it does not have to be designed. We want to understand it-at least better than we do now. 
Chapter 1

Introduction 


\section{Chapter 2}

\section{Energy in robotics}

Energy and energy exchange govern interactions in the physical world. By explicitly considering the energy and power in a robotic system, many control and design problems become easier or more insightful. We show the application of these energy considerations to robotics; starting from the fundamental aspects, but, most importantly, continuing to the practical application to robotic systems. Using the theory of Port-Hamiltonian System as a fundamental basis, we show examples concerning energy measurement, passivity and safety. Control by interconnection covers the shaping and directing of energy inside the controller algorithms, to achieve desired behaviour in a power-consistent manner. This idea of control over the energy flows is extended to the physical domain. The boundary between controller and robot disappears and everything is an interconnected system, driven by energy exchange between its parts.

This chapter will appear as a tutorial, article and book in "Foundations and Trends ${ }^{\circledR}$ in Robotics" by now Publishers Inc. 


\section{$\S 1$ Introduction}

The physical world is governed by energy.

From the kinetic energy in a speeding car to the first law of thermodynamics, energy is the lingua franca in all physical domains. It is a coordinate-independent description of the energetic state of a system.

Interactions are almost exclusively ${ }^{1}$ characterised by energy exchange.

From a battery, through an electric motor-via the magnetic fields - to the mechanical system of a robot: the power or exchanged energy can be traced across all these physical domains. A car does not speed up because the engine applies a torque on the wheels: that is simply the means of pouring energy from the petrol or battery into the kinetic energy of the car as a moving mass.

Many applications in robotics are concerned with energy: the amount of kinetic energy in the robot (e.g. for safety issues), a periodic motionoscillation - with a certain amplitude (i.e. total energy), energy-efficiency objectives, and storing and releasing energy in springs for explosive motions are some examples.

By considering the energy in robotic systems explicitly, more insight can be gained, control problems may become easier and a "feel" for the actual physical processes emerges. This energy-based perspective should not focus on only the control system, nor should it only focus on the description of the physical robot. We present a holistic, energy-based view of robotic systems: Energy in Robotics. To achieve this holistic view, we shall address the following topics:

1. Energy-based formulation of physical systems: Port-Hamiltonian System theory.

2. Passivity and stability in robotic systems.

3. Measurement and control of energy flowing through actuators.

4. Energy-based controller design: energy shaping and energy routing in the controller.

5. Energy-based system design: shaping the energy flows in a physical robotic system.

\section{$\S 1.1$ Port-Hamiltonian systems}

Hamiltonian mechanics is a theory of classical mechanics similar to Lagrangian mechanics. The classical canonical formulation is described

${ }^{1}$ Certain interactions, like ideal constraints, can influence motion without energy exchange. 
by a set of equations governing the Hamiltonian:

$$
\begin{aligned}
\frac{\mathrm{d} \mathbf{p}}{\mathrm{d} t} & =-\frac{\partial \mathcal{H}}{\partial \mathbf{q}} \\
\frac{\mathrm{d} \mathbf{q}}{\mathrm{d} t} & =+\frac{\partial \mathcal{H}}{\partial \mathbf{p}} \\
\mathcal{H} & =T+V .
\end{aligned}
$$

$\mathcal{H}$ is the Hamiltonian, the sum of kinetic $T$ and potential energy $V$, i.e. the total internal energy of the system; $\mathbf{q}$ and $\mathbf{p}$ are the generalised coordinates and momenta, respectively. It is clear that Hamiltonian mechanics is very suitable for energy-based modelling and control: the total energy is expressed explicitly in the equations.

Example 2.1. A simple example of a physical system described with Hamiltonian mechanics is the mass-spring oscillator. The position $\mathbf{q}$ is the spring deflection; momentum $\mathbf{p}$ is the momentum of the mass, $\mathbf{p}=m \cdot v$. With kinetic energy $T=p^{2} /(2 m)$ (mass $m$ ) and potential energy $V=q^{2} /(2 C)$ ( $C$ is the compliance of the spring, the inverse of its stiffness) the dynamic equations become:

$$
\begin{aligned}
\mathcal{H} & =\frac{p^{2}}{2 m}+\frac{q^{2}}{2 C} \\
\frac{\mathrm{d} p}{\mathrm{~d} t} & =-\frac{q}{C} \\
\frac{\mathrm{d} q}{\mathrm{~d} t} & =\frac{p}{m} .
\end{aligned}
$$

Of course, in the equation for $p$ we recognise $\dot{p}=F$, Newton's second law; in this case $m \dot{v}=K q$. The equation for $q$ is the obvious $\dot{q}=v$.

$\langle$ example end $\rangle$

This example shows that energy is explicitly modelled: when solving the equations one will see the energy flow between $T$ and $V$. In this closed system without friction, the total energy $\mathcal{H}$ is of course conserved.

In robotics, however, there is always interaction: between mechanical parts, across domains through transducers, and with the environment. For this interaction, the sub-systems must be interconnected. This interconnection can be described by so-called power ports: interfaces that transfer energy between elements, domains, systems. A power port is always a pair of variables whose pairing characterises the power exchange, e.g. force and velocity or voltage and current.

In port-Hamiltonian systems theory, a common representation is the causal Poisson framework representation, which is an input-state-output representation. In this representation, all the states like $q$ and $p$ are 
collected in a single state vector which may even be a combination of generalised moments and displacements and indicated as $x$ :

$$
\begin{aligned}
\dot{x} & =[J(x)-R(x)] \frac{\partial \mathcal{H}}{\partial x}(x)+g(x) u & & x \in \mathcal{X}, u \in \mathbb{R}^{m} \\
y & =g^{\top}(x) \frac{\partial \mathcal{H}}{\partial x}(x), & & y \in \mathbb{R}^{m}
\end{aligned}
$$

where $J(x)=-J^{\top}(x), R(x)=R^{\top}(x) \geq 0 . J$ is an internal interconnection matrix; $R$ is a resistive structure. $g$ represents the interconnection, and therefore effect, of the port variables on the state variables and viceversa.

The matrix $J$ is a power-continuous interconnection by its skew-symmetry, whereas $R$ models pure resistive losses of the system, as can be seen by taking the time derivative of the Hamiltonian:

$$
\begin{aligned}
\dot{\mathcal{H}}(x) & =\frac{\partial \mathcal{H}^{\top}}{\partial x}(x) \cdot \dot{x} \\
& =\frac{\partial \mathcal{H}^{\top}}{\partial x}(x)[J(x)-R(x)] \frac{\partial \mathcal{H}}{\partial x}(x)+\frac{\partial \mathcal{H}^{\top}}{\partial x}(x) \cdot g(x) u \\
& =-\frac{\partial \mathcal{H}^{\top}}{\partial x}(x) R(x) \frac{\partial \mathcal{H}}{\partial x}(x)+y^{\top} u
\end{aligned}
$$

which is the power supplied through the port $y^{T} u$, minus the power lost to friction, quadratic on $R(x)$.

Example 2.2. Consider the mass-spring-damper system in Figure 2.1: it does not have an external interaction port, so $g(x) \equiv 0$, hence the Hamiltonian should change only with the quadratic $R(x)$ term of (2.4). The

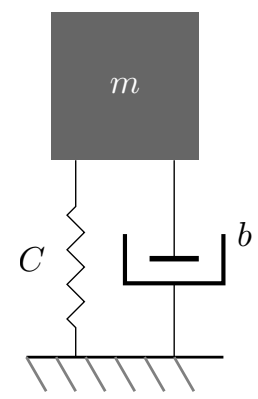

Figure 2.1 - A mass-spring-damper system. (Example 2.2)

state vector comprises $p$ and $q$ as in Example 2.1; the damping force 
$F_{\mathrm{b}}=b \cdot v=b \cdot p / m$ is modelled in the $R$ matrix.

$$
\begin{aligned}
\mathcal{H}(x) & =\frac{p^{2}}{2 m}+\frac{q^{2}}{2 C} \\
\left(\begin{array}{c}
\dot{p} \\
\dot{q}
\end{array}\right) & =\left[\left(\begin{array}{cc}
0 & -1 \\
1 & 0
\end{array}\right)-\left(\begin{array}{ll}
b & 0 \\
0 & 0
\end{array}\right)\right]\left(\begin{array}{l}
p / m \\
q / C
\end{array}\right)
\end{aligned}
$$

Figure 2.2 shows a simulation of this example system, with $C=1 \mathrm{~m} \mathrm{~N}^{-1}, b=$ $0.1 \mathrm{~N} \mathrm{~s} \mathrm{~m}^{-1}, m=1 \mathrm{~kg}, x(0)=\left(\begin{array}{ll}0 & 1 \mathrm{~m}\end{array}\right)^{\top}$. Especially the plot of the energy shows how the Hamiltonian $\left(E_{M}+E_{C}\right)$ decreases with the energy dissipated in the damper, as expected from (2.4). ( $E_{\mathrm{M}}$ and $E_{\mathrm{C}}$ are the first and second term of the Hamiltonian of $(2.5) ; E_{\mathrm{R}}$ is the energy dissipated by the damper, given by $\int F_{\mathrm{b}} v \mathrm{~d} t=\int b v^{2} \mathrm{~d} t$.)

$\langle$ example end $\rangle$

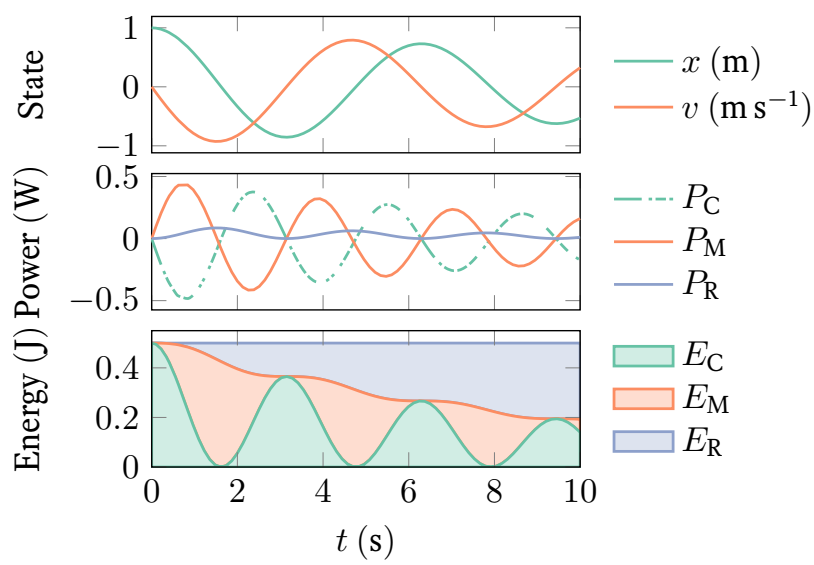

Figure 2.2 - Simulation of the mass-spring-damper system of Figure 2.1. Energy flows back and forth between the spring and mass, and is dissipated in the damper. (Example 2.2)

Example 2.3. An example of a system with an external port is the sliding mass, with an actuator applying a force on it, as in Figure 2.3. The only state is the momentum $p$. Choosing $F$ as the input determines $g(x)=1$ and the dynamic equations are:

$$
\begin{aligned}
\mathcal{H}(x) & =\frac{p^{2}}{2 m} \\
\dot{x}=\dot{p} & =[(0)-(b)] \cdot \frac{p}{m}+(1) F \\
y & =(1)^{\top} \frac{p}{m} .
\end{aligned}
$$




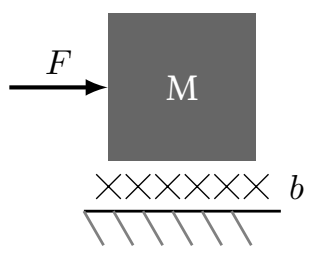

Figure 2.3 - A mass sliding on a surface with friction, with a port to the environment: the actuator force. (Example 2.3)

The choice for $F$ as input has made $y=p / m=v$, such that the product of input and output is power and this is indeed a power port. Simulation results

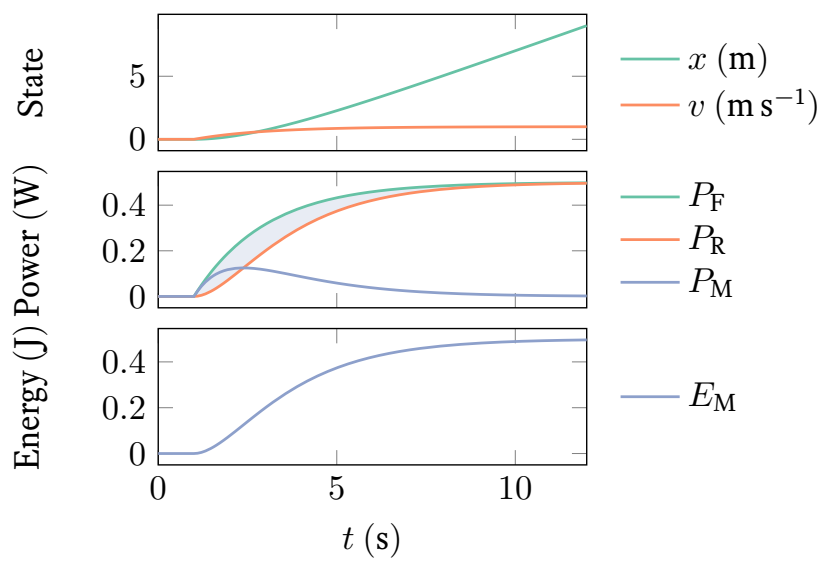

Figure 2.4 - Simulation of the sliding mass in Figure 2.3. The difference between the power supplied through the port, $P_{\mathrm{F}}$, and the power lost to friction, $P_{\mathrm{R}}$, is equal to the time derivative of the Hamiltonian $E_{\mathrm{M}}$. (Example 2.3)

of this system (with $m=1 \mathrm{~kg}, b=0.5 \mathrm{~N} \mathrm{~s} \mathrm{~m}^{-1}, F=0.5 \mathrm{~N} 1(t-1)$ ) are shown in Figure 2.4. The difference between the power injected by the actuator $\left(P_{\mathrm{F}}=v \cdot F\right)$ and the power lost in friction $\left(P_{\mathrm{R}}=b v^{2}\right)$, shaded in the middle graph, is exactly equal to the time derivative of the Hamiltonian, $\dot{E}_{\mathrm{M}}=P_{\mathrm{M}}$.

$\langle$ example end $\rangle$

Finally, the port of the Port-Hamiltonian System is an interface: the system can be connected to other systems through this power port. The interconnection between two or more PHS is described by a Dirac structure, which is a power-continuous coupling of the port variables. In fact, the mass-spring-damper of Example 2.2 can be viewed - and modelledas three PHS, one for each element, interconnected by a Dirac structure, 
as in Figure 2.5. The interconnection of Port-Hamiltonian Systems is again a Port-Hamiltonian System, with a Hamiltonian that is the sum of the two systems' Hamiltonians and a new internal interconnection matrix $J$ that incorporates the (old, external) Dirac structure.

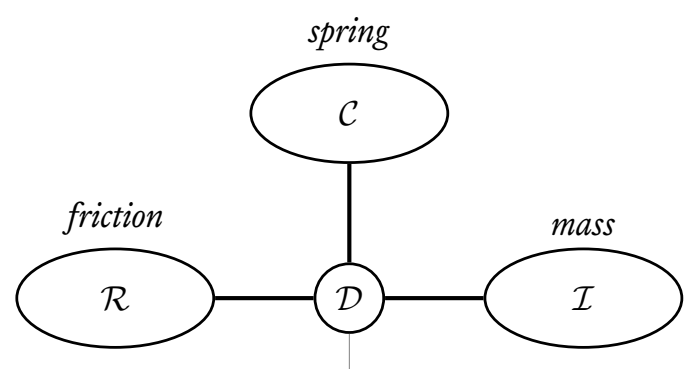

Dirac structure

Figure 2.5 - A Dirac structure is a power-continuous interconnection between Port-Hamiltonian Systems. This figure shows the system of Figure 2.1 as three interconnected elements, or systems.

An excellent introductory overview of Port-Hamiltonian Systems Theory can be found in [123]. 


\section{§2 Energy in controlled physical systems}

\section{$\S 2.1$ Passivity}

Willems [164] introduced the concept of dissipative dynamical systems as follows. Consider a dynamical system $\Sigma$ with state $x$, input $u$ and output $y$ and a real-valued function $w(u, y)$ called the supply rate. If a non-negative storage function $S(x)$ can be found such that:

$$
S\left(x_{0}\right)+\int_{t_{0}}^{t_{1}} w(u(t), y(t)) \mathrm{d} t \geq S\left(x_{1}\right)
$$

then the system is said to be dissipative.

When describing the dynamics of physical systems using Port-Hamiltonian Systems Theory, a natural choice for both $w$ and $S$ are $w(u, y)=y^{\top} u$ and $S(x)=\mathcal{H}(x)$. As seen before in (2.4):

$$
\dot{\mathcal{H}}(x)=y^{\top} u-\frac{\partial \mathcal{H}}{\partial x}^{\top}(x) R(x) \frac{\partial \mathcal{H}}{\partial x}(x) .
$$

This can be recognised as the differential form of (2.7): since $R=R^{\top} \geq$ 0 , the square term on the RHS is $\geq 0$. The storage function is the energy present in the system and the supply rate $y^{\top} u$ is the power transferred to the system through its port.

Physical systems for which the inequality holds are passive systems. If $R=0$, i.e. there is no dissipation ${ }^{2}$, then equality holds in (2.8) and the system is said to be conservative or lossless.

We refer to [122] for a unified and in-depth treatment of passivity and $L_{2}$-gain theory; dissipative systems; nonlinear stability; and other related subjects.

\section{Passivity as a must}

Many robots are controlled in a non-passive way, for example through PID joint controllers or non-passive state feedback. This leads to good performance, and generally stability can be proven for the free-standing robot. However, as soon as the robot interacts with its environment, things become completely different-especially if it is an unknown environment. In that case, it turns out that a passively-controlled robot is a necessary condition for stability, as shown in the following theorem, first presented in [23, $\left.\mathrm{Ch}_{3}\right]$.

\footnotetext{
${ }^{2}$ Properly speaking, energy cannot be dissipated for the first principle of thermodynamics, but what is meant here is what is called free energy and this means irreversible transformation of energy to heat.
} 
Theorem 2.2.1. Given a non-passive system $\Sigma$ with input-output pair $(u, y)$, then there always exists a passive system $\tilde{\Sigma}$ that, when connected to $\Sigma$, will give rise to unstable behaviour of the interconnection of $\Sigma$ and $\tilde{\Sigma}$.

Proof. By the definition of passivity, (2.7), non-passiveness of $\Sigma$ implies that $\exists \bar{u}(t)$ such that the integral of minus the supply rate is unbounded, which means we can extract infinite energy from the system. Indicate with $\bar{y}(t)$ the output corresponding to the input $\bar{u}(t)$. This means that we can define the extracted energy function $H_{o}(t)$ as:

$$
H_{o}(t)=\int_{0}^{t} \bar{u}(s) \cdot \bar{y}(s) \mathrm{d} s .
$$

By construction, $\lim _{t \rightarrow \infty} H_{o}(t)=\infty$. This implies that due to the continuity of $H_{o}(t), \exists$ a bounded $H_{\min }:=\min _{t} H_{o}(t)$

We will now constructively define a passive system $\tilde{\Sigma}$ that will generate the input $\bar{u}(t)$ as its output $\tilde{y}$ :

$$
\begin{aligned}
& \dot{x}=n(t) \tilde{u} \\
& \tilde{y}=n(t) \frac{\partial \tilde{H}}{\partial x},
\end{aligned}
$$

with $\tilde{H}(x)=\frac{1}{2} x^{2}$ and $n(t)=\frac{\bar{u}(t)}{\frac{\partial \tilde{H}}{\partial x}}$. It is easy to see that this system is passive (even conservative) with storage function $\tilde{H}(x)$. By initialising $x(0)=\sqrt{2 H_{\min }+\epsilon}$ for any $\epsilon>0$, it can be seen that by construction $\frac{\partial \tilde{H}}{\partial x}(t)>0 \forall t>0$. Therefore, it is always possible to calculate $n(t)$. By setting as interconnection $\tilde{u}=y$ and $u=\tilde{y}(=\bar{u})$, we by construction have that

$$
\lim _{t \rightarrow \infty} H_{0}(x)=\lim _{t \rightarrow \infty} \tilde{H}(x)=\infty \Rightarrow x \rightarrow \infty
$$

which proves instability of the coupled system, due to its diverging state $x$.

It is worth thinking about this relatively simple proof for a while, because the implications of the theorem are far-reaching. The theorem is general and non-linear. This means that, if a controlled robot is not passive, it is possible to construct an environment-maybe by a second controlled robot - that would be passive, but when connected to the original robot would result in an unstable system. This clearly gives a strong argument to create a passive behaviour for any robot that will potentially interact with an unknown environment, in order to ensure stable and safe behaviour. 


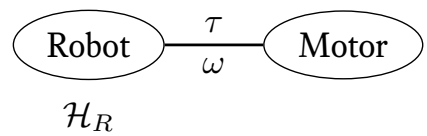

Figure 2.6 - A robot as a Port-Hamiltonian System connected to its actuator through a power port. The rate of change of its Hamiltonian $\mathcal{H}_{\mathrm{R}}$ is the supplied power, $\tau \cdot \omega$.

\section{$\S 2.2$ Energy and Distributed Architectures}

If a robotic system is seen as a Port-Hamiltonian System, then an actuator interacts with it through a port. In the case of an electric motor, the port variables are torque $\tau$ and rotational velocity $\omega$, Figure 2.6. Not regarding internal losses and interaction with an environment, the change of the robot's internal energy, $\dot{\mathcal{H}}_{\mathrm{R}}$, is the supplied power, $\tau \cdot \omega$.

\section{Measuring energy}

In order to measure the energy supplied to the robot, one could measure the electrical power going into the motor. However, an - often largepart of that energy is directly converted into heat, rather than mechanical energy. Instead, it is possible to directly and precisely measure the supplied mechanical energy $\Delta E$ between two times $t_{0}$ and $t_{1}(2.13)$.

Electrical motors are usually current-controlled, at least in an inner loop, meaning that their torque $\tau$ is precisely known if the motor constant $K_{m}$ is known (2.14). Moreover, most digital control systems use a zeroorder-hold $(\mathrm{ZOH})$ for their outputs during a sample period. Suppose that $t_{0}$ and $t_{1}$ are two consecutive instants in which the sampling of the position of the actuated joint takes place. At the same time the new set-point of a current, and therefore a torque, takes place and it will be held constant $(\mathrm{ZOH})$ until the next sampling instant. In this way, the current is kept constant over the integration period (2.15) and the energy injected by the actuator can be measured exactly (2.16). In this last equation, $q\left(t_{i}\right)$ is a position measurement of the motor at a sampled time instant. 


$$
\begin{array}{rlr}
\Delta E & =\int_{t_{0}}^{t_{1}} \tau(t) \cdot \omega(t) \mathrm{d} t & \text { supply rate } \\
& =\int_{t_{0}}^{t_{1}} K_{m} i(t) \cdot \omega(t) \mathrm{d} t & \text { motor constant } \\
& =K_{m} i\left(t_{0}\right) \int_{t_{0}}^{t_{1}} \omega(t) \mathrm{d} t & \mathrm{ZOH} \\
& =K_{m} i\left(t_{0}\right) \cdot\left(q\left(t_{1}\right)-q\left(t_{0}\right)\right) &
\end{array}
$$

It is important to realise that this is indeed the exact amount of injected energy, not an estimate. It can be measured if two conditions are met:

1. A zero-order hold is used for the current or motor torque.

2. A position sensor is collocated with the motor.

Note that there are no conditions on the sample time. This method of passive sampling was first introduced in [145]. Later, in Stramigioli et al. [146], the sampled energy was sent as an "energy packet" through discrete-time scattering between two haptic devices, ensuring a passive interconnection regardless of communication delays or losses.

Of course, if there is uncertainty in the measurement of $i$ or $q$, that uncertainty will carry to $\Delta E$. Specifically, in the case of angular encoders with a resolution of $\Delta q$, the maximum error of the measurement $\Delta \hat{E}$ is known:

$$
q\left(t_{i}\right) \in\left[\hat{q}\left(t_{i}\right)-\frac{\Delta q}{2}, \hat{q}\left(t_{i}\right)+\frac{\Delta q}{2}\right) \Rightarrow|\Delta \hat{E}-\Delta E|<K_{m} i\left(t_{0}\right) \Delta q .
$$

In this equation, $\hat{q}$ is the quantised measurement of $q$.

Example 2.4. Consider the system in Figure 2.7: a mass can be lifted by an electric motor, through a gearbox and pulley. The motor current is set by a PID controller. This system is simulated, where the setpoint is a cycloid (smooth step) from 0-0.5 $\mathrm{m}$ for the mass, executed between 1 and $3 \mathrm{~s}$. See Table 2.1 for all parameters of the simulation.

The energy measurement block $\hat{E}$ implements the result of (2.16) as follows, where $\hat{i}$ and $\hat{q}$ indicate the discrete-time variables, as labelled in Figure 2.7.

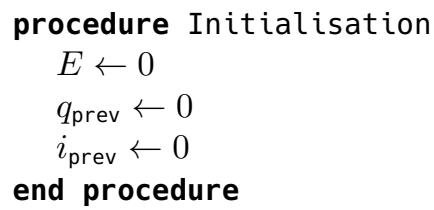




\begin{tabular}{rll}
\hline Parameter & Value & Description \\
\hline$K_{m}$ & $0.04 \mathrm{~N} \mathrm{~m} \mathrm{~A}^{-1}$ & Motor constant \\
$J_{m}$ & $1 \times 10^{-8} \mathrm{~kg} \mathrm{~m}^{2}$ & Motor inertia \\
$R$ & $4 \times 10^{-7} \mathrm{~N} \mathrm{~s} \mathrm{rad}^{-1}$ & Viscous motor friction \\
$n$ & 30 & Gear reduction ratio \\
$r_{p}$ & $0.02 \mathrm{~m}$ & Pulley radius \\
$M$ & $1 \mathrm{~kg}$ & Mass \\
$K_{\mathrm{P}}$ & $1.5 \times 10^{-4}$ & PID proportional gain \\
$\tau_{\mathrm{D}}$ & $0.4 \mathrm{~s}$ & PID derivative time constant \\
$\tau_{\mathrm{I}}$ & $5 \mathrm{~s}$ & PID integral time constant \\
\hline
\end{tabular}

Table 2.1 - Parameters used in the simulation of Figure 2.7.

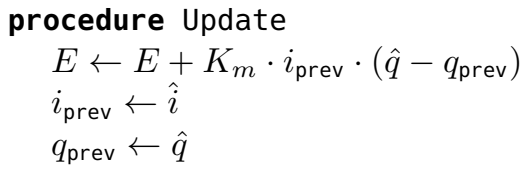

\section{end procedure}

The result of the simulation with various sample frequencies $f_{s}$ for the discrete-time part is shown in Figure 2.8. While the controller performance clearly deteriorates for very low sampling frequencies, the energy measurement is actually always exactly equal to true injected energy.

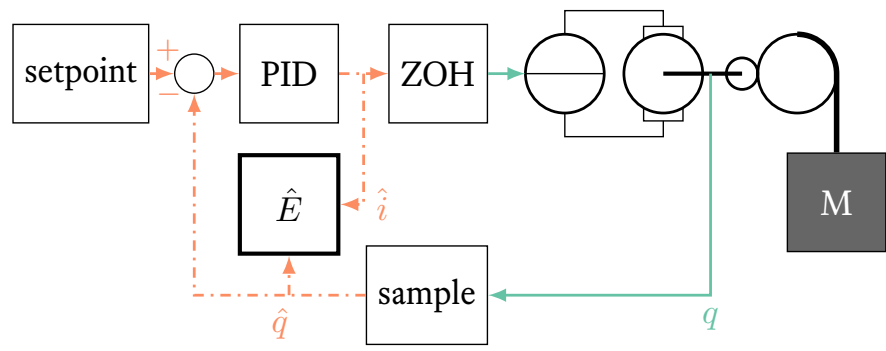

Figure 2.7 - A mass that can be lifted by a current-controlled motor. A discrete-time PID controller tries to follow a cycloid setpoint from $0-0.5 \mathrm{~m}$ between 1 and $3 \mathrm{~s}$. The energy injected by the motor is measured exactly by the block $\hat{E}$.

$\langle$ example end $\rangle$

Example 2.5. When the position measurement is quantised, for example by an encoder with limited counts per revolution (cpr), the energy bounds calculation from (2.17) can be implemented as follows: 


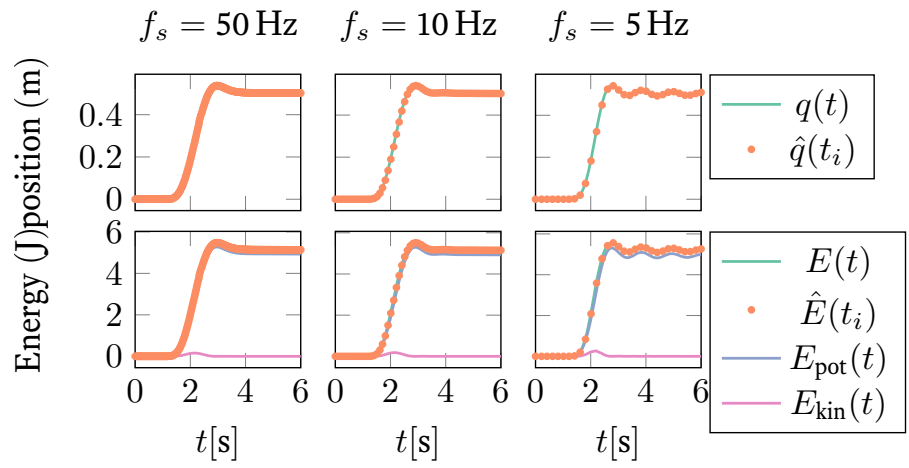

Figure 2.8 - Simulation of a PID-controlled mass that is lifted to $0.5 \mathrm{~m}$. The energy is measured exactly, according to (2.16). Most of the injected energy $E$ goes into potential energy of the mass $E_{\text {pot, }}$ but some is lost to friction. While moving, the mass has some kinetic energy $E_{\text {kin }}$. Note that for low sample frequency the controller loses performance, but the energy is still measured exactly.

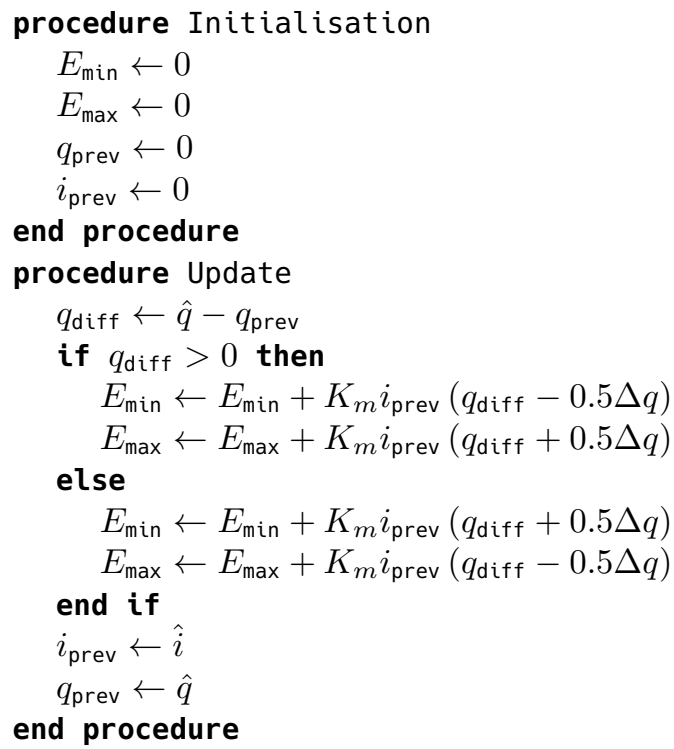

In the simulation, the position measurement is quantised by truncation:

$$
\hat{q}=q-(q \bmod \Delta q) ; \quad \Delta q=\frac{2 \pi}{\mathrm{cpr}} .
$$

The two simulations in Figure 2.9 show that the true injected energy always lies between the calculated bounds. Considering the second experiment, 
with a sample frequency of only $10 \mathrm{~Hz}$ and an encoder with only one count per revolution, this is remarkable indeed.

A potential problem with the method does become apparent from these figures: the bounds are widening as time passes, because theoretically the motors could always be jittering back and forth within a single encoder step in the worst possible way, continuously extracting energy from or injecting energy into the system. In practise, the encoder resolution will be much higher and this problem mitigated tremendously. Additionally, an external energy observer might be added to compensate for the drift of these bounds. However, the guarantee that the true energy lies strictly within the bounds would be lost. Alternatively, a worst case approach could be used in order to ensure that the energy injected in the system will be overestimated, as in [127].

$\langle$ example end $\rangle$

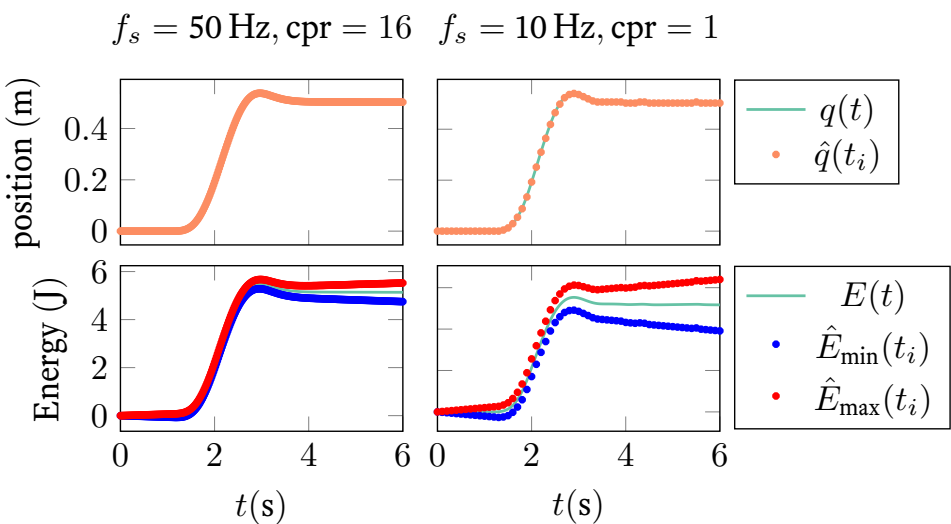

Figure 2.9 - Simulation of Figure 2.7, but now with a quantised motor position measurement. Using (2.17), exact bounds on $E$ are calculated. Note that even with only 1 count per revolution (cpr) and low sample frequency, the bounds remain quite close to the actual energy.

We have carried out experiments with a simple 1-DoF robot arm, measuring the energy according to (2.17). When the motor is controlled with a proportional position controller, it behaves as a virtual spring with stiffness $K_{\mathrm{P}}$ (the proportional gain). The "virtual energy" that is inside this virtual spring is given by

$$
E_{\mathrm{C}}=K_{\mathrm{P}} e^{2}
$$

where $e$ is the position error, or indeed the virtual spring state. This energy should match exactly with the energy that is extracted from the system, i.e. $E_{\mathrm{C}}=-\hat{E}$. As Figure 2.10 shows, when manually pushing 
the arm away from its setpoint, the internal controller energy is exactly equal to the measured energy.

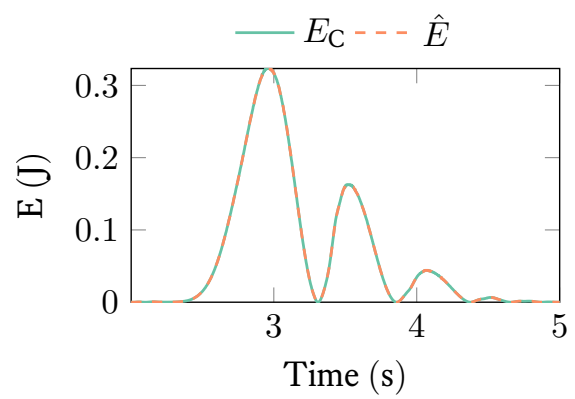

Figure 2.10 - Experiment with P-controller on a 1-DoF robot arm. The virtual energy present in the controller, $E_{\mathrm{C}}=K_{\mathrm{P}} e^{2}$, is exactly equal to the sampled energy $\hat{E}$.

\section{$\S 2.3$ Energy budgets}

From the stability-passivity criterion in Section 2.2.1: Passivity as a must, it is clear that in some cases - robots interacting with unknown environments - it is indispensable for stability to have a strictly passive control system. In other cases, it may still be advisable to limit the amount of energy that can be injected into the robot by the actuators: considering that damage and injury strongly correlate with energy transfer, limited energy greatly enhances the safety of a robotic system.

This can be done with energy observers that make use of knowledge of the system, for example by limiting the velocity or force of the motors, as in [149]. However, with the exact energy measurement scheme, it is possible to limit the energy that actually enters the system, which is a safety measure that is completely independent from the system model (and thus from modelling errors).

The controller can be given an energy budget that is put into a virtual energy tank. The first work introducing this concept can be found in [38]. The energy flowing through the actuators, measured using (2.16), also comes from this tank. In case the actuators extract energy from the system, for example when they slow down a mass, the energy of course flows back into the tank as well. When the tank is empty, the controller is no longer allowed to inject additional energy into the system: then the controller is guaranteed to be passive.

Since the power flowing into the robot is given by $F \cdot v$ (or $\tau \cdot \omega)$, preventing the injection of more energy is achieved by forcing either the force or the velocity to zero. (Note that a control action that would cause 
energy extraction can still be allowed.) See Figure 2.11 for a Finite State Machine implementation of the energy tank.

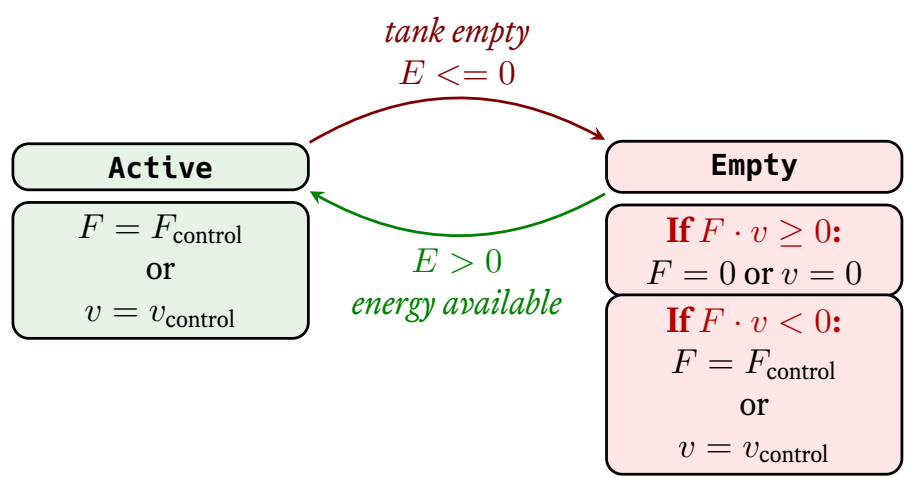

Figure 2.11 - A Finite State Machine implementation that, given the current energy tank level $E$, prevents further energy injection if the tank is empty.

Example 2.6. Consider the system depicted in Figure 2.12: a mass is lifted by a pulley, driven by an electric motor. The motor command is a constant torque resulting in a force on the mass greater than its weight, so the bucket is lifted. This action injects much (potential) energy into the system. By implementing the energy-sampling algorithm of (2.16) and the FSM of Figure 2.11, the amount of injected energy is limited: the actuator is given an energy budget of $5 \mathrm{~J}$; the mass of $1 \mathrm{~kg}$ is lifted until this energy has been used up, after which the mass is held steady.
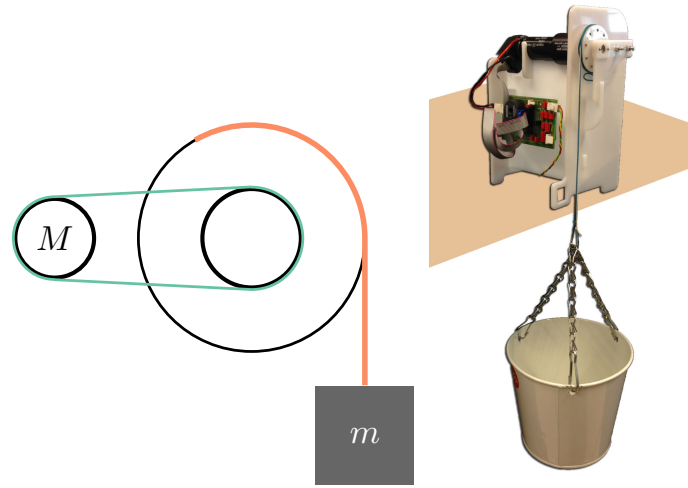

Figure 2.12 - A motor $M$ that lifts a mass $m$ by means of a pulley.

Around $t=6.5 \mathrm{~s}$, the mass is pulled down manually, which-acting 


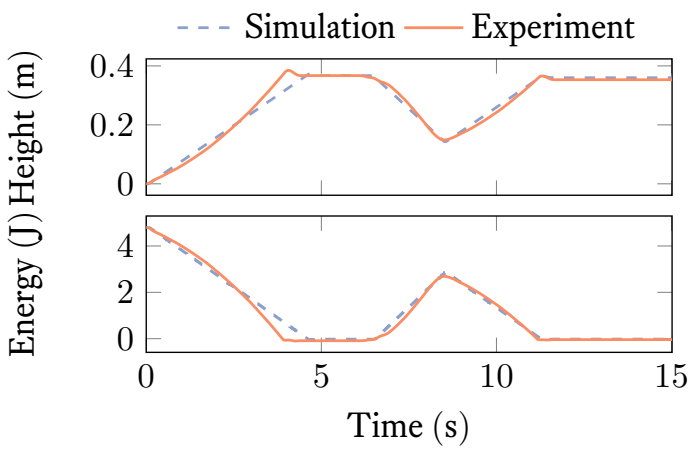

Figure 2.13 - Lift experiment and simulation: the mass is lifted by a constant torque, with the energy tank algorithms in place. Around $t=6.5 \mathrm{~s}$, the mass is pulled down manually.

against the holding torque-injects energy back into the virtual tank, allowing the controller to resume normal operation until the energy is used up again.

Note that the mass does not quite reach its expected height of $h=$ $5 \mathrm{~J} /\left(1 \mathrm{~kg} \cdot 9.8 \mathrm{~m} \mathrm{~s}^{-2}\right) \approx 0.5 \mathrm{~m}$ due to friction in the gearbox between motor and pulley. Dissipation can be a serious problem in passivity-based control, but as shown in Theorem 2.2.1, non-passive control may cause serious stability and safety problems.

$\langle$ example end $\rangle$

With the energy tank and an energy budget, the controller is guaranteed to be passive, regardless of the control algorithms. To see this, take the integral form of the dissipation inequality (2.7):

$$
S\left(x_{0}\right)+\int_{t_{0}}^{t_{1}} w(u(t), y(t)) \mathrm{d} t \geq S\left(x_{1}\right) .
$$

With $S\left(x_{0}\right)$ the initial energy in the tank and supply rate $w=\tau \cdot \omega$, it is clear that the energy left in the tank at time $t_{1}, S\left(x_{1}\right)$, is the initial energy $S\left(x_{0}\right)$ minus the injected energy, satisfying the (in)equality.

Hence, with a passive mechanical system driven by this passivated controller, the overall system is also guaranteed to be passive and thus stable (Theorem 2.2.1).

Appendix C contains a not-yet-published paper on the practical implementation of actuators with an energy budget. 


\section{§3 Control by interconnection}

In classical control theory, the inputs and outputs of the controller and plant are treated as signals. A sensor in the plant gives feedback to the controller $(y)$; the output of the controller is an input to the plant $(u)$ (Figure 2.14). The dimensions of $u$ and $y$ do not necessarily match in a Multi-Input, Multi-Output (MIMO) control system.

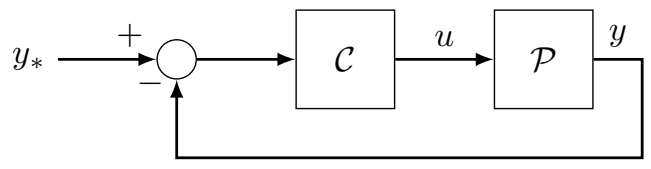

Figure 2.14 - A classical control system treats the inputs and outputs of plants and controller as signals.

This method of control does not lend itself well for energy-based robotics: if the sensors are not necessarily collocated with the actuators, it is impossible to measure or influence the exchanged energy as in Section 2.2.2: Energy and Distributed Architectures. Moreover, it is often hard to give a physical interpretation of a signal-based control law, which makes the controller itself sometimes difficult to understand.

From Figure 2.6 it is clear that in reality, all actuators in a robotic system actually exchange energy and physical quantities in a bi-directional way: in the case of electrical motors, electrical power is converted to mechanical power and exchanged with the system. This bi-directional flow of energy and physical variables can be extended to the control system: with this paradigm, the controller is no longer a signal-processor with separate inputs and outputs, but is a (Port Hamiltonian) system connected to the robot via power ports, as shown on the left in Figure 2.15.

In reality, the actuators sit between the controller and the plant. Or, drawing the boundary somewhere else, the actuators may be considered part of the plant, too, and the electrical circuit (current amplifiers) sit between controller algorithm and mechanical plant. In any case, the actuators are connected to a power source and have losses, so the drawing on the right in Figure 2.15 is more accurate. However, with proper considerations as described in the previous section, Section 2.2.2: Energy and Distributed Architectures, the actuator does become a power-continuous "transparent" connection between controller and plant.

With both the controller and plant described as a Port-Hamiltonian System, physical interpretation can be given to both of them. And, because the interconnection of two PHSs is again a PHS, this approach is inherently modular. ${ }^{3}$ Most importantly, because the connection between

${ }^{3}$ The Port-Controlled Hamiltonian Systems structure is not strictly necessary: it is the 


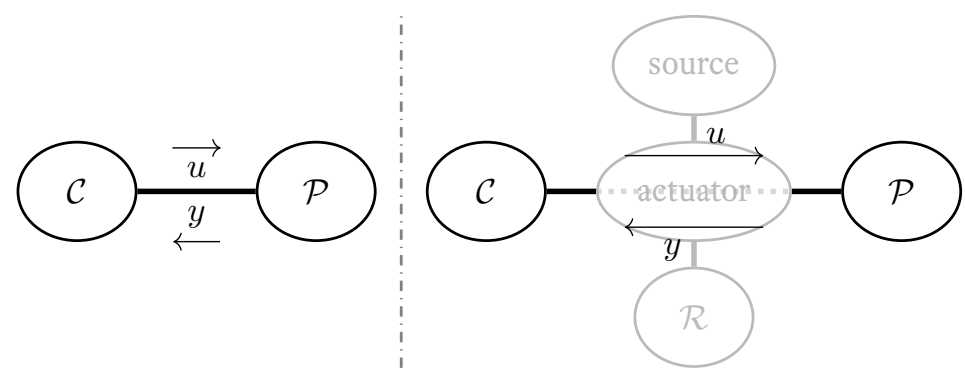

Figure 2.15 - The controller is a (Port-Hamiltonian) system connected to the robot via power ports (left). In real robotic systems, the actuators sit between the controller and mechanical system, but can be made "transparent" with the energy sampling method presented earlier.

controller and plant is power-continuous, there is direct control over the energy in the system. Passivity or energy-budgeted control are now inherent in the controller design. This approach of control is called control by interconnection; see [144], for likely the first real example in this context; and [94] and [95] for an in-depth mathematical treatment. In this section, we will consider the application to robotics.

\section{$\S 3.1$ Impedance control}

Impedance control was introduced by [65] as an approach to manipulation, after realising that force or position control is inadequate for real interaction tasks:

"Because of dynamic interaction [with the environment], the manipulator may no longer be treated ... as an isolated system. Strategies directed toward the control [of] position, velocity or force will be inadequate as they are insufficient to control the mechanical work exchanged between the manipulator and its environment."

In his three-part paper series, Hogan argues that most environments behave like an admittance-mass-like - so that the controller must behave as an impedance. Often, the controller (actuator) is directly connected to the robot's mass, an admittance, so even without regarding the environment, the controller should be an impedance. This means that a controller is defined by its port behaviour, by the relation between force and velocity. The impedance versus admittance is a "causality" argument, which regards the robot's velocity as an input to the controller

power-continuous interconnection that is vital; passivity properties can always be restored with the energy tank approach. 
and, consequently, the output of the controller is a force. It does not in principle say anything about how this relation should be implemented.

In the examples of Hogan, a second order behaviour has been used as an example of such a control strategy. By shaping the second order behaviour, the compliance, damping and the felt inertia-also called driving-point inertia - may be changed. Often, this specific implementation and choice has been considered in literature as "The impedance control approach," instead of the much more general causality and portbehaviour argument mentioned before. A characteristic example of this debate was published in [161] and the reaction by [167].

A simpler implementation of impedance control not trying to change the driving point inertia is compliance control. In this approach, a mechanical impedance can be a combination of a spring and a damper, so with $x$ the end-effector position and $v$ its velocity, the control force is determined by:

$$
F_{\text {impedance }}=-K \cdot\left(x-x_{0}\right)-b \cdot v,
$$

where $x_{0}$ is the end-effector position at zero potential energy and $K, b$ are the controller parameters: stiffness and damping coefficients, respectively. Since the two behaviours that are implemented - the spring and damper - are both physical systems, it is easy to describe (2.21) as a PortHamiltonian System, and recognise that the combination $\left(F_{\text {impedance }}, v\right)$ is a power port interconnection between the PHS of the controller and that of the physical robot, as depicted in Figure 2.16. The PHS equations of the controller are now:

$$
\begin{aligned}
\mathcal{H}(x) & =\frac{K}{2}\left(x-x_{0}\right)^{2} \\
\dot{x} & =(0) \frac{\partial \mathcal{H}}{\partial x}(x)+(1) \cdot v \\
F & =(1) \frac{\partial \mathcal{H}}{\partial x}(x)+b v .
\end{aligned}
$$

(The term $b v$ is a direct feed-through from input to output.) The powercontinuous interconnection is characterised by the Dirac structure or network structure

$$
F_{\text {robot }}=-F_{\text {control }}, \quad v_{\text {control }}=v_{\text {robot }} .
$$

The power-continuity of this interconnection can be shown by considering the power flowing through both ports:

$$
P_{\text {robot }}=F_{\text {robot }} v_{\text {robot }}=-F_{\text {control }} v_{\text {robot }}=-F_{\text {control }} v_{\text {control }}=-P_{\text {control }} \text {. }
$$


The sum of $P_{\text {robot }}$ and $P_{\text {control }}$ is zero and the network structure is indeed a proper Dirac structure. It is actually possible to expand the interconnection of this controller PHS with the robot as a mass into the same system as Figure 2.5, where the mass $\mathcal{I}$ is "physical" and the spring and friction are "virtual", implemented in the controller.

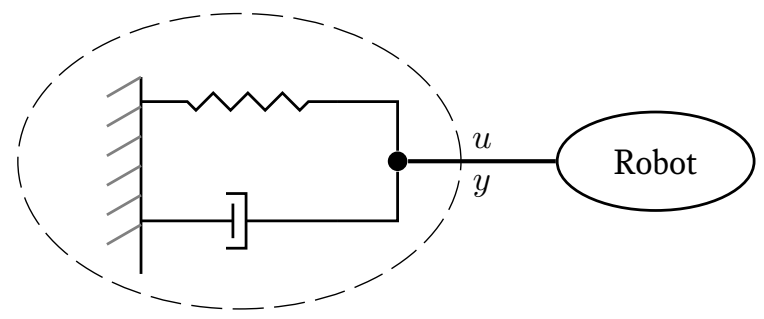

Figure 2.16 - Port-Hamiltonian interpretation of the impedance control strategy: the port variables $u$ and $y$ are the controller force and velocity, respectively.

A last important issue which should be considered is the fact that the interconnection is defined via port variables, which are force and velocities in the mechanical domain. By implementing the compliance, the position of the robot is used instead. A proper formulation to understand how to handle this issue is proposed in [144].

\section{Port behaviour and interaction}

It is important to realise a subtle but fundamental issue which is often not regarded in the literature. Force control and position control are not always well-posed problems, considering that the force or position of the end effector of te robot is a consequence of both the controller-which we can influence - and of the environment-which we cannot influence.

To clarify the issue: it is impossible to apply a desired force if no environment is touched, or to position an end-effector behind a rigid wall. On the other hand, it is always possible and well-defined to achieve the dynamics of (2.21) and controlling $K, x_{0}$ and $b$, independently of whether we move in free air or touch a very stiff wall. It therefore makes sense to control the behaviour $\left(K, x_{0}, b\right)$ : this is possible in all interaction situations, unlike when trying to control signals like force or position ([143]). This issue will be treated in more detail hereafter.

\section{Stability of impedance control}

An important note on stability and passivity with impedance control: as drawn and analysed here, the reference position $x_{0}$ is assumed to be constant. The virtual spring and damper are connected to a fixed world, 
the controller PHS is a passive physical system, hence the total system is guaranteed to be stable.

Often, however, $x_{0}$ is a time-dependent reference trajectory. In this case, although the controller seems to be a passive dynamical system, it is in fact in general not passive and therefore no guarantees can be given on stability. This is because the controller PHS has a second input $v_{0}$ $\left(\dot{x}_{0}\right)$, through which energy can be injected into the spring-damper and subsequently into the robot.

\section{Comparison to position and force control}

Position control Control strategies that aim for a position error $e \rightarrow 0$ as $t \rightarrow \infty$ are only meaningful for robotic manipulators that do not touch a (rigid) environment and are thus very different from impedancecontrolled robots. However, there is a large overlap as well: a PDcontroller is identical to a spring-damper impedance controller where $K_{\mathrm{P}}$ and $K_{\mathrm{D}}$ (the proportional and derivative gain) are equal to $K$ and $b$ of (2.21).

The distinction lies in the control objective: an impedance controller really aims to implement a certain stiffness and damping, whereas in position control the gains are usually maximised to reach a minimal position error. In the case of integrating action, the position controller loses all direct equivalence with impedance control. The equivalence could be regained by varying the rest position of the spring as derived below in (2.25), but as remarked before, varying $x_{0}$ generally injects energy, hence passivity is lost.

$$
\begin{array}{rlrl}
F_{\mathrm{PI}} & =K e+K_{\mathrm{I}} \int e \mathrm{~d} t & e=x_{s}-x \\
F_{\mathrm{imp}, \mathrm{K}} & =K\left(x_{0}-x\right) & & x_{0}:=x_{s}+\frac{K_{\mathrm{I}}}{K} \int\left(x_{s}-x\right) \mathrm{d} t \\
& =K\left(x_{s}+\frac{K_{\mathrm{I}}}{K} \int\left(x_{s}-x\right) \mathrm{d} t-x\right) & \\
& =K e+K_{\mathrm{I}} \int e \mathrm{~d} t &
\end{array}
$$

Force control In interaction tasks, one can also control the force applied to the environment. The main drawback is that force control is only possible if there is contact between the manipulator and the environment. In some cases there is an equivalence between force control and impedance control: if the manipulator does not move-for instance with an infinitely stiff environment - then $\dot{x}=0$ in (2.22) and $F$ is constant; [161]. However, there is again a clear distinction: impedance control 
determines the dynamic interaction behaviour of the manipulator and the observed equivalence only holds in some very specific cases; [167].

Impedance control In specific situations there can be an equivalence to some form of position control; and in other cases to some form of force control. However, impedance control is far more general in that it determines the port-behaviour at the interaction port between robot and environment. That behaviour is always independent of the specific environment and can be controlled independently from it.

Example 2.7. Implementing the spring-damper impedance control of Figure 2.16 in practice leads to two difficulties:

1. Real motors are usually equipped with position sensors, not velocity sensors, so implementing the damper requires some form of velocity observer.

2. The actuator limits may cause the actual control force $u$ to deviate from the desired force.

Both these issues can be solved in an elegant way by extending the controller with an extra (virtual) spring and mass, as shown in Figure 2.17 and introduced in $[139,96]$.

1. If the coupling spring is sufficiently stiff and the virtual mass sufficiently small, that is to say,

$$
k_{\text {coupling }} \gg k_{\text {control }} ; \quad \mathrm{m} \ll \mathrm{M} \text {, }
$$

then $v_{\mathrm{m}} \approx v_{\mathrm{M}}$. Because the virtual mass $\mathrm{m}$ is simulated, its velocity is known; and to calculate the control force $u$, only the displacement of $M$ needs to be known - which is measured by a position sensor.

2. The coupling spring is piecewise linear, flattening at the maximum actuator force $F_{\max }$ :

$$
F_{\text {coupling }}= \begin{cases}F_{\max } & k_{\text {coupling }} x_{\text {coupling }} \geq F_{\max } \\ -F_{\max } & k_{\text {coupling }} x_{\text {coupling }} \leq-F_{\max } \\ k_{\text {coupling }} x_{\text {coupling }} & \text { elsewhere }\end{cases}
$$

This way, the force calculated by the impedance controller can always be met by the actuator and the control system behaves as expected. 


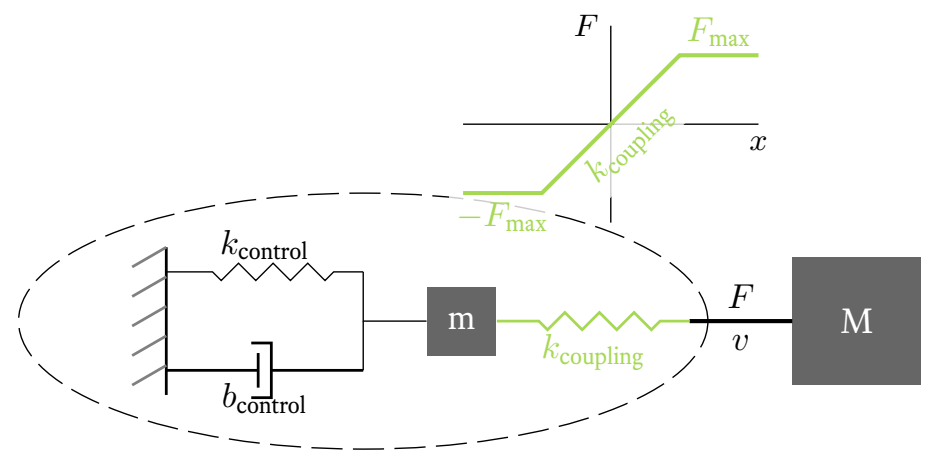

Figure 2.17 - Damping injection by extending the regular spring-damper impedance controller with an extra mass and (nonlinear) spring. They solve the problems of velocity observation and actuator saturation.

\section{$\S 3.2$ Energy shaping}

The impedance control law of (2.21) implements a spring pulling the endeffector to the virtual position $x_{0}$. Another way to look at this is that the energy function of the PHS of this same controller, (2.22), implements a potential energy function quadratic in $x$, with a minimum at $x_{0}$.

This idea, energy shaping, can be applied in a more general way. The method is well-known in the literature; here we look at it from a PortHamiltonian System point of view. Consider a robot mechanism described by

$$
M(q) \ddot{q}+C(q, \dot{q}) \dot{q}+\frac{\partial V(q)}{\partial q}=\tau,
$$

where $q$ are the joint positions and $\tau$ the applied joint torques; $M$ its inertia matrix; $C$ describes the Coriolis and centrifugal forces; and $V$ the potential energy.

In this fully-actuated collocated control system, it is possible to construct the control force $\tau$ from the derivative of some controller potential energy $V_{\mathrm{c}}$ :

$$
M(q) \ddot{q}+C(q, \dot{q}) \dot{q}+\frac{\partial V(q)}{\partial q}=-\frac{\partial V_{\mathrm{c}}(q)}{\partial q}
$$

such that the closed-loop dynamics become

$$
M(q) \ddot{q}+C(q, \dot{q}) \dot{q}+\frac{\partial\left(V(q)+V_{\mathrm{c}}(q)\right)}{\partial q}=0 .
$$

In other words, the robot behaves as a mechanism with a new potential energy $V+V_{\text {c }}$. If $V(q)$ is known, it can even be fully compensated by 
setting $V_{\mathrm{c}}=-V+V_{\mathrm{c}}^{\prime}$ and the robot given an arbitrary desired potential energy $V_{\mathrm{c}}^{\prime}$ with its minimum at a desired $q_{0}$.

From a Port-Hamiltonian System point of view, the controller is interconnected to the robot's power port $(\dot{q}, \tau)$. The controller's PHSequations become:

$$
\begin{aligned}
\mathcal{H}(x) & =-V(x)+V_{\mathrm{c}}^{\prime}(x) \\
\dot{x} & =u \\
y & =\frac{\partial \mathcal{H}}{\partial x}(x)
\end{aligned}
$$

with the Dirac structure $(u=\dot{q}, \tau=-y)$. These interconnected systems are again a PHS with total energy

$$
\mathcal{H}_{\text {tot }}(q, x)=(V(q)-V(x))+V_{\mathrm{c}}(x)+\dot{q}^{\top} M(q) \dot{q} .
$$

Due to the interconnection, $x=q$ and so $V_{\mathrm{c}}$ can be designed such that its minimum is at the desired equilibrium $q_{0}$.

If there is no or insufficient damping present in the robot, this may be added, e.g. by a damping matrix $B(q) \geq 0$ adding a damping force $B(q) \dot{q}$ to $\tau$ of (2.28). It is implemented as a direct feed-through term like in (2.22), by adding a term $B(x) u$ to the output expression of (2.31). This damping injection dissipates (free) energy, reducing $\mathcal{H}(x)$ to its minimum of $\mathcal{H}_{\text {tot }}\left(q_{0}, q_{0}\right)$ sooner, as shown by the dissipation relation of (2.4):

$$
\dot{\mathcal{H}}_{\text {tot }}(q, x)=-\dot{q}^{\top} B(x) \dot{q} .
$$

Remarks on energy shaping

- In the example above, we have made $x$ equal to $q$ by setting $g(x)$, the input/state/output mapping of (2.3), equal to 1. Instead, $g(x)$ can be used for a coordinate transformation to shape the potential energy in some other coordinate system, e.g. Cartesian endeffector coordinates.

- In the case of underactuated robots, the potential energy may not be fully reshaped. In that case, $V(q)-V(x)$ in (2.32) does not disappear-still, the system converges to the minimum of $\mathcal{H}_{\text {tot }}$. Closely related to this is the issue of state detectability: if some state variables of the plant are undetectable, it is impossible to shape the energy related to those coordinates.

- In general, there might not be a direct one-to-one connection between the full plant state $x$ and the controller state $q$ as assumed in the example. A method to obtain a connection between $x$ and $q$ is to generate Casimir functions for the connection ([144]). 
Casimirs are conserved quantities of $q$ and $x$ that can be used in Lyapunov candidates to find a stabilising control law. We refer to [26] and [123] for more on this topic.

- Energy shaping is a form of passivity-based-control: the controller is a passive Port-Hamiltonian System, so it can inject only a finite amount of energy, namely $\mathcal{H}(x(0))-\min \mathcal{H}(x)$. This means it is impossible to steer the system to a state in which it dissipates energy, e.g. to a constant nonzero velocity with friction loss. In the presence of dissipation - that is to say, in all relevant practical cases - it is therefore only possible to reach a state $(q, \dot{q})=\left(q_{0}, 0\right)$. In position control, this is exactly the goal, so this dissipation obstacle is of no concern. However, it should be noted that this energy shaping is actually only potential energy shaping. If some kind of (periodic) motion is the control objective, also the kinetic energy must be controlled, which is addressed in the next section.

\section{§3.3 Energy routing}

The concept of controlling the energy of a robotic system can be taken further. Before, the (potential) energy of the controller was shaped, thereby shaping the energy of the interconnected system. In this section, we will investigate how to directly influence the power flows between the controller and system(s).

By energy routing we refer to all control methods that do not influence the energy content of the system. That is, the total Hamiltonian of the interconnected system is constant; the control algorithms only direct the energy flow. The energy flows are determined by the interconnection of the systems - e.g. of the controller and robot-so energy routing takes place in the interconnection structure: by modulating the Dirac structure or the internal interconnection $J$, whilst keeping their skew-symmetry intact.

In this section, we will give various examples of energy routing. For further applications, we refer to existing work in $[38,119,48,39]$.

\section{Modulated buffer}

First, we introduce the concept of a modulated buffer, which is a passive controller structure that can be regarded as a force actuator with limited energy budget. The structure of the modulated buffer is drawn in Figure 2.18: the input-state-output $g$ of the Port-Hamiltonian System is modulated, which is equivalent to a modulated transformer element connected to a buffer, also shown in the figure.

Note that the output $y$ of this modulated buffer will form the input to 
the system it is connected to; the buffer's input $u$ is the system's output.

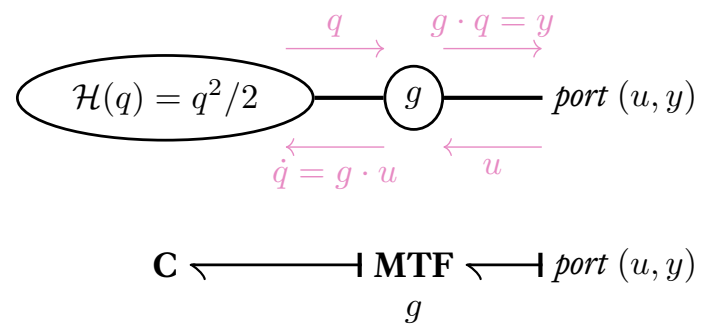

Figure 2.18 - Buffer with modulated input-state-output map (top); in bond graph expression (bottom) a $\mathbf{C}$-type storage with modulated transformer MTF.

Theorem 2.3.1. Consider a Port-Hamiltonian System with Hamiltonian $\mathcal{H}(q)=q^{2} / 2$ and scalar state-port map $g$ as drawn in Figure 2.18. $g$ is a pure modulation input and can be modulated such that the output of this PHS is an arbitrary force. The Port-Hamiltonian System is passive and the energy it can inject is bounded by $\mathcal{H}\left(q_{0}\right)$.

Proof. The PHS-formulation of this controller system is given by

$$
\begin{aligned}
\mathcal{H}(q) & =\frac{q^{2}}{2} \\
\dot{q} & =g \cdot u \\
y & =g \cdot q .
\end{aligned}
$$

With modulation

$$
g=\frac{F}{q}
$$

the output $y$ of the controller is

$$
y=g \cdot q=F,
$$

an arbitrary force, as long as $q \neq 0$. The energy change due to $g$ is $\partial \mathcal{H} / \partial g=0$, so $g$ is a purely modulating input.

Any energy injected into a system that is interconnected to the controller by

$$
u_{\text {system }}=y ; \quad u=-y_{\text {system }}
$$

is extracted from the Hamiltonian of the controller:

$$
P_{\text {system }}=u_{\text {system }} \cdot y_{\text {system }}=-y u=-\frac{\mathrm{d} \mathcal{H}}{\mathrm{d} t} .
$$


When $q=0$, the Hamiltonian becomes o too and the controller can no longer deliver a force that would decrease $\mathcal{H}$, i.e. the controller can no longer inject energy into the interconnected system. Therefore, the maximum energy this PHS-controller can inject is given by $\mathcal{H}\left(q_{0}\right)$, the initial energy in the controller.

This modulated-buffer approach may seem like a simple but useless trick: any control output is possible, as long as there is enough energy inside the buffer. If the buffer is empty, the controller stops working. However, this approach allows to turn any control algorithm into a passive controller, giving important dissipativity and stability guarantees. Similar to the energy-tank approach, the integral dissipation inequality of (2.7) is satisfied with $S\left(x_{0}\right)$ equal to $\mathcal{H}\left(q_{0}\right)$ and $w=y^{\top} u$. Any nonlinear feedback problem can be easily formulated with this approach, where the result is guaranteed to be passive. In [142], this method is used to create globally attractive, energy conservative limit cycle oscillations.

\section{Energy level control}

Considering the importance of energy in physical systems, sometimes it may be desirable to control the actual energy level in a robotic system, rather than a velocity, force or position. Consider for example a system that must execute a periodic motion along its natural dynamics, e.g. an oscillation: the energy level directly determines the amplitude and velocity of the system. Control of the energy present in the system is a robust and coordinate-independent way of keeping the motion on this specific (geodesic) path, with a certain desired amplitude.

Example 2.8. Consider the simple mass-spring system of Figure 2.19. A controller interacts with the system through a force/velocity port and its goal will be to maintain a certain amplitude of oscillation $x_{\mathrm{s}}$.

The PHS equations for the system are familiar:

$$
\begin{aligned}
\mathcal{H}(x) & =\frac{p^{2}}{2 \mathrm{~m}}+\frac{\mathrm{k}}{2} x_{\mathrm{m}}^{2} \\
\left(\begin{array}{c}
\dot{p} \\
\dot{x}_{\mathrm{m}}
\end{array}\right) & =\left[\begin{array}{cc}
0 & -1 \\
1 & 0
\end{array}\right] \frac{\partial \mathcal{H}}{\partial x}(x)+\left(\begin{array}{l}
1 \\
0
\end{array}\right) F_{\text {control }} \\
v & =\left(\begin{array}{ll}
1 & 0
\end{array}\right) \frac{\partial \mathcal{H}}{\partial x}(x),
\end{aligned}
$$

where the state vector $x$ should not be confused with $x_{\mathrm{m}}$, the mass position. 

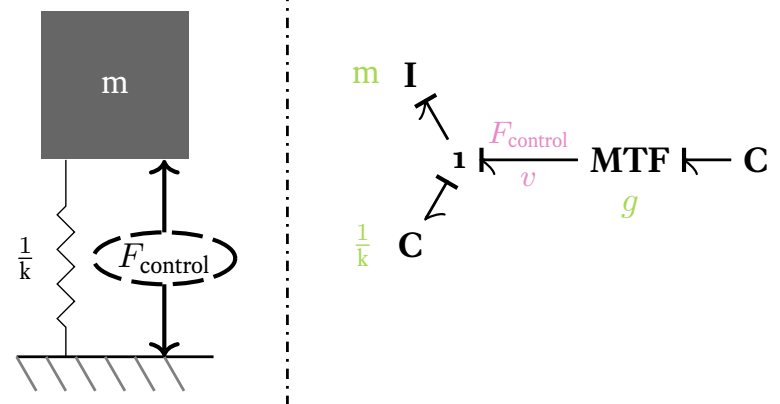

Figure 2.19 - Mass-spring system with force controller, aiming to keep the energy level constant. Left: Ideal Physical Model representation; right: bond graph representation.

The suggestion for a passive controller structure is the following:

$$
\begin{aligned}
\mathcal{H}_{c}(q) & =\frac{q^{2}}{2 \mathrm{C}} \\
\dot{q} & =g \cdot u \\
y & =g \cdot \frac{q}{\mathrm{C}},
\end{aligned}
$$

which is the modulated storage element of Theorem 2.3.1, but now with a capacity C. In Figure 2.19, the controller is shown as a bond graph representation of the storage element $C$ and a modulating transformer as the port-state map $g$. (While the bond graph formulation is not necessary for Port-Hamiltonian System theory, this example shows how well the two relate, since bond graphs are an explicit graphical representation of the various storage elements, ports and Dirac structures. We shall address bond graphs in this work no further, but refer to [98] and [17].) With the interconnection $u=-v, F_{\text {control }}=y$ the power injected by the controller, $P_{\mathrm{c}}$, can be calculated (2.41). However, $v$ is known - it is the input to the controller-and hence $P_{\mathrm{c}}$ can be controlled, by choosing $g$ as follows:

$$
P_{\mathrm{c}}=y v=\left(\frac{g q}{\mathrm{C}}\right) v \Rightarrow g:=\left(\frac{\mathrm{C}}{q v}\right) P_{\mathrm{c}} .
$$

Finally, by choosing $P_{\mathrm{c}}$ depending on the "energy error" $\tilde{E}$, the controller will inject or extract energy as needed:

$$
P_{c}=-\frac{\lambda}{2} \tilde{E} ; \quad \tilde{E}=\mathcal{H}(x)-\frac{\mathrm{k}}{2} x_{\mathrm{s}}^{2} .
$$

$\mathcal{H}(x)$ can be calculated from the measured $v ; \lambda$ is a free control parameter that determines the rate of convergence. 
This control law regulates the energy level of the system with exponential convergence, resulting in the desired oscillation amplitude of $x_{\mathrm{s}}$. The stability and convergence proof is given in Appendix B.

$\langle$ example end $\rangle$

\section{Irreversible transduction}

The dissipation matrix $R$ in Port-Hamiltonian Systems (2.3) is usually associated with energy loss. From a mathematical point of view, this is correct: the Hamiltonian decreases with the energy dissipated through $R$. Physically, however, energy is not lost; instead, dissipation means (irreversible) transduction to an entropy flow: it is converted to heat. A model for this irreversible transduction is a resistive element with two ports, the "RS" model: one mechanical with port variables $(F, v)$ and one thermodynamical with port variables $(T, \mathrm{~d} S)$, where $T$ is the absolute temperature and $\mathrm{d} S$ the entropy flow. The constitutive relations are as follows:

$$
F=-\mathrm{R} v ; \quad \mathrm{d} S=\frac{\mathrm{R} v^{2}}{T} .
$$

It is easy to check that the mechanical power $F v=-\mathrm{R} v^{2}$ is always negative and equal to the power flowing out of the thermodynamical port.

The relevance for energy-based control is that this is a power-continuous element that allows energy to flow only in one direction. The ports do not necessarily have to be mechanical and thermodynamical: the portbehaviour can be used inside a controller to direct (kinetic) energy from one element to another.

Example 2.9. Consider two masses interconnected by a controller that behaves as an RS-like irreversible transduction element. Because the masses require a force input, the "thermodynamic" port relation of $(2.43)$ has to be rearranged to output a force:

$$
F_{1}=-\mathrm{R} v_{1} ; \quad F_{2}=\frac{\mathrm{R} v_{1}^{2}}{v_{2}},
$$

which is still a power-continuous interconnection. The closed-loop equations are:

$$
\begin{aligned}
\mathcal{H}(x) & =\frac{p_{1}^{2}}{2 \mathrm{~m}_{1}}+\frac{p_{2}^{2}}{2 \mathrm{~m}_{2}} \\
\dot{x} & =\left(\begin{array}{cc}
0 & -\mathrm{R} \frac{v_{1}}{v_{2}} \\
\mathrm{R} \frac{v_{1}}{v_{2}} & 0
\end{array}\right) \frac{\partial \mathcal{H}}{\partial x}(x),
\end{aligned}
$$

with the two masses' momenta as the state vector. From (2.44), we would usually find " $-\mathrm{R}$ " in the upper-left position of the matrix in (2.46), but the 
upper-right term gives the same result:

$$
-\mathrm{R} \frac{v_{1}}{v_{2}} \frac{\partial \mathcal{H}}{\partial p_{2}}=-\mathrm{R} \frac{v_{1}}{v_{2}} \frac{p_{2}}{\mathrm{~m}_{2}}=-\mathrm{R} \frac{v_{1}}{v_{2}} v_{2}=-\mathrm{R} v_{1} .
$$

Now, however, the matrix is skew-symmetric and thus the system is losslessbecause the damping energy is not dissipated, but directed to the other mass.

Figure 2.20 shows a simulation of this example, with $\mathrm{m}_{1}=\mathrm{m}_{2}=1 \mathrm{~kg}$, $\mathrm{R}=0.5 \mathrm{~N} \mathrm{~s} \mathrm{~m}^{-1}$ and $x(0)=(10)^{\top} \mathrm{kg} \mathrm{m} \mathrm{s}^{-1}$. Mass 1 is slowed down by the friction of $\mathrm{R}$, but the dissipated energy is injected into mass 2 . The total energy remains constant.

$\langle$ example end $\rangle$

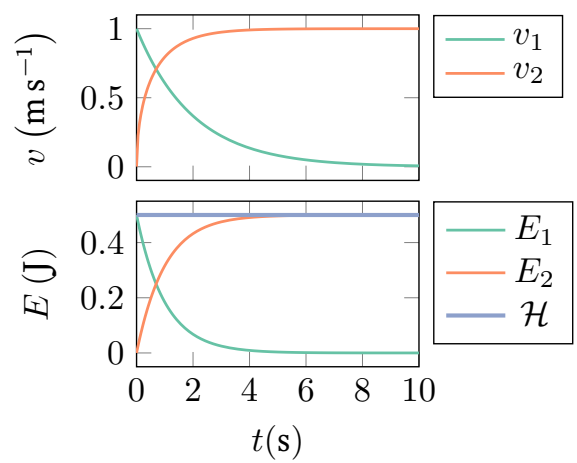

Figure 2.20 - Simulation of two masses interconnected by a powercontinuous controller that creates irreversible transduction from mass 1 to mass 2 . For mass 1 , the controller behaves as a friction, but all "dissipated" energy is diverted to mass 2. ( $E_{i}$ is the kinetic energy of mass $i$.)

\section{Kinetic energy routing}

The concept of irreversible transduction can be generalised further, to achieve arbitrary energy routing in the controller. A gyrator is a powercontinuous Dirac structure that connects two Port-Hamiltonian Systems as follows:

$$
\left(\begin{array}{l}
u_{1} \\
u_{2}
\end{array}\right)=\left[\begin{array}{cc}
0 & r \\
-r & 0
\end{array}\right]\left(\begin{array}{l}
y_{1} \\
y_{2}
\end{array}\right),
$$

with $r$ the gyration ratio. It is easy to verify that the connection is powercontinuous, but more importantly, the power flow through the interconnection is modulated by $r$.

Theorem 2.3.2. Given two Port-Hamiltonian Systems $\Sigma_{1}$ and $\Sigma_{2}$ with power ports $\left(u_{1}, y_{1}\right)$ and $\left(u_{2}, y_{2}\right)$ that are interconnected by a gyrator with 
gyration ratio $r$. If the gyrator is modulated according to

$$
r=\frac{1}{y_{2} y_{1}} \cdot r_{P}
$$

then the energy flow from $\Sigma_{2}$ to $\Sigma_{1}$ is equal to $r_{P}$, as long as $y_{1} \neq 0$ and $y_{2} \neq 0$. This interconnection is power-continuous and $r_{P}$ is a pure modulation to passively direct the energy flow between the two systems.

Proof. With the gyrating Dirac structure behaving according to (2.48), the power flows into $\Sigma_{1}$ and $\Sigma_{2}, P_{1}$ and $P_{2}$, are:

$$
P_{1}=y_{1} u_{1}=y_{1} r y_{2} ; \quad P_{2}=y_{2} u_{2}=-y_{2} r y_{1} \text {. }
$$

$P_{1}=-P_{2}$, so the interconnection is power-continuous. With the gyration ratio given by (2.49), $P_{1}$ is:

$$
P_{1}=y_{1} u_{1}=y_{1} r y_{2}=y_{1} \frac{1}{y_{2} y_{1}} r_{P} y_{2}=r_{P}
$$

Corollary 2.3.3. If the gyration ratio is instead set according to (2.52), the ratio is always well-defined-even if $y_{1}=0$ or $y_{2}=0$. However, for $y_{1}=0$ or $y_{2}=0$, still no energy transfer takes place; and while the direction of power flow is still controlled by $r_{P}$, the rate of energy exchange depends on $y_{1}$ and $y_{2}(2.53)$.

$$
\begin{gathered}
r=y_{1} y_{2} r_{P} \\
P_{1}=y_{1} u_{1}=y_{1} r y_{2}=y_{1}\left(y_{1} y_{2}\right) r_{P} y_{2}=\left(y_{1}^{2} y_{2}^{2}\right) r_{P}
\end{gathered}
$$

In practice, the condition that $y_{1} \neq 0$ and $y_{2} \neq 0$ is rather restrictive and the alternative gyration ratio does not allow output-independent control over the rate of energy exchange. However, as is shown in the following example, there is a way to get around the restriction, with little deviation between $r_{P}$ and the actual energy flow.

Example 2.10. Consider a simple example of two masses connected to a control system that implements the energy routing of Theorem 2.3.2, illustrated in Figure 2.21. The inputs $u_{i}$ represent a force; the corresponding outputs $y_{i}$ the masses' velocities.

In order to allow situations where $y_{1}=v_{1}=0$ and $y_{2}=v_{2}=0$, the gyrator ratio of (2.49) is adapted to:

$$
r=\frac{y_{1} y_{2}}{\left(y_{2}^{2}+\epsilon\right)\left(y_{1}^{2}+\epsilon\right)} r_{P}
$$




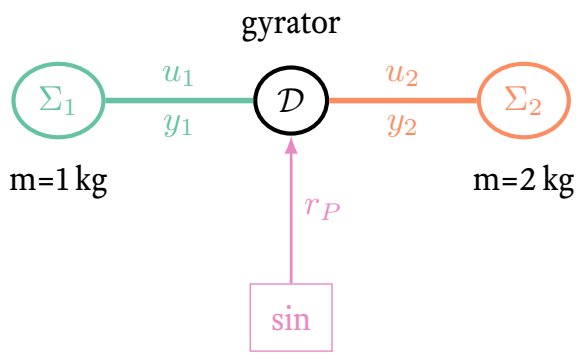

Figure 2.21 - Two masses connected by a gyrating controller that can route kinetic energy. The "s in" block generates a sinusoidal energy flow setpoint.

where $\epsilon>0$ is some small number. For large values of $y_{1}$ and $y_{2}$-that is, $y_{i}^{2} \gg \epsilon$-the gyration ratio will be approximately equal to (2.49). When $y_{i}=0$, it remains well-defined.

The system of Figure 2.21 is simulated with an initial velocity of $v_{1}=$ $1.1 \mathrm{~m} \mathrm{~s}^{-1}$ and $r_{P}$ is a sine wave of amplitude $0.2 \mathrm{~W}$ and period $10 \mathrm{~s}$. $\epsilon$ is set to 0.001 . Figure 2.22 shows the results: except for when $v_{1} \approx 0$ or $v_{2} \approx 0$, the actual energy flow to $\Sigma_{1}$ is equal to the setpoint $r_{P}$. The energy plot in the middle shows that the complete system is power-continuous, as was expected. $\langle$ example end $\rangle$

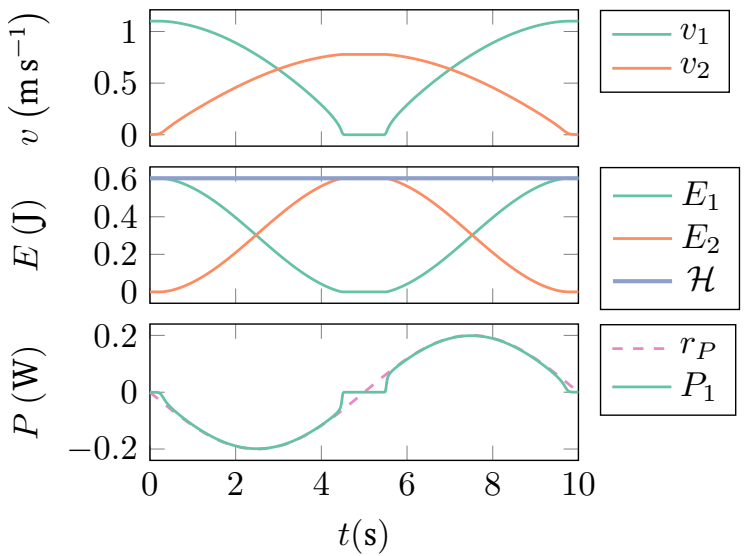

Figure 2.22 - Implementation of the kinetic energy router of example Example 2.10. Because of the adapted gyration ratio (2.54), when $v_{1}$ or $v_{2}$ gets close to 0 , the actual power flow $P_{1}$ deviates from $r_{P}$.

The previous example considered two simple systems $\Sigma_{1}$ and $\Sigma_{2}$. 
However, the concept of energy routing can be applied to much more complex systems. For example, the two ports do not have to be the velocity of two separate masses. It can also be the two velocity components of a mass moving in 2-D, or even those velocities in a transformed coordinate system, such that $v_{1}$ corresponds to the mass's velocity in a desired direction and $v_{2}$ the velocity perpendicular to that. By modulating $r_{P}$, all kinetic energy ${ }^{4}$ can be routed from the "unwanted" direction to the desired direction, thereby steering the mass along a certain trajectorywithout using external energy. In [38], this method is used to create passive limit cycles.

In [47], the gyrating controller is connected to the stiffness-changing ports of two springs $\left(\dot{K}, x^{2} / 2\right)$, adapting their stiffness in a powercontinuous way to achieve synchronisation between two oscillators by routing energy between the springs.

${ }^{4}$ Full energy transfer does require the systems, or at least the "source" system, to be zero-state-detectable. 


\section{$\S 4$ Control by physical interconnection}

In control by interconnection, the control algorithm has the dynamics of a Port-Hamiltonian System that could represent a physical system, e.g. the virtual springs and masses of Example 2.7. At the same time, the physical system can be represented as a PHS. The interconnection of the controller and system is of course again a Port-Hamiltonian System, which raises the question: what distinguishes the controller from the physical system? The answer is: conceptually and mathematically, nothing at all.

This means that the boundary between system and controller is a fluid, shifting boundary that is frozen only at the moment of implementation, when the interface between computer and physical system is decided. And even then, one might argue that part of the control effort is carried out by the system dynamics, or part of the system dynamics are simulated in the controller. In the previous section, we have studied control by interconnection, where the controller was represented by a Port-Hamiltonian System, which gives a physical interpretation to the control algorithms. In this section, we shall consider the physical system's PHS-representation in the light of the control objectives. We call this "Control by physical interconnection," where desired behaviour previously achieved by the controller is now achieved by the physical system itself.

\section{§4.1 Physical compliance}

Electric motors have many benefits when used in robotic systems: high availability in a broad range, easy electrical interconnection to the control system, integrated position encoders, a large selection of gearboxes, precise torque control, easy integration in rotational joints, et cetera. However, there is also a major drawback: electric motors are very efficient in a high speed/ low torque region, whereas many robotic systems usually operate in low speed/high torque regions.

An electric motor's efficiency is mostly determined by its motor constant $K_{\mathrm{m}}$ and its electrical resistance $R_{\mathrm{e}}$, for a given required torque output. In low speed regions, mechanical losses are negligible and the electrical loss is computed as:

$$
P_{\mathrm{e}, \mathrm{loss}}=i^{2} R_{\mathrm{e}}=\left(\frac{\tau}{K_{\mathrm{m}}}\right)^{2} R_{\mathrm{e}}=\frac{\tau^{2}}{S} .
$$

$S$ is the "motor steepness" and defined as $K_{\mathrm{m}}^{2} / R_{\mathrm{e}}$. Clearly, a higher motor steepness results in lower power loss. In motor fabrication, there is a tradeoff between size, weight and motor steepness: to increase $S$, the 
resistance must be low (thick wires), or the motor constant high (long wires, large motor diameter, high magnetic fields). For research on the analysis and improvement of electric motor efficiency, we refer to [130] and [37].

In practice, the motor with lowest losses may be picked, but there is still no way around the fact that for zero speed and nonzero torquewhich is the desired equilibrium in e.g. position control-the motor efficiency becomes zero:

$$
\eta_{\text {motor }}=\frac{P_{\text {mechanical }}}{P_{\text {loss }}}=\frac{\omega \tau}{P_{\mathrm{e}}} \underset{\omega=0, \tau \neq 0}{=} \frac{0}{\tau^{2} / S} .
$$

\section{Physical storage elements}

Much of the work that electric motors do in robotic applications is conservative, for example when moving a robot arm up and down in a gravity field. Indeed, if for stability or safety reasons the controller is strictly passive, as argued in Section 2.2.1: Passivity as a must, the net mechanical work of the motor is o. Therefore, much if not all of the work that the motor does could be done by a passive physical storage element: by a spring or by an inertia.

A flywheel stores kinetic energy, determined by its state, the momentum $p$ :

$$
E_{\text {flywheel }}(p)=\frac{p^{2}}{2 J},
$$

which is in value equal to the kinetic co-energy $E^{*}: 5$

$$
E_{\text {flywheel }}^{*}(\omega)=\frac{J \omega^{2}}{2}
$$

$J$ is the flywheel's moment of inertia. While flywheels are used in automotive industry ([84]), for example to make stops and starts of buses more efficient, they are not so suitable for robotics applications. This is because they store and release their energy typically at high speeds, which is exactly the region in which electric motors are quite efficient, and in which robotic systems typically do not operate. ${ }^{6}$

Springs, on the other hand, store potential energy, determined by the state $q$ :

$$
E_{\text {spring }}(q)=\frac{K q^{2}}{2}
$$

\footnotetext{
${ }^{5}$ The co-energy is a function of $\omega$, the port variable that is a thermo-dynamical intensive variable, rather than a function of (the extensive variable) $p$.

${ }^{6}$ As electrical energy storage, they are very suitable: through the gyrating motor/dynamo, the flywheel behaves as a capacitor-like storage element as seen from the electrical side.
} 
or potential co-energy

$$
E_{\text {spring }}^{*}(F)=\frac{F^{2}}{2 K},
$$

where $K$ is the spring stiffness. Because the spring can store and release energy at low or even zero velocity, it is very suitable for application in robotics, where it can assist electric motors in regions where the motor efficiency is very low. Furthermore, this energy is stored in a physical deformation in the mechanical domain, at zero velocity. Therefore, the energy is not lost to friction over time, as may be the case in a flywheel.

\section{Parallel spring configuration}

When the spring is connected in parallel to the motor, their torques are added:

$$
\tau_{\text {tot }}=\tau_{\text {motor }}+\tau_{\text {spring }}=\tau_{\text {motor }}+K\left(q-q_{0}\right) .
$$

The spring is characterised by two parameters: the spring stiffness $K$ and its rest length $q_{0}$. The spring can be dimensioned to supply quasi-static torque, for example for passive gravity compensation. In that case, a low $K$ combined with high $q_{0}$ leads to a nearly constant output torque, greatly lowering the static torque on the motor and thereby reducing electrical energy loss. In more dynamic applications, especially when the motion executed by the robot is periodic, an optimal spring can even deliver a large part of the required torque during the trajectory. In [68] and [163], parallel stiffness is applied in energy-efficient hopping robots. The use of balancing springs has been also extensively and elegantly treated in [63].

Example 2.11. Consider the simple 2-DoF robot arm in Figure 2.23, consisting of two rotational joints with joint angle $q_{i}$ and joint torque $\tau_{i}$. Its end-effector follows an elliptical trajectory according to $(2.62)$ in the $(x, y)$ plane, so there is no gravity interaction. The system parameters can be found in Table 2.2.

$$
p_{\text {setpoint }}=\left(\begin{array}{c}
0.5+0.8 \cos (\pi t) \\
1.2+0.3 \sin (\pi t)
\end{array}\right) \mathrm{m}
$$

The controller used in this example is a virtual prismatic spring and damper between the end-effector and the setpoint, with parameters according to Table 2.2:

$$
\left(\begin{array}{c}
\tau_{1} \\
\tau_{2}
\end{array}\right)=J^{\top}(q) \cdot\left(K\left(p_{\text {setpoint }}-p_{\text {ee }}\right)+D\left(\dot{p}_{\text {setpoint }}-\dot{p}_{\text {ee }}\right)\right) .
$$

$J(q)$ is the Jacobian map from joint velocity $\dot{q}$ to end-effector velocity $\dot{p}_{\text {ee, }}$ and so $J^{\top}(q)$ is the dual map from end-effector force to joint torque. The 


\begin{tabular}{rccl}
\hline Parameter & \multicolumn{2}{c}{ Value } & Description \\
\hline$L$ & 1 & $\mathrm{~m}$ & Link length \\
$m$ & 1 & $\mathrm{~kg}$ & Link mass \\
$K_{\mathrm{m}}$ & 0.04 & $\mathrm{~N} \mathrm{~m} \mathrm{~A}^{-1}$ & Motor constant \\
$R_{\mathrm{e}}$ & 1 & $\Omega$ & Motor electrical resistance \\
$n$ & $1: 33$ & & Gear reduction ratio \\
$K$ & 30 & $\mathrm{~N} \mathrm{~m}^{-1}$ & Impedance controller stiffness \\
$D$ & 3 & $\mathrm{~N} \mathrm{~s} \mathrm{~m}^{-1}$ & Impedance controller damping \\
\hline$k_{1}$ & 15 & $\mathrm{~N} \mathrm{~m} \mathrm{rad}^{-1}$ & Optimal stiffness joint 1 \\
$q_{0,1}$ & 0.5 & $\mathrm{rad}^{-1}$ & Optimal rest length joint 1 \\
$k_{2}$ & 2.6 & $\mathrm{~N} \mathrm{~m} \mathrm{rad}^{-1}$ & Optimal stiffness joint 2 \\
$q_{0,2}$ & 1.95 & $\mathrm{rad}$ & Optimal rest length joint 2 \\
\hline
\end{tabular}

Table 2.2 - Parameters of the 2-DoF pick-and-place robot arm with parallel springs.

performance of this controller will be rather poor, with no feed-forward torque for acceleration, but the end-effector does follow the trajectory well enough to show the effect of parallel springs.

A simple linear spring is added to both joints, according to (2.61). The motor torque is set to

$$
\tau_{\text {motor }, i}=\tau_{i}-K_{i}\left(q_{i}-q_{0, i}\right)
$$

such that the total torque applied on the joint is always $\tau_{i}$.

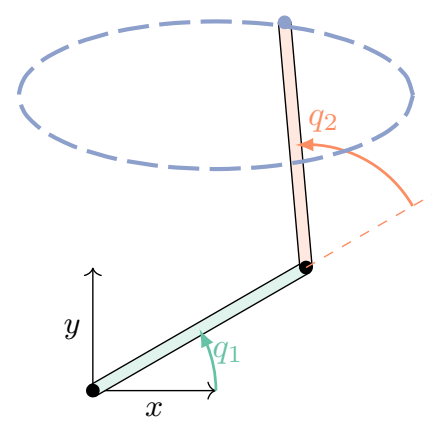

Figure 2.23 - A simple pick-and-place 2-DoF robot arm whose end-effector moves along an elliptical trajectory.

With a spring stiffness of $k_{i}=0$, i.e. without a parallel spring, both joints' motors show peaks in power loss of up to $200 \mathrm{~W}$ (Figure 2.24). The average power loss for the two motors is $50 \mathrm{~W}$ and $28 \mathrm{~W}$, respectively. 


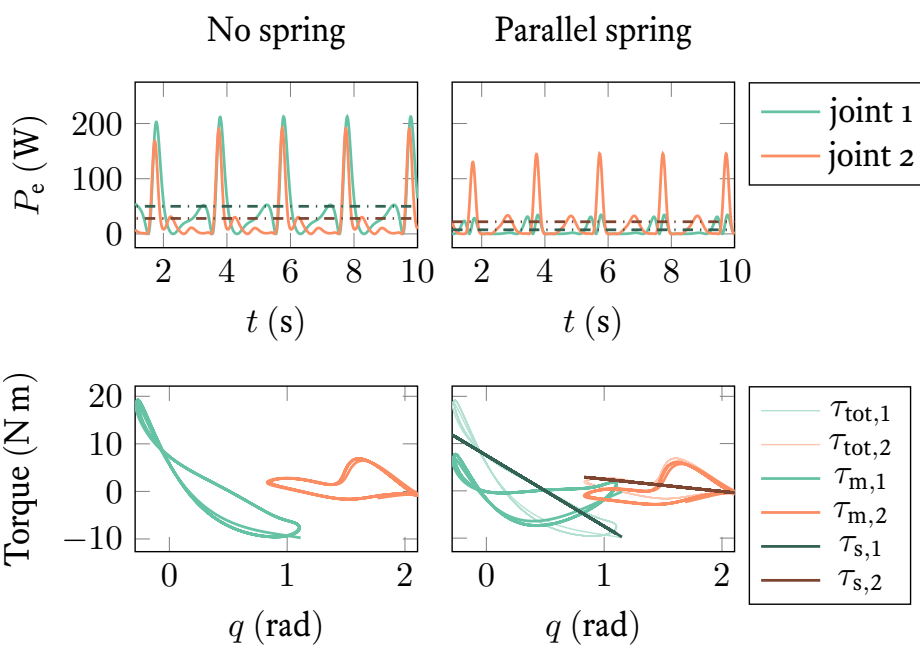

Figure 2.24 - Simulation of the pick-and-place robot arm that executes a periodic trajectory. The power plotted is the electrical power loss $i^{2} R_{\mathrm{e}}$; dashed in dark is the average power loss. Adding a parallel spring greatly reduces this loss, especially for the first joint. The motor torque is lowered by the spring torque, seen in the bottom-right.

From the joint-space plots of both motors, it can already be seen that especially motor 1 delivers a torque that is largely proportional to its rotation, which means that a linear parallel spring could supply most of the work. An numerical optimisation on parameters $k_{i}$ and $q_{0, i}$ that minimises the objective function (2.65) gives two optimal parallel springs as listed in Table 2.2.

$$
J=\int_{0}^{T}\left(P_{\mathrm{e}, 1}+P_{\mathrm{e}, 2}\right) \mathrm{d} t
$$

From the plots on the right hand side of Figure 2.24 it is clear that the springs significantly reduce the energy loss: for motor 2 from $28 \mathrm{~W}$ to $22 \mathrm{~W}$, a moderate improvement, but for motor 1 from $50 \mathrm{~W}$ to $7 \mathrm{~W}$. Overall, this means the energy loss is reduced by $63 \%$. Moreover, the maximum torque delivered by the motors has been lowered, which allows for lighter and smaller motors to be used.

In [107], the use of nonlinear parallel springs is investigated on a realistic pick-and-place 2-DoF robot arm, where real-world experiments show an efficiency improvement of $20 \%$. In [35], a similar approach of tuning elastic elements to increase efficiency is used on a walking biped. 〈example end

In [45] it was shown that the inclusion of parallel springs in the MITCheetah quadruped robot would lead to a reduction in power consump- 
tion by over $50 \%$. Hunt et al. [68] have obtained very efficient resonancebased kangaroo-like walking robots by applying parallel springs.

\section{Serial spring configuration}

One way to make the motor operate in a more efficient region is by including a large gear reduction. While this solves the problem of low speed, high torque applications, it comes with two major drawbacks: first, gearboxes with high ratios often have a rather low efficiency; second, the reflected motor inertia is scaled by $n^{2}$, which may lead to inefficient power transfer (through bad inertia matching), low bandwidth and bad interaction behaviour. Indeed, the motor can become practically nonbackdrivable, which makes impedance or even torque control infeasible. Even if the motor is backdrivable, the friction behaviour of a gearbox is typically nonlinear, which makes precise torque control difficult.

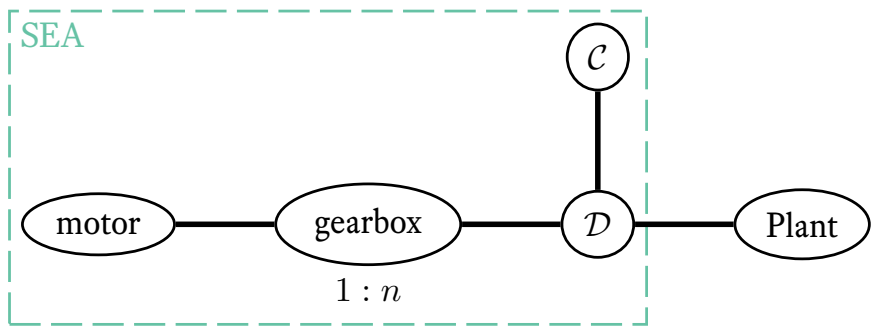

Figure 2.25 - A Series Elastic Actuator allows the motor to operate in a more efficient region, but more importantly, it allows for precise force control and physical, reversible energy storage. The Dirac structure is such that $\tau_{\text {gearbox }}=\tau_{\text {out }}=\tau_{\text {spring }}$ and consequently $\omega_{\text {spring }}=$ $\omega_{\text {gearbox }}-\omega_{\text {out }}$.

The solution to most of these problems is to connect a spring in series with the motor, resulting in a Series Elastic Actuator, as introduced by [110]. A Port-Hamiltonian representation is show in Figure 2.25. Because the output torque is determined purely by the spring state and stiffness, according to (2.66), force control is transformed into an (easier) position control problem.

$$
\tau_{\text {out }}=K \cdot\left(q_{\text {out }}-q_{\text {motor }} / n\right)
$$

If the gear reduction ratio is sufficiently high, such that the motor is practically nonbackdrivable, any static holding torque - that is to say, $\dot{\tau}_{\text {out }}=0$ and $\dot{q}_{\text {out }}=0$ - can be sustained with zero power draw by the motor.

Another benefit of SEA is that the (large, reflected) motor inertia is decoupled from the output. Therefore any impacts on the robot, for 
example ground contact events in a walking robot, are absorbed by the spring. On the one hand this makes the robot safer and lowers energy loss; on the other hand there is less strain on the gearbox and motor.

However, the decoupling between motor and output also mean that the achievable bandwidth is much lower. This is most easily seen by considering the classical transfer function from motor torque $\tau_{M}$ to load angle $\theta_{\mathrm{L}}$ for the simplified system of Figure 2.26:

$$
\begin{aligned}
J_{\mathrm{L}} s^{2} \Theta_{\mathrm{L}} & =K\left(\Theta_{\mathrm{M}}-\Theta_{\mathrm{L}}\right) \\
J_{\mathrm{M}} s^{2} \Theta_{\mathrm{M}} & =-K\left(\Theta_{\mathrm{M}}-\Theta_{\mathrm{L}}\right)+T_{\mathrm{M}} \\
\Theta_{\mathrm{L}} & =\frac{K}{\left(J_{\mathrm{M}} s^{2}+K\right)\left(J_{\mathrm{L}} s^{2}+K\right)-K^{2}} T_{\mathrm{M}} \\
& =\frac{1}{\left(J_{\mathrm{L}} J_{\mathrm{M}} s^{2} / K+\left(J_{\mathrm{M}}+J_{\mathrm{L}}\right) s^{2}\right.} T_{\mathrm{M}} .
\end{aligned}
$$

For $K \rightarrow \infty$, the transfer function reduces to $\frac{1}{\left(J_{\mathrm{M}}+J_{\mathrm{L}}\right) s^{2}}$, i.e. a directdrive. With a low $K$, however-which is whence the SEA derives its benefits - extra low-pass behaviour is introduced by the term $J_{\mathrm{L}} J_{\mathrm{M}} s^{2} / K$.

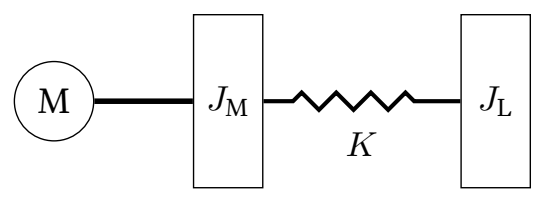

Figure 2.26 - A simplified SEA model with a spring between motor and load inertia. The bandwidth of this system is - depending on $K$-much lower than a direct-drive actuation.

\section{$\S 4.2$ Variable stiffness}

From the previous section it is clear that adding physical storage elements, especially in the form of springs, can be very beneficial for energyefficiency, precise torque control, impact absorption et cetera. However, the optimal stiffness very much depends on the application.

In the case of parallel elastic actuation, a spring that is used to compensate gravity in a robot arm only counteracts the robot's weight in a specific configuration, or at best in a limited range of arm positions. In series elastic actuation, there is a trade-off between desiring a low spring stiffness for more precise force control and better impact absorption, versus a higher stiffness for more precise position control and higher bandwidth.

In highly dynamic applications, such as running legged robots, or robot arms executing fast, periodic motions, the spring stiffness must be 
tuned to the robot inertia and the desired period of the motion.

It follows that the spring stiffness can be optimised for a certain situation, trajectory, or application; but once the robot deviates from this nominal behaviour, a different stiffness would be optimal. With a variable stiffness, the stiffness can be tuned so it is optimal in every situation ([16o]). Therefore, the concept of actuators with an adaptable stiffness, called Variable Stiffness Actuators or VSAs, has received much attention recently. We refer to $[58,156,157,166]$ for extensive reviews of the state-of-the-art in VSAs.

A VSA has a physical spring and a means to change the stiffness felt at the output of the actuator. To distinguish VSAs from Variable Spring Mechanisms, they are also understood to have an actuator capable of injecting energy into the system. The output port of the VSA is given by the power pair $\left(\tau_{\text {out }}, \dot{r}\right)$, with $\tau_{\text {out }}$ the torque at the output and $r$ the output displacement. Because the spring is most often in a serial configuration, the VSA's output is the torque. The apparent output stiffness is a local property defined as

$$
k_{\text {out }}=\frac{\delta \tau_{\text {out }}}{\delta r} .
$$

Example 2.12. In its simplest form, a VSA can be represented by a linear spring with mechanical control port "p1", a stiffness-changing control port "p2" and an interaction or output port $\left(\tau_{\text {out }}, \dot{r}\right)$. The Port-Hamiltonian System of this VSA is given by

$$
\begin{gathered}
\mathcal{H}(x)=\frac{k}{2} s^{2} \\
\dot{x}=\left(\begin{array}{c}
\dot{s} \\
\dot{k}
\end{array}\right)=(0) \frac{\partial \mathcal{H}}{\partial x}(x)+\left(\begin{array}{lll}
1 & 0 & 1 \\
0 & 1 & 0
\end{array}\right)\left(\begin{array}{c}
\dot{q}_{1} \\
\dot{q}_{2} \\
\dot{r}
\end{array}\right) \\
y=\left(\begin{array}{c}
\tau_{1} \\
\tau_{2} \\
\tau_{\text {out }}
\end{array}\right)=\left(\begin{array}{ll}
1 & 0 \\
0 & 1 \\
1 & 0
\end{array}\right)\left(\begin{array}{c}
k s \\
s^{2} / 2
\end{array}\right),
\end{gathered}
$$

where the state $x=(s, k)^{\top}$ consists of both the spring's strain $s$ and its stiffness $k$. The interaction ports are $\left(\tau_{1}, \dot{q}_{1}\right)$, which may inject mechanical power into the spring; $\left(\tau_{2}, \dot{q}_{2}\right)$, the stiffness-changing port; and $\left(\tau_{\text {out }}, \dot{r}\right)$, the output port.

Note that the input-state-output map $g$ need not be constant as in this example; nor have the two control ports to be decoupled-the choice is made in this example such that port 1 only injects mechanical energy, through the direct coupling from $\dot{q}_{1}$ to $\dot{s}$; and port 2 only changes the stiffness, through $\dot{k}=\dot{q}_{2}$.

From these PHS equations it is apparent that changing the stiffness is 
not "free": there is an energy cost from the power injected at port 2,

$$
P_{2}=\tau_{2} \dot{q}_{2}=g_{2}^{\top} \frac{\partial \mathcal{H}}{\partial x}(x) \dot{q}_{2}=\frac{s^{2}}{2} \dot{k} .
$$

Only when the spring is unloaded there is no energy cost associated with changing $k$. (This can be a drawback, but also be exploited; see [47].)

It is of course possible to control $\dot{q}_{1}$ and $\dot{q}_{2}$ in such a way that the internal energy of the VSA is not changed, as long as the output does not move $(\dot{r}=0)$ :

$$
P_{1}=-P_{2} \Rightarrow k s \dot{q}_{1}=-\frac{s^{2}}{2} \dot{q}_{2} \Rightarrow \dot{q}_{1}=-\frac{s}{2} \dot{q}_{2} .
$$

If a real VSA can be constructed or controlled in such a way that the energy cost associated with changing the stiffness is always o, we call that VSA energy-efficient.

$\langle$ example end $\rangle$

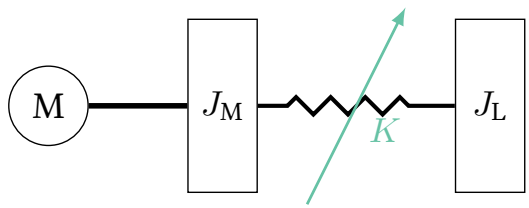

(a) The actual physical stiffness of the compliant element may be changed.

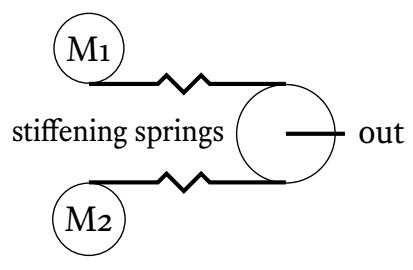

(b) An antagonistic motor setup with stiffening (non-linear) springs results in an output stiffness depending on the preload.

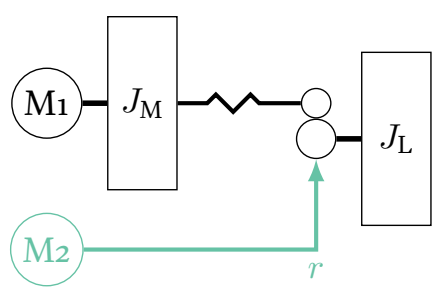

(c) A mechanism, usually a variable gear ratio between spring and output, can change the apparent output stiffness of the spring.

Figure 2.27 - Three ways of achieving a variable-stiffness series elastic element in a Variable Stiffness Actuator (VSA).

A VSA generally has two motors, because the output force of the spring has two degrees of freedom, $r_{0}$ and $k$. The two motors can be configured in two ways ([166]): 
1. an antagonistic motor setup, as in Figure 2.27b;

2. an independent motor setup, as in Figure 2.27c.

The stiffness can be varied by means of three methods:

1. variation of the spring preload, as in Figure 2.27b;

2. variation of the transmission ratio between spring and output (Figure 2.27C);

3. influence of the spring's physical properties (Figure 2.27a).

These stiffness-changing methods can all be combined with either of the motor configurations; Figure 2.27 shows three of the six possible combinations. We will focus on the configurations of Figure $2.27 \mathrm{C}$ and $2.27 \mathrm{~b}$, because they are used often and are interesting from an energy perspective.

\section{Antagonistic stiffening springs}

The VSA configuration of Figure 2.27b features two antagonistic motors, connected to the output pulley by nonlinear, stiffening springs. The motor positions are denoted by $q_{i}$, output position by $r$ and spring states by $s_{i}$. In spite of what the drawing suggests, both motors are connected such that a positive $q_{i}$ means a compression of the spring. The powerconjugate torques of each port are $\tau_{i}$ for the motors and $\tau_{\text {out }}$ for the output link.

It is known that to obtain a linear output stiffness, the spring force must be quadratic:

$$
\tau_{i}=-k_{i} s_{i}\left|s_{i}\right|=-k_{i} s_{i}^{2},
$$

where the simplification can be made because in an antagonistic spring set-up the springs are always loaded in extension, i.e., $s_{i}>0$.

The apparent output stiffness and the energy storage of the actuator can be studied in the Port-Hamiltonian formulation of this system. The state vector is $x=\left(s_{1}, s_{2}\right)^{\top}$ and the system has three power ports: $\left(\tau_{1}, \dot{q}_{1}\right),\left(\tau_{2}, \dot{q}_{2}\right)$ and $\left(\tau_{\text {out }}, \dot{r}\right)$.

$$
\begin{aligned}
\mathcal{H}(x) & =\frac{k}{3}\left(\left(s_{1}\right)^{3}+\left(s_{2}\right)^{3}\right) \\
\left(\begin{array}{c}
\dot{s}_{1} \\
\dot{s}_{2}
\end{array}\right) & =(0) \frac{\partial \mathcal{H}}{\partial x}(x)+\left(\begin{array}{ccc}
-1 & 0 & -1 \\
0 & -1 & 1
\end{array}\right)\left(\begin{array}{l}
\dot{q}_{1} \\
\dot{q}_{2} \\
\dot{r}
\end{array}\right) \\
\left(\begin{array}{c}
\tau_{1} \\
\tau_{2} \\
\tau_{\text {out }}
\end{array}\right) & =\left(\begin{array}{cc}
-1 & 0 \\
0 & -1 \\
-1 & 1
\end{array}\right)\left(\begin{array}{l}
k\left(s_{1}\right)^{2} \\
k\left(s_{2}\right)^{2}
\end{array}\right)
\end{aligned}
$$


The apparent output stiffness is given by

$$
k_{\text {out }}=\frac{\partial \tau_{\text {out }}}{\partial r}=g_{r}^{\top} \frac{\partial^{2} \mathcal{H}(x)}{\partial x^{2}} g_{r},
$$

where $g_{r}$ denotes the third column of $g$, the mapping from $\dot{r}$ to $\dot{s}$. The stiffness is found to be

$$
k_{\text {out }}=\left(\begin{array}{ll}
-1 & 1
\end{array}\right)\left(\begin{array}{cc}
2 k s_{1} & 0 \\
0 & 2 k s_{2}
\end{array}\right)\left(\begin{array}{c}
-1 \\
0
\end{array}\right)=2 k\left(s_{1}+s_{2}\right) .
$$

If the system starts at rest with $q_{i}=r=s_{i}=0$, then $s_{1}=-q_{1}-r$ and $s_{2}=-q_{2}+r$, and the stiffness expressed in motor positions is

$$
k_{\text {out }}=-2 k\left(q_{1}+q_{2}\right) .
$$

From $s_{i}>0$ follows that $\left(q_{1}+q_{2}\right)<0$ and thus this output stiffness is always positive and linearly dependent on the total pretension $q_{1}+q_{2}$. The equilibrium position $r_{0}$ of the output is given by

$$
\left.\tau_{\text {out }}\right|_{r=r_{0}}=0 \Rightarrow k\left(-q_{1}-r_{0}\right)^{2}=k\left(-q_{2}+r_{0}\right)^{2} \Rightarrow r_{0}=\frac{q_{2}-q_{1}}{2},
$$

which is the average differential motor position: a simple linear coordinate transformation on $q_{1}$ and $q_{2}$ to their sum and difference gives direct control inputs for $k_{\text {out }}$ and $r_{0}$.

While the derivations show that an easily controllable VSA can be realised in this way, it is also apparent that there is always a load on the motor, even if $\tau_{\text {out }}=0$, due to the preload on the springs:

$$
\tau_{i}=-k\left(s_{i}\right)^{2} \underset{r=r_{0}}{=} k\left(-\frac{1}{2}\left(q_{1}+q_{2}\right)\right)^{2}=k\left(-\frac{1}{2}\left(-\frac{k_{\text {out }}}{2 k}\right)\right)^{2}=\frac{k_{\text {out }}^{2}}{16 k} .
$$

Considering the significant electrical energy loss in motors, energyefficiency-wise this is not an ideal situation. Furthermore, not all energy stored in the springs can be used by the system: the output can extract energy until the minimum of $\mathcal{H}$ for $r$, i.e. until

$$
\frac{\partial \mathcal{H}}{\partial r}=g_{r}^{\top} \frac{\partial \mathcal{H}}{\partial x}(x)=0 \Rightarrow k\left(s_{1}\right)^{2}=k\left(s_{2}\right)^{2} \Rightarrow r=r_{0} .
$$

However, in the equilibrium position $r=r_{0}$ there is still energy in the actuator, namely

$$
\left.\mathcal{H}\right|_{r=r_{0}}=\frac{2 k}{3}\left(-\frac{q_{1}+q_{2}}{2}\right)^{3}=\frac{2 k}{3}\left(\frac{k_{\text {out }}}{4 k}\right)^{3} .
$$

Due to strain or stress limits caused by the mechanism or physical properties of the springs, there is a maximum to the energy that can be stored 
in each spring. This means that the higher the output stiffness, the less energy storage is available to the system coupled to the actuator:

$$
\mathcal{H}_{\text {available }}=\mathcal{H}_{\max }-\mathcal{H}_{r=r_{0}} \text {. }
$$

\section{Variable transmission}

The variable-transmission VSA as shown in Figure $2.27 \mathrm{C}$ is promising from an energy perspective, because the output is coupled directly to the spring, as is the main motor M1. This means that all of the energy storage capacity of the spring can be used; the system truly behaves as a series elastic actuator with variables spring stiffness. Furthermore, the stiffness changing mechanism for $\mathrm{M}_{2}$ can be designed such that it is completely decoupled from the spring, input and output, such that the stiffness can be changed using minimal - theoretically even o-energy.

We shall analyse a 1-DoF VSA with a variable transmission by studying properties of the Dirac structure that defines the interconnection between the motors, internal states and output. In Visser, Carloni and Stramigioli [159], the analysis is done for $n$-DoF VSAs and used to design an energy-efficient Variable Stiffness Actuator.

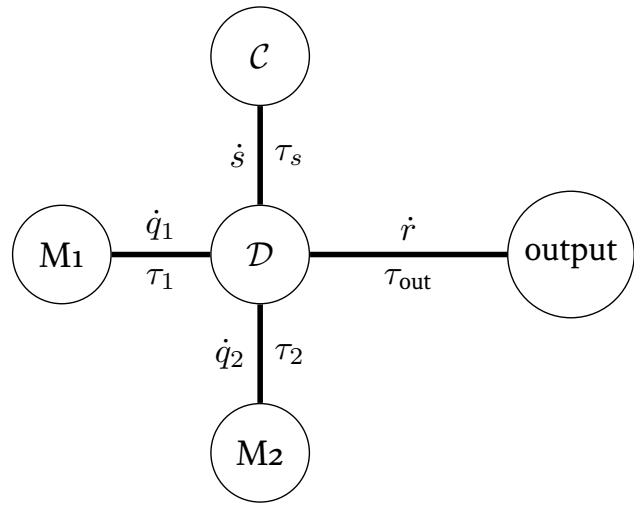

Figure 2.28 - Port-Hamiltonian representation of the variable-transmission type VSA. There is only one storage element, the spring, but the Dirac structure has an internal degree of freedom, $q$, that represents the variable transmission between spring and output.

The drawing in Figure 2.28 defines all the port variables of the VSA. Their interconnection is determined by the Dirac structure $\mathcal{D}$, which may depend on the output position $r$ and some internal degree of freedom of the mechanism, $q$ that may in turn depend on $q_{i}$ or $s$. A matrix representation of the Dirac structure is given in (2.85). Each $\alpha_{i}$ may be $\alpha_{i}(q, r)$. The lower-right part of the matrix is filled with zeros, because 
that part describes a gyrating effect between the mechanical ports, which is impossible. The matrix is skew-symmetric so the interconnection is power-continuous.

$$
\left(\begin{array}{c}
\dot{s} \\
\tau_{1} \\
\tau_{2} \\
\tau_{\text {out }}
\end{array}\right)=\left[\begin{array}{cccc}
0 & \alpha_{1} & \alpha_{2} & \alpha_{r} \\
-\alpha_{1} & 0 & 0 & 0 \\
-\alpha_{2} & 0 & 0 & 0 \\
-\alpha_{r} & 0 & 0 & 0
\end{array}\right]\left(\begin{array}{c}
\tau_{s} \\
\dot{q}_{1} \\
\dot{q}_{2} \\
\dot{r}
\end{array}\right)
$$

With this interconnection matrix, the PHS equations of the system with state $x=(s, q)^{\top}$ and external ports for M1, M2 and the output are:

$$
\begin{aligned}
\mathcal{H} & =\frac{k}{2} s^{2} \\
\left(\begin{array}{c}
\dot{s} \\
\dot{q}
\end{array}\right) & =(0) \frac{\partial \mathcal{H}}{\partial x}(x)+\left(\begin{array}{lll}
\alpha_{1} & \alpha_{2} & \alpha_{r} \\
\beta_{1} & \beta_{2} & \beta_{r}
\end{array}\right)\left(\begin{array}{c}
\dot{q}_{1} \\
\dot{q}_{2} \\
\dot{r}
\end{array}\right) \\
\left(\begin{array}{c}
\tau_{1} \\
\tau_{2} \\
\tau_{\text {out }}
\end{array}\right) & =\left(\begin{array}{ll}
\alpha_{1} & \beta_{1} \\
\alpha_{2} & \beta_{2} \\
\alpha_{r} & \beta_{r}
\end{array}\right) \frac{\partial \mathcal{H}}{\partial x}(x),
\end{aligned}
$$

where $\beta_{i}$ determines the mapping of inputs to the internal configuration of the VSA.

In order to separate the functions of $\mathrm{M}_{1}$ and $\mathrm{M}_{2}$ into a pure power source and pure stiffness changing motor, respectively, $\dot{q}_{2}$ must be decoupled from $\dot{s}\left(\alpha_{2}=0\right)$ and $\dot{q}$ must only depend on $\dot{q}_{2}\left(\beta_{1}=\beta_{r}=0\right)$. If the mechanism can be constructed in such a way, the result is a perfectly energy-efficient VSA with the following properties. by

The apparent output stiffness around a certain position $r=\bar{r}$ is found

$$
k_{\text {out }}(q, r=\bar{r})=g_{r}^{\top} \frac{\partial^{2} \mathcal{H}}{\partial x^{2}} g_{r}=\left(\alpha_{3}(q, \bar{r})\right)^{2} k
$$

and thus the output stiffness can be changed if the mechanism is such that $\alpha_{3}$ depends on $q$, i.e., such that the transmission ratio between output and spring is variable; $\dot{q}=\beta_{2} \dot{q}_{2}$. The energy cost associated with a stiffness change is

$$
P_{2}=\tau_{2} \dot{q}_{2}=g_{2} \frac{\partial \mathcal{H}}{\partial x}(x) \dot{q}_{2}=\left(\begin{array}{ll}
0 & \beta_{2}
\end{array}\right)\left(\begin{array}{c}
k s \\
0
\end{array}\right) \dot{q}_{2}=0 .
$$

Finally, the equilibrium position of the output $r_{0}$ when $\tau_{\text {out }}=0$ depends on $\alpha_{1}$ and $\alpha_{3}$, but is determined by $\mathrm{M}$. 


\section{§4.3 Morphological computation}

The concept of control by physical interconnection can be taken further by pushing the boundary between control and system even farther towards the physical system. The "computation" that is traditionally carried out by a computer running control algorithms is mostly - or even completely-executed in the controlled system. This sharing of control of the robot between "traditional" control and the morphology of the robot itself is called morphological computation, introduced by [104]

In general, morphology may refer to only the shape of the mechanical system, in which case there is only kinematic morphological computation. A famous example of this is the Strandbeest [75], which contains a mechanism that converts a continuous rotating motion into a walking leg motion. This and similar mechanisms were analysed in [137]. While a well-designed kinematic morphology results in kinematic morphological computation, it is "merely" good mechanism design and not novel. (Mechanisms designed by Pafnuty Chebyshev in the 19th century are still widely used in locomotion.)

On the other hand, morphology may also refer to the dynamic properties of the robot, such as mass distribution and compliance. This leads to dynamical morphological computation, which not only simplified control but can also lead to high energy efficiency. After all, whereas in traditional control theory the dynamics of a system are cancelled out as much as possible, to steer the system's states to a desired point or along a desired trajectory, in dynamic morphological computation the inherent dynamics of the system are the desired dynamics, so the natural behaviour of the system already follows the desired trajectories. Successful examples of dynamic morphological computation may be found in [108] or [72], quadrupedal robots with almost-passive locomotion behaviour.

One possible approach to morphological computation is biomimetic morphological computation, where "proper" morphology is observed in nature and translated to morphological concepts for robots, as in [50]. (Parts of) the field of Soft Robotics may also be considered as morphological computation; see [78]. At the same time, an attempt is made at a proper theoretical foundation for morphological computation, viz. [62] and [49].

In dynamic morphological computation, Port-Hamiltonian System theory and energy-based robotics can make an important contribution. After all, morphological computation is concerned with properly interconnected physical storage elements (masses, springs). In Port-Hamiltonian System theory, those storage elements and their interconnection are $e x$ plicitly modelled by their energy functions and the Dirac structure or internal interconnection matrix $(J)$. Therefore, modelling the combination of system and controller as interconnected PHSs gives direct insight 
into the dynamic morphology. Furthermore, the distinction between "controller" and "system" completely disappears, since both are described, modelled and designed as a PHS. And if the system's inherent, natural dynamics exhibit desired behaviour, the control system merely needs to inject energy to keep the motion going and compensate for e.g. friction losses, and possibly stabilise the oscillations.

The energy-based modelling and control approach is a perfect match for designing, analysing and understanding morphological computation. The application of Port-Hamiltonian System theory and energy-based robotics to the field of morphological computation was first studied in [46], where a fully elastic spine was shown to generate locomotion in a quadruped, with control limited to energy injection through the 1-DoF legs. 


\section{$\S 5$ Conclusion}

Robots, like all physical systems, are governed by energy. Interaction of the actuators with the mechanical system of the robot, and interaction of the robot with the environment, is characterised by energy exchange. Describing systems and controllers in an energy-based way gives more insight into these energy flows in the physical system. In this work, we have shown how energy-based theories and tools can be applied to the field of robotics.

We have used the Port-Hamiltonian System theory to model the robot with energy at the centre. We have shown methods to measure and control energy flows in practical systems. Energy-based thinking can also be used for controller design: whether in control by interconnection, where the control algorithms are "virtual physical systems" in PortHamiltonian fashion; or in control by physical interconnection, where the mechanical design of the robot is based on the desired energetic behaviour.

This energy-based view of the mechanical system, actuators and control algorithms dissolves the traditional boundaries between them: it is irrelevant whether a behaviour is the result of a physical process, or of a virtual process. It is a holistic, energy-based view of the robot. 


\section{Chapter 3}

\section{Hoppers}

Quadrupedal running is an efficient form of locomotion found in nature, which serves as an inspiration for robotics. We believe that a resonancebased approach is the path towards energy-efficient legged locomotion and running robots. The first step in working towards this goal is creating an energy-efficient one-legged hopper. Such a one-legged hopper was designed and constructed. The impact efficiency of the mechanism is calculated analytically, determined in simulation and measured with the prototype. The impact efficiency as calculated from the experiments is found to be in agreement with the analytical expectation and simulation results. Finally, using an electric motor to inject energy by creating a virtual spring with reverse hysteresis, hopping is achieved. 


\section{§1 Introduction}

Quadrupedal running consists of coordinated periodic motions that, in nature, form a fast and energy-efficient bound or gallop. This form of locomotion has been an inspiration for the field of robotics for years. However, we have still not achieved the graceful, fast movement; nor matched this energy-efficiency in quadrupedal robots. We believe one of the steps towards this goal is to exploit the periodicity of the locomotion, by using resonance to generate the motions and improve energy-efficiency: just like in nature [4].

Animals have a certain degree of elasticity in their tendons and muscles [7]. As such, the stiffness is also incorporated in models of walking, for example in the SLIP model $[50,51]$. The compliance in legs is useful to absorb shocks from impact, as in MIT's cheetah, in which elastic tendons introduce compliance in the leg mechanism [130, 45]. Another option to introduce compliance is to actuate stiff members in a compliant way, for example with pneumatics as in HyQ[129]. The stiffness of animal legs has been related to their movement speed [87] and energetic cost of locomotion [117]. This has led other researchers to investigate the application of variable stiffness actuators and mechanisms for use in legged locomotion [70, 162].

To take the concept of compliance in legs further, it would be beneficial to the energy-efficiency if this compliance could be used to facilitate the periodic motion required to move forward. In the field of morphological computation, research is ongoing towards exploiting the body dynamics in achieving desired behaviour [103]. For example, special controllers that make use of the passive dynamics of a system are investigated $[155,20,72]$. Others use actuators that introduce high-frequency oscillation and try to find mechanisms that then effectuate forward motion [85]. Some analysis on the energy efficiency of a vibrating elastic robot has been done [169]. In spite of all this effort, we have yet to reach the high energy-efficient performance that is seen in nature.

Our goal is to create a robot that can run efficiently and with grace, like a real cheetah does. We intend to use a flexible spine that, combined with front and rear legs, creates the periodic motion seen in animals. Previous research has shown that a spinal joint with a spring can improve the overall energy-efficiency of the robot [45]. Others have shown that a spine with nonlinear stiffness leads to even higher efficiency [77]. In our robot, the front and rear body will be made to bounce on their legs, while the flexible spine introduces a resonance that results in forward motion. For maximally energy-efficient actuation, the front and rear 'hoppers' will be actuated in resonance with a controlled offset in their phase, as in [47]. A crucial part of such a resonance-based robot is thus an energy-efficient bouncing leg, i.e. the 'hopper'. 
This chapter is about the design and analysis of such a one-legged hopper. The design considerations of the mechanism are discussed and the used prototype is described; then the energy loss is investigated in both a mathematical framework and in practice and is shown to be in accordance with the expectations.

\section{$\S 2$ Design}

In order to be useful in resonance-based locomotive systems, the leg should have passive dynamics that allow for hopping at resonance. Important features are the parallel spring, low weight and minimal impact loss, as detailed in the following section.

\section{$\S 2.1$ Design Considerations}

Our goal for the robot is to have a natural bounding gait, ideally with a leg step frequency of about $1 \mathrm{~Hz}$ so that the movement is clearly visible. During the hopping, energy will be lost due to friction and impact on the ground. This energy loss needs to be compensated to sustain continuous hopping. The stance phase is the only period during which energy can be introduced into the system. To allow enough energy to be introduced, we aim for a long stance phase of 50\% of the step period.

Assuming the air phase is parabolic, a flight time of half a second implies an initial vertical velocity of $v_{0}=g t / 2=2.45 \mathrm{~m} \mathrm{~s}^{-1}$. With this vertical velocity, a height of about $30 \mathrm{~cm}$ would be reached, regardless of the system's mass. A small bench top prototype is desired for testing, so a jump height of $30 \mathrm{~cm}$ is considered unpractical. To lower the height, an increase of the stepping frequency is required.

The designed one-legged hopper will be a planar mechanism. This means it can be light, so that the required power and forces in the mechanism are as low as possible. To keep the number of joints (and thereby the amount of friction) low, a parallelogram construction is chosen. This ensures that the leg stays perpendicular to the ground, resulting in a well-defined ground contact point. A spring placed in the diagonal of the parallelogram allows energy storage during compression of the leg. To be able to compensate for the friction and impact losses, a DC motor can be attached to one of the joints with minimal impact on the mechanism's behaviour.

A backdrivable parallel elastics configuration is obtained, so no holding torque is required to be able to store energy in the spring and, therefore, it also allows the leg to function without the motor. 


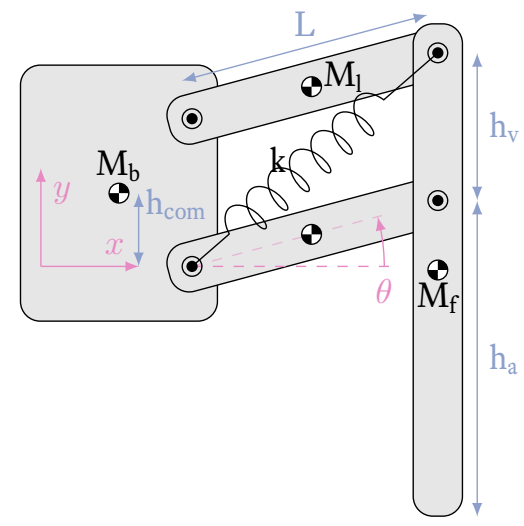

Figure 3.1 - Schematic diagram of the one-legged hopper. The three degrees of freedom are $x$ and $y$ for the translation of the body and the angle $\theta$ of the leg. The relevant parameters are listed in Table 3.1.

\section{$\S 2.2$ System}

The prototype is lasercut from DELRIN ${ }^{\mathrm{TM}}$ (Polyoxymethylene). The low friction coefficient of this material allows efficient plain bearings to be created by inserting metal rods through smooth drillholes. The thickness for all structural members is $6 \mathrm{~mm}$, the driving axle is a $\varnothing 6 \mathrm{~mm}$ brass cylinder and the other joints are made with $\varnothing 3 \mathrm{~mm}$ polished steel cylinders.

The prototype is attached to a $33 \mathrm{~cm}$ long aluminium L-profile (20x20x2mm), which pivots at the other end to allow upwards motion of the mechanism while constraining the other degrees of freedom. A small DELRIN ${ }^{\mathrm{TM}}$ cylinder with a diameter of $2.5 \mathrm{~mm}$ is used to reduce the ground contact area and minimise friction.

A relatively low transmission ratio of $1: 2$ is chosen between motor and leg, to allow backdrivability while increasing the torque that can be applied to the rotating elements. The motor used is a Maxon RE-Max 226806, which has a stall torque of $299 \mathrm{mN} \mathrm{m} \mathrm{[3].} \mathrm{The} \mathrm{sensor,} \mathrm{an} \mathrm{AMS}$ AS5045 rotary position sensor, is mounted collocated to the motor[2].

A schematic drawing of the mechanism is shown in Figure 3.1 and a photograph of the actual prototype can be seen in Figure 3.2. Relevant parameter values of the mechanism are listed in Table 3.1. The rotating elements are present in an upper pair and lower pair, but in the table their mass is combined as $\mathrm{M}_{1}$. 
Table 3.1 - Properties of the mechanism.

\begin{tabular}{ccc}
\hline Symbol & Value with motor (without) & Unit \\
\hline $\mathrm{M}_{\mathrm{b}}$ & $370(210)$ & gram \\
$\mathrm{M}_{\mathrm{l}}$ & 28.0 & gram \\
$\mathrm{M}_{\mathrm{f}}$ & 11.5 & gram \\
$\mathrm{L}$ & 8.0 & centimeter \\
$\mathrm{h}_{\mathrm{v}}$ & 6.0 & centimeter \\
$\mathrm{h}_{\mathrm{a}}$ & 7.4 & centimeter \\
$\mathrm{k}$ & $368.6(184.3)$ & newton/meter \\
\hline
\end{tabular}

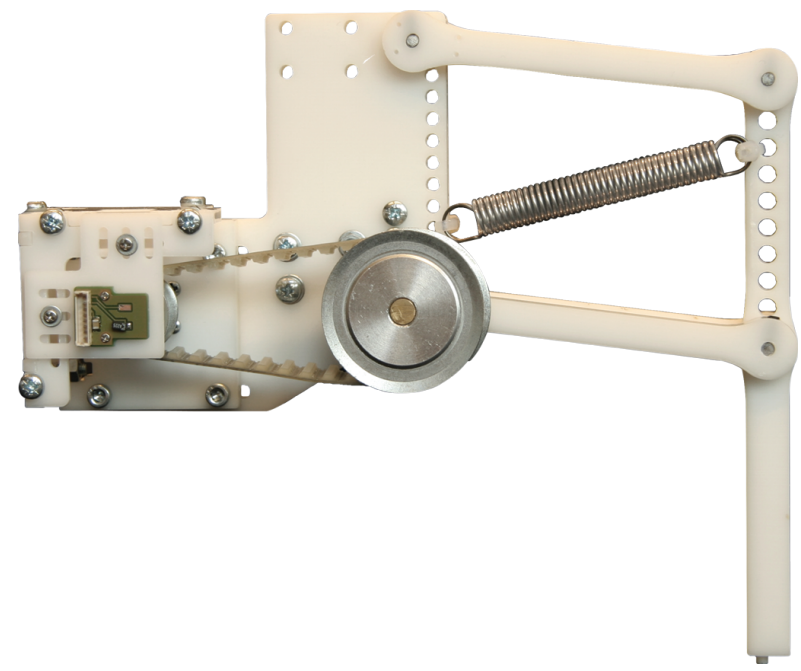

Figure 3.2 - Photograph of the mechanism with the motor mount attached. The green PCB holds the rotary position sensor. The motor is mounted behind the main body element, coaxially to the sensor. 


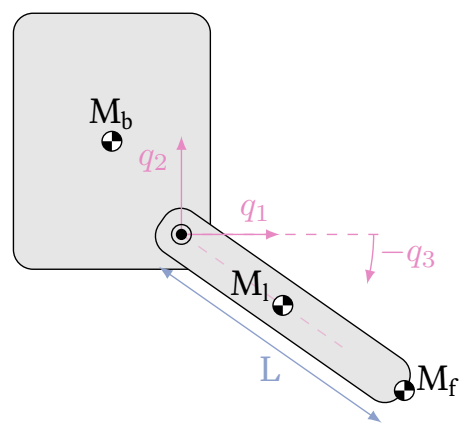

Figure 3.3 - Reduced mechanism for the analytical consideration. The degrees of freedom $q_{1}$ and $q_{2}$ correspond to the translation of the body $x$ and $y$ in Figure 3.1, $q_{3}$ corresponds to the leg angle $\theta$. The relevant parameters are listed in Table 3.1.

\section{$\S 3$ Method}

\section{§3.1 Analytical}

To obtain an analytical description of the system, the Lagrangian equations are used to derive the generalised robotics equation:

$$
\mathbf{M}(\mathbf{q}) \ddot{\mathbf{q}}+\mathbf{C}(\mathbf{q}, \dot{\mathbf{q}}) \dot{\mathbf{q}}+\mathbf{N}(\mathbf{q}, \dot{\mathbf{q}})=\mathbf{F} .
$$

For the analytical derivation of the impact loss, the mechanism is reduced to the one depicted in Figure 3.3. This results in the following terms for $\mathbf{M}, \mathbf{C}$ and $\mathbf{N}$ :

$$
\begin{gathered}
\mathbf{M}(\mathbf{q})=\left[\begin{array}{ccc}
\left(\mathrm{M}_{\mathrm{b}}+\mathrm{M}_{\mathrm{L}}\right) & 0 & -\sin \left(q_{3}\right) \gamma \\
0 & \left(\mathrm{M}_{\mathrm{b}}+\mathrm{M}_{\mathrm{L}}\right) & \cos \left(q_{3}\right) \gamma \\
-\sin \left(q_{3}\right) \gamma & \cos \left(q_{3}\right) \gamma & \mathrm{L}^{2}\left(\frac{1}{4} \mathrm{M}_{\mathrm{l}}+\mathrm{M}_{\mathrm{f}}\right)+\mathrm{I}_{\mathrm{leg}}
\end{array}\right] \\
\mathbf{C}(\mathbf{q}, \dot{\mathbf{q}}) \dot{\mathbf{q}}=\left[\begin{array}{c}
-\dot{q}_{3}^{2} \cos \left(q_{3}\right) \gamma \\
-\dot{q}_{3}^{2} \sin \left(q_{3}\right) \gamma \\
-\dot{q}_{1} \dot{q}_{3} \cos \left(q_{3}\right) \gamma-\dot{q}_{2} \dot{q}_{3} \sin \left(q_{3}\right) \gamma
\end{array}\right] \\
\mathbf{N}(\mathbf{q}, \dot{\mathbf{q}})=\left[\begin{array}{c}
0 \\
g\left(\mathrm{M}_{\mathrm{b}}+\mathrm{M}_{\mathrm{L}}\right) \\
g \cos \left(q_{3}\right) \gamma
\end{array}\right], \quad \mathbf{F}=\left[\begin{array}{c}
F_{\mathrm{x}} \\
F_{\mathrm{y}} \\
\tau
\end{array}\right]
\end{gathered}
$$

where $\gamma=\mathrm{L}\left(\frac{1}{2} \mathrm{M}_{\mathrm{l}}+\mathrm{M}_{\mathrm{f}}\right), \mathrm{I}_{\text {leg }}=\frac{1}{12} \mathrm{M}_{\mathrm{l}} \mathrm{L}^{2}$ and $\mathrm{M}_{\mathrm{L}}=\mathrm{M}_{\mathrm{l}}+\mathrm{M}_{\mathrm{f}}$.

This description of the system can subsequently be used to determine the theoretical impact efficiency. To this end, the Pfaffian constraints 
of the system at ground contact are identified. In ground contact, the system can be described by (3.1) extended with a constraint matrix $\mathbf{A}(\mathbf{q})$ and corresponding constraint force $\lambda$ :

$$
\begin{aligned}
\mathbf{M}(\mathbf{q}) \ddot{\mathbf{q}}+\mathbf{C}(\mathbf{q}, \dot{\mathbf{q}}) \dot{\mathbf{q}}+\mathbf{N}(\mathbf{q}, \dot{\mathbf{q}}) & =\mathbf{F}+\mathbf{A}(\mathbf{q}) \lambda, \\
\mathbf{A}^{\top}(\mathbf{q}) \dot{\mathbf{q}} & =0 .
\end{aligned}
$$

When contact is made with the ground, the foot $\left(x_{\mathrm{f}}, y_{\mathrm{f}}\right)$ does not move any more. The constraint equation for the system is then given by:

$$
\mathbf{A}^{\top}(\mathbf{q}) \dot{\mathbf{q}}=\left[\begin{array}{ccc}
1 & 0 & -\mathrm{L} \sin \left(q_{3}\right) \\
0 & 1 & \mathrm{~L} \cos \left(q_{3}\right)
\end{array}\right]\left[\begin{array}{c}
\dot{q}_{1} \\
\dot{q}_{2} \\
\dot{q}_{3}
\end{array}\right]=\left[\begin{array}{c}
\dot{x}_{\mathrm{f}} \\
\dot{y}_{\mathrm{f}}
\end{array}\right]=\left[\begin{array}{l}
0 \\
0
\end{array}\right] .
$$

The instantaneous impact is considered: let $t^{-}$be the time just before the impact and $t^{+}$the time after the impact. Equation (3.5) can be integrated over time, under the assumption that $\mathbf{q}\left(t^{+}\right) \approx \mathbf{q}\left(t^{-}\right)$, because $t^{+} \approx t^{-}$. This results in:

$$
\begin{aligned}
\mathbf{M}(\mathbf{q}) \dot{\mathbf{q}}\left(t^{+}\right)-\mathbf{M}(\mathbf{q}) \dot{\mathbf{q}}\left(t^{-}\right) & =\mathbf{A}(\mathbf{q}) \int_{t^{-}}^{t^{+}} \lambda(t) \mathrm{d} t \\
\mathbf{A}^{\top}(\mathbf{q}) \dot{\mathbf{q}}\left(t^{+}\right) & =0 .
\end{aligned}
$$

Left multiplying (3.7) by $\mathbf{M}^{-1}(\mathbf{q})$, subsequent left multiplication by $\mathbf{A}^{\top}(\mathbf{q})$ and substitution with (3.8) results in (q as argument left out for clarity):

$$
-\left(\mathbf{A}^{\top} \mathbf{M}^{-1} \mathbf{A}\right)^{-1} \mathbf{A}^{\top} \dot{\mathbf{q}}\left(t^{-}\right)=\int_{t^{-}}^{t^{+}} \lambda(t) \mathrm{d} t .
$$

Substituting (3.9) back into (3.7) allows the expression to be written without the integral:

$$
\begin{array}{r}
\mathbf{M} \dot{\mathbf{q}}\left(t^{+}\right)-\mathbf{M} \dot{\mathbf{q}}\left(t^{-}\right)=-\mathbf{A}\left(\mathbf{A}^{\top} \mathbf{M}^{-1} \mathbf{A}\right)^{-1} \mathbf{A}^{\top} \dot{\mathbf{q}}\left(t^{-}\right), \\
\dot{\mathbf{q}}\left(t^{+}\right)=\left(\mathbf{I}-\mathbf{M}^{-1} \mathbf{A}\left(\mathbf{A}^{\top} \mathbf{M}^{-1} \mathbf{A}\right)^{-1} \mathbf{A}^{\top}\right) \dot{\mathbf{q}}\left(t^{-}\right) .
\end{array}
$$

This result is an explicit equation that relates the velocity $\dot{\mathbf{q}}$ after the impact to the velocity before the impact. By combining this relation with the expression for the kinetic co-energy before and after the impact, a relation for energy loss due to impact is found:

$$
\begin{aligned}
\Delta E_{\mathrm{k}} & =\frac{1}{2} \dot{\mathbf{q}}\left(t^{+}\right)^{\top} \mathbf{M} \dot{\mathbf{q}}\left(t^{+}\right)-\frac{1}{2} \dot{\mathbf{q}}\left(t^{-}\right)^{\top} \mathbf{M} \dot{\mathbf{q}}\left(t^{-}\right) \\
& =-\frac{1}{2} \dot{\mathbf{q}}\left(t^{-}\right)^{\top}\left(\mathbf{A}\left(\mathbf{A}^{\top} \mathbf{M}^{-1} \mathbf{A}\right)^{-1} \mathbf{A}^{\top}\right) \dot{\mathbf{q}}\left(t^{-}\right) .
\end{aligned}
$$


The impact efficiency $\eta$ is expressed as the ratio of energy after impact to energy before impact:

$$
\eta=\frac{E_{\mathrm{k}}^{+}}{E_{\mathrm{k}}^{-}}=\frac{E_{\mathrm{k}}^{-}+\Delta E_{\mathrm{k}}}{E_{\mathrm{k}}^{-}} .
$$

Substituting (3.11) into (3.14) leads to an expression of the form

$$
\eta_{\mathrm{fit}}=v_{0}(A \cos (2 \theta)-B),
$$

where $A$ contains mass terms of the body, and $B$ of the foot-the leg inertia is present in both. This shows that some energy is always lost (the $B$ term), but the kinetic energy loss of the body depends greatly on the leg angle at impact.

\section{$\S 3.2$ Simulation}

The mechanism described in 3.2.2 is modelled as four rigid bodies connected by rotational joints. These are simulated in the modelling and simulation package 20SIM[29]. The properties of the elements are made identical to the values from the prototype.

The ground contact is modelled as a combination of a Hunt-Crossley model [69] for normal force (3.16) and Coulomb friction (3.17).

$$
\begin{aligned}
& F_{y}=K_{\mathrm{c}} \cdot \delta+K_{\mathrm{d}} \cdot|\delta| \cdot \dot{\delta} . \\
& F_{x}=-\mu \cdot F_{y} \cdot \operatorname{sgn}\left(v_{x}\right) .
\end{aligned}
$$

This ground contact is tuned such that a deflection $\delta$ of the foot is less than $1 \mathrm{~mm}$ into the ground and the ground contact is critically damped. A linear guide is simulated by constraining all degrees of freedom but the vertical translation; this guide is attached to either the foot or the body element. The impact efficiency calculation is trivial, as the energy in the system is known.

\section{$\S 3.3$ Real World}

The prototype, as described in Section 3.2.2: System, can also be used to determine the impact efficiency in the real world. For these measurements, the motor was removed and a spring with a lower stiffness was used. Furthermore, a piece of nylon string was attached between the other corners of the parallelogram to configure the impact angle.

The energy loss during the impact cannot be measured directly, but needs to be calculated from other-measurable - values: only the angle $\theta$ can be measured. In the following energy calculations, all mass except the body mass is considered negligible. 
When the mechanism is dropped from a predetermined height $\left(\mathrm{h}_{\mathrm{df}}\right.$, measured from the foot down), the energy before impact is known. Just before impact, at $t=t^{-}$, the total energy $E^{-}$consists of the kinetic energy from the drop, the potential energy from the remaining height and the energy stored in the spring $\left(E_{\mathrm{u}}\right)$ :

$$
\begin{aligned}
E^{-} & =E_{\mathrm{p}}+E_{\mathrm{k}}^{-}+E_{\mathrm{u}} \\
& =\left(\mathrm{h}_{\mathrm{a}}-\mathrm{L} \sin \left(\theta^{-}\right)+\mathrm{h}_{\mathrm{com}}\right) \mathrm{M}_{\mathrm{b}} g+\mathrm{h}_{\mathrm{df}} \mathrm{M}_{\mathrm{b}} g+E_{\mathrm{u}}\left(\theta^{-}\right) .
\end{aligned}
$$

Immediately after impact, at $t=t^{+}$, the only term that is changed (and therefore unknown) is the kinetic energy $E_{\mathrm{k}}^{+}$. An instantaneous change is considered, so the angle is unchanged $\left(\theta^{+} \approx \theta^{-}\right)$:

$$
\begin{aligned}
E^{+} & =E_{\mathrm{p}}+E_{\mathrm{k}}^{+}+E_{\mathrm{u}} \\
& =\left(\mathrm{h}_{\mathrm{a}}-\mathrm{L} \sin \left(\theta^{+}\right)+\mathrm{h}_{\mathrm{com}}\right) \mathrm{M}_{\mathrm{b}} g+E_{\mathrm{k}}^{+}+E_{\mathrm{u}}\left(\theta^{+}\right) .
\end{aligned}
$$

After the impact, the leg continues to compress, up to the point of maximum compression at $t=t^{*}$. At this point the kinetic energy is zero.

$$
\begin{aligned}
E^{*} & =E_{\mathrm{p}}+0+E_{\mathrm{u}} \\
& =\left(\mathrm{h}_{\mathrm{a}}-\mathrm{L} \sin \left(\theta^{*}\right)+\mathrm{h}_{\text {com }}\right) \mathrm{M}_{\mathrm{b}} g+0+E_{\mathrm{u}}\left(\theta^{*}\right) .
\end{aligned}
$$

The energy terms (3.20) and (3.18) can be subtracted from each other which, combined with $\theta^{+} \approx \theta^{-}$, results in:

$$
\begin{array}{r}
E^{+}-E^{*}=\left(-\mathrm{L} \sin \left(\theta^{-}\right)+\mathrm{L} \sin \left(\theta^{*}\right)\right) \mathrm{M}_{\mathrm{b}} g \\
+E_{k}^{+}+E_{\mathrm{u}}\left(\theta^{-}\right)-E_{\mathrm{u}}\left(\theta^{*}\right) .
\end{array}
$$

The assumption is made that there is no energy lost during the compression of the leg from after the impact up to the point of maximum compression:

$$
E^{+} \approx E^{*}
$$

With this assumption, expression (3.21) is equal to zero, which means the term $E_{\mathrm{k}}^{+}$can be expressed as:

$$
\begin{gathered}
E_{\mathrm{k}}^{+}=-\left(-\mathrm{L} \sin \left(\theta^{-}\right)+\mathrm{L} \sin \left(\theta^{*}\right)\right) \mathrm{M}_{\mathrm{b}} g \\
-\left(E_{\mathrm{u}}\left(\theta^{-}\right)-E_{\mathrm{u}}\left(\theta^{*}\right)\right) .
\end{gathered}
$$

This shows that the kinetic energy in the system before the impact and after the impact can be determined by only measuring the leg angle $\theta$. The impact efficiency can again be calculated with (3.14). 


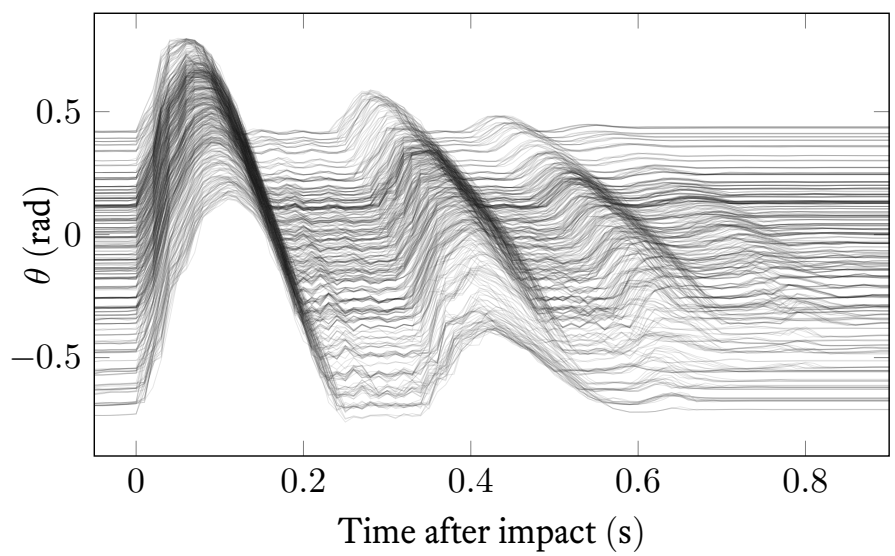

Figure 3.4 - Plot of $\theta$ for all impact measurement tests. Every measurement is represented by one semi-transparant line. The highest peak, roughly around $t=0.1 \mathrm{~s}$ corresponds to $t^{*}$ : the point of maximum leg compression. The subsequent peaks are from bounces on the floor.

\section{$\S 4$ Results}

\section{$\S 4.1$ Impact}

To obtain values of the impact efficiency in the real world, the prototype was dropped as described in subsection 3.3.3. Over 500 experiments were carried out with varying impact angles, logging the angle $\theta$ at $100 \mathrm{~Hz}$.

The measured angle profiles of all these measurements are shown in Figure 3.4. Test runs with equal impact angles show almost identical angle profiles, even though the setup was moved and measurements were performed over a two-day timespan. This shows that the repeatability is very high.

The impact efficiency for each test run is graphed in Figure 3.5a. The analytical impact efficiency, as determined with (3.14) and (3.13), is also plotted. Additionally, a least squares fit of (3.15) was made through the measurement points. This fit is made because the dynamics of the pivoting boom are not considered in the analytical derivation. With slightly changed parameters for $A$ and $B$, this fit aligns well with the measurements. However, the measurements seem to be somewhat skewed compared to the analytical fit.

To investigate the cause of this skew, the dynamic model of Section 3.3.2: Simulation was used to replicate these experiments in simulation. In the theoretical consideration, the leg is unable to slip. However, in the prototype, the body is constrained in rotation but the leg slides 
over the floor during the compression phase. As such, simulations were performed with both the linear guide attached to the leg element and the guide attached to the body element. The obtained impact efficiencies are graphed in Figure 3.5b.

When the linear guide is attached to the foot element, the assumption that no energy is lost during compression (3.22) is correct. However, when the linear guide is attached to the body element, the foot is able to slide over the floor, resulting in energy loss due to friction. This results in the skewed line seen in Figure 3.5b. Surprisingly, the simulated impact efficiencies were higher than expected from the theory. This can be attributed to the visco-elastic ground contact model, which results in less energy loss than the instantaneous impact used in the analytical description.

\section{$\S 4.2$ Hopping}

The goal of the research is to obtain a hopping leg. Therefore, the prototype was also used to attempt this hopping. The purpose of the motor and controller is to inject energy into the system to compensate for friction and impact losses.

Our approach is to use the motor to create a virtual spring with negative hysteresis, similar to [82]: we create the hysteresis not by switching the equilibrium position of the spring, but by changing the virtual spring's stiffness, as illustrated in Figure 3.6. The energy injected is the area enclosed by the curve and is thus directly dependent on the amount of hysteresis. The virtual spring allows us to change the effective leg stiffness and thus change the resonance frequency.

The measurements of the hopping are shown in Figure 3.7. The frequency of hopping is around $2 \mathrm{~Hz}$ so only slightly higher than our original goal of $1 \mathrm{~Hz}$. The hopping achieved in the simulation is also graphed; these results are in close agreement with the measurements.

The hysteresis required for hopping was very large and the stiffness during the compression phase was even negative. An adverse effect of the large hysteresis, combined with the low transmission ratio, is that the motor almost continuously has to deliver maximum torque. This quickly heats up the motor, which means sustained hopping is not possible.

Why this large hysteresis is required is not immediately apparent. The impact efficiency is obviously very good, as seen in Figure 3.5a. There is some friction in the joints, but it is very low: Figure 3.4 shows the hopper completely clears the ground once or twice, without any energy injection. This can only mean that the problem is in the drivechain: the low transmission ratio results in a very high motor torque, thereby operating the motor in a very inefficient region. Increasing the transmission ratio would increase the efficiency of the motor, but is

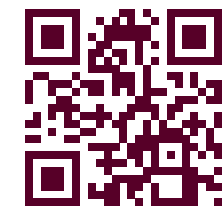

Simulation of hopping

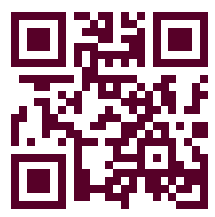

Hopping experiment 


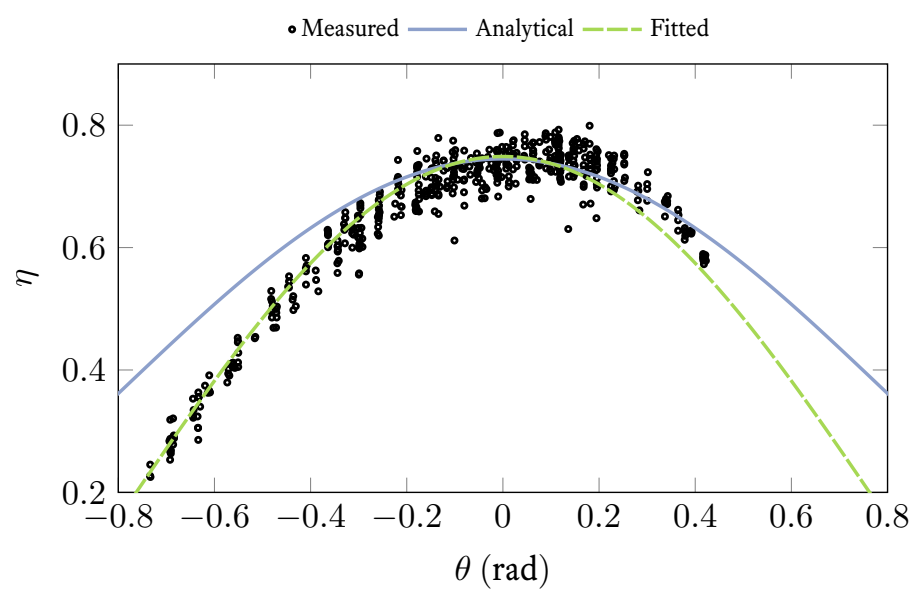

(a) Calculated impact efficiencies from the analytical model, as measured and a fit through the points.

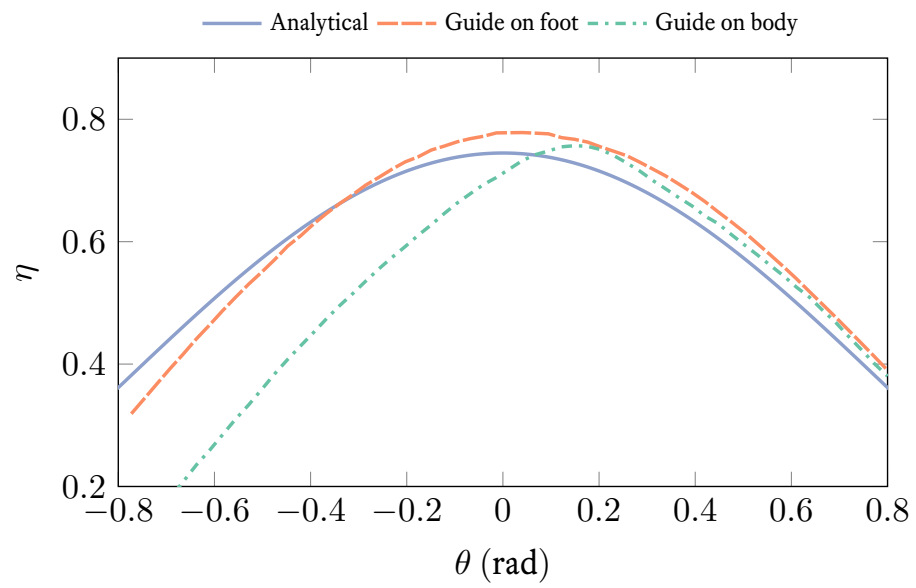

(b) Impact efficiency as found with simulation, compared with analytical results. Simulations performed with the linear guide attached to either the body element or the foot element.

Figure 3.5 - Impact efficiency calculations, measurements and simulation results. The impact efficiency is defined as $\eta=E_{\mathrm{k}}^{+} / E_{\mathrm{k}}^{-}$. 


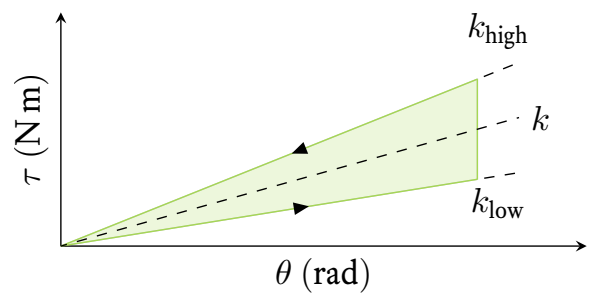

Figure 3.6 - Diagram of the virtual spring created inside the controller. If the leg starts compressing at $\theta=0$, the stiffness is low $\left(k_{\text {low }}\right)$ up to the point of maximum compression. At this point the controller increases the virtual spring stiffness to $k_{\text {high }}$ and the leg starts extending back to the origin.
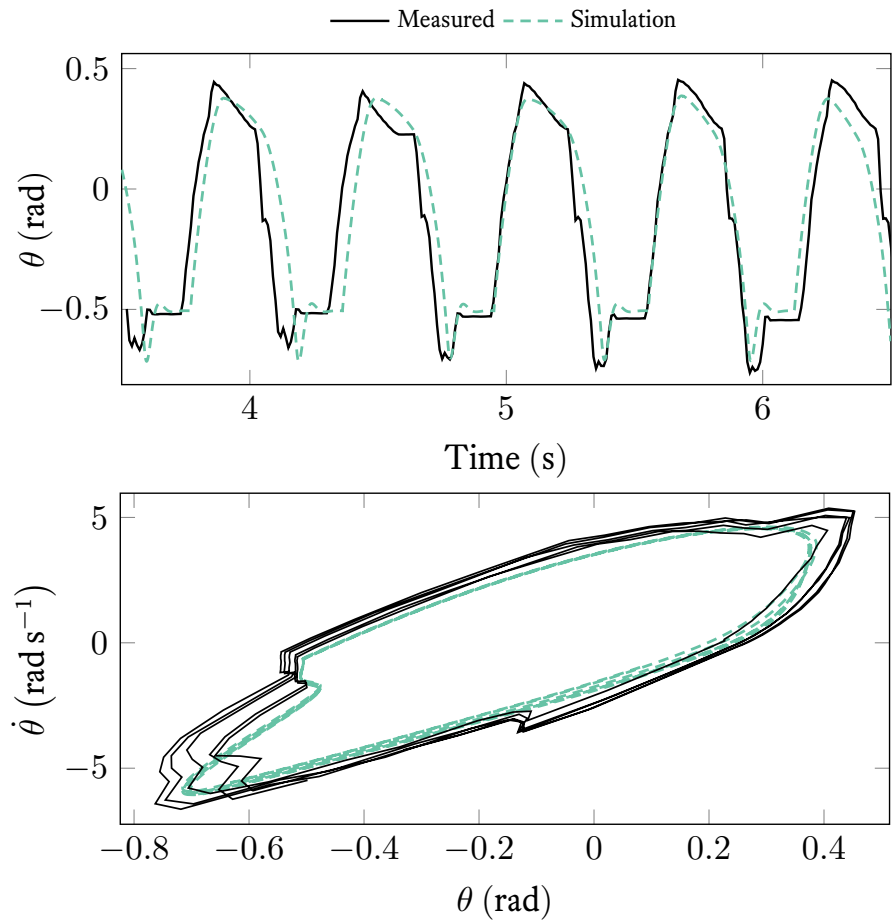

Figure 3.7 - Comparison between the simulated hopping and measurements of the hopping prototype. At the top: leg angle $\theta$ against time, at the bottom the phaseplot of $\theta$. The mechanism is clear of the ground around $\theta=-0.5$, where the leg reaches its maximum extension before the motor is used to hold the leg at $\theta=-0.5$. 


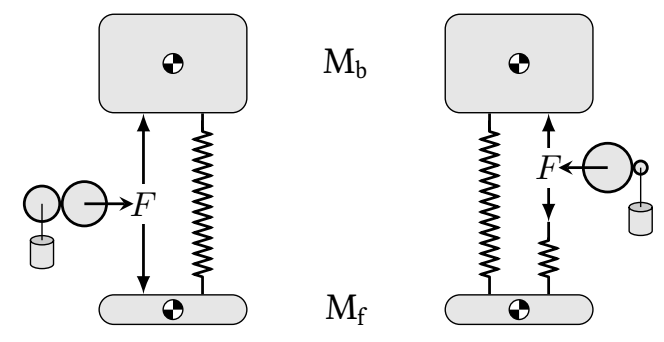

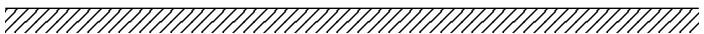

Figure 3.8 - Left: the current prototype. With a relatively low transmission to allow backdrivability the motor has to rotate slowly and is far from its optimal operating speed. Right: proposed new design with series elastics. A higher transmission is possible, resulting in higher motor speeds and the backdrivability is maintained by the spring in series with the transmission.

detrimental to the backdrivability of the mechanism and results in higher impact losses. Instead, we propose a new design where the drive-chain includes a series elastics element, allowing a high transmission ratio while retaining backdrivability. Figure 3.8 illustrates this concept.

\section{$\S 5$ Conclusion}

We have designed and constructed a simple and affordable leg mechanism for resonance-based locomotion. A mathematical description of this mechanism was derived, ultimately leading to an expression for the theoretical impact efficiency. The proposed mechanism was built and used to compare this analytical expression to the real world. The measured results were found to correspond well with the theoretical expectations.

The mechanism was also shown to be capable of hopping. However, the low motor speed and consequently high torque requirements result in the motor heating up. This problem cannot be fixed by increasing the transmission ratio, as this affects the backdrivability of the drive-chain. We intend to solve this problem with a combination of a series elastics drive-chain and parallel stiffness.

With this work, we have gained more insight into the impact loss of mechanisms for legged locomotion. Currently we are developing an improved prototype for use in a combination of such hoppers, which will be used in experiments that aim to realise graceful, resonance-based locomotion. 


\section{Chapter 4}

\section{Spine}

In high-speed locomotion, control is best shared between "brain" and "body": if the natural body dynamics already exhibit desired behaviour, control action can be restricted to stabilising this behaviour, or providing energy to keep it going. This morphological computation can be modelled and designed using Port-Hamiltonian systems (PHS) theory, since the basis of both is the interconnection of dynamic elements. In this chapter, we explore the application of PHS to morphological computation, showing that a three degrees-of-freedom elastic spring functioning as spine in a quadrupedal robot can lead to forward locomotion-without any complicated control action whatsoever.

This chapter was published in Folkertsma, Schaft and Stramigioli [46]. 


\section{§1 Introduction}

Usually, control in robotics focuses on measuring the system state and providing a feedback through actuators; stabilising at a position, following a desired trajectory or realising a controlled impedance. For some years now however, a new field of embodied artificial intelligence or morphological computation has begun to develop, wherein it is recognised that the morphology of the robot itself has a large influence on the behaviour; and that control of the robot could-or rather, should - be shared between "traditional" control and the morphology of the robot ([104]). Good examples of research into this intelligence embodiment include the robots "Puppy" of [72] and "Scout" of [108], both bounding quadrupeds with active hip joints and passive sping-like legs; the "Salamander", a undulatory walking and swimming robot in [73]; and the kangaroo-like resonance-based robots of [85].

The aim in our project is to design a quadruped robot, a "cheetah", exhibiting very fast locomotion with a low energy cost. We believe that in order to achieve this, the desired behaviour (bounding or galloping) has to be at least partly present in the natural dynamics of the system. An energy-based modelling approach is a natural choice when studying energy-efficient locomotion. Port-Hamiltonian Systems (PHS) theory should be very suited for studying morphological computation, considering the similarity between them: exploiting natural body dynamics means obtaining desired behaviour by choosing a proper morphology, in other words the interconnection of e.g. masses and springs; PHS theory explicitly expresses, in an energy-consistent way, the interconnection of various energy storage elements, such as masses and springs, mathematically represented by a Dirac structure $([26,123])$.

In this chapter, we investigate the effect of an elastic spine on quadrupedal running. It is shown that an otherwise completely symmetrical robot model exhibits desired behaviour-forward locomotion - through an asymmetry introduced by an elastic spine. Furthermore, we show that Port-Hamiltonian systems theory, especially in the form of geometrical bond graph modelling, is an excellent way of investigating and designing highly dynamical systems with embodied intelligence.

It has already been shown in multiple quadrupedal runners that an elastic rotational joint in the body or an actuated rotational spine can improve performance: see for instance [33, 24, 109, 61]. However, in this case the spine is a full-dimensional spring with both rotational and translational compliance-moreover, it is solely responsible for any forward locomotion, being the only asymmetry in the model.

Note that, in the final system, control will be shared between "body" and "brain", where the body - through morphological computationcrudely generates the desired locomotion behaviour, while a separate 
controller, the "brain", will be used to stabilise the gait. This chapter addresses the first part, the high-speed morphological computation.

\section{§1.1 Geometrical bond-graph modelling}

Bond graphs are a graphical, energy-consistent modelling language, where storage elements, frictional elements and energy sources are interconnected by bonds that describe power flow between those elements, in the form of effort and flow: generalised force and velocity. The bonds can go through a transformer or gyrator to interface different physical domains or to model power-continuous transformations. In geometrical modelling*, the effort is a wrench and the flow is a twist, both of which have a geometrical interpretation of a screw ([141]). Geometrical reasoning gives direct insight in how to model motions, coordinate transformations and, as is shown later in Section 4.2.1: Spine, a three-dimensional spring $([42,141])$.

Straight running happens mainly in the sagittal plane, so considering the large number of parameters associated with the elastic spine, the quadrupedal robot is modelled in the sagittal plane only. Coordinate frames are indicated by $\Psi_{i}, \Psi_{j}$ with a coordinate change from frame $i$ to $j$ represented by a $3 \times 3$ homogeneous matrix $H_{i}^{j} \in S E(2)$. The velocity of a body $A$ with respect to body $P$, expressed in $\Psi_{k}$ can be expressed as a twist ${ }^{k} T_{A}^{P} \in \mathfrak{s e}(2)$. Wrenches are elements of $\mathfrak{s e}^{*}(2)$; ${ }^{k} W_{A}$ is a wrench exerted on body $A$, expressed in $\Psi_{k}$.

Coordinate transformations of twists and wrenches are calculated by the Adjoint map ([39]):

$$
{ }^{j} T_{A}^{P}=A d_{H_{i}^{j}}^{i} T_{A}^{P} ;{ }^{j} W_{A}=A d_{H_{j}^{i}}^{\top} W_{A}
$$

\section{$\S 2$ Model}

As explained in the introduction, the quadruped robot is modelled in the sagittal plane and as such is two-dimensional. It consists of interconnected rigid bodies and is fore-aft symmetrical, with exception of the spine.

\section{Bodies}

Both the anterior and posterior part consist of three rigid bodies: the body, the leg and the foot, respectively indicated with "body A/P", " $1_{A / P}$ " and " $\mathrm{f}_{A / P}$ " in Figure 4.1. Dimensions and inertial properties of these bodies can be found in Table 4.1 and were chosen to result in a realistic robot model. 


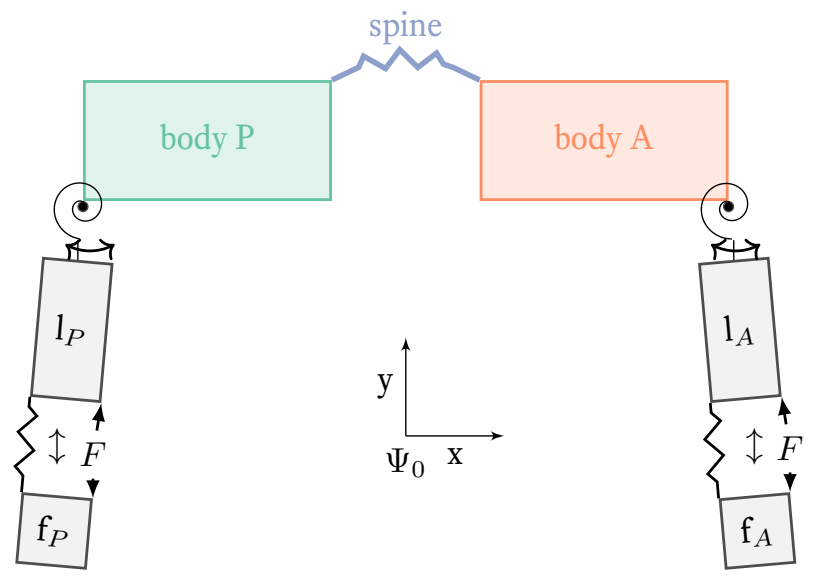

Figure 4.1 - The two-dimensional quadruped robot model. All joints are equipped with passive, linear springs; the ankle joints also feature a force actuator.

Table 4.1 - Body dimensions and inertial properties.

\begin{tabular}{l|rrrr}
\hline & Width & Height & Mass & Inertia \\
\hline Body & $0.5 \mathrm{~m}$ & $0.2 \mathrm{~m}$ & $1 \mathrm{~kg}$ & $0.02 \mathrm{~kg} \mathrm{~m}^{2}$ \\
Leg & $0.5 \mathrm{~m}$ & $0.1 \mathrm{~m}$ & $0.5 \mathrm{~kg}$ & $0.02 \mathrm{~kg} \mathrm{~m}^{2}$ \\
Foot & $0.01 \mathrm{~m}$ & $0.01 \mathrm{~m}$ & $0.05 \mathrm{~kg}$ & $0.001 \mathrm{~kg} \mathrm{~m}^{2}$ \\
\hline
\end{tabular}




\section{Springs}

Stiffness parameters for the hip $\left(K_{h}\right)$ and ankle $\left(K_{a}\right)$ joints are listed in Table 4.2 and were chosen as follows. Firstly, the ankle springs should carry the full body weight without having the feet hit the legs, whilst facilitating bouncing and a stance time that is long enough to allow the controller (see Section 4.2.2: Control) to insert a sufficient amount of energy. A maximum deflection of $25 \mathrm{~cm}$ provides enough stance time. [25] found that during running, the ground reaction force is typically $3-5$ times the body weight, so a stiffness of $300 \mathrm{~N} \mathrm{~m}^{-1}$ allows for $25 \mathrm{~cm}$ of spring deflection.

The hip spring stiffness was chosen such that it allows large deflection during running $\left( \pm 45^{\circ}\right)$ but does not collapse under the body weight. For anteroposterior stability during running, the rest configuration points the legs slightly outward $\left(5^{\circ}\right)$ for a wider stance.

Table 4.2 - Stiffness parameters. Rest configuration is the distance between the foot and the bottom of the leg for ankle springs; the outward rotation (extension) for hip springs.

\begin{tabular}{lrr}
\hline & Hip spring & Ankle spring \\
\hline Stiffness & $33 \mathrm{~N} \mathrm{~m} \mathrm{rad}^{-1}$ & $300 \mathrm{~N} \mathrm{~m}^{-1}$ \\
Rest configuration & $5^{\circ}$ & $25 \mathrm{~cm}$ \\
\hline
\end{tabular}

\section{$\S 2.1$ Spine}

The spine is modelled as a geometric spring parameterised by a centre of compliance, where the spring locally behaves as a decoupled rotational stiffness $k_{z}$ and translational stiffness $K_{t}=\left(\begin{array}{cc}k_{x} & 0 \\ 0 & k_{y}\end{array}\right)$. The elastic wrench is applied in frames $\Psi_{i}$ and $\Psi_{j}$ that are connected to body $A$ and $B$ respectively; the minimal-energy configuration i.e. equilibrium position is when $\Psi_{i}$ and $\Psi_{j}$ coincide and thus $H_{i}^{j}$,eq $=I_{3}$. See Figure 4.2 for a schematic drawing.

For the three-dimensional case an expression for the elastic wrench is known to be ([141]):

$$
\begin{aligned}
{ }^{i} W & =\left(m^{i} f^{i}\right) \\
\tilde{m}^{i} & =-2 \operatorname{as}\left(G_{o} R_{i}^{j}\right)-\operatorname{as}\left(G_{t} R_{j}^{i j} \tilde{p}_{i}{ }^{j} \tilde{p}_{i} R_{i}^{j}\right)-2 \operatorname{as}\left(G_{c}{ }^{j} \tilde{p}_{i} R_{i}^{j}\right) \\
\tilde{f}^{i} & =-R_{j}^{i} \operatorname{as}\left(G_{t}{ }^{j} \tilde{p}_{i}\right) R_{i}^{j}-\operatorname{as}\left(G_{t} R_{j}^{i} \tilde{p}_{i} R_{i}^{j}\right)-2 \operatorname{as}\left(G_{c} R_{i}^{j}\right),
\end{aligned}
$$




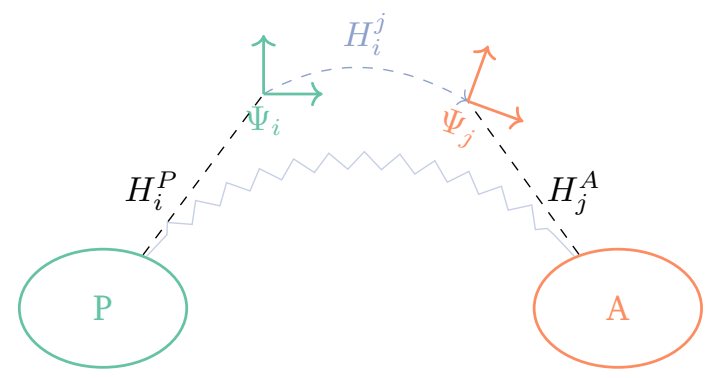

Figure 4.2 - A 3-DoF spring between front and rear body forms the spine.

The elastic wrench generated by this spring is given in (4.3)

where $H_{i}^{j}=\left(\begin{array}{cc}R_{i}^{j} & { }^{j} p_{i} \\ 0 & 1\end{array}\right) \in S E(3)$ and $G_{\{o, t, c\}}$ are the co-stiffness matrices for orientational, translational and coupling stiffness, respectively. as $(M)$ is an operator that returns the antisymmetric part of a matrix $M: \frac{1}{2}\left(M-M^{T}\right)$. The tilde form $\tilde{v}$ is a $3 \times 3$ skew-symmetric matrix for which holds $\tilde{v} w=v \times w$, if $v$ and $w$ are three-dimensional vectors.

In two dimensions, (4.2) reduces to a 3 -DoF elastic wrench:

$$
\begin{aligned}
{ }^{i} W & =\left(m_{z}^{i} f^{i}\right) \\
\left(\begin{array}{cc}
0 & -m_{z}^{i} \\
m_{z}^{i} & 0
\end{array}\right) & =-2 \operatorname{as}\left(\mathbf{G}_{o} R_{i}^{j}\right)-\operatorname{as}\left(\mathbf{G}_{t} w w^{\top}\right) \\
f^{i} & =-\frac{1}{2} R_{j}^{i} \mathbf{K}_{t}{ }^{j} p_{i}-\frac{1}{2} \mathbf{K}_{t} R_{j}^{i j} p_{i}
\end{aligned}
$$

where $w:=R_{j}^{i}\left(\begin{array}{cc}0 & -1 \\ 1 & 0\end{array}\right){ }^{j} p_{i}$ is a vector that is used to calculate the moment resulting from the translational forces of an anisotropic spring. The coupling term $G_{c}$ drops out because it models a coupling between translation along an axis and the torque around that axis and vice versaa screw motion - which is impossible in 2-D.

Of course, $H_{i}^{j}=\left(\begin{array}{cc}R_{i}^{j} & { }^{j} p_{i} \\ 0 & 1\end{array}\right) \in S E(\mathbf{2})$ in this case and the co-

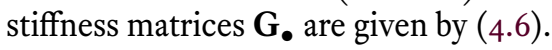

$$
\mathbf{G}_{t}=\frac{1}{2} \operatorname{tr}\left(\mathbf{K}_{t}\right) \mathbf{I}_{2}-\mathbf{K}_{t} ; \quad \mathbf{G}_{o}=\frac{1}{2} k_{z} \mathbf{I}_{2}
$$

The ${ }^{i} W$ of (4.3) is the wrench applied on frame $i$ (which is connected to body A) expressed in the same frame; to obtain the wrench exerted on body B it has to be transformed using the Adjoint of $H_{j}^{i}$ :

$$
{ }^{j} W=A d_{H_{j}^{i}}^{\top i} W .
$$


Note that the Centre of Compliance is characterised by $H_{i}^{P}$ and $H_{j}^{A}$ : when $H_{i}^{j}=0$, frames $\Psi_{i}$ and $\Psi_{j}$ coincide with the CoC.

\section{$\S 2.2$ Control}

We want any locomotion to be the sole result of inherent body dynamics, so the controller should not aim for forward motion but merely excite the system enough to facilitate spontaneous locomotion. The controller therefore only tries to keep the total system energy constant to compensate for friction and impact losses. In order to be able to implement the controller also on a real system, it will not have access to full state information; therefore an estimate for total system energy has to be used. We choose a collocated control architecture where the only sensor is located on the ankle joint and measures displacement $q$ and velocity $v=\dot{q}$. Combined with the actuator force, this results in a proper power port.

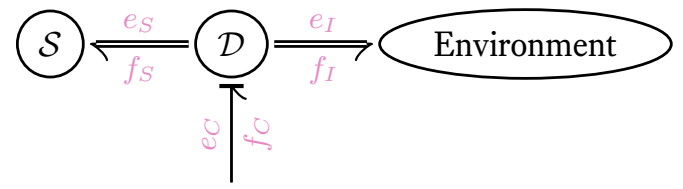

Energy controller

Figure 4.3 - The system in a general PHS view: the controller injects or extracts energy through the control port, aiming to keep the total system energy in $\mathcal{S}$ constant. This energy is estimated once every stride, solely using information available from the control port.

The controller tracks the maximum ankle spring compression during stance $q_{\max }$, which is a measure for the energy stored in the spring andconsidering the vertical speed is zero at this instant-also a measure for the total system energy. This leads to an energy error:

$$
\tilde{E}=E_{\max }-E^{*}=\frac{1}{2} K_{a}\left(q_{\max }\right)^{2}-\frac{1}{2} K_{a}\left(q_{\max }^{*}\right)^{2}
$$

where $E^{*}$ and $q_{\max }^{*}$ are the desired energy and spring compression. This energy $\tilde{E}$ is the surplus (when positive) or shortage (when negative) of system energy, so the actuators have to remove or inject this energy, respectively. While the foot is on the ground, the ankle force actuator can insert or extract energy from the system during a time $\tau_{\text {ground }}$. The power that the actuator must supply during stance is therefore given by 
(4.9), where $\tau_{\text {ground }}$ is the stance time.

$$
P_{c}=\frac{-\tilde{E}}{\tau_{\text {ground }}}
$$

Since $P=\langle e, f\rangle=F^{\top} v$, the force for the actuator is found through (4.10). Infinite control effort is prevented when $v \approx 0$.

$$
F_{c}= \begin{cases}\frac{P_{c}}{v} & |v|>\epsilon \\ 0 & |v|<\epsilon\end{cases}
$$

Figure 4.4 shows a block diagram of this energy controller. It should be noted that $q_{\max }$ can only be determined once per stride. Therefore, the energy error is fed through a PI controller that updates once per stride. The force on the other hand is updated constantly throughout the stride according to (4.10) and set to 0 if the foot is not on the ground.

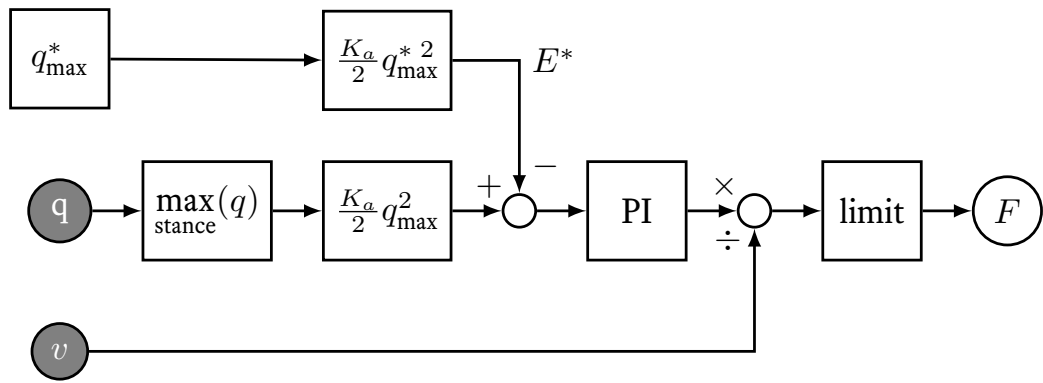

Figure 4.4 - Block diagram of the energy controller; inputs $q$ and $v$ are the ankle joint position and speed; output $F$ is the ankle actuator force. 


\section{§3 Experiments}

The robot model is put through a number of simulation experiments, to investigate the existence of morphological computation and to optimise the performance of the robot. In this section the in silico experimental set-up is explained first; then simulation results are presented.

\section{$\S 3.1$ Simulation}

The bond-graph model of the robot is simulated using the program 20-sim, a multi-domain numerical simulation package by [29]. The ground contact model used is a visco-elastic Hunt-Crossley model in the vertical direction and a Coulomb friction model for the horizontal forces, according to [52]. Figure 4.5 shows a visualisation of the robot model.
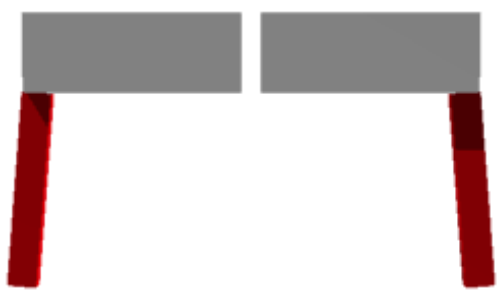

Figure 4.5 - Visualisation of the robot model in the simulation program.

\section{Controller validation}

Firstly, a short experiment was carried out to validate the functioning of the energy controller. During one of the running experiments, the true total system energy* was calculated using the complete system state. Figure 4.6 shows this energy and energy levels calculated in the controller

* Consisting of kinetic energy for the bodies and potential energy for bodies and springs. for one leg. The lower plot show $P_{c}$ and the actually injected power, $P_{\text {actual }}=F^{\top} v$-the dip at mid-stance is caused by the limited control force from (4.10)

The graphs show that $E_{\max }$ is indeed a good estimate of half the total 
system energy, i.e. the energy in one anterior or posterior part. Also, the proposed controller is capable of maintaining a constant energy level and can cope with setpoint changes, if necessary. Notice that the calculated energy level $E_{\max }$ always lags behind 1 stride, because the maximum spring depression is only known after the ground contact phase has ended. Also, the controller can only inject energy when there is ground contact.

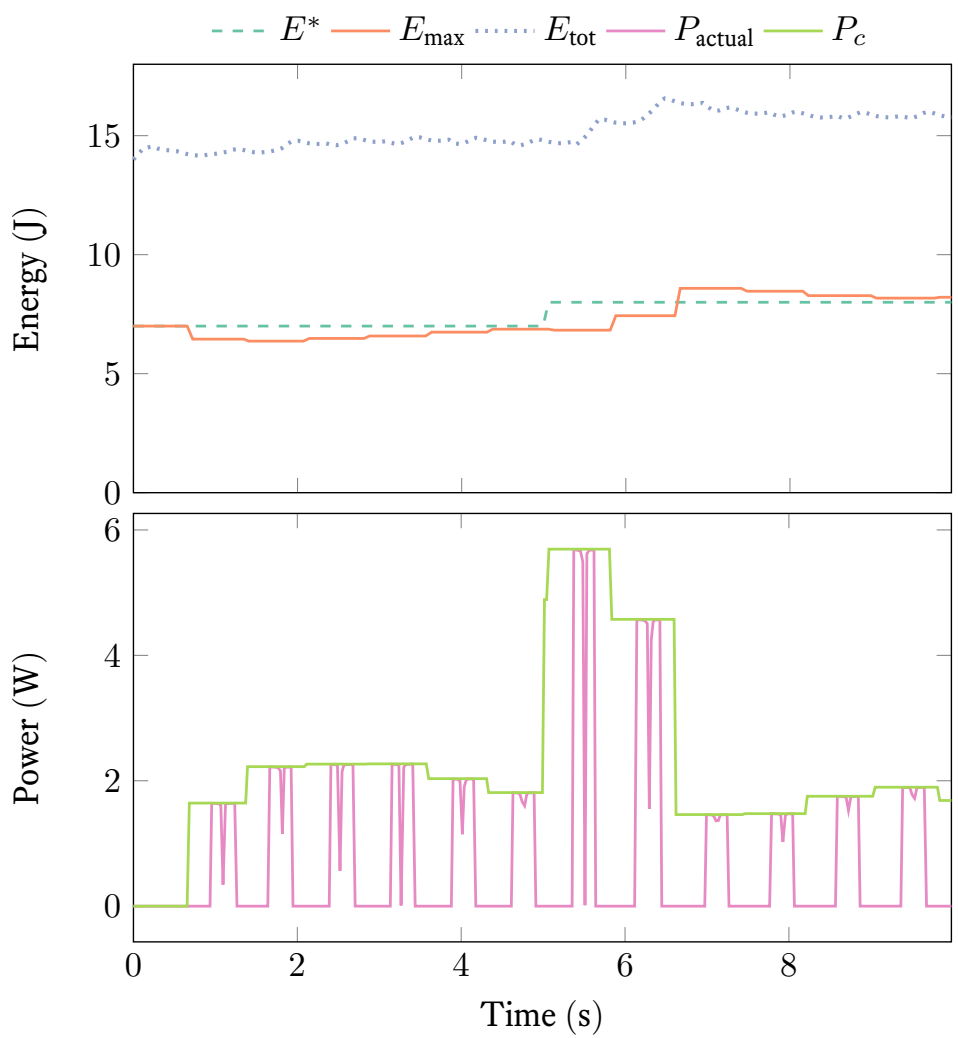

Figure 4.6 - Validation of the energy controller. The top graph shows the energy "setpoint" $E^{*}$, the maximum ankle spring energy after each stride $E_{\max }$ and $E_{\text {tot }}$, the actual total system energy based on the complete model state. The bottom graph shows control power setpoint $P_{c}$ and actually injected power $P_{\text {actual }}$.

\section{Experiment description}

The goal of the simulation experiments is to find morphologies for which the natural body dynamics result in forward locomotion. The model 
is completely symmetrical except for an asymmetry introduced by the spine, the 3 -DoF elastic wrench of (4.3). The three stiffness parameters $k_{z}, k_{x}$ and $k_{y}$ together with a 2-dimensional Centre of Compliance location result in a rather large parameter space to search. However, because the model is simulated in the sagittal plane, multiple simulations can be carried out relatively quickly.

The strategy is therefore to start with a course parameter grid, searching these five parameters in five steps over a reasonable range as shown in Table 4.3. From this initial search, promising regions in this 5-dimensional space are selected for finer searching and optimisation.

Each experiment is identical, save for parameter changes: the robot starts at $x=0$ with the feet $25 \mathrm{~cm}$ above the ground, with zero initial velocity. The performance is the horizontal distance covered in $6 \mathrm{~s}$; an experiment is considered a failure (performance $:=0$ ) if the robot falls, defined when any body's centre of mass goes below $y=0 \mathrm{~m}$.

Table 4.3 - Parameter range for initial search; the parameters are varied linearly over this range in 5 and later 20 steps. CoC location $(0,0)$ corresponds to the frontal end of the posterior body.

\begin{tabular}{r|ccccc}
\hline $\begin{array}{r}\text { parameter } \\
\text { unit }\end{array}$ & $\begin{array}{c}k_{z} \\
\mathrm{~N} \mathrm{~m} \mathrm{rad}^{-1}\end{array}$ & $\begin{array}{c}k_{x} \\
\mathrm{~N} \mathrm{~m}^{-1}\end{array}$ & $\begin{array}{c}k_{y} \\
\mathrm{~N} \mathrm{~m}^{-1}\end{array}$ & $\begin{array}{c}\mathrm{CoC}_{x} \\
\mathrm{~m}\end{array}$ & $\begin{array}{c}\mathrm{CoC}_{y} \\
\mathrm{~m}\end{array}$ \\
\hline minimum & 15 & 50 & 100 & -0.5 & -0.5 \\
maximum & 35 & 150 & 300 & 0 & 0.5 \\
\hline
\end{tabular}




\section{$\S 3.2$ Results}

The results of the coarse parameter search are presented in Figure 4.7, where the averaged performance is shown for each parameter: for each parameter value, the top $40 \%$ of the runs where the parameter had that value is averaged, to convert the $\left(5^{+1}\right)$-dimensional data into a readable graphic. One obvious finding but good to verify nonetheless is that the performance is 0 for $\mathrm{CoC}_{x}=0 \mathrm{~m}$, because this introduces no asymmetry at all.

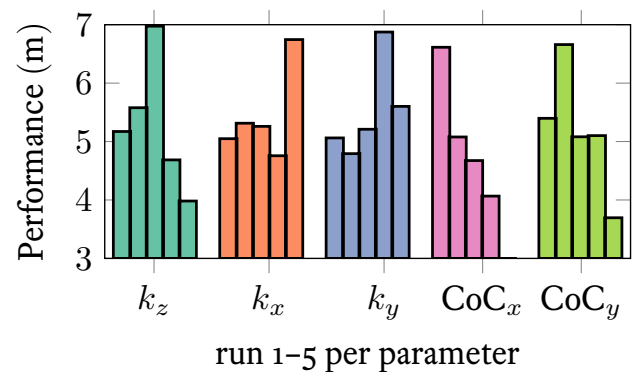

Figure 4.7 - Averaged performance of the top $40 \%$ runs for indicated parameters. Each bar corresponds to the trials where the corresponding parameter is varied according to Table 4.3. The performance for the final run of $\mathrm{CoC}_{x}$ was $1.8 \mathrm{~m}$ and does not fit in the graph.

Based on this first search, the stiffness parameters were frozen to do a fine search and optimisation for the location of the $\mathrm{CoC}$, because the latter has the most influence on asymmetry and locomotion shape. The values chosen are $k_{z}=25 \mathrm{Nm} \mathrm{rad}^{-1} ; k_{x}=150 \mathrm{~N} \mathrm{~m}^{-1} ; k_{y}=$ $250 \mathrm{~N} \mathrm{~m}^{-1}$. The result of this search is shown in Figure 4.8 with the performance, i.e. distance covered in $6 \mathrm{~s}$ on the vertical axis.

This $3-\mathrm{D}$ plot is a bit hard to read, so instead it is interesting to see whether there are regions for the $\mathrm{CoC}$ where the robot shows good performance, i.e. the peaks of the graph in Figure 4.8. Figure 4.9 shows those $\mathrm{CoC}$ locations where the performance was at least $60 \%$ of the maximum performance.

It is clear that there is a large region where the asymmetry introduced by the spine results in consistent forward locomotion: if the Centre of Compliance lies somewhat below the posterior body's centre of mass, the robot model accelerates from a complete standstill to a speed of over $2 \mathrm{~m} \mathrm{~s}^{-1}$. There is also a second, smaller region above the posterior body that leads to forward locomotion: the gait there is different, and the distance covered less. Figure 4.10 shows frames of a visualisation of the running robot model. 


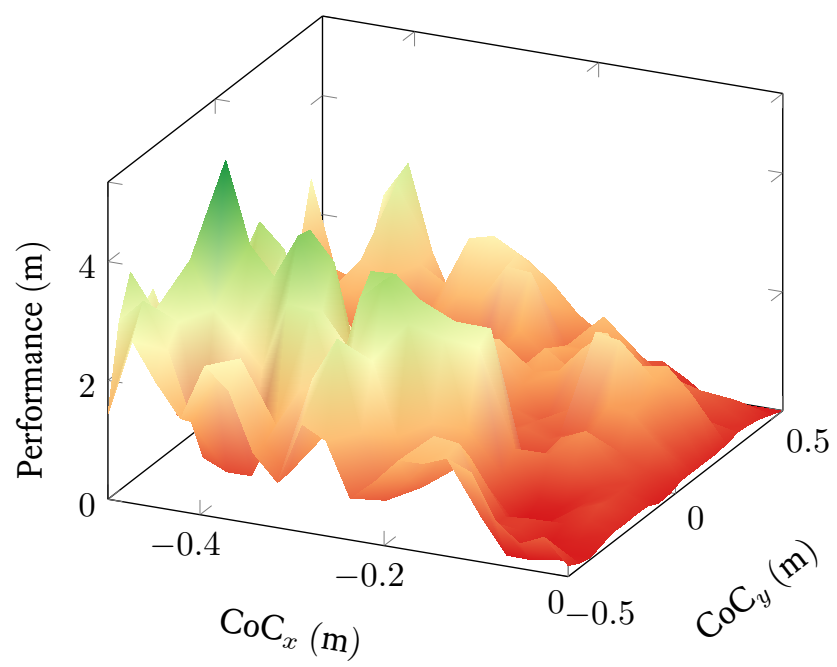

Figure 4.8 - Fine CoC location search (20 steps) with fixed stiffness parameters.

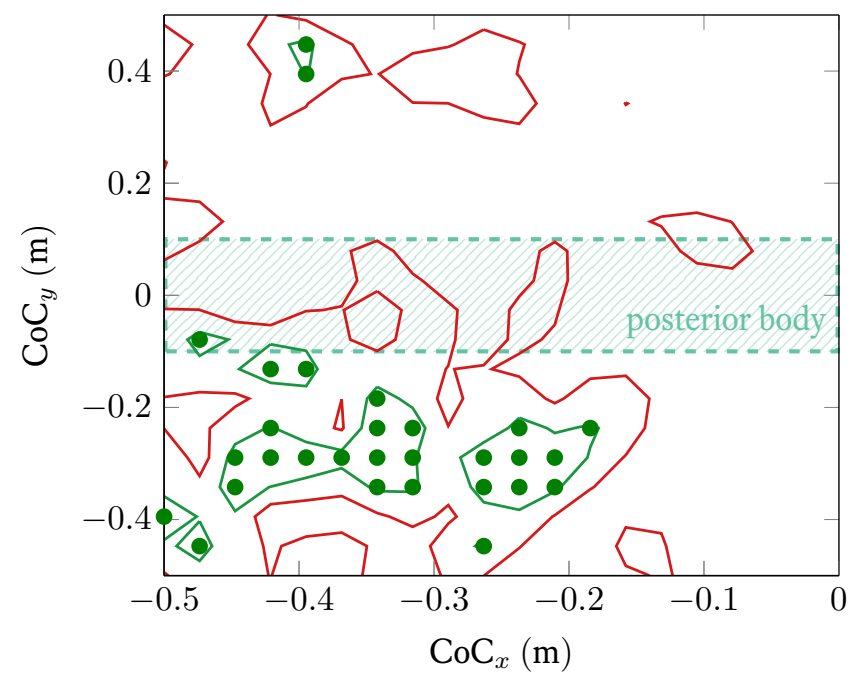

Figure 4.9 - Thresholded performance of Figure 4.8, showing only those $\mathrm{CoC}$ locations where the covered distance was at least $60 \%$ of the maximum. Contour lines are shown for 40 and $60 \%$. 


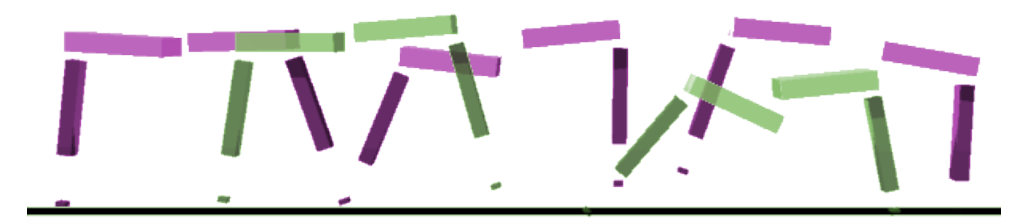

Figure 4.10 - Stills from a visualisation of the robot, showing a gait cycle typical for the high-performance $\mathrm{CoC}$ region.

\section{$\S 4$ Conclusion}

\section{$\S 4.1$ Conclusions}

In this chapter, we have shown a way to model and parametrise an elastic spine; and that this spine can lead to forward locomotion. In an otherwise completely symmetrical model, the morphological computation introduced by the spine led to natural body dynamics resulting in forward locomotion. The energy-based controller injected energy into the system to keep the total constant, compensating for friction and impact losses and otherwise not pushing towards a certain gait.

Furthermore, we have successfully used the Port-Hamiltonian Systems theory in combination with geometrical bond graph modelling for design and analysis of the robot model, opening the way for more high-speed morphological computation analyses and designs.

\section{$\S 4.2$ Future work}

In the future, we want to take the approach with geometric spring further: we are most interested in using this framework to investigate morphosis, i.e. the active changing of morphology. It is theoretically possible to change the Centre of Compliance on a running system, by adding two power ports to the elastic element (see Figure 4.11 for a conceptual drawing). In this way, $H_{i}^{A}$ and $H_{j}^{B}$ can be influenced-what's more, by choosing a proper interconnection for these ports it is possible to do so in a power-continuous way. Hence, it is possible to control the elastic wrench without using any energy. If the elastic forces can be shaped so as to generate high-speed locomotion, high performance can be obtainedwithout using any additional energy!

On the theoretical/simulation side, we are looking at ways to improve this locomotion by designing a more intelligent controller that aims to turn this "crude" system behaviour, induced by natural body dynamics, into a properly stabilised limit cycle. 


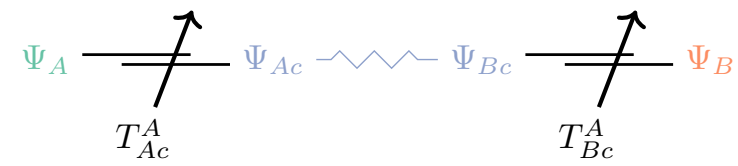

Figure 4.11 - A conceptual drawing of a spring with changeable $\mathrm{CoC}$ through two additional power ports.

At the same time, we are working on a prototype sagittal-plane runner that can demonstrate the practical feasibility and - hopefully-effectiveness of the elastic spine. The first step, literally, was to design a one-legged hopper that is suitable for resonance-based locomotion [163]. Current research focuses on practical implementations of a spring with adjustable centre of compliance. 
Chapter 4 


\section{Chapter 5}

\section{Synchronisation}

Synchronisation is an important phenomenon when studying multiple dynamic systems: it describes whether the systems "move in lock-step." Our motivation to study synchronisation stems from legged locomotion: gait patterns describe a desired "phase difference" between the legs. In this paper we give a novel, geometric definition of synchronisation and phase, which can be applied to define and achieve synchronisation of arbitrary limit cycles. From a control-by-interconnection perspective, we aim to create and synchronise limit cycles of dynamic systems in a power-continuous way. We show several examples of power-continuous synchronisation: synchronisation of dynamical systems achieved without using external energy. The new geometric definition is a coordinate-free description of synchronisation that gives insight into the concepts of synchronisation, phase and phase difference, that can be applied to all synchronisation problems. For us, it opens the way to better-performing legged locomotion.

This chapter has been submitted to the International Journal of Robust and Nonlinear Control in January 2017. 


\section{§1 Introduction}

Synchronisation is "The operation or activity of two or more things at the same time or rate," according to the dictionary ([136]). It is an important phenomenon in the behaviour of multiple dynamic systems: when systems act in concert, they are synchronised. Or, when they have to act in concert, they must be synchronised.

Synchronisation is studied in many fields: in robotics, where multiple "agents" have to work together (e.g. [115]), or to solve the age-old mystery of Huygens' synchronised pendulum clocks ([66]). In biology, synchronisation is studied both inter- and intra-organism: neurones exhibit synchronisation phenomena, [53]; fireflies blink in a synchronous manner, [89]. The electricity grid consists of multiple generators that have to be synchronised to each other, something that will become a larger challenge with many localised generators (wind turbines, solar panels); see $[14,124,11]$.

\section{$\S 1.1$ Synchronisation in dynamic locomotion}

Our motivation for studying synchronisation stems from (energy-efficient) dynamic locomotion. With dynamic locomotion, we indicate that the feet are not simply position-controlled, like when walking, but that the dynamics of the whole robot play a significant effect in the trajectory of the feet. In locomotion, all limbs have to move in a certain gait pattern, so they have to be synchronised. A running quadruped, for example a cheetah, can be seen as a collection of rigid bodies all executing synchronised limit cycle oscillations: the anterior and posterior body move with the spine; the legs execute a galloping footfall pattern; even the separate parts of each leg are locked in a periodic motion.

Because the system dynamics have such a high influence in dynamic locomotion, the overall goal is to (physically) shape them such that the inherent, natural dynamics exhibit much of the desired dynamics: locomotion. This methodology is called "morphological computation," because the morphology of the robot takes over "computation" from the control algorithms; [104]. The central role of morphology - the interconnection of masses and springs - motivates a modelling and control framework wherein this interconnection is explicit and central: Control by Interconnection; [95]. In this framework, (sub)systems and control algorithms are interconnected by power ports, which has the added benefit of giving direct insight into energy flows in the system. This in turn gives insight into-and possibly control over-energy-efficiency. Using this methodology and framework, in [46] we showed successful morphological computation in the spine of a planar cheetah robot. However, synchronisation between the anterior and posterior leg was an emergent 
behaviour, depending on the initial conditions. Often it was chaotic or unstable: the performance of the quadruped may be greatly improved if there is more control over the synchronisation.

\section{$\S 1.2$ Related work}

There is a large amount of publications on synchronisation phenomena. A well-known synchronisation model is the Kuramoto model ([79]), treated in detail in [31], where the systems-to-be-synchronised are phase oscillators of the form

$$
\dot{\theta}_{i}=\omega_{i}+u,
$$

where $u$ is an input term depending on the coupling of the individual oscillators. Further work on Kuramoto models is presented in [1, 147], with extended results on stability and collective synchronisation. Although the phase oscillator of (5.1) is a simple system, it can be used to describe the "phase behaviour" of many periodic systems, where $\omega$ is each system's oscillator frequency [152].

The phase behaviour is not always an adequate description of the system dynamics, for example because the coupling between the subsystems has a significant effect on their dynamics. In this case, the systems are often identical oscillators that have to become synchronised, i.e. the state or output of each system has to become equal. Some examples are [148] (Lur'e systems), [99, 10o] (chaotic systems) and [121] (linear systems). Since the systems are identical and a large number of systems is considered, the focus in these papers is on the network structure that connects the systems. Each system's (identical) control law, in combination with the interconnection, is used to obtain consensus on state $x$ or output $y$ of each system; [92, 27]. Consensus on $x$ means:

$$
\lim _{t \rightarrow \infty} x_{i}(t)=\bar{x}(t),
$$

i.e. all evolutions of the coupled systems tend to the same solution.

For the Kuramoto model, it is also clear what synchronisation means: the rate of each phase oscillator becomes equal. For the more complicated dynamic models, it is more difficult: what is the phase of a random oscillator or arbitrary limit cycle? If different systems have to be synchronised, or if a phase difference is desired, consensus is an insufficient description of synchronisation. [116] proposed to use the instantaneous amplitude and phase of an arbitrary signal, to define phase and phase difference. The drawback is that the signal analysis has little relation with the actual system and system dynamics. In [15], a very generic and formal definition of synchronisation is presented: functionals on the outputs of the systems, possibly time-shifted, define whether the systems are synchronised. This definition of synchronisation is much closer related 
to the system dynamics, but is still a very coordinate-dependent definition and does not give much geometric insight into the synchronisation phenomenon. Control of oscillating systems is studied in [133] and [132]. Here, synchronisation is regarded as convergence of the trajectories to a diagonal set in the state space of the overall system.

In [47] we showed a method for power-continuous synchronisation of mass-spring oscillators and applied it on two "hoppers" that modelled two legs of a quadruped. In this work, we will extend this work to the power-continuous synchronisation of dynamic systems with arbitrary limit cycles.

\section{$\S 1.3$ Problem formulation}

We will investigate the generation and mutual synchronisation of limit cycle oscillations. Although the application in mind is dynamic locomotion, the approach is more general. First, we aim to define synchronisation of limit cycles in a coordinate-free way (Section 5.2: Synchronisation). Then, we use some example systems to study how limit cycles may be generated and how these limit cycles may be synchronised, both in a power-continuous way (Section 5.3: Van der Pol oscillators and Section 5.4: Circling masses).

As a graphical notation for both the dynamic systems and the Controlby-Interconnection controllers, we use bond graphs $([98,18])$. This notation method has close ties with Port-Hamiltonian Systems theory, [123]. In both frameworks, system dynamics are characterised by energy functions, the Hamiltonian, and interconnection between energy-storage elements. Figure 5.1 shows the problem set-up: two systems, $\Sigma_{1}$ and $\Sigma_{2}$, have some periodic behaviour. This limit cycle behaviour may be created or stabilised by a controller $\mathcal{C}_{i}$. The two systems are interconnected by a synchronisation controller, $\mathcal{C}_{\text {sync }}$. The aim is to:

1. generate or stabilise periodic behaviour of the system $\Sigma_{i}$ by controller $\mathcal{C}_{i}$, if necessary;

2. define a "phase" for this periodic behaviour (limit cycle);

3. synchronise the two systems by regulating the "phase difference."

All of this should be accomplished in a power-continuous way, in other words: the controllers may only modulate the interconnection structure and thereby "direct" the energy flow; not modify the actual energy contents of the system. 


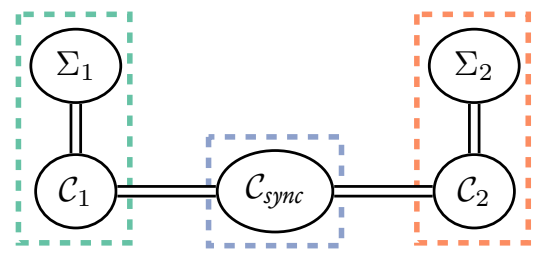

Figure 5.1 - The lay-out of the problem: two systems with periodic behaviour - whether or not caused by a controller $\mathcal{C}_{i}$ - synchronised by a controller $\mathcal{C}_{\text {sync }}$. The double bonds between the subsystems indicate power interconnections and consist of a power-conjugate pair of variables, like force and velocity.

\section{§2 Synchronisation}

The answer to the problem, as is often the case, starts with finding the right question. The answer to the synchronisation problem, therefore, starts with the question: what is synchronisation?

We propose a geometric definition for synchronisation. In the following definition, the dynamic systems are expressed in the Port-Hamiltonian System formulation, but the synchronisation is independent of this formulation.

Definition 1. Given two systems $\Sigma_{i} i=1,2$ as follows:

$$
\begin{aligned}
\dot{x}_{i}(t) & =\left[J_{i}\left(x_{i}\right)-R_{i}\left(x_{i}\right)\right] \frac{\partial H_{i}\left(x_{i}\right)}{\partial x_{i}}+g_{i}(x) u_{i}(t) \\
y_{i} & =g_{i}^{\top}(x) \frac{\partial H_{i}^{\top}\left(x_{i}\right)}{\partial x_{i}}
\end{aligned}
$$

where $x_{i} \in \mathcal{X}_{i}$, some metric space. Take $u_{i}(t) \equiv 0$.

Suppose that the two systems both have at least one stable limit cycle $\ell_{i} \in X_{i}$, a closed trajectory in the state space of system $i$. Due to the topology of $\ell_{i}$ it is possible to consider homeomorphic maps $\Phi_{i}: \ell_{i} \rightarrow$ $\mathcal{S}^{1} ; x_{i} \mapsto s_{i}$, which map a point on the limit cycle to a point on a circle.

Let's indicate with $\Theta: \mathcal{S}^{1} \rightarrow \mathcal{S}^{1}$ a homeomorphic map. We call the systems synchronised for $\Theta$ if the following holds when both systems are in their limit cycle $\ell_{i}$ :

$$
x_{2}(t)=\left(\Phi_{2}^{-1} \circ \Theta \circ \Phi_{1}\right) x_{1}(t) \quad \forall t
$$

The drawing in Figure 5.2 illustrates this definition: $\Phi$ maps each limit cycle to a circle, and between these mapped limit cycles there exists a constant map $\Theta$. It is not necessary to first map $\ell_{i}$ to $\mathcal{S}^{1}$, but doing so makes comparison of different limit cycles easier. 


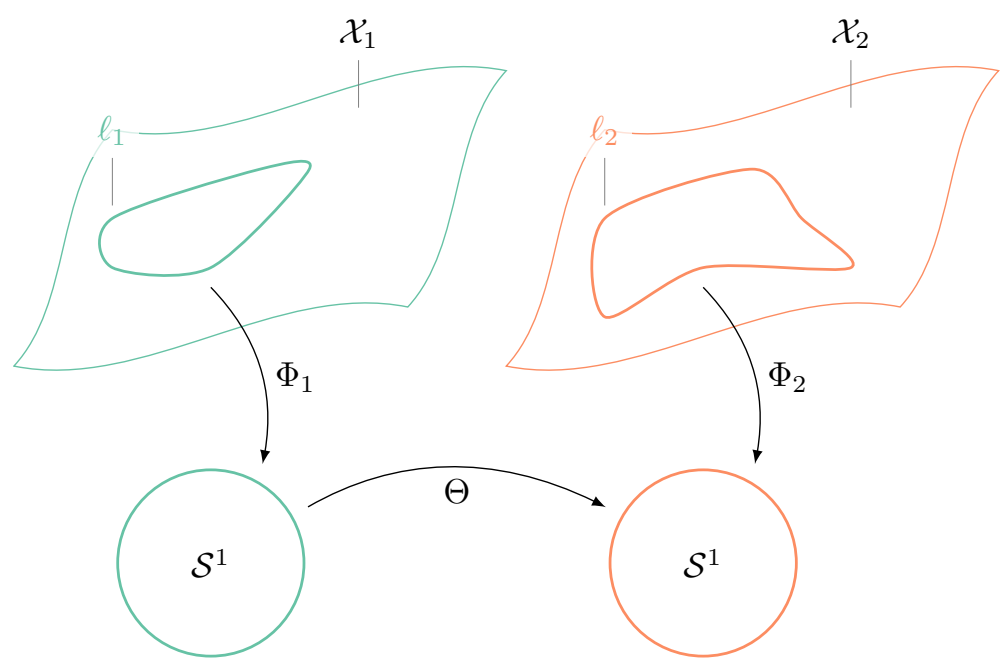

Figure $\mathbf{5 . 2}$ - Two systems are synchronised if there is a homeomorphic map between their periodic trajectories, or-equivalently, but more intuitively-between the mappings of their limit cycles to $\mathcal{S}^{1}$.

Definition 2. Let $\phi_{i}$ be the local coordinate on $\mathcal{S}^{1}$ of the mapped limit cycle, i.e.

$$
\phi_{i}:=\Phi_{i}\left(x_{i}\right) .
$$

$\phi_{i}$ is called the phase of $\Sigma_{i}$. A straightforward coordinate choice for the synchronisation map $\Theta$ is the difference of $\phi_{i}$,

$$
\theta:=\phi_{2}-\phi_{1}
$$

which we call the phase difference.

A possible choice for the parametrisation is normalised time:

$$
\phi_{i}=\frac{t-t_{i}^{0}}{T_{i}}
$$

where $t_{i}^{0}$ is the time when the $x_{i}(t)$ passes a particular point $x_{0}$ on the limit cycle; $T_{i}$ is the period of $\ell_{i}$. Because point $x_{0}$ is passed every cycle, $t_{i}^{0}$ is reset every period and thus $\phi_{i} \in[0,1)$.

Theorem 5.2.1. The systems of Definition 1 can be synchronised under either of the following necessary and sufficient conditions:

a) $T_{1}$ and/or $T_{2}$ can be changed continuously, at least enough to make $T_{1}$ equal to $T_{2}$; 
b) $T_{1}=T_{2}=T$ and the basin of attraction of at least one of the limit cycles allows a temporary deviation that causes a required change $\Delta T$ from the normal period.

Proof. For case $b$, it is clear that the phase difference $\theta$ is constant if both systems are on their limit cycle:

$$
\phi_{2}-\phi_{1}=\frac{t-t_{2}^{0}}{T_{2}}-\frac{t-t_{1}^{0}}{T_{1}}=\frac{t_{1}^{0}-t_{2}^{0}}{T}
$$

Furthermore, a small disturbance to one of the systems that causes a temporary deviation $\Delta T$ from the period $T_{i}$ will change $t_{i}^{0}$. Doing this in such a way that

$$
t_{1}^{0}=t_{2}^{0}+\theta_{s} T
$$

will result in two synchronised limit cycles with desired phase difference $\theta_{s}$ :

$$
\theta=\frac{t_{1}^{0}-t_{2}^{0}}{T}=\frac{t_{2}^{0}+\theta_{s} T-t_{2}^{0}}{T}=\theta_{s} .
$$

For case $a$, the proof is equal to case $b$ when $T_{1}$ and/or $T_{2}$ is changed such that $T_{1}=T_{2}=T$ and $\Delta T$ is a direct result of influencing $T_{1}$ and/or $T_{2}$.

The theorem makes clear that two systems can be synchronised if their period is equal. The phase difference may be controlled if the period of at least one of the systems can be influenced, to control the time delay and thus phase. However, this time-based definition of phase is not very much connected to the system dynamics.

A better definition for the phase would depend on the state $x_{i}$. The problem is that it is often non-trivial to assign a phase $\phi_{i}$ to the current state of $\Sigma_{i}$, especially when (as in case $b$ ) the system is not currently on the limit cycle. However:

Corollary 5.2.2. If a continuous surjective map from $X_{i}$ to $\mathcal{S}^{1}$ is defined, and the restriction of this map to $\ell_{i}$ is homeomorphic to $\mathcal{S}^{1}$, then a synchronising controller $\mathcal{C}_{\text {sync }}$ can be found for systems, as described in Theorem 5.2.1.

\section{$\S 2.1$ Extension to off-limit cycle}

Definition 1 defines synchronisation when the systems are on their limit cycle, i.e. only for $x_{i} \in \ell_{i}$. This is because the limit cycle is topologically equivalent to $\mathcal{S}^{1}$, so the map $\Phi_{i}$ can always be defined. However, when the system is not on its limit cycle (yet), the map $\Phi: \ell \rightarrow \mathcal{S}^{1}$ cannot be used. This is also the case when the system is actively perturbed from the limit cycle to achieve synchronisation, as in Theorem 5.2.1. Especially 
in the latter case, it is essential to define the phase and phase difference also when $x_{i} \notin \ell_{i}$, as proposed in Corollary 5.2.2.

A projection of $\mathcal{X}_{i} \rightarrow \ell_{i}$ would suffice, but this projection cannot necessarily be defined globally as a continuous surjection, depending on the topology of $\mathcal{X}_{i}$. Instead, we assume the system to stay close to the limit cycle ${ }^{1}$. Let $\mathcal{L}_{i}$ be a submanifold of $\mathcal{X}_{i}$ such that $\ell_{i} \in \mathcal{L}_{i}$. Furthermore, $\mathcal{L}_{i}$ is a fibre bundle defined by projection $\pi_{i}: \mathcal{L}_{i} \rightarrow \ell_{i}$, with base space $\ell_{i}$ and fibres $\pi_{i}^{-1}\left(l_{i}\right), l_{i} \in \ell_{i}$. Now we can define a new phase map $\Phi_{i}^{\prime}: \mathcal{L}_{i} \rightarrow \mathcal{S}^{1}$ :

$$
\Phi_{i}^{\prime}:=\Phi_{i} \circ \pi_{i} .
$$

Due to the structure of the fibre bundle, this map is a continuous surjection and thus we can determine phase, phase difference and synchronisation when $x_{i} \in \mathcal{L}_{i}$.

$\mathcal{L}$ defines a "tube" around the limit cycle (see Figure 5.3). The size cross-section of the tube depends on how "close" the system is going to stay to the limit cycle. If $\mathcal{L}$ is in the limit cycle's basin of attraction, then the active synchronisation control of Theorem 5.2.1- $b$ stays within $\mathcal{L}$.

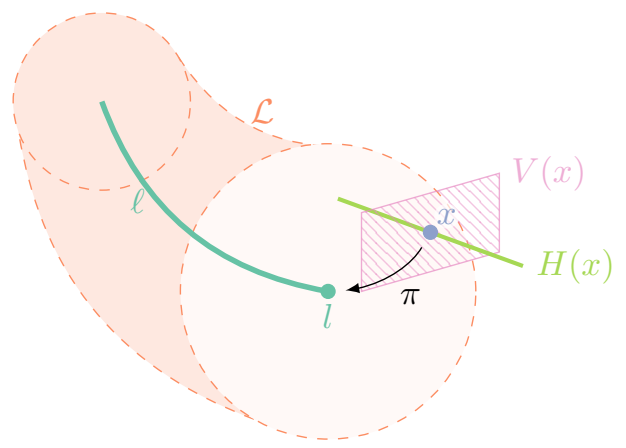

Figure 5.3 - Definition of a "tube" $\mathcal{L}$ around the limit cycle $\ell$ by means of a fibre bundle. Using this structure, the tangent space $T \mathcal{L}$ can be decomposed into a horizontal and vertical part; then, the horizontal part can be mapped to the limit cycle $(T \ell)$ and thus to $T \mathcal{S}^{1}$.

\section{$\S 2.2$ Tangent maps}

A natural phase control method is allowed when there are also tangent maps from the limit cycle and its neighbourhood $\mathcal{L}_{i}$ to $\mathcal{S}^{1}$. For the definition of synchronisation, the maps $\Phi_{i}$ and $\Theta$ must be homeomorphisms.

${ }^{1}$ The metric to define closeness depends on the system in question. In the portHamiltonian formulation, the metric could be defined using the Hamiltonian $\mathcal{H}(x)$ : the boundary of the "tube" can be a "level set" of energy. 
If the maps are diffeomorphisms, then there also exists a natural map for the tangent spaces $T \ell_{i}$. This tangent map must be extended to $\mathcal{L}_{i}$ to allow phase control when $x_{i}$ is not exactly on the limit cycle. If an Ehresmann connection is defined on $\mathcal{L}_{i}$, the tangent space to $\mathcal{L}_{i}$ can be split in horizontal and vertical parts:

$$
T_{x} \mathcal{L}=H(x) \oplus V(x) .
$$

$H(x)$ is the tangent space that is parallel to the limit cycle $\left(T_{x} \ell=\right.$ $H(x), x \in \ell) ; V(x)$ is the vertical tangent space, along the fibres. (See also Figure 5.3) The tangent vector for any point $l_{i} \in \mathcal{L}$ can be projected on the horizontal space. Finally, this horizontal part hor $v_{i}$, of any $v_{i} \in T \mathcal{L}_{i}$, can be mapped to $T \mathcal{S}^{1}$ using the diffeomorphism $\Phi_{i}$. Although the formulation is different, this "tube" and the decomposition into horizontal and vertical, or parallel and transverse parts, is similar to the method of "transverse linearisation" by [134].

The benefit of having these tangent maps is that it allows a natural phase control method as follows. $\mathcal{C}_{\text {sync }}$ connects to the two systems through power ports of power conjugate variables, say velocity $v$ and force $F$. The decomposition of $\mathcal{L}$ into $H(x)$ and $V(x)$ has a dual:

$$
T_{x}^{*} \mathcal{L}=H^{*}(x) \oplus V^{*}(x) ; \quad H^{*}, V^{*} \subseteq T^{*} \mathcal{L} .
$$

$H^{*}$ is taken as $V^{\perp}$ and $V^{*}$ as $H^{\perp}$, the orthogonal complements with respect to the duality product. The power that flows through the power port between $\Sigma_{i}$ and $\mathcal{C}_{\text {sync }}$ can then also be split:

$$
P_{i}=\langle v \mid F\rangle=\left\langle v_{\mathrm{H}} \mid F_{\mathrm{H}}\right\rangle+\left\langle v_{\mathrm{V}} \mid F_{\mathrm{V}}\right\rangle,
$$

where subscripts $\mathrm{H}$ and $\mathrm{V}$ denote the horizontal and vertical parts. Now, the system can be steered towards the limit cycle in the vertical space, using $F_{\mathrm{V}}$; and along the limit cycle-even when it is not currently on the limit cycle-using $F_{\mathrm{H}}$. Clearly, the latter can be used for synchronisation, by "speeding up" or "slowing down" along the limit cycle.

In other words, the map from $T \mathcal{L}_{i} \rightarrow T \mathcal{S}^{1}$ projects the "phase velocity" of system $i$ onto the tangent space of the synchronisation controller, but it also gives rise to a natural adjoint map in the opposite direction, from $T^{*} \mathcal{S}^{1} \rightarrow T^{*} \mathcal{L}_{i}$. That is to say, there is a map for the control action in phase space $\mathcal{S}^{1}$ to the state space $\mathcal{X}_{i}$, or at least to an open subspace $\mathcal{L}_{i}$ around $\ell_{i}$.

This means that a proper definition of the synchronisation with a diffeomorphic map and a fibre bundle immediately leads to a natural control over the phase. In the example in Section 5.4: Circling masses this is employed in finding the (power-continuous) synchronisation controller. 


\section{$\S 2.3$ Power-continuous synchronisation}

From an energy-efficiency or passivity perspective, it is interesting to find power-continuous control algorithms. That is to say, the controller does not influence the energy balance or energy content of the total system, but only modulates the power flows; modulates the interconnection between the subsystems. In the examples hereafter, we will aim for power-continuous synchronisation, defined as follows.

Definition 3. Given the two systems of Definition 1 and a third Port-Hamiltonian System $\mathcal{C}_{\text {sync. }}$. The three passive systems are interconnected through a power-continuous Dirac structure. By the PHS structure, the interconnected system is also a passive Port-Hamiltonian System. If $\Sigma_{1}$ and $\Sigma_{2}$ can be synchronised by $\mathcal{C}_{\text {sync }}$, this requires - by definition - no external energy supply. This is called power-continuous synchronisation.

\section{$\S 2.4$ Examples}

The definitions and extensions given above are geometric and abstract definitions, where the choice for $\Phi, \Theta$ and the synchronisation controller are dependent on the systems-to-be-synchronised. Because of the generic, coordinate-free definitions, this concept of synchronisation can be applied to any kind of dynamical system, also to define or achieve synchronisation between two completely different type of systems. To demonstrate the application of the theory outline above, we show two examples in the following sections.

Van der Pol A Van der Pol oscillator can exhibit a stable limit cycle. In the example in Section 5.3: Van der Pol oscillators, we synchronise two Van der Pol oscillators by modifying their limit cycle period, like proposed in Theorem 5.2.1. Because the Van der Pol oscillator itself is not a passive system, we first find an energy-conservative formulation of the dynamics. Next, a power-continuous synchronisation controller will be derived.

Circling masses Two masses moving in a 2-D plane can be made to follow a circular trajectory: when done in a passive way, this results in a power-continuous limit cycle. Next, the two masses are synchronised, again using a power-continuous synchronisation controller. In this example in Section 5.4: Circling masses, the extension of Section 5.2.2: Tangent maps will be used for both controllers. 


\section{$\S 3$ Van der Pol oscillators}

Van der Pol oscillators are nonlinear oscillators described by the dynamical equations in (5.15).

$$
\ddot{x}-\mu\left(1-x^{2}\right) \dot{x}+x=0 .
$$

For positive values of $\mu$, they exhibit a stable limit cycle and thus we can attempt to synchronise two Van der Pol oscillators. To put them into the Port-Hamiltonian System framework, we recognise that they are equivalent to a mass-spring-damper system with $m=1, K=1$ and nonlinear, state-modulated damper $R=\mu\left(1-x^{2}\right)$ (see Figure 5.4a).

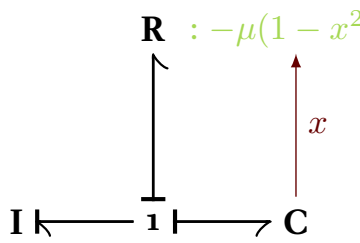

(a) Nonlinear, state-modulated damper $\mathbf{R}$.

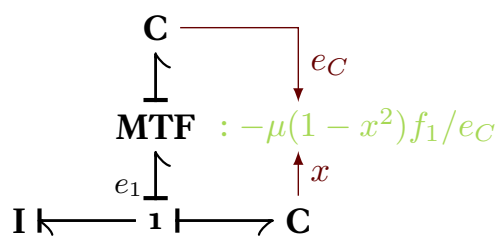

(b) State-and-co-energymodulated MTF and storage element.

Figure 5.4 - Van der Pol oscillator represented as physical system.

\section{§3.1 Energy-conservative Van der Pol}

The energy of the Van der Pol oscillator modelled a a mass-springdamper system can be easily found: it is the sum of the kinetic (co-)energy of the mass and the potential energy of the spring:

$$
E_{\text {vanderpol }}=\frac{1}{2} m \dot{x}^{2}+\frac{1}{2} K x^{2}=\frac{1}{2}\left(\dot{x}^{2}+x^{2}\right) .
$$

This energy is defined by the state $(x, \dot{x})$ and thus it is easy to see that in the limit cycle, the energy over one period is constant:

$\mathbf{x}(t+T)=\mathbf{x}(t) \quad \Rightarrow \quad E(t+T)=E(\mathbf{x}(t+T))=E(\mathbf{x}(t))=E(t)$.

Therefore, it is possible to replace the non-linear damper by a passive storage element. This was previously shown in [142], but repeated here for completeness.

A C-type storage element, like a spring, has the following constitutive 
relation:

$$
\begin{aligned}
E_{\mathrm{C}} & =\frac{1}{2 \mathrm{C}} q^{2} \\
\dot{q} & =f_{\mathrm{C}} \\
e_{\mathrm{C}} & =\frac{\partial E_{\mathrm{C}}}{\partial q}=q / \mathrm{C},
\end{aligned}
$$

with $E_{\mathrm{C}}$ its energy, $q$ its state and $\mathrm{C}$ its capacity. Its power port is formed by input $f_{\mathrm{C}}$, a flow or generalised velocity; and output $e_{\mathrm{C}}$, an effort or generalised force. The input and output of the buffer can be modulated with a transformer, to a new interconnection port $\left(e_{1}, f_{1}\right)$ :

$$
e_{1}=n \cdot e_{\mathrm{C}} ; \quad f_{\mathrm{C}}=n \cdot f_{1} .
$$

This is a power-continuous interconnection:

$$
P_{\mathrm{C}}=e_{\mathrm{C}} f_{\mathrm{C}}=e_{\mathrm{C}} n f_{1}=e_{1} f_{1}=P_{1} \text {. }
$$

Figure 5.4b shows how the modulated transformer MTF with a buffer element $\mathbf{C}$ can implement the same port behaviour as the nonlinear $\mathbf{R}$, according to the following equations:

$$
e_{1}=n \cdot e_{C} ; \quad n=\frac{-\mu\left(1-x^{2}\right)}{e_{C}} f_{1} \quad \Rightarrow \quad e_{1}=\mu\left(1-x^{2}\right) \dot{x} . \quad \text { (5.23) }
$$

Here $f_{1}=\dot{x}$ is the flow of the 1-junction, thus $\dot{x}$ of the oscillator; and $e_{1}$ is the force applied on the oscillator. By modulating the transformation ratio based on the state $x$ and the buffer's (co-)energy, the port behaviour of this C-MTF- is equal to that of the R-. Now, since the storage element is passive, the complete Van der Pol oscillator is passive.

\section{$\S 3.2$ Influencing the limit cycle period}

The Van der Pol oscillator as modelled in Figure 5.4 is essentially a massspring-damper system, even when the nonlinear damper is "implemented" in a power-continuous way. In order to influence the period of the limit cycle - a requirement for synchronisation as per Theorem 5.2.1 - the mass or spring stiffness could be changed.

The stiffness of a spring can be changed in a proper, power-continuous manner by extending the $\mathbf{C}$-element with a stiffness-changing port. Figure 5.5 shows the augmented spring, with the power-conjugate variables of both ports. The inputs to the spring are the velocity of the Van der Pol oscillator $\dot{x}$ and the rate of stiffness change $\dot{K}$. The related power-conjugate efforts are given by the partial derivative of the spring 
energy with respect to $x$ and $K$, respectively, resulting in $F=K x$ and $e_{\mathrm{K}}=\frac{1}{2} x^{2}$. The Port-Hamiltonian formulation of its dynamics becomes:

$$
\begin{aligned}
H_{C} & =\frac{1}{2} K x^{2} \\
\left(\begin{array}{c}
\dot{x} \\
\dot{K}
\end{array}\right) & =\left[\begin{array}{ll}
1 & 0 \\
0 & 1
\end{array}\right]\left(\begin{array}{l}
f_{\mathrm{m}} \\
f_{\mathrm{K}}
\end{array}\right) \\
y=\left(\begin{array}{c}
e_{\mathrm{m}} \\
e_{\mathrm{K}}
\end{array}\right) & =\left[\begin{array}{ll}
1 & 0 \\
0 & 1
\end{array}\right]\left(\begin{array}{l}
\frac{\partial H_{C}}{\partial x} \\
\frac{\partial H_{C}}{\partial K}
\end{array}\right)=\left(\begin{array}{c}
K x \\
\frac{1}{2} x^{2}
\end{array}\right),
\end{aligned}
$$

where $\left(e_{\mathrm{m}}, f_{\mathrm{m}}\right)$ form the mechanical port with input $f_{\mathrm{m}}=f=\dot{x}$ and output $e_{\mathrm{m}}=F=K x ;\left(e_{\mathrm{K}}, f_{\mathrm{K}}\right)$ form the stiffness-changing port with input $f_{\mathrm{K}}=\dot{K}$ and associated output effort $e_{\mathrm{K}}=x^{2} / 2$.

Note that in any real system it may be difficult to directly change the physical spring stiffness $K$. However, many types of Variable Stiffness Mechanisms or Actuators exist. They employ various means of changing the apparent output stiffness, but in essence they obey the energetic relations given above.

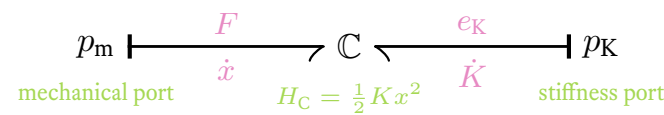

Figure $\mathbf{5 . 5}$ - Spring with variable stiffness, modelled as $\mathbf{C}$-element with two interaction ports: a mechanical port and a stiffness-changing port.

\section{§3.3 Synchronisation of two Van der Pol oscillators}

Two of the Van der Pol oscillators as modelled above will be synchronised. Because the period of each oscillator depends on the spring stiffness $K$, the stiffness-changing port allows changing the period of the limit cycle. According to Theorem 5.2.1, this should allow two Van der Pol oscillators to be synchronised. Note that, because of the effort $e_{\mathrm{K}}$ associated to changing the spring stiffness, changing the period generally does require energy exchange.

However, when synchronising two oscillators, the stiffness change of the two is always opposite: the oscillator that is "behind" in phase must "speed up," i.e. the stiffness must increase; the one that is "ahead" in phase must "slow down," i.e. the stiffness must decrease. Since $x_{i}^{2} \geq 0$, the energy cost associated with the former is $\dot{K}_{1} x_{1}^{2} / 2 \geq 0$, whilst the energy cost associated with the latter is $\dot{K}_{2} x_{2}^{2} / 2 \leq 0$. Therefore, it may be possible to balance out these energies; and it turns out it is. 


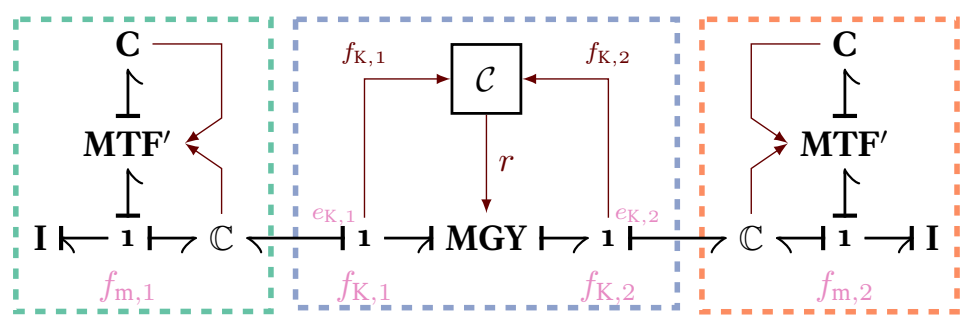

Figure 5.6 - Synchronisation of two Van der Pol oscillators by changing the springs' stiffnesses. $\mathbf{M T F}^{\prime}$ represents the power-continuous modelling as in Figure 5.4b.

By connecting the stiffness-changing ports of two augmented Van der Pol oscillators through a modulating gyrator MGY, as shown in Figure 5.6, the power is routed from one to the other:

$$
\begin{aligned}
\left(\begin{array}{c}
f_{\mathrm{K}, 1} \\
f_{\mathrm{K}, 2}
\end{array}\right) & =\left[\begin{array}{cc}
0 & r \\
-r & 0
\end{array}\right]\left(\begin{array}{l}
e_{\mathrm{K}, 1} \\
e_{\mathrm{K}, 2}
\end{array}\right) \\
P_{1} & =e_{\mathrm{K}, 1} f_{\mathrm{K}, 1}=e_{\mathrm{K}, 1} r e_{\mathrm{K}, 2} \\
P_{2} & =e_{\mathrm{K}_{2}} f_{\mathrm{K}, 2}=-e_{\mathrm{K}, 2} r e_{\mathrm{K}, 1}=-P_{1} .
\end{aligned}
$$

Furthermore, $r$ is a direct input to change both spring stiffnesses in opposite fashion, provided that $x_{i} \neq 0$ :

$$
\begin{aligned}
& \dot{K}_{1}=f_{\mathrm{K}, 1}=r e_{\mathrm{K}, 2}=r x_{2}^{2} / 2 \\
& \dot{K}_{2}=f_{\mathrm{K}, 2}=-r e_{\mathrm{K}, 1}=-r x_{1}^{2} / 2,
\end{aligned}
$$

so if $r>0$ then $\dot{K}_{1} \geq 0$ and $\dot{K}_{2} \leq 0$. Because the MGY is a proper, power-continuous Dirac structure, $r$ is an energy-free, purely modulating control input. lators:

As phase, we can use the polar angle in the phase plane of the oscil-

$$
\phi_{i}=\operatorname{atan} 2\left(\dot{x}_{i}, x_{i}\right) .
$$

("atan2" is the four-quadrant arctangent function.) To synchronise the two oscillators with phase difference $\theta=0$, we use a PD controller to minimise the error $\phi_{2}-\phi_{1} .^{2}$ The transfer function of the tame PD controller, from $\left(\phi_{2}-\phi_{1}\right)$ to $r$, is:

$$
G(s)=K_{\mathrm{P}}\left(\frac{1+s \tau_{\mathrm{D}}}{1+s \beta \tau_{\mathrm{D}}}\right),
$$

${ }^{2}$ Note that the strong nonlinear behaviour of Van der Pol oscillators, combined with the simple phase map $\Phi$, causes $\dot{\phi}_{i}$ to be non-constant, thereby not allowing any other synchronisation than for $\Theta$ identity, i.e. $\theta=\phi_{2}-\phi_{1}=0$. The phase maps could be adapted to have constant $\dot{\phi}_{i}$ and thus allow any phase difference, if that is required. 
where $K_{\mathrm{P}}$ is the proportional gain; $\tau_{\mathrm{D}}$ the derivative gain and $\beta$ the "tameness constant."

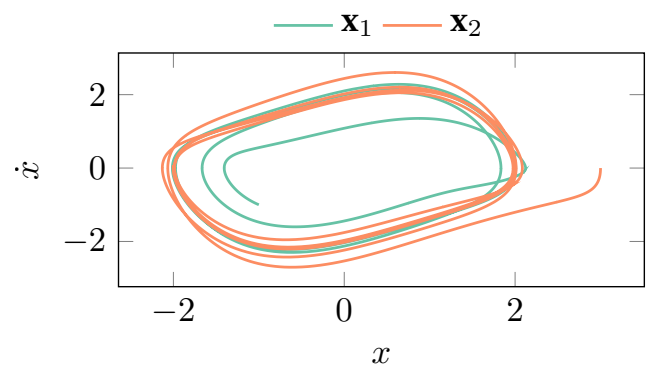

Figure 5.7 - State-space plot of the two Van der Pol oscillators, showing the limit cycle to which both systems converge. (Both spiral in a clockwise direction.)

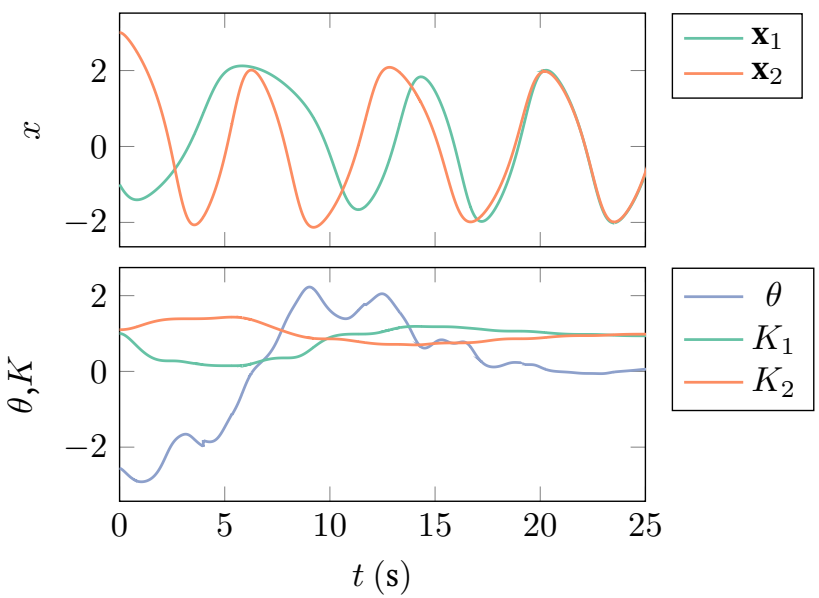

Figure 5.8 - Two synchronised Van der Pol-oscillators. The control algorithm changes the stiffness $K$ of each oscillator, until their phase difference $\theta$ equals 0 . At that point, the two systems oscillate in phase and are synchronised.

The system of Figure 5.6 is simulated in the program 20-sim ([29]) with the parameters as listed in Table 5.1. As Figure 5.7 shows, the two oscillators converge to the same limit cycle, which is expected since they have the same value for $\mu$. Figure 5.8 displays the synchronisation results: the controller achieves synchronisation by modulating the energy flow between the two springs, changing their stiffness in order to make the 


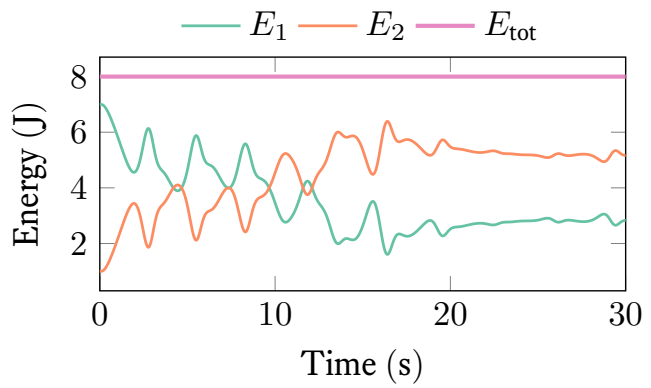

Figure 5.9 - Total energy of the two oscillators, and their sum. The control architecture is power-continuous, so the total energy does not change. Instead, energy is routed from one system to the other to synchronise them.

\begin{tabular}{rcl}
\hline$\mu$ & 0.5 & Van der Pol damping coefficient \\
$K_{\mathrm{P}}$ & 0.012 & Phase controller proportional gain \\
$\tau_{\mathrm{D}}$ & 20.0 & Phase controller derivative gain \\
$\beta$ & 0.1 & Phase controller derivative tameness \\
\hline$K_{1}(0)$ & 1.0 & Initial stiffness of system 1 \\
$K_{2}(0)$ & 1.1 & Initial stiffness of system 2 \\
$x_{1}(0)$ & -1.0 & Initial position of system 1 \\
$\dot{x}_{1}(0)$ & -1.0 & Initial velocity of system 1 \\
$x_{2}(0)$ & 3 & Initial position of system 2 \\
$\dot{x}_{2}(0)$ & 0 & Initial velocity of system 2 \\
\hline
\end{tabular}

Table 5.1 - Parameters used for simulation of the Van der Pol oscillators.

oscillators "catch up" with each other. It is important to state again:

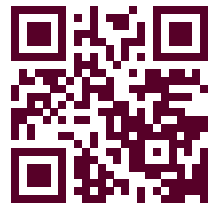

Van der Pol synchronisation the synchronisation is achieved without using any external energy; the control input is a pure modulation of energy flows. The total energy remains constant; see Figure 5.9. This is power-continuous synchronisation.

\section{$\S 4$ Circling masses}

This second example is of two masses that are moving in a 2-D plane. This type of system has no natural limit cycle, so first a limit cycle controller has to be defined for each system $\left(\mathcal{C}_{1}\right.$ and $\mathcal{C}_{2}$ of Figure 5.1). Then a synchronising controller is to be defined for the interconnected sys- 
tems, $\mathcal{C}_{\text {sync }}$ of Figure 5.1. Both are to be achieved in a power-continuous manner.

\section{$\S 4.1$ Limit cycle control}

The intended trajectory for both masses will be the unit circle in the plane. To do this, we will use a method similar to the transverse linearisation approach of [134]. We transform the velocity $\left(v_{x}, v_{y}\right)$ to a component that is parallel to the limit cycle, $v_{\|}$; and a component that is perpendicular to it, $v_{\perp}$. In terms of the extension to tangent maps, of Section 5.2.2: Tangent maps, these are the horizontal and vertical component of $v$, respectively. For the limit cycle control, $v_{\|}$can be left free; $v_{\perp}$ should be controlled to o in such a way that the radius of the circle is equal to 1 . In other words, by considering the position of the mass $(x, y)$ in polar coordinates $(r, \theta)$, the control objective is to steer $r$ to 1 .

Figure 5.10a shows one way to achieve the desired limit cycle: by attaching a spring and damper to $v_{\perp}, v_{\perp}$ will be stabilised in a position depending on the rest length of the spring. However, as with the energyconservative Van der Pol oscillator of Figure 5.4b, it is also possible to save the kinetic energy from the perpendicular direction in a buffer element $\mathbf{C}$, as shown in Figure 5.10b. Subsequently, any energy that is available here can be injected into $v_{\|}$, redirecting all "transverse" or "perpendicular" kinetic energy from the undesired direction $v_{\perp}$, into a direction along the limit cycle, $v_{\|}$. This is similar to the energy routing approach of [38].

A simple simulation with a mass of $1 \mathrm{~kg}$ is carried out to verify the limit cycle control. The projection of the mass's velocity onto the limit cycle is carried out as follows, where $p=(x, y)^{\top}$ is the position of the mass:

$$
\begin{array}{rlr}
p_{\text {intersect }} & =p /\|p\|_{2} & \text { projection onto limit cycle } \\
v_{\mathrm{lc}} & =\left(\begin{array}{cc}
0 & -1 \\
1 & 0
\end{array}\right) p_{\text {intersect }} & \text { unit tangential velocity of limit cycle } \\
v_{\text {perp }} & =\left(\begin{array}{cc}
0 & 1 \\
-1 & 0
\end{array}\right) v_{\mathrm{lc}} & \text { unit perpendicular velocity of l.c. } \\
R & =\left(\begin{array}{ll}
v_{\mathrm{lc}} & v_{\mathrm{perp}}
\end{array}\right)^{\top} & \text { rotation matrix to transform } v .
\end{array}
$$

The first step projects the point $p$ onto the limit cycle; the two rotation matrices rotate this vector to obtain the unit-length tangential and per- 


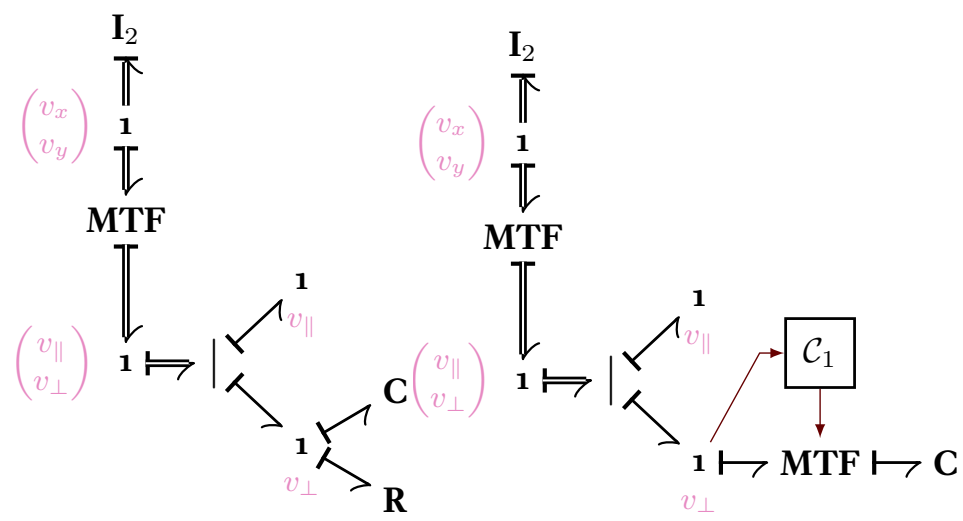

(a) A spring and damper on $v_{\perp}$ cause the nondesired velocity $v_{\perp}$ to go to 0 . (b) By storing the energy from $v_{\perp}$ in a $\mathbf{C}$-element-rather than dissipating it-the energy can be used again.

Figure 5.10 - Limit cycle control for a 2-D rigid body: an MTF maps the $(x, y)$ velocity to a velocity along the limit cycle $\left(v_{\|}\right)$and a velocity perpendicular to the limit cycle $\left(v_{\perp}\right)$.

pendicular velocities. Because these are unit length, they can be used to construct a rotation matrix $R$ that transforms $\left(v_{x}, v_{y}\right)$ into $\left(v_{\|}, v_{\perp}\right)$. This rotation matrix is the transformation matrix of the MTF of Figure 5.10.

The simulation results in Figure 5.11 show how the mass starts with an initial velocity $v_{0}$ that is not on the unit circle. It can be seen from transformed velocity $\left(v_{\|}, v_{\perp}\right)$ of Figure $5.11 \mathrm{~b}$ that the kinetic energy of the perpendicular direction is extracted and directed into the tangential direction. The plot of $(x, y)$ of Figure 5.11a shows the convergence to the limit cycle.

\section{$\S 4.2$ Synchronisation controller}

Because the limit cycle of the moving mass is the unit circle, the phase map $\Phi$ is not hard to define: $\phi$ is the angle of the polar coordinates of $(x, y)$ and $\theta$ is the difference of the angles.

$$
\phi_{i}=\operatorname{atan} 2\left(y_{i}, x_{i}\right) ; \quad \theta=\phi_{2}-\phi_{1} .
$$

$\Phi$ is actually a diffeomorphic map: the velocity $\left(v_{x}, v_{y}\right)$ is transformed with $R$ of $(5.37)$ to $\left(v_{\|}, v_{\perp}\right)$, of which $v_{\|}$is the velocity projected along the limit cycle: the horizontal component of $v$. This means that the synchronisation controller can act directly on $v_{\|}$: if $v_{\|, 1}=v_{\|, 2}$, then 


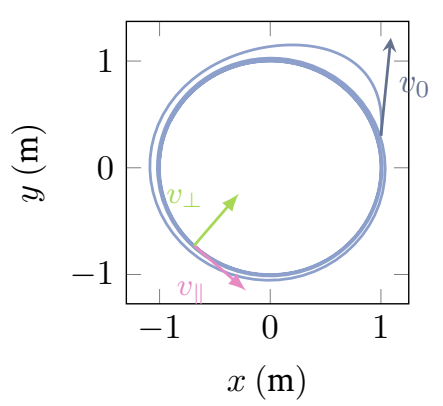

(a) Motion of the mass in the 2-D plane. The initial velocity $v_{0}$ is indicated, as are $v_{\|}$and $v_{\perp}$ in one location.

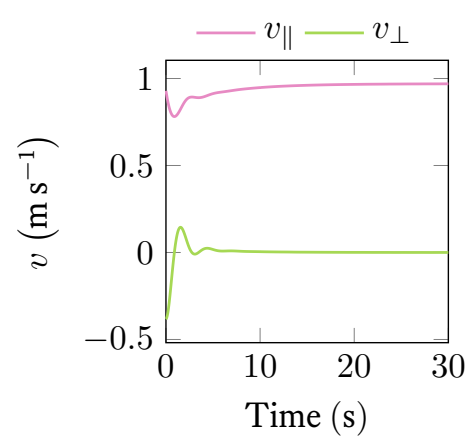

(b) The controller extracts energy from the perpendicular direction and redirects it to the tangential direction.

Figure 5.11 - Limit-cycle control of a mass moving in a 2-D plane, counterclockwise along the unit circle. The controller is power-continuous, neither injecting nor extracting energy.

$\dot{\theta}=0$ and the systems are synchronised. The control effort is dual to $\dot{\theta}$ and can thus be transformed back to the space of $\left(v_{x}, v_{y}\right)$ using the transpose of map $R$.

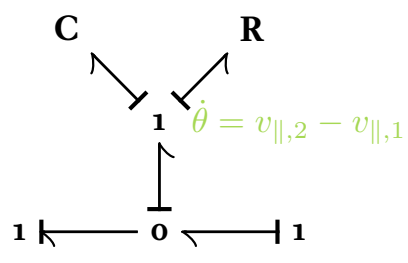

Figure 5.12 - Synchronisation controller strategy: a spring and damper between $v_{\|}$of the two systems will cause their tangential velocities to become equal and hence the systems to become synchronised.

A possible synchronisation controller is shown in Figure 5.12: the spring and damper will cause $\dot{\theta}$ to go to zero, while the phase difference $\theta$ will depend on the rest length of the spring $\mathbf{C}$. Although this is a passive synchronisation controller, we do not want to dissipate energy in the damper. Hence, the same approach as before is taken: the control actions are determined by a modulation of the power flow between the system and a buffer, as with the power-continuous Van der Pol oscillator. Any 
energy that is extracted due to e.g. a damping action can be re-used and injected somewhere else. Figure 5.13 shows the model structure of two synchronised circling masses: the limit cycle and synchronisation controllers have been taken together into a single MTF-element for each system; all power flows are directed into a central buffer.

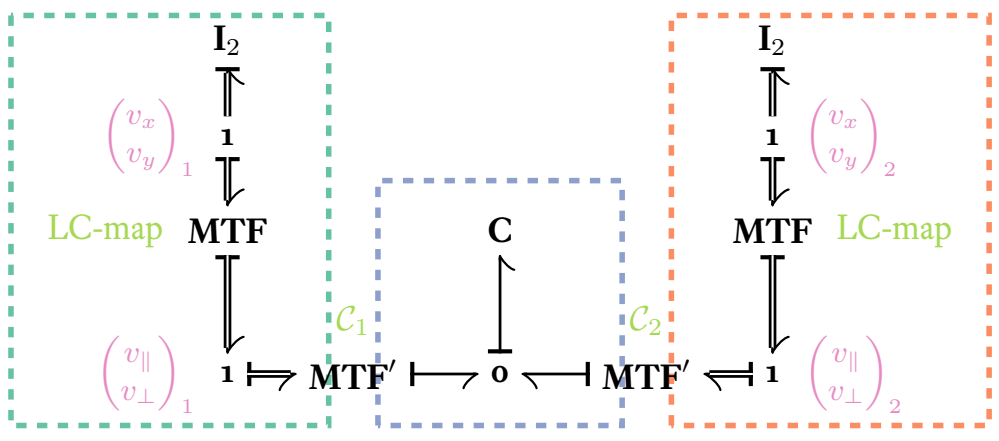

Figure 5.13 - Limit cycle and synchronisation control: the central C-element acts as a buffer for the power-continuous control laws that are implemented as modulated transformers, the $\mathbf{M T F}^{\prime}$ elements. Because the phase map is diffeomorphic, the velocity can be mapped ("LC-map") to transverse and tangential components. This allows for straightforward limit cycle and synchronisation control on these velocities.

The two $\mathbf{M T F}^{\prime}$ elements direct the energy flow as follows:

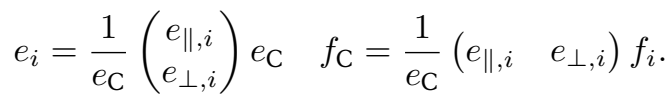

$\left(e_{\mathrm{C}}, f_{\mathrm{C}}\right)$ are the port variables of the buffer $\mathbf{C}$-element; $\left(e_{i}, f_{i}\right)$ the $(2-$ dimensional) port variables of system $i-\operatorname{so} f_{i}=\left(v_{\|}, v_{\perp}\right)^{\top} . e_{\|}$and $e_{\perp}$ are required control forces for the limit cycle generation $\left(e_{\perp}\right.$, dual to the transverse velocity) and synchronisation $\left(e_{\|}\right.$, dual to the tangential velocity). These forces are generated by $\mathrm{P}(\mathrm{I}) \mathrm{D}$ control laws as follows:

$$
\begin{aligned}
& e_{\perp, i}=K_{\mathrm{P}, \perp}\left(1+\frac{1}{s \tau_{\mathrm{I}, \perp}}\right)\left(\frac{1+s \tau_{\mathrm{D}, \perp}}{1+s \beta \tau_{\mathrm{D}, \perp}}\right)\left(1-r_{i}\right) \\
& e_{\|, 1}=-K_{\mathrm{P}, \|}\left(\frac{1+s \tau_{\mathrm{D}, \|}}{1+s \beta \tau_{\mathrm{D}, \|}}\right)\left(\theta_{s}-\theta\right) \\
& e_{\|, 2}=+K_{\mathrm{P}, \|}\left(\frac{1+s \tau_{\mathrm{D}, \|}}{1+s \beta \tau_{\mathrm{D}, \|}}\right)\left(\theta_{s}-\theta\right) ;
\end{aligned}
$$

$\theta_{s}$ is the desired phase difference and $\left(1-r_{i}\right)$ is the radial distance of mass $i$ to the unit circle/limit cycle. Note that the limit cycle controllers 
are identical for the two systems, while the synchronisation controllers differ by a sign, resulting from the definition of $\theta=+\phi_{2}-\phi_{1}$. These are regular $\mathrm{P}(\mathrm{I}) \mathrm{D}$ control laws, but the output is used for a pure modulation of the $\mathbf{M T F}^{\prime}$ elements. Hence, all control actions require no external energy whatsoever.

Of course, (5.39) is only well-defined when $e_{\mathrm{C}} \neq 0$, i.e. when there is energy in the buffer element. Any energy used by the control action can come from this buffer, but as is demonstrated by the next experiment, the buffer indeed only ever acts as a small, temporary buffer; not as a large energy source.

\section{$\S 4.3$ Synchronisation experiment}

\begin{tabular}{|c|c|c|c|}
\hline Parameter & Value & & Description \\
\hline$m_{i}$ & 1 & $\mathrm{~kg}$ & Mass \\
\hline$\overline{K_{\mathrm{P}, \perp}}$ & 5 & & Proportional gain of limit cycle controller \\
\hline$\tau_{\mathrm{D}, \perp}$ & 1 & $\mathrm{~S}$ & Derivative time constant limit cycle controller \\
\hline$\tau_{\mathrm{D}, \perp}$ & 1 & $\mathrm{~s}$ & Integral time constant of limit cycle controller \\
\hline$K_{\mathrm{P}, \|}$ & 1 & & Proportional gain of synchronisation controller \\
\hline$\tau_{\mathrm{D}, \|}$ & 1 & s & Derivative time constant of sync. controller \\
\hline$\beta$ & 0.1 & & Derivative tameness of all $\mathrm{P}(\mathrm{I}) \mathrm{D}$ controllers \\
\hline$E_{\mathrm{C}}(0)$ & 1.5 & $\mathrm{~J}$ & Initial energy present in buffer \\
\hline$p_{1}(0)$ & $\left(\begin{array}{c}1 \\
0.3\end{array}\right)$ & $\mathrm{m}$ & Initial position of mass 1 \\
\hline$p_{2}(0)$ & $\left(\begin{array}{l}0 \\
1\end{array}\right)$ & $\mathrm{m}$ & Initial position of mass 2 \\
\hline$v_{1}(0)$ & $\left(\begin{array}{c}0.1 \\
1\end{array}\right)$ & $\mathrm{m}$ & Initial velocity of mass 1 \\
\hline$v_{2}(0)$ & $\left(\begin{array}{l}-0.5 \\
-0.3\end{array}\right)$ & $\mathrm{m}$ & Initial velocity of mass 2 \\
\hline
\end{tabular}

Table 5.2 - System and control parameters of the simulation experiment of Figure 5.13. The control parameters are not optimised for fast response, to better show the effect of control actions.

The system of Figure 5.13 is simulated using the phase mapping of (5.37) and (5.38); the power-continuous control laws of (5.39) and (5.40); and the parameters as listed in Table 5.2. Figure 5.14 displays the results of this simulation: the position of each mass, the phase difference and the projected velocities.

Initially, neither of the masses is moving along the limit cycle, but the 


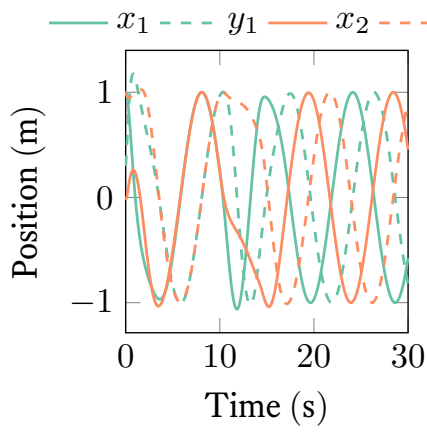

(a) Movement of the two masses.

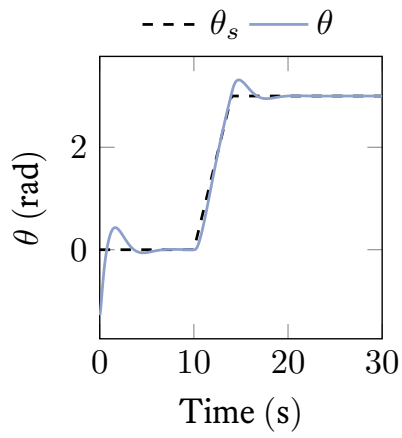

(c) Phase difference.

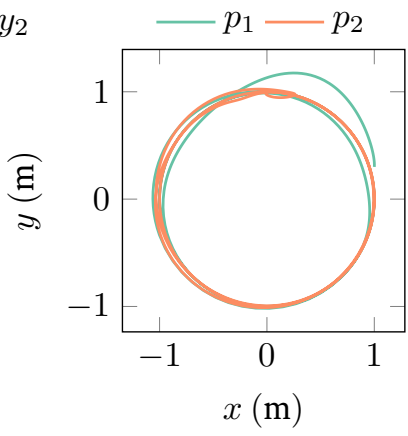

(b) Trajectories in $(x, y)$ plane.

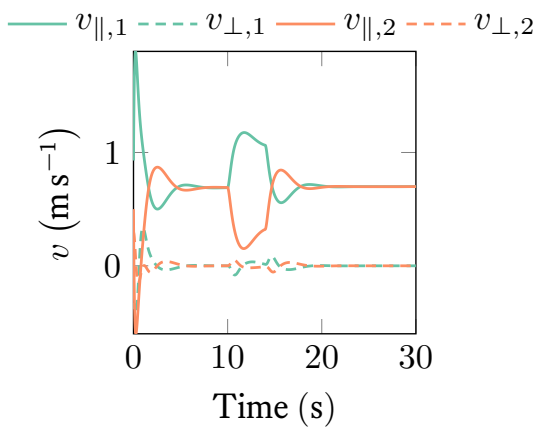

(d) Projected velocities.

Figure 5.14 - Synchronisation of 2-D counter-clockwise circling masses. The limit cycle control steers both masses into a trajectory along the unit circle. The synchronisation controller tracks the phase difference setpoint $\theta_{s}$. 
control algorithms quickly steer them in a trajectory along the unit circle, while the phase difference is regulated to $\theta=0$. After $10 \mathrm{~s}$, the desired phase difference $\theta_{s}$ is gradually increased to $\pi$, which the systems are able to follow. The projected velocities of Figure 5.14d give insight into how the synchronisation is achieved: one of the masses is slowed down, while the other is sped up.

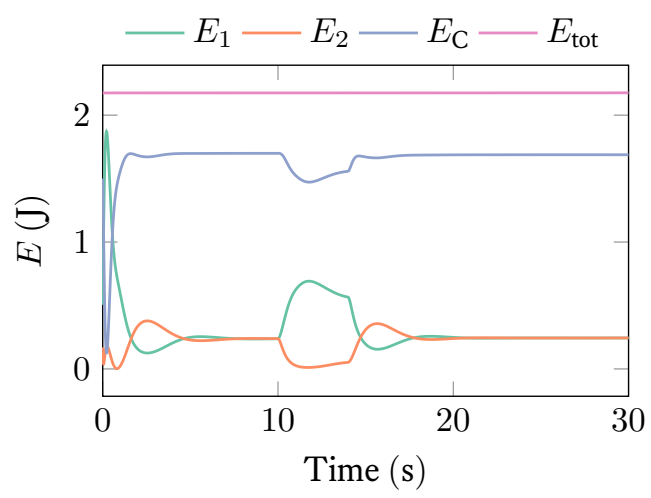

Figure 5.15 - Energy present in the system during the limit cycle synchronisation experiment. The total energy is constant, showing that this is indeed a power-continuous synchronisation scheme-no external energy source is required. The energy in the internal buffer $E_{\mathrm{C}}$ is first required to direct the masses along the unit circle and synchronise them; later to change the phase difference. However, this level quickly returns to its original value.

Very important is Figure 5.15, which shows the energy present in the system: kinetic energy of mass $1\left(E_{1}\right)$ and mass $2\left(E_{2}\right)$; and the energy in the buffer element $\left(E_{\mathrm{C}}\right)$. The total energy $E_{\text {tot }}$ is constant, because the control actions are a pure modulation and neither inject nor extract energy. The plot also makes clear that the buffer element is indeed only ever used as a temporary buffer, not as a built-in energy source.

\section{$\S 5$ Conclusion}

Synchronised circling masses

In this paper we have taken another look at synchronisation of periodic systems. Our geometric definition of synchronisation is a coordinateindependent formulation of the synchronisation phenomenon; it can be used to define synchronisation of any two arbitrary limit cycles. The concept of "phase" is inherent in the definition and, when expressed in coordinates, the "phase difference" $\theta$ arises naturally. An extension to 
synchronisation of $n$ systems can be made easily by defining multiple maps $\Theta$. From the background of morphological computation, we have focussed on control by interconnection and power-continuous synchronisation.

Our definition of synchronisation is very generic; the choice of the phase maps $\Phi_{i}$ depends on the specific systems that are synchronised. Therefore, we have presented two examples in which we first created a limit cycle and then synchronised two of these systems. Both were achieved in a power-continuous fashion, i.e. without using external energy - both the limit cycle and synchronisation controllers are passive systems using control-by-interconnection. In future work, we want to investigate control and controllability aspects of the horizontal and vertical planes: in the "circling masses" example, the control distribution $g(x)$ always covers the full tangent space. It will be interesting to see what control methods are necessary when this is not the case.

We are currently applying the synchronisation strategies presented in this work on a quadrupedal running robot. In previous work ([46]), the phase between legs was uncontrolled and, especially in real-world experiments, unstable. With more control over the phase, and thus the gait, we already see better performance of the robot. In the near future, we hope to present a successful, energy-efficient quadruped that runs with synchronised legs, with morphological computation through control-by-interconnection. 


\section{Chapter 6}

\section{Synthesis}

Biomimetic or bio-inspired robots are designed by taking inspiration from nature. Morphological computation means that the robot's natural dynamics show desired behaviour, sharing control between "brain" and "body." When doing biomimetic morphological computation, the challenge is to identify nature's dynamic principles and transfer these to a robot.

This chapter concerns biomimetic morphological computation for a cheetah robot, focussing on the role of the spine. The spine's dynamic principles are identified from analysis of a running cheetah video and synthesised in a robot model. The robot is fully symmetric, except for the spine, such that any locomotion behaviour can be attributed to the spine.

In both simulation and real-world experiment, the model runs forwards or backwards determined by the spine configuration. The robot's natural dynamics result in locomotion behaviour: the model successfully runs due to the dynamic, biomimetic morphological computation.

This chapter has been submitted to the Bioinspiration \& Biomimetics journal in March 2017. 


\section{$\S 1$ Introduction: on quadrupeds and the spine}

When asked what a quadrupedal robot is, one usually responds with "a four-legged robot." Indeed, the name draws attention to the four pedes or feet, which may have caused many a researcher to focus on the legs of their quadruped. Are they not missing out by ignoring the spine?

A whole range of impressive cheetah robots consists of a single rigid body with four legs attached: from the MITCheetah to the HyQ robot and the entire line-up of Boston Dynamics [41, 97, 129]. And this while Gracovetsky went so far as to state that legs only move for ground clearance and terrain adaptability, while the spine does all the hard running work [54]! It must of course be acknowledged that these mentioned robots are truly impressive systems that indeed achieve quadrupedal running. However: could they do even better with a spine?

Taking their inspiration from nature, many other scientists have investigated robotic quadrupeds with a spine: studying linear or rotational spinal joints, actuated and unactuated versions, or passive slope-walkers $[77,111,33,76]$. It is clear that a spine can contribute positively to locomotion. Our question is: can it cause locomotion?

Some researchers are going so far as Gracovetsky's extreme, by placing the only actuator in the spine [170]. By varying the pattern generating weights in the spine, this robot is able to show multiple, robust gait patterns [171]. Some questions raised in this work are: what are the benefits of an asymmetrical spine? And: what can actuated legs contribute to spine-induced locomotion?

Our research question is: can a passive spine cause locomotion in a quadrupedal robot where everything-mass distribution, kinematics, control-is symmetric; with the only asymmetry introduced by the spine?

\section{§1.1 Morphological computation and the spine}

Morphological computation means that the robot's natural dynamics show desired behaviour. In this case, the aim is to design a quadruped with a spine, where the spine dynamics cause locomotion. This has two benefits: firstly, exploiting the natural dynamics should lead to energy efficiency; secondly, control should be easier, since it will only have to excite or stabilise the natural dynamics.

In previous work and inspired by the motion of a running cheetah, we ran computer simulations of a simple two-legged sagittal-plane quadruped that consisted of two mirrored halves, coupled by an elastic spine. The Centre of Compliance and stiffness of the spine were varied to see if it would induce locomotion and indeed, there were certain configurations that led to running [46]. However, the 6-DoF spine spring of the computer model is difficult to implement practically, and the morpho- 
logy was inspired by casual observation of a running cheetah. The new research, presented in this chapter, seeks to do biomimetic morphological computation: extract the required morphology from a thorough, quantitative analysis of a running cheetah.

The challenge in bio-inspired robotics is always choosing what to copy from nature, and how to implement that in an artificial system [56]. Or, as Pfeifer put it in 2007 [104]:

"Simply copying a biological system is ... of little interest ... . Rather, the goal is to work out principles of biological systems and transfer those to robot design. "

The final goal of the presented research is to mimic the high speed and energy efficiency of a running cheetah. When working out the principles of the cheetah, therefore, we shall focus on its movement and dynamics, rather than simply its kinematics. Hopefully, we can transfer these observations to a quadrupedal robot with a spine, where most - if not all-of the locomotion is achieved through morphological computation. The steps of this methodology are:

1. quantitatively analysing a video of the running cheetah;

2. calculating the relevant dynamics of the motion;

3. abstracting governing principles from those dynamics;

4. synthesising the principles into a robot design;

5. performing experiments with the robot, both in simulation and real experiments, to obtain running behaviour with minimal control effort.

The most important contributions of this chapter are:

- quantitative biomimetic kinematic and dynamic analysis of a running cheetah;

- successful morphological computation by a compliant spine: locomotion resulting solely from the passive, asymmetric spine.

\section{$\S 1.2$ Tools}

When studying the motion of the cheetah, we shall use tools from differential geometry: homogeneous transformations, twists and wrenches. The notation is as follows:

- $\Psi_{A}$ is a 2-D Euclidian coordinate frame. 
- $H_{A}^{B} \in S E(2)$ is the homogeneous transformation from $\Psi_{A}$ to $\Psi_{B}$.

- ${ }^{C} T_{A}^{B} \in \mathfrak{s e}(2)$ is the twist (generalised velocity) $\left(\begin{array}{lll}\omega & v_{x} & v_{y}\end{array}\right)^{\top}$ of $\Psi_{A}$ w.r.t. $\Psi_{B}$, expressed in $\Psi_{C}$.

- ${ }^{B} W_{A} \in \mathfrak{s e}^{*}(2)$ is a wrench (generalised force) $\left(\begin{array}{lll}\tau & F_{x} & F_{y}\end{array}\right)$ acting on $A$, expressed in $\Psi_{B}$.

- $\mathcal{P}$ is the generalised momentum co-vector.

One benefit of using differential geometry is that the dynamics of rigid bodies, dampers and springs can easily be expressed as Port-Hamiltonian Systems (PHS), with as ports the conjugate power pair $W$ and $T$. The interconnection of these PHS is characterised by a power-continuous Dirac structure [123].

Although the PHS formulation - and its graphical expression in bond graphs - is beyond the scope of this chapter, the applicability of these frameworks is striking: morphological computation is about the proper interconnection of various energy-storing elements (masses, springs); PHS and bond graphs explicitly model and show these energy-storing elements and their interconnection!

\section{$\S 2$ Identification}

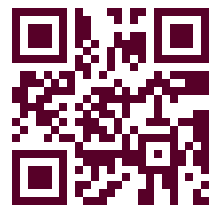

The running cheetah

* The animal conveniently provided distinct spots to track.
To study the natural kinematics and dynamics, we analyse a video of a running cheetah. To focus on the role of the spine in locomotion, the torso of the cheetah is considered as two rigid bodies - an anterior body $A$ and posterior body $P-$ connected by the spine.

\section{$\S 2.1$ Video analysis}

To extract the information from the video (see Figure 6.1 for a single frame), we execute the following steps:

1. With Matlab's video processing toolbox, track points* on the hip, spine and shoulder of the cheetah.

2. Use these points to obtain $\Psi_{A}$ and $\Psi_{P}$, frames attached to the anterior and posterior body of the cheetah, expressed as $H_{A}^{0}(t)$ and $H_{P}^{0}(t)$.

3. Obtain the twist of the bodies using a numerical approximation of $\dot{H}$ as follows:

$$
{ }^{A} \tilde{T}_{A}^{0}(t)=H_{0}^{A}(t) \cdot \hat{\dot{H}}_{A}^{0}(t) .
$$




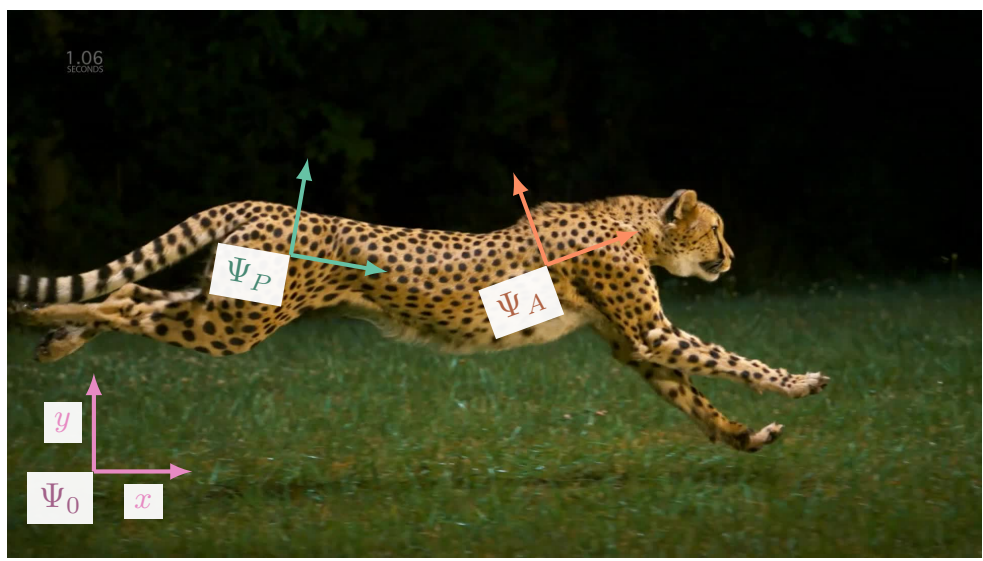

Figure 6.1 - A single frame from the video, showing the cheetah and the frames placed on its "anterior body" $\Psi_{A}$ and "posterior body" $\Psi_{P}$; and the reference frame $\Psi_{0}$. Video from [165].

4. Find the wrenches ${ }^{A} W_{A}(t)$ and ${ }^{P} W_{P}(t)$ using inverse dynamics according to (6.3).

5. Split the time series into "strides," extracting the periodic trajectories of $H_{A}^{0},{ }^{A} T_{A}^{0},{ }^{A} W_{A}$ and those corresponding to the posterior body.

Newton's second law in $S E(2)$ is simply ${ }^{0} \dot{\mathcal{P}}={ }^{0} W$, or in principal inertia frame $\Psi_{A}$ (where the inertia tensor $\mathcal{I}$ is constant and diagonal):

$$
{ }^{A} \dot{\mathcal{P}}^{\top}={ }^{A} \mathcal{I} \cdot{ }^{A} \dot{T}_{A}^{0}=a d_{A}^{\top}{ }_{T_{A}^{0}}{ }^{A} \mathcal{P}^{\top}+{ }^{A} W^{\top},
$$

where $a d_{A} T_{A}^{0}$ is the adjoint representation of the Lie algebra $\mathfrak{s e}(2)$.

An estimate of the cheetah's mass properties gives ${ }^{A} \mathcal{I}$, which is used to calculate the wrench exerted on the body, ${ }^{A} W$ :

$$
{ }^{A} W^{\top}={ }^{A} \mathcal{I} \cdot{ }^{A} \dot{T}_{A}^{0}-a d_{A}^{\top} T_{A}^{0}{ }^{A} \mathcal{P}^{\top} .
$$

\section{$\S 2.2$ Observed principles: Twist}

The result of step 5 is shown in Figure 6.2: the twist of the anterior and posterior body as observed in the 5 full strides that the video shows, normalised in time with the observed period $T$. While there is some variation in the magnitude of the forward velocity $v_{x}$, the trajectories are consistent enough to extract a "stride trajectory" for the cheetah. 


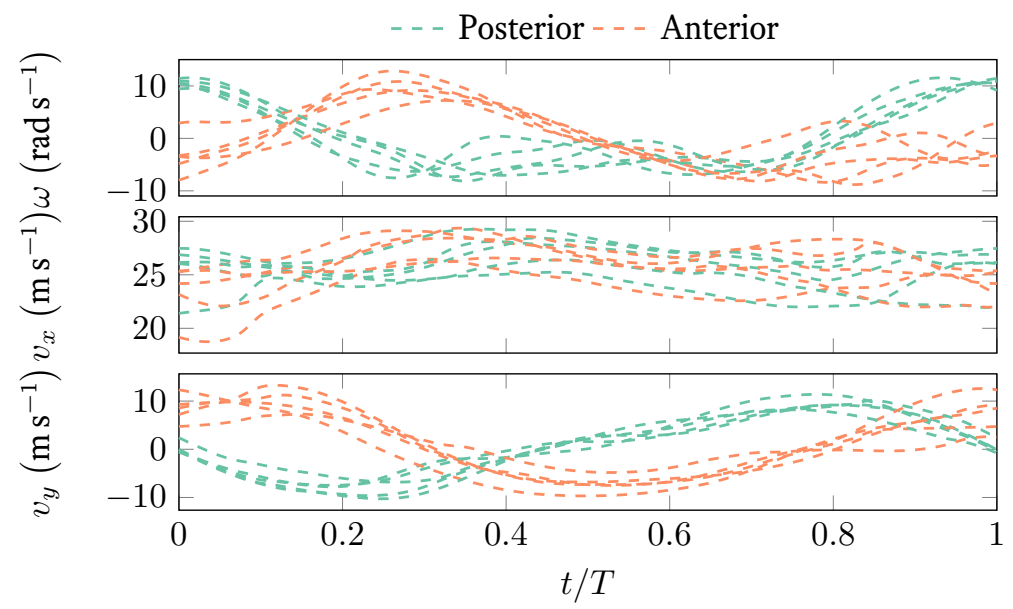

Figure 6.2 - Twist of anterior and posterior body in the video: split in 5 strides, overlaid in normalised time $t / T$. Although there is some spread in the forwards velocity $v_{x}$, the shape is similar in each stride; and $\omega$ and $v_{y}$ overlap very well. The phase difference between anterior and posterior body is very clear.

Focussing on the spine, the relative velocity of the anterior body and posterior body is most interesting. This twist is easily calculated (6.4) and is plotted in Figure 6.3a. It is the result of the complicated arching and bending of the spine, but can it be described in a simpler way?

$$
{ }^{P} T_{A}^{P}={ }^{P} T_{A}^{0}-{ }^{P} T_{P}^{0}=A d_{H_{A}^{P}}{ }^{A} T_{A}^{0}-{ }^{P} T_{P}^{0}
$$

* Sometimes attributed to Chasles, it states that every Euclidean motion is a screw displacement along a screw axis.

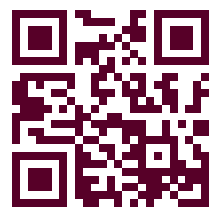

Motion of anterior and posterior body, with ICR shown
All motions in 2-D can be described as a pure rotation around some point, the Instantaneous Centre of Rotation (the projection of Mozzi's theorem* on the plane). Figure 6.3b shows the calculated ICR of ${ }^{P} T_{A}^{P}$, expressed in $\Psi_{P}$.

Except for when $\omega \approx 0$ - where the ICR necessarily moves towards infinity - and around $t / T \approx 0.6$, where the relative velocity is nearly zero, the ICR appears in a nearly constant location. This means that the complicated spine motion is actually largely a pure rotation around a fixed point, somewhere above the back of the cheetah! Figure 6.3a shows that the linear velocity is indeed nearly zero in this point. 


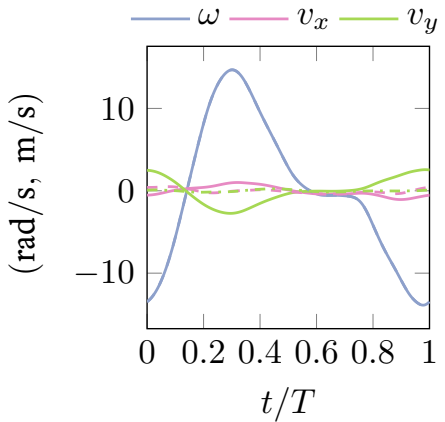

(a) The twist ${ }^{P} T_{A}^{P}$ itself (solid) and when expressed in a spine frame $\Psi_{S}\left({ }^{S} T_{A}^{P}\right.$, dashed).

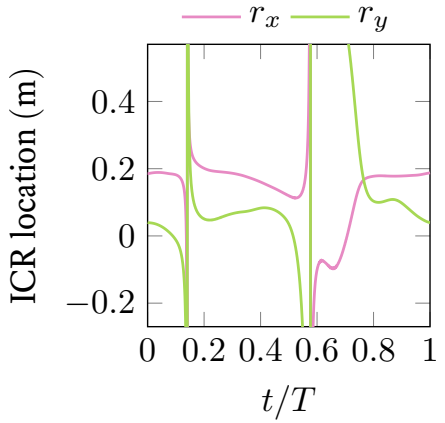

(b) The location of the Instantaneous Centre of Rotation (ICR), expressed as $r$ in $\Psi_{P}$.

Figure 6.3 - Relative velocity between anterior and posterior bodies $\left({ }^{P} T_{A}^{P}\right)$. It turns out that the complicated spine motion is mostly a pure rotation around a virtual point fixed somewhere above the cheetah's back. $\Psi_{S}$ is placed in this point; (a) shows that the linear velocity there (dashed line) is indeed practically zero.

\section{$\S 2.3$ Observed principles: Wrench}

Although we do not have any measurements of ground reaction forces in the cheetah, an estimate estimate of the wrench that the spine exerts on the anterior and posterior body can be obtained by subtracting the wrenches on the two bodies, found by inverse dynamics calculation. These wrenches are the sum of the spine wrench ${ }^{S} W_{\bullet, S}$ and a leg wrench ${ }^{S} W_{\bullet}, L$, where the spine wrench has a different sign in the anterior and posterior bodies:

$$
\begin{aligned}
{ }^{S} W_{A} & ={ }^{S} W_{A, L}+{ }^{S} W_{A, S} \\
{ }^{S} W_{P} & ={ }^{S} W_{P, L}-{ }^{S} W_{P, S} \\
{ }^{S} W_{A}-{ }^{S} W_{P} & =2{ }^{S} W_{S}+{ }^{S} W_{A, L}-{ }^{S} W_{P, L}
\end{aligned}
$$

Figure 6.4 shows this wrench difference of (6.7) together with the angle between the anterior and posterior body, $\theta$. The correlation is striking: a rotational spring in this location can supply most of the required torque, as shown in Figure 6.4b. Adding this spring significantly lowers the force that the legs $\left({ }^{S} W_{\bullet, L}\right)$ will have to generate!

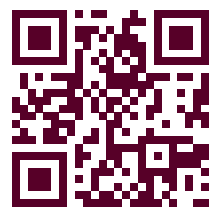

First attempt with a spine joint i.s.o. RCC spring 


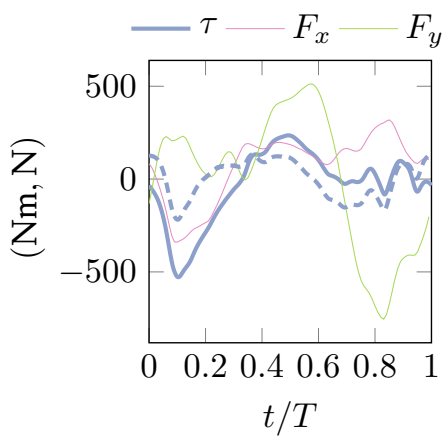

(a) ${ }^{S} W_{P}-{ }^{S} W_{A}$ : the torque $\tau$ can be significantly reduced by adding a rotational spring in the spine (dashed line).

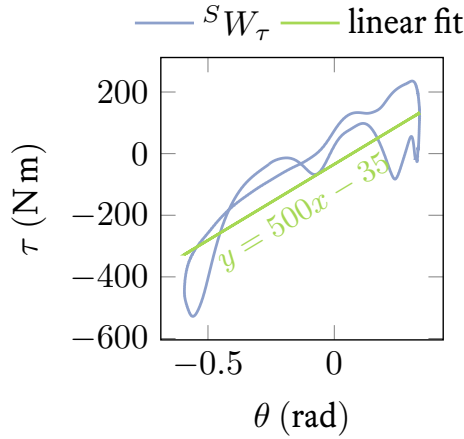

(b) A least-squares linear fit on the torque from (a) gives a spring with $\tau=500(\theta-$ $0.07)$.

Figure 6.4 - The wrench that the spine could exert, from (6.7), correlates strongly with the rotation between anterior and posterior body $\theta$ : most of it could be supplied by a passive rotational spring.

\section{§3 Synthesis}

We have learned two lessons from the cheetah analysis:

1. the spine motion is largely a pure rotation around a fixed point; and

2. a major part of the force exerted by the spine can be supplied by a rotational spring.

The challenge now is to transfer these lessons to robot design: the synthesis. The abstract dynamic model shown in Figure 6.5 incorporates the minimal requirements to apply the two lessons:

\section{Passive compliant spine joint with adjustable position}

This is the morphological tuning parameter that is the only asymmetry in the model. The location is adjustable to find the proper ICR for the model, and study its effect on locomotion.

\section{SLIP-like legs}

Many studies have shown that running legs can be modelled and built as Spring-Loaded Inverted Pendulum (SLIP). The feet should describe a linear motion. 


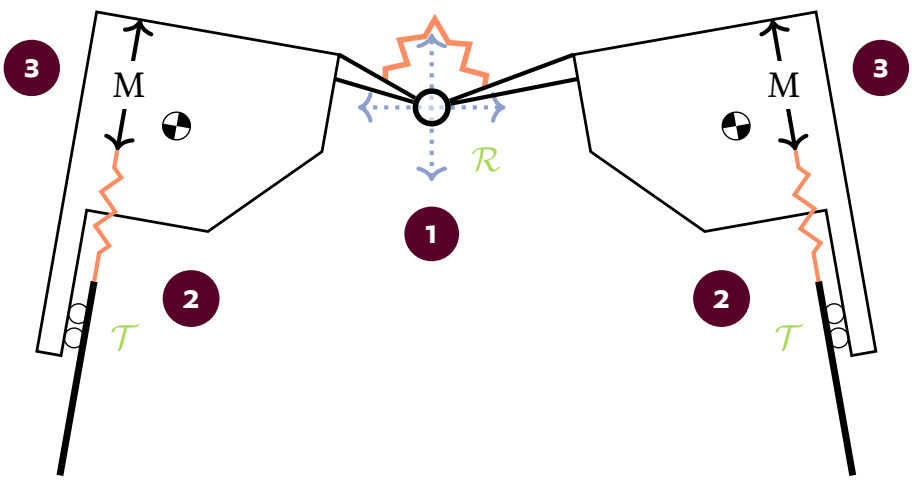

Figure 6.5 - Abstract model synthesised from the observed dynamic principles: two SLIP-like legs (translational joints $\mathcal{T}$ ) with series-elastic connection to the motor $M$; connected through a passive compliant spine (rotational joint, $\mathcal{R}$ ) whose location can be adjusted. The numbers indicate the three requirements explained in the text.

\section{Energy injection}

Each leg will have an electric motor for energy injection, to excite and sustain the natural dynamics. In order not to disrupt those dynamics, it should be elastically coupled to the feet (series elastic coupling), or be fully backdrivable (parallel actuation).

\section{$\S 4$ Robot}

The abstract dynamic model of Figure 6.5 is translated into a constructible robot design. In addition to the three principles from the preceding section, the design should have:

1. a way to constrain out-of-plane motion;

2. an easy-to-produce design; and

3. a light-weight construction in order to limit actuation requirements.

All these requirements are translated in the design shown in Figure 6.6 (single hopper, or half the robot) and Figure 6.7 (full robot). The most important design features are highlighted below; Table 6.1 lists important parameters.

Evans' mechanism The linear leg motion is obtained by use of Evans' mechanism, a four-bar linkage that approximates a straight line in a

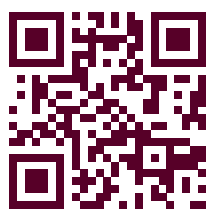

Assembly of the single hopper 


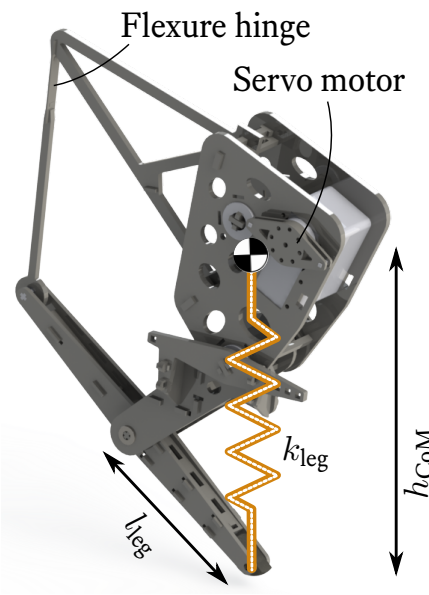

(a) Render of the single hopper. The prismatic spring is the stiffness felt at the foot, caused by the series elastic elements drawn in (b).

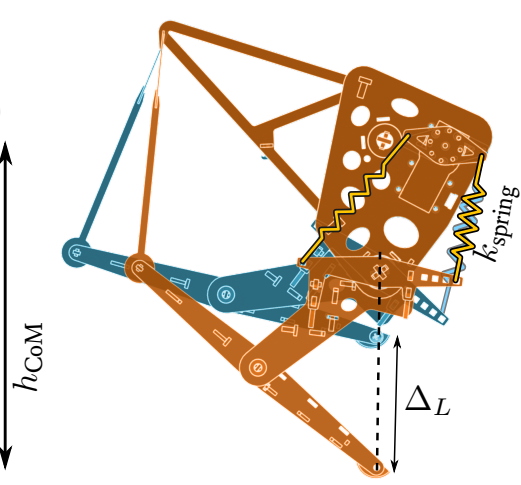

(b) The two extreme positions of Evans' mechanism. The servo motor is connected to the leg mechanism through series elastic elements.

Figure 6.6 - A single hopper, or half the cheetah robot. It is connected to the spine assembly just north-west of the CoM. 


\begin{tabular}{llr}
\hline Property & Value & Description \\
\hline$m_{\text {body }}$ & $0.40 \mathrm{~kg}$ & Mass of single body, including motor \\
$m_{\text {leg }}$ & $0.07 \mathrm{~kg}$ & Mass of single leg (Evans' moving parts) \\
$l_{\text {leg }}$ & $0.10 \mathrm{~m}$ & Length of each leg segment \\
$h_{\mathrm{CoM}}$ & $0.22 \mathrm{~m}$ & Height of hopper's CoM at rest \\
$k_{\text {spring }}$ & $2570 \mathrm{~N} \mathrm{~m}^{-1}$ & Series elastics stiffness \\
$k_{\text {leg }}$ & $200 \mathrm{~N} \mathrm{~m}^{-1}$ & Equivalent stiffness at foot \\
\hline$d$ & $0.30 \mathrm{~m}$ & Distance between feet at rest \\
$h_{\text {spine }}$ & $0.30 \mathrm{~m}$ & Height of spine in topmost configuration \\
\hline
\end{tabular}

Table 6.1 - Robot parameters. The first five concern a single hopper (Figure 6.6); the last two refer to the full cheetah of Figure 6.7.

limited range of motion. It is the simplest mechanism that can describe (almost) linear motion using revolute joints, with low friction and good constructibility.

Series Elastic Actuation (SEA) While parallel actuation as used in [46] is theoretically better for exploiting natural dynamics, previous work showed that in practice there is a detrimental trade-off between motor operability and backdrivability. [163]

Rapid prototyping Laser-cut Delrin ${ }^{\mathrm{TM}}$ (PolyOxyMethylene or POM) and $3-D$ printed $A B S$ are used as main construction materials. They are light-weight materials, and using these rapid prototyping methods it is possible to build the robot in-house with a lead time of two days.

Adjustable spine joint location The location of the spine joint can be adjusted in the vertical direction using predetermined holes for the attachment of the spine elements. Horizontal variation is achieved by the use of spine elements with various lengths.

Boom fixture Out-of-plane motion is constrained by a boom attached to the spine joint. The robot can rotate freely around the boom axis; the base of the boom is mounted on a central pole, releasing the other two rotational degrees of freedom. This way, the robot can move on a semi-sphere shell that approximates the sagittal plane with a long enough $(1 \mathrm{~m})$ boom.

Springs Antagonistically mounted linear (prismatic) springs are used to create a torsional compliance at the spine joint. 


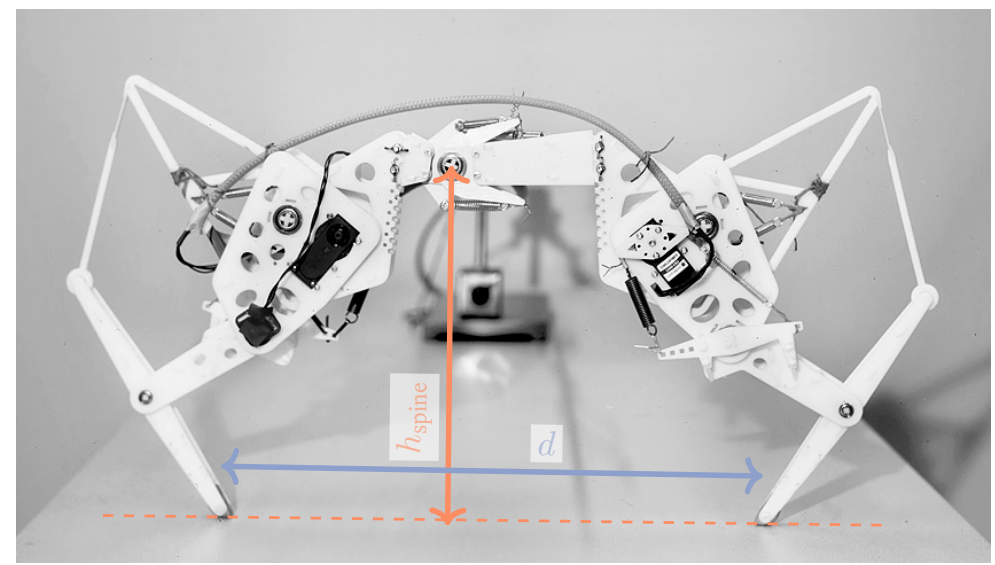

Figure 6.7 - Full cheetah model, consisting of two mirrored hoppers and the spine in-between. In the background: the central pole around which the cheetah runs. Picture taken on photosensitive paper by artist Wanda Tuerlinckx www. wandatuerl inckx. com.

\section{$\S 4.1$ Control}

Energy is injected into the system by reverse-hysteresis control on the SEA: when the leg is compressed, the servo is rotated to aid in compression; at maximum deflection it is rotated back for extra push-off. The servo rest position is $\theta_{0} ; \theta_{\text {servo }}$ is its setpoint and $\theta_{\text {knee }}$ is the knee angle:

$$
\theta_{\text {servo }}= \begin{cases}\theta_{0} & \theta_{\text {knee }}<\theta_{\text {th }} \\ \theta_{0}+\theta_{\text {hys }} & \theta_{\text {knee }} \geq \theta_{\text {th }} \wedge \dot{\theta}_{\text {leg }} \geq 0 \\ \theta_{0}-\theta_{\text {hys }} & \theta_{\text {knee }} \geq \theta_{\text {th }} \wedge \dot{\theta}_{\text {leg }}<0\end{cases}
$$

Such a reverse hysteresis loop has been shown to result in stable limitcycle behaviour for hopping robots $[81,163]$. The parameter $\theta_{\text {hys }}$ determines the amount of injected energy and thereby the amplitude of the limit cycle. This controller degree-of-freedom will be used later for phase stabilisation.

\section{$\S 4.2$ Single-hopper experiments}

Single hopper control demonstration

In order to obtain a competent robot model, first a single hopper (separate anterior or posterior part) is considered. It is a relatively simple model consisting of rigid bodies connected through rotational joints, of which most parameters (mass, length, stiffness) are known. A series of 
experiments is conducted to obtain estimates for unknowns - mainly friction coefficients in the mechanism and the ground contact. The parameters were identified in an experiment with fixed controller parameters and verified for a number of different $\theta_{\text {th }}$ and $\theta_{\text {hys }}$. The predictability of the model is very high, given the maximum observed errors of $5 \%$ in hopping frequency and $10 \%$ in leg compression (Figure 6.8).

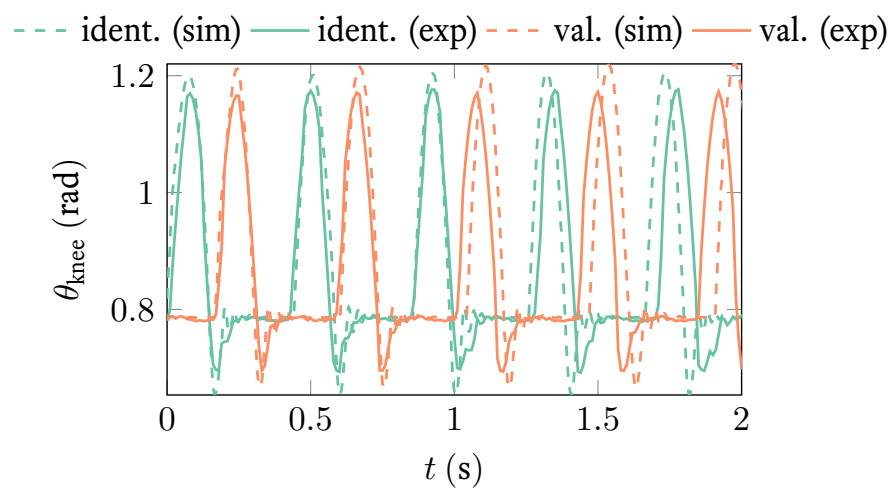

Figure 6.8 - Simulation and experiments for single hopper. The model shows good predictability: the parameters matched in the identification experiment predict a very different experiment rather well, too.

\section{$\S 5$ Results}

To investigate whether the inherent dynamics of the system can generate locomotion, the described setup is subjected to a "drop test." In these tests, the cheetah is released from a small height $(3 \mathrm{~cm})$ to provide initial energy, and observed whether it shows a tendency to move forwards or backwards, for various spine configurations.

The spine joint was moved in steps of $3 \mathrm{~cm}$, measured from the bottom middle position: $(-2,1)$ means shifted $6 \mathrm{~cm}$ to the left and $3 \mathrm{~cm}$ up. (The results summary in Figure 6.11 clearly shows all configurations.) The full results for both simulation and experiments are plotted in Figure 6.9.

\section{$\S 5.1$ Natural dynamics}

In all of the experiments, the cheetah shows a tendency to move in the direction of the spine joint: all solid lines of Figure 6.9 (spine joint on the right) have a positive velocity; dashed lines (spine joint to the left) a negative velocity. It also seems that the more "extreme" configurations, with 


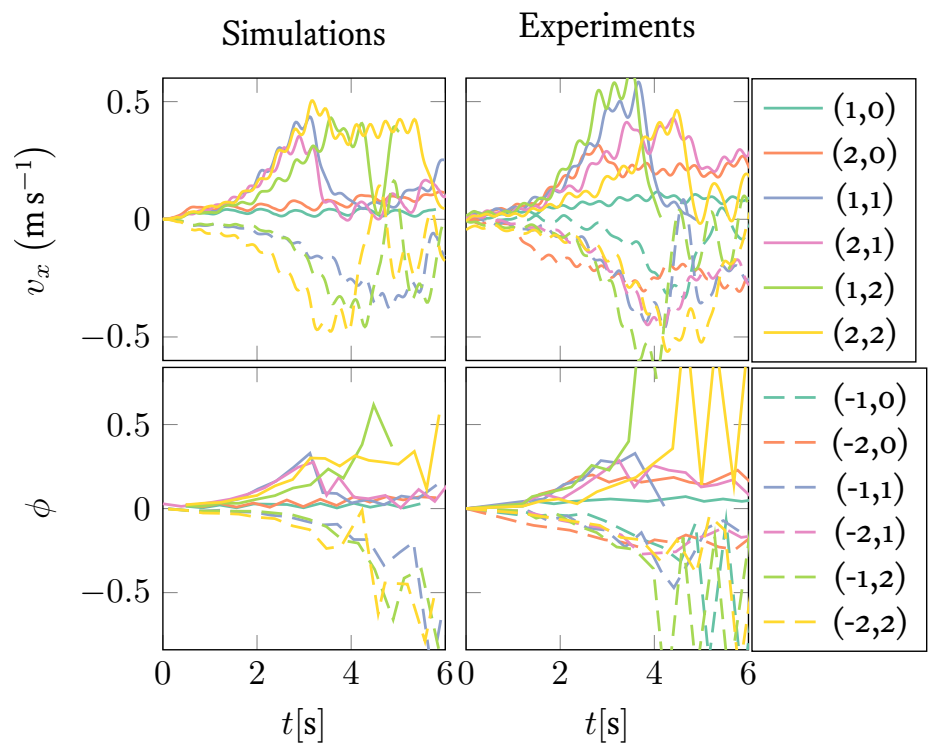

An interactive version of this plot is easily accessible at the linked site (qr code or http://freeqr.eu/bmc).

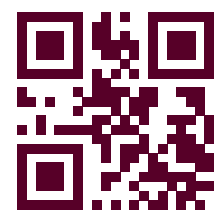

Figure 6.9 - Simulation and experimental results for the full cheetah model (without stabilising controller). The phase difference is defined as $\left(t_{\text {touchdown, } \mathrm{A}}-t_{\text {touchdown, } \mathrm{P}}\right) / T$ : a positive value means the front leg lags behind the rear leg. It is a very full plot, but similarities between simulations and experiments and the tendencies shown qualitatively in Figure 6.11 can be identified. 
the spine farthest removed form the CoM-especially $( \pm 2,+2)$ - start to move forwards or backwards sooner, and move faster, than the more symmetric configurations*. This is also illustrated by the phase difference $\phi$ between anterior and posterior body, defined as the normalised touchdown time difference $\left(t_{\text {touchdown,A }}-t_{\text {touchdown, } \mathrm{P}}\right) / T$ between front and rear feet: in the more "conservative" configurations, the legs tend to hop in phase for a long while, until the asymmetry finally achieves a phase difference and forward velocity.

It even appears that the forward speed and the phase difference are highly correlated: all configurations, once out of balance, show a rapid mutual increase in phase difference and forward speed. Unfortunately, the running behaviour is not stable for all configurations: in some, the phase difference keeps increasing until the cheetah stumbles over its own feet. After that, some configurations are able to recover and restart, whilst others lapse into unproductive behaviour like hopping-on-thespot. However, it can be concluded that the inherent dynamics indeed support graceful locomotion, predictable on the basis of the spine configuration. The chaotic or unstable behaviour, which also causes mirrored configurations to not give exactly mirrored results, may be stabilised by a simple controller.

\section{$\S 5.2$ Phase stabilisation control}

Based on the observation that successful locomotion is highly coupled to a certain phase difference, a controller that stabilises the phase difference could also stabilise locomotion. A phase $\phi$ of o or 1 means that the cheetah is pronking on the spot; a phase of $\phi=0.15$, the value observed in the stable experiments of Figure 6.9, corresponds to a bounding gait.

To stabilise $\phi$ around 0.15 , we change the amount of hysteresis $\theta_{\text {hys }}$ of (6.8) per leg, as shown in Figure 6.10. Proportional gain $K$ was optimised in simulation and a value of $1 \mathrm{rad}$ was found.

The controller works as follows. If $\phi$ is too small, the hysteresis of the anterior leg is increased and that of the posterior leg decreased. More energy will be injected in the front than in the rear (see Figure 6.10a); the period of the anterior leg will increase, posterior decrease; anterior will touch down later and $\phi$ will increase. In previous work on synchronisation, we showed this strategy to be successful [47, Section IV].

\section{§5.3 Successful morphological computation}

With the phase stabilisation controller in place, all configurations achieve sustained locomotion. The behaviour as observed in the uncontrolled

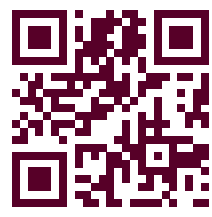

Synchronisation of two hoppers experiments remains the same with the controller in place: locomotion in the direction of the spine joint and faster locomotion for more extreme 


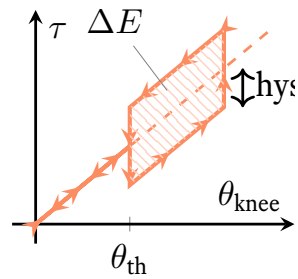

(a) The hysteresis controller of each leg generates an extra control input: the amount of hysteresis ("hys") determines the amount of injected energy.

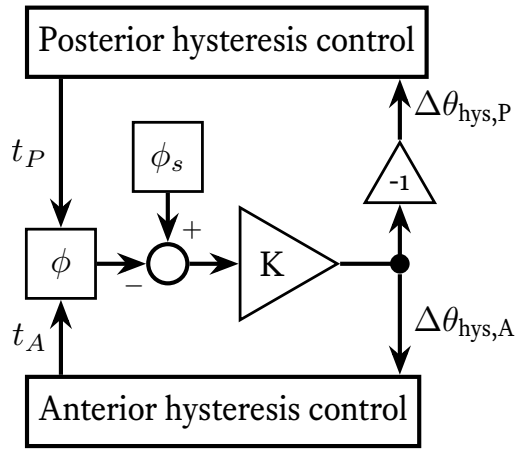

(b) Phase stabilisation: the phase $\phi$ is determined based on the touchdown times of both legs. The deviation from the desired difference $\phi_{s}$ is used to inject less or more energy in the anterior and posterior parts.

Figure 6.10 - Hysteresis control and phase stabilisation for the cheetah model, according to [47, Section IV].

configurations. This indicates that the locomotion is indeed the result of natural dynamics: the controller merely stabilises those configurations that are not inherently stable. To validate this: with the spine joint in the centre, or a rigid spine "joint," but with the controller active, the model does not show locomotion. Figure 6.11 summarises the results in one readable picture: direction, speed and (inherent) stability, caused by the single asymmetry in the robot: the spine joint's location. View a movie and plots of $v$ and $\phi$ of the running cheetah at http://freeqr .eu/bmc. 


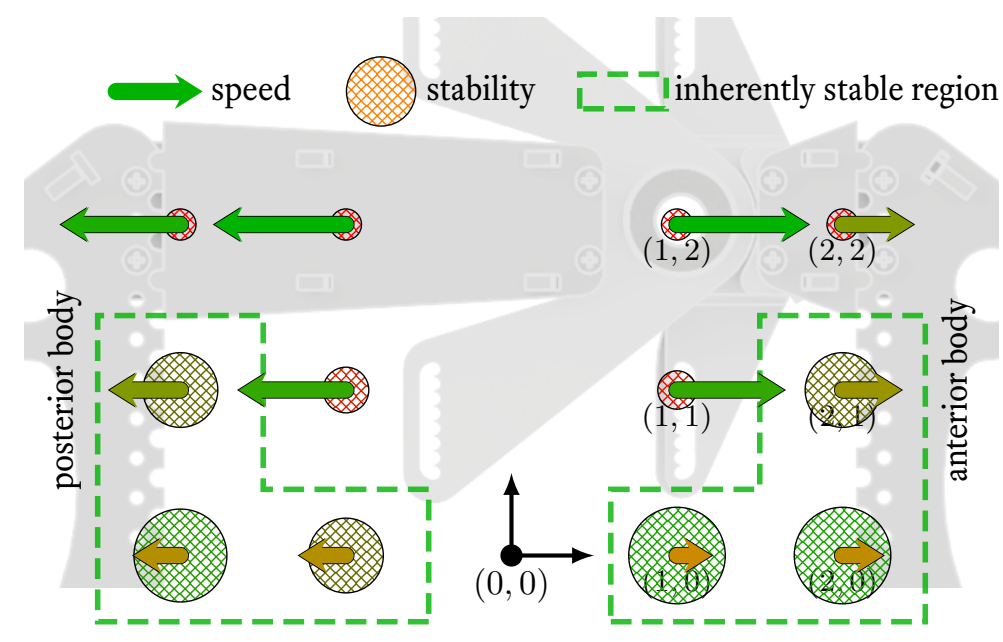

Figure 6.11 - Results of the experimental cheetah setup (without stabilisation control). The overview clearly shows that more conservative spine locations (low, between the CoMs of the two hoppers) lead to low speeds, but stable locomotion. More "extreme" configurations give rise to high speeds, but the locomotion is not very stable. With the controller of Figure 6.10, all these configurations can be stabilised. Note that the lateral location of the spine joint determines the direction of locomotion. 


\section{$\S 6$ Conclusion}

The presented research concerned morphological computation by the spine in a cheetah-inspired robot. We have argued that, when doing biomimetic morphological computation in a highly dynamic fashion, one should mimic the dynamics - the result of biology's morphological computation-rather than the morphology directly.

Video analysis of a running cheetah has shown that the spine, though being a complicated set of vertebrae and muscles, generates a motion that can be approximated very well by a simple rotational joint placed in the right position. Furthermore, inverse dynamics indicated that much of the spine's effort can be supplied by a passive torsional spring.

These two observed principles were applied on a robot that is fully symmetric, save the spine joint; the only control being energy injection. There is of course much more to a cheetah's spine than a rotation; and to a cheetah's legs than an energy pump. However, we have shown that only this is already enough to lead to locomotion; and a little stabilisation of these natural dynamics is enough for sustained running.

We hope to achieve two things with sharing this research. Firstly, to inspire quadrupedal robot designers who have not yet considered a spine

Simulation and experiment of the cheetah robot to do so; and secondly, more importantly, to show that studying and copying nature's dynamics to a biomimetic or bio-inspired robot-rather than the size, shape or structure - can lead to desired inherent dynamics and thus greatly simplify control.

This is biomimetic morphological computation. 


\section{Chapter 7}

\section{Robird}

Ever since the start of aviation, birds and aeroplanes have posed a mutual risk: birds are killed when struck by aircraft, but in return, bird strikes cause billions of damage to the aviation industry. Airports employ bird control methods such as audiovisual deterrents, weapons and chemicals to relocate, suffocate or otherwise terminate the birds. Whilst the latter methods work, they are ethically questionable. The problem of audiovisual deterrents (noise, lasers) is that they quickly lose effectiveness due to habituation.

The approach that works consistently is the use of predator birds, to scare off the prey birds and permanently relocate them away from runways. However, the predators themselves cannot be precisely controlled and in turn pose a threat to aeroplanes.

The "Robird" is a robotic bird mimicking a peregrine falcon: in appearance, but also in weight $(730 \mathrm{~g})$, size ( $112 \mathrm{~cm}$ wingspan) and flying speed $\left(16 \mathrm{~m} \mathrm{~s}^{-1}\right)$. Most importantly: the Robird is a flapping-wing aerial vehicle, mimicking the method of flight of the real falcon. The similarity in appearance and dynamics makes that other birds cannot distinguish real falcons from the Robird. This allows using the Robird for effective and nature-friendly bird control.

From a research perspective, the Robird is most interesting: much is still unknown about how it flies. Challenges are in understanding the (aero)dynamics and improving its autonomy.

This chapter will be published in the spring 2017 issue of the Robotics and Automation Magazine. 


\section{$\S 1$ Introduction}

Homo universalis Leonardo da Vinci dreamed of bird-like flying machines in his "Codex on the Flight of Birds" [158]. Failing to make those work, we have since switched to fixed-wing aircraft that perform admirably well - until they come into contact with an actual bird. Worldwide, bird strikes lead to annual damages in the billions, with a risk of plane crashes.

The danger that aviation poses to birds and, in turn, to itself, necessitates relocation of birds around airports. Conventional bird control methods include audiovisual deterrents (like scarecrows, lasers, noise), weapons and even chemicals [36]. However, habituation causes the audiovisual deterrents to quickly lose effectiveness. The more violent, ethically questionable approaches meet resistance from environmental agencies and the public in general.

One very effective natural approach is the use of birds of prey, such as falcons or eagles. Fear of these natural enemies is bred into the birds that require relocating; the approach does not lose effectiveness [36]. However, the birds of prey themselves take the place of the original birds, possibly colliding with the aeroplanes. Aside from this risk, they need to rest frequently and are by nature nocturnal, which limits their availability.

Fixed-wing or quadrotor Remotely-Piloted Aircraft Systems (RPAS) have the short-term effectiveness of scattering birds, but also suffer from habituation. Better results have been obtained with fixed-wing RPAS that look like predator birds, although actual birds of prey are not fixed-wing: they use flapping flight when hunting, so results may be improved by using an ornithopter capable of flapping and soaring [10, 90].

"Robirds" are robotic birds that closely resemble natural birds, both in appearance and in flight behaviour: they fly by flapping their wings, can soar, and have speed and wing frequencies similar to a real bird. The peregrine falcon model is capable of flying up to $60 \mathrm{~km} \mathrm{~h}^{-1}\left(16 \mathrm{~m} \mathrm{~s}^{-1}\right)$ and has the same effect on prey birds as a real peregrine falcon. Moreover, its only downtime is the changing of batteries and it can be controlled to stay off the runways. It is a perfect nature-inspired and nature-friendly means of bird control for aviation safety.

\section{§1.1 Research perspective}

The first Peregrine Falcon Robird was painstakingly put together by a falconer and model aeroplane enthusiast, in a shed in his backyard [9]. Through his experience with model aeroplanes and the study of his birds, he managed to make a working prototype by trial-and-error. This presents us with a unique opportunity: usually in engineering research, we design a device that should work theoretically and subsequently spend 


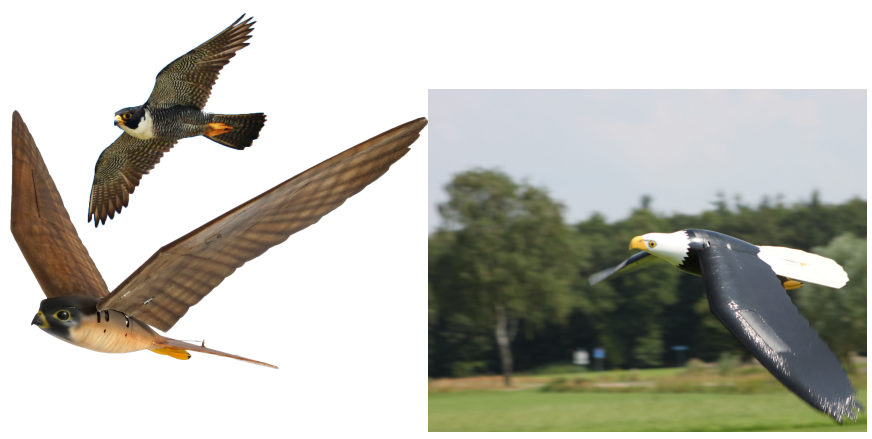

(a) peregrine falcon

(b) bald eagle

Figure 7.1 - The two Robird models. Inset of (a): an actual peregrine falcon. (Julien Baudat-Franceschi, IBC 178643. Accessible at ibc.lynxeds.com/node/178643)

much time and effort trying to make it work. Here, it is the opposite: we have a working system, but no real insight yet into how it works!

The Robird offers a myriad of research opportunities. First and foremost: how does it fly? What is the unique combination of dynamics and aerodynamics that leads to its high-performing flight? Secondly, the engineering challenge of making it better: fly longer on a battery, become self-stabilising, add autopilot capabilities, et cetera. Thirdly, we can study nature: how do prey birds react on the robotic counterpart of their natural enemy? And, excitingly: will studying the Robird's flight lead to insight into real bird flight?

\section{$\S 1.2$ Other ornithopters}

There exists a range of flapping-wing vehicles, or ornithopters, differing in scale and similarity to the animals they are modelled after.

Festo's SmartBird was inspired by a gull and it very closely mimics wing kinematics of a real gull [44]. It achieves a graceful flight with fully actuated wings; however, its size is far greater than its biological counterpart and its weight less, resulting in much slower flight dynamics. The bird can only fly in very light wind conditions. A bio-inspired ornithopter that focuses more on dynamic similarities in flight behaviour is the DelFly, an insect-like flapping-wing micro-aerial vehicle (MAV) that can both hover and fly up to $7 \mathrm{~m} \mathrm{~s}^{-1}$ [32]. Its wings are driven by a single spar, relying on the wing's flexibility for proper wing motion. Similarly but on a different scale, Harvard's Robobee mimicks the flight of a bee. It has the same size, achieving dynamics and aerodynamics comparable 
to the honeybee [168]. Also driven by a single spar, its shoulder has a passive hinge for tilting of the wings.

Like the Robobee, the Robird is matching dynamics and aerodynamics to its biological inspiration in order to achieve similar performance. It combines two spars with proper wing flexibility to achieve the required flapping motion. The Robird is the first ornithopter to do this in the weight and speed range of (predator) birds.

\section{$\S 1.3$ Peregrine Falcon and Bald Eagle}

There are currently two Robird models: the peregrine falcon and the bald eagle (see Figure 7.1). They can reach speeds of 60 and $90 \mathrm{~km} \mathrm{~h}^{-1}(16$ and $\left.24 \mathrm{~m} \mathrm{~s}^{-1}\right)$, respectively, and fly in wind speeds of up to 6 Beaufort $\left(13 \mathrm{~m} \mathrm{~s}^{-1}\right)$. They stay airborne by a combination of wing and body shape, active wing pitch control, wing flexibility, and various control surfaces for stabilisation.

While the bald eagle model flies faster and can carry a payload of up to $1 \mathrm{~kg}$, it is - due to its size and weight - also slightly more unwieldy

Several videos of the bird in flight than the peregrine falcon. More importantly, the birds we are targeting at airports are preyed upon by the falcon. Therefore, our research in this chapter focuses on the peregrine falcon model. In the remainder of this chapter, we will refer to the peregrine falcon model simply as the "Robird." Table 7.1 shows some physical characteristics of the two models and a comparison to the real birds.

\begin{tabular}{lrlrl}
\hline & \multicolumn{2}{c}{ Bald eagle } & \multicolumn{2}{c}{ Peregrine falcon } \\
& Bird & Robird & Bird & Robird \\
\hline Mass & $3.0-6.3 \mathrm{~kg}$ & $2.1 \mathrm{~kg}$ & $0.6-1.3 \mathrm{~kg}$ & $0.73 \mathrm{~kg}$ \\
Body length & $70-102 \mathrm{~cm}$ & $88 \mathrm{~cm}$ & $39-50 \mathrm{~cm}$ & $56 \mathrm{~cm}$ \\
Wingspan & $1.8-2.3 \mathrm{~m}$ & $1.76 \mathrm{~m}$ & $0.95-1.15 \mathrm{~m}$ & $1.12 \mathrm{~m}$ \\
Flight speed & $15-20 \mathrm{~m} \mathrm{~s}^{-1}$ & $18 \mathrm{~m} \mathrm{~s}^{-1}$ & $18-25 \mathrm{~m} \mathrm{~s}^{-1}$ & $16 \mathrm{~m} \mathrm{~s}^{-1}$ \\
\hline
\end{tabular}

Table 7.1 - Physical characteristics of the bald eagle, peregrine falcon and their robotic counterparts [43].

In the following sections, we address the topics: system design, (mechanical and aero-) dynamics and control. 


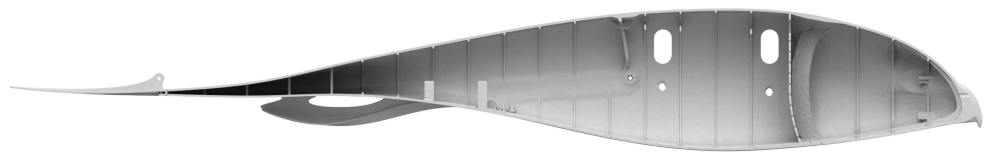

Figure 7.2 - Hull of the peregrine falcon.

\section{$\S 2$ System}

The key feature of the Robird is its flapping-wing flight. Both thrust and lift are produced by a combination of the shape and flexibility of the wings and the mechanism that not only flaps the wings (a plunging motion), but also changes their angle of attack (pitching) during the wing stroke. The wings and tail have control surfaces for stabilisation; the mechanism (Figure 7.3) and avionics for the autopilot reside inside the hull (Figure 7.2).

\section{$\S 2.1$ Wings and mechanism}

The plunging and pitching motion of the wings is realised by driving them up and down at the leading and trailing edge of the wing, with the leading edge also leading by a small phase difference $\Delta \phi \in\left[0^{\circ}, 15^{\circ}\right]$. Figure 7.3 gives a schematic overview of the mechanism. It has a latch to lock the wings in a gliding position, allowing the Robird to soar with the motor turned off. (This "glide mode" operation is explained more fully in Section 7.4.1: Flight modes.)

The wings are made of foam with a laminated resin skin, resulting in a light-weight and flexible material. While the two spars that drive the wing introduce some pitching, the flexibility combined with aerodynamic forces causes additional twisting, leading to a more favourable angle-ofattack towards the distal end of the wing. The shape, especially the sharp transition from straight to swept wing and possibly the jagged trailing edge, have turned out to be crucial for generating and subsequently shedding vortices that generate thrust and lift (Figure 7.4).

\section{$\S 2.2$ Control surfaces}

The Robird has three control surfaces: a spoiler on each wing (see Figure 7.4) and an elevator in the tail. The hinge and attachment point of the latter are just discernible in Figure 7.2. Whilst the elevator functions as a direct pitch control, steering with spoilers is rather complicated: when opened, they cause reduction of lift at that wing and induce a roll motion. At the same time their drag induces a yawing motion. Whereas the yawing initially helps to start a turn, once the Robird is rolled on its 
side the same motion now pitches the nose downwards, which leads to a loss of altitude.

The latest version of the peregrine falcon incorporates a V-shaped tail with two control surfaces that act as a combined rudder and elevator. Preliminary tests show that stability and flight performance is increased, at the cost of a slight deviation from the real bird's looks.

\section{$\S 2.3$ Avionics}

The bird is driven by a brushless DC motor (max. $112 \mathrm{~W}$ output at $11.1 \mathrm{~V}$ ) and powered by a regular, hobby-grade Electronic Speed Controller (ESC). The control surfaces are actuated using standard RC servos. All actuators are controlled by an ArduPilot Mega / Pixhawk autopilot system running on a $\mathrm{PX}_{4}$ embedded computing board [88]. The redundant and failsafe design of the software and hardware is indispensable when flying on or near airports.

\section{$\S 2.4$ Robustness}

Limited robustness is a well-known problem in ornithopters: because of the violent wing motions there is much strain on the mechanics. However, the mechanism consists of just two con rod mechanisms and the wings consist of reinforced foam, with - in the new V-tail model-no further actuators. Due to this relative simplicity of the system, the Robirds typically have an MTBF of several flight hours, which are many 10-15 min flights indeed.

\section{$\S 3$ Dynamic system model}

Studying the dynamics of the Robird is interesting in and of itself, to learn how it flies. Moreover, detailed understanding is a prerequisite for improvements to and optimisation of the system: state estimation, controller (autopilot) design and drive-train optimisation all benefit from better knowledge of the bird's dynamics.

\section{§3.1 Fixed-wing Aerodynamics}

In fixed-wing flight mode, the aerodynamics of the Robird are essentially equal to those of a glider. A glider's performance can be expressed by its glide number, the maximum lift/drag ratio [8]:

$$
(L / D)_{\max }=\frac{1}{2} \sqrt{\frac{\pi e \mathrm{AR}}{c_{D, 0}}} .
$$




\begin{tabular}{llr}
\hline$C_{D, 0}$ & 0.08 & drag coefficient $c_{L}=0$ \\
$e$ & 0.81 & Oswald span efficiency factor \\
$b$ & 1.12 & wing span $(\mathrm{m})$ \\
$S$ & 0.145 & wing surface arera $\left(\mathrm{m}^{2}\right)$ \\
\hline
\end{tabular}

Table 7.2 - Aerodynamic parameters for the glide ratio calculation of the Robird.

$\mathrm{AR}$ is the glider's aspect ratio, $b^{2} / S$, where $b$ is the total wing span and $S$ the wing area plus the area of the body directly between the wings. $c_{D, 0}$ is the drag coefficient at zero lift $\left(c_{L}=0\right)$ and $e$ is the Oswald span efficiency factor. $[131,91]$

The values for the Robird model (see Table 7.2) give a glide ratio of 8.3. This implies that it can cover a forwards distance of $8.3 \mathrm{~m}$ for every $1 \mathrm{~m}$ loss in altitude. (For comparison: the glide ratio for a glider plane is over 40 and that of a Boeing 747 is approximately 14.) There is some discrepancy between this theory and experiments with a Robird scale model, where a glide ratio of up to 18 was observed for very high Reynolds numbers. It is still unclear what causes this discrepancy, but the real falcon has a similar low glide ratio of 10 [6o]. The Robird's poor performance as a glider is partly due to the locking mechanism, which locks the wings when moving downwards, with a negative angle of attack. Also the drag coefficient $C_{D, 0}$ is very high-the wing shape of both falcon and Robird is clearly optimised for fast flapping-wing flight.

\section{§3.2 Flapping-wing aerodynamics}

Much more interesting — and challenging to characterise - is the flappingwing flight regime. There are no precise analytical results to calculate lift and thrust generation and even numerical methods can only give an approximation [64]. Qualitatively, however, flapping-wing flight works as follows.

Kinematics A 2-D cross-section of the wing (Figure 7.5) moves as follows:

$$
\left\{\begin{array}{l}
h(t)=h_{0} \sin (\omega t) \\
\theta(t)=\theta_{0} \sin (\omega t+\varphi)
\end{array}\right.
$$

where $h_{0}$ and $\theta_{0}$ are amplitudes of the plunging and pitching motion, and $\varphi$ is the phase difference between the plunging and pitching motion, determined by the phase offset between the two driving spars as shown in Figure 7.3. From these kinematics, the effective angle of attack $\alpha_{\text {eff }}$ 
can be found:

$$
\alpha_{\mathrm{eff}}(t)=\arctan \left(\frac{-\dot{h}(t)}{U_{\infty}}\right)+\theta(t)
$$

For the mid-wing cross-section of the Robird, $\theta_{0}=17.12^{\circ}$ and $h_{0}=$ $16 \mathrm{~cm}$. For a flight velocity of $14 \mathrm{~m} \mathrm{~s}^{-1}, \alpha_{\mathrm{eff}}$ is shown in Figure 7.6. The active pitching of the wing reduces the effective angle-of-attack, which is vital for stable flight.

Characteristic dimensionless numbers In flapping-wing flight, the Strouhal number is an essential characteristic dimensionless number [151]. It can be calculated by

$$
\mathrm{St}:=\frac{k h_{0}}{\pi c},
$$

where $c$ is the average chord length of the wing. $k$ is reduced flapping frequency, non-dimensionalised with $c$ and flight speed $U_{\infty}$, as in [106]:

$$
k:=\frac{\omega c}{U_{\infty}}
$$

Taylor et al. [151] have shown that the Strouhal number lies between 0.2 and 0.4 in cruising flight for 42 different species of flying animals, and even for sharks, fish and dolphins. For the Robird it lies just below 0.4.

Equation (7.6) shows that the effective angle of attack $\alpha_{\text {eff }}$ depends directly on the Strouhal number. If $\alpha_{\text {eff }}$ is in the proper range, the wing is always on the onset of dynamic stall: a Leading-Edge Vortex (LEV) is generated along the leading edge of the wing, travels in chordwise direction and is finally shed into the wake at the trailing edge. These LEVs are essential for flapping-wing flight.

$$
\alpha_{\mathrm{eff}}(t)=\arctan \left(\frac{-\dot{h}(t)}{U_{\infty}}\right)+\theta(t)=\arctan (\mathbf{S t} \pi \cos (\omega t))+\theta(t)
$$

Von Kármán vortex street In an airflow, vortices are generated and shed about stationary blunt objects, resulting a so-called Von Kármán vortex street: a series of vortices that are shed alternating on the two sides of the object, rotating clockwise above and counterclockwise below the object. The vortex street induces a velocity field at the object, resulting in an oscillating force on the object that can cause it to vibrate (e.g. conductor gallop in power lines). In the wake, the vortex street induces 
a deficit in the stream-wise velocity that is a reminiscence of the object experiencing a drag force.

In bird flight, the plunging and pitching causes the vortices to be shed such that the top and bottom vortices are switched: a reversed Von Kármán street is generated. The vortex street now causes a rearwards-pointing jet-like flow that induces a thrust force on the wing.

\section{$\S 3.3$ Dynamics}

All the aerodynamics results in the preceding section start from an observation of the wing motion, which is then used as a rigid (kinematic) input to a computational fluid dynamics (CFD) method. To truly understand the flight behaviour, the full fluid-structure interaction should be solved: in reality the wing is not a rigid input to the airflow; rather, there is an interconnection, a bi-directional interaction, between fluid dynamics and structural dynamics.

While in theory it is possible to generate a fine FEM model of the wing and connect it to a CFD model of the surrounding air - numerically solving the Navier-Stokes equations in conjunction with wing deformationthis would require enormous computation resources. The solution of this problem lies in proper simplification.

Currently, we are working on dividing the wing in a number of rigid bodies in Port-Hamiltonian Systems form, which gives them a power port for interacting with the aerodynamics. The optimal number and distribution of bodies and their parameters (mass, coupling stiffness, damping) are determined by comparing simulations with experiments in a wind tunnel. We place markers on the wing (visible in Figure 7.8) and, with a stereoscopic camera setup and a stroboscope, obtain high-frequency $3-\mathrm{D}$ wing motion at various wind speeds, flapping frequencies, and leadingtrailing edge phase differences. The Port-Hamiltonian lumped-system model is then numerically optimised to show the same motion.

Because the simulation currently uses simplified aerodynamics equations, it is still difficult to identify dynamic wing parameters from these measurements - the aerodynamic effects cannot be easily separated from the wing dynamics. One of the next steps in our research will be to place the wing in a large vacuum chamber and carry out measurements without any aerodynamic effects: this will allow us to accurately estimate the wing parameters. Comparing these measurements with the wind tunnel measurements will also allow to precisely identify the contribution of the aerodynamic effects. 


\section{$\S 3.4$ Wind tunnel measurements}

Exploiting the lateral symmetry of the Robird, a model of half the bird has been placed in a wind tunnel to measure forces on the body and to study the air flow around the beating wing $\left(3 \mathrm{~Hz}, 5^{\circ}\right)$. The forces are compared to simulation results of a very simple 2-rigid-body dynamical model, with the mass distribution of the wing lumped in a front and rear half-wing, both connected to one driving spar. This simple model cannot capture most of the higher-order dynamics or the aerodynamic forces. Figure 7.7 shows the measured forces, where $x$ points forwards (into the tunnel) and $z$ upwards (along the wing when upright). The most important experimental observation is in the plot of $F_{x}$ : the wing indeed generates a positive average thrust force.

Figure 7.8 shows a picture of smoke trail measurements, visualising the vortices around and behind the wing.

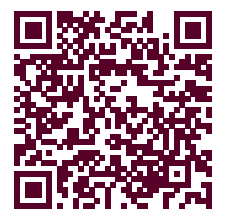

Anaglyphic 3-D videos of experiments in a wind tunnel at UT and a vacuum chamber at ESA. (Use red/cyan glasses to see the 3-D effect.)

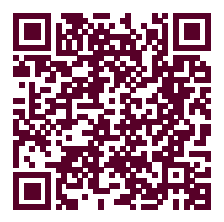

Marker tracking in wind tunnel and vacuum chamber experiments, plus simulation of a dynamic wing model. 


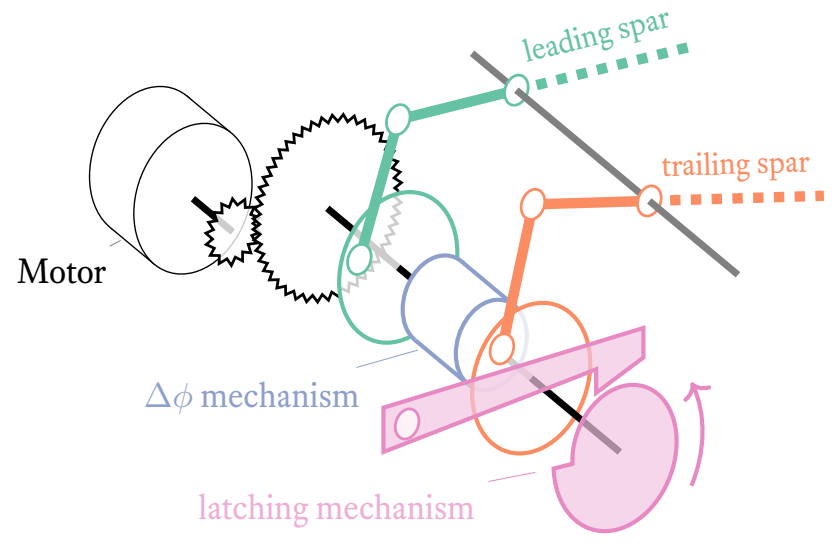

Figure 7.3 - Schematic drawing of the wing mechanism: the leading and trailing edge of the wing are driven with a phase difference $\Delta \phi \in$ $\left[0^{\circ}, 15^{\circ}\right]$. Their rotation is transformed to a flapping motion of the wing through a con rod mechanism for each driving spar. If the motor is stopped at the right time, lift pressure pushes the notch wheel against the pawl (latching mechanism), which allows the Robird to soar with the motor turned off.
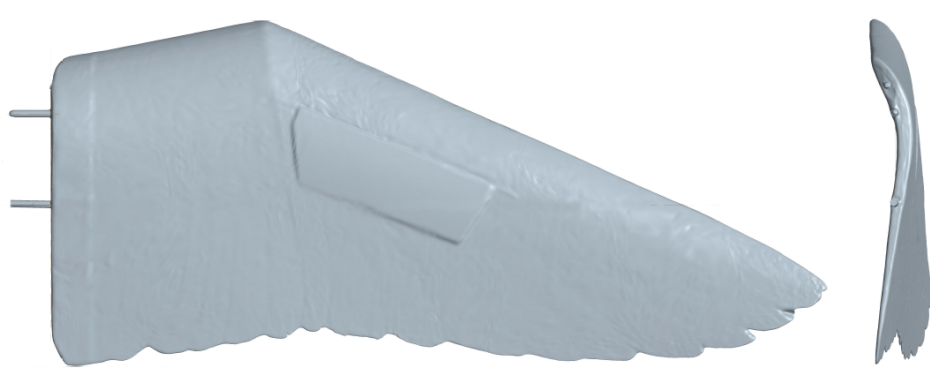

Figure 7.4 - Wing of the peregrine falcon. Note the spoiler-trapezoidal patch in the centre-used for rolling manoeuvres, and the two spars used for driving the wing. 


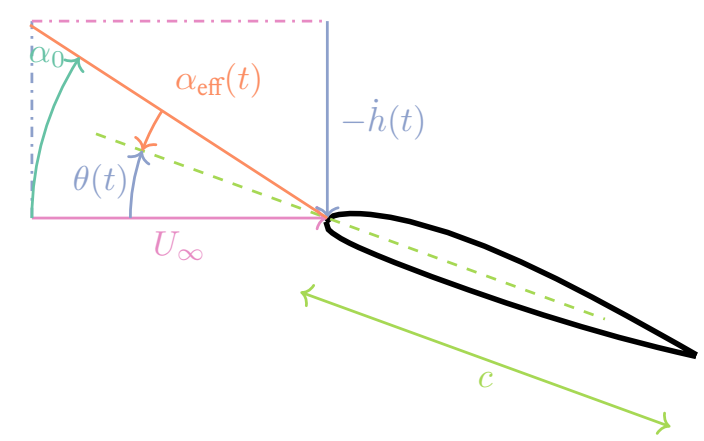

Figure 7.5 - A 2-D cross-section of the wing: the plunging velocity $(\dot{h}(t))$ and flight velocity $U_{\infty}$ result in an angle-of-attack $\alpha_{0}$, which is reduced due to pitching $(\theta(t))$ to the effective angle-of-attack $\alpha_{\text {eff }}(t)$. ( $c$ is the chord length of the wing.)

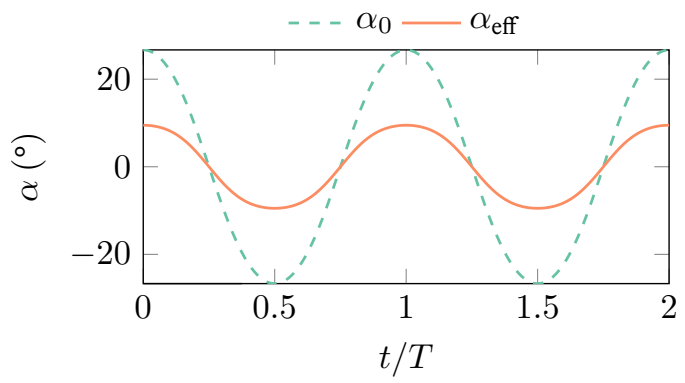

Figure 7.6 - The effective angle of attack $\alpha_{\text {eff }}$ is a result of the interplay of the plunging motion $(\dot{h}(t))$ with the free-stream (flight) velocity $U_{\infty}$ and the wing's active pitching. Without pitching, the wing would stall because of the high angle of attack $\left(\alpha_{0}\right)$. 

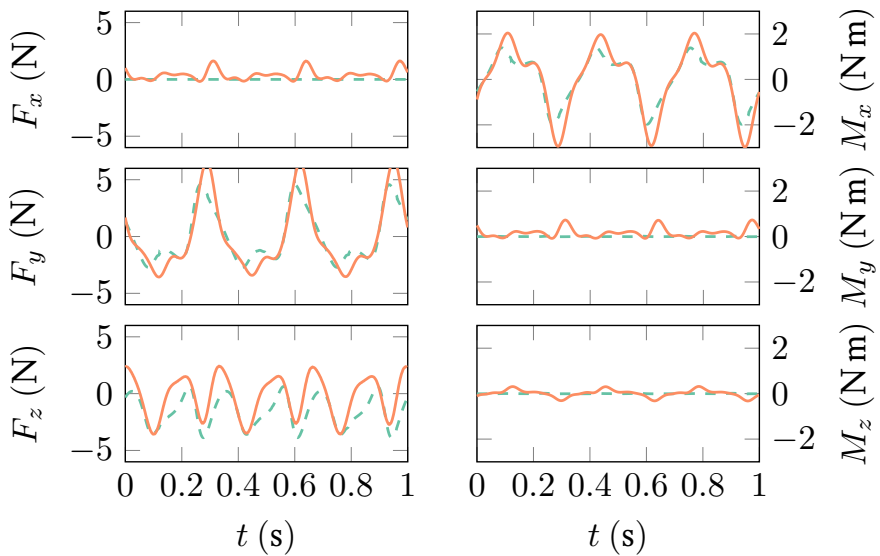

Figure 7.7 - Force measurements in the wind tunnel (solid line $-)$, compared to purely inertial forces in a simple 2-rigid-body dynamical model (dashed line -- - -). Note that $F_{x}$ is positive on average, implying that the wing generates thrust. $f=3 \mathrm{~Hz}, \Delta \phi=5^{\circ}, U_{\infty}=$ $0 \mathrm{~m} \mathrm{~s}^{-1}$

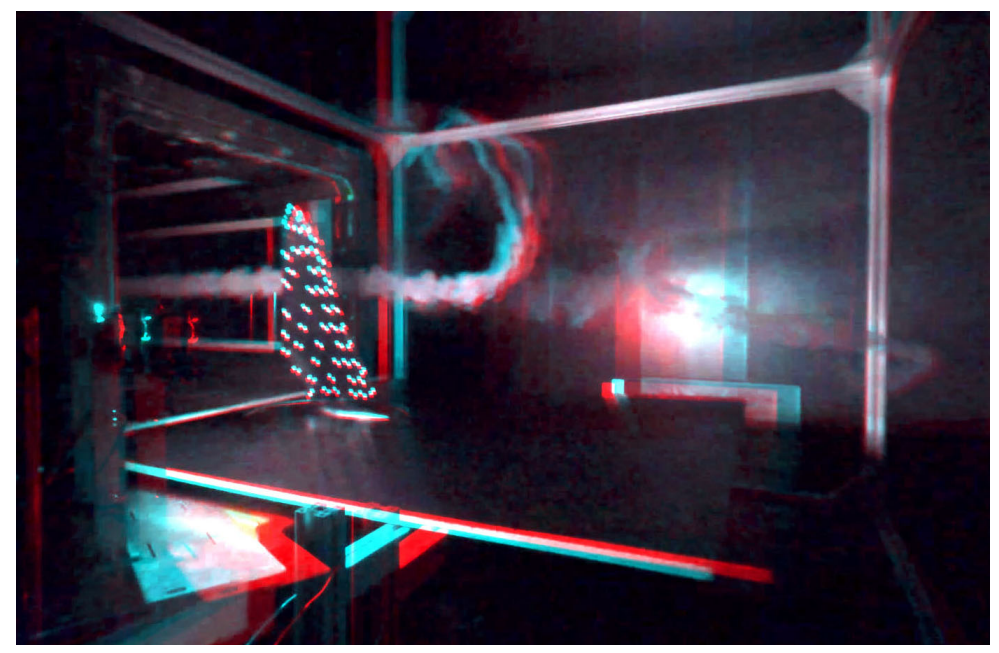

Figure 7.8 - 3-D smoke trail measurements in the wind tunnel. Use a pair of red/cyan anaglyphic glasses to see the 3-D effect. The inlet of the tunnel is on the left, with the wing positioned right in front of it. The smoke trail shows the vortex street that is generated by the wing. 


\section{$\S 4$ Control}

Although there are a few very skilled pilots who can manually fly the Robirds, the cognitive load is too extensive when flying close to runways for an entire day. An autopilot system should make flying easier, such that in the end anybody could fly the Robird—or indeed program its flight path.

When in gliding mode, the bird closely resembles a normal glider plane and the standard autopilot of the Pixhawk/APM system suffices. The challenge lies in the flapping flight and in switching between modes.

\section{§4.1 Flight modes}

As shown in Section 7.2: System (Figure 7.3), the flapping mechanism features a latch that acts as a lock for a passive gliding mode. For the latch to engage - due to aerodynamic lift pushing the notch wheel backwardsthe wings must be stopped in the right position, at a dihedral angle of approximately $20^{\circ}$ (Figure 7.9b). A gliding controller takes care of automatically engaging the lock when the throttle drops below a threshold, as depicted in Figure 7.9. The main drive shaft has an angular encoder measuring the shaft angle $\phi$; the latch can engage when $\phi \in\left[\phi_{m}, \phi_{M}\right]$.

\section{$\S 4.2$ Autopilot}

While research on a workable (aero)dynamic model of the bird in flapping flight is in progress, for stabilising control we have to rely on existing autopilot controllers offered by the Pixhawk/APM board, tuning parameters by observations of many failed flights, pilot experience and logged data analysis. Without a very skilled pilot able to save the bird whenever the controller destabilised the flight, it might have proven impossible. We are now at a point where the autopilot can successfully stabilise the system.

There are some challenges left, most of which relate to the flapping flight - with vertical accelerations of $3 \mathrm{~g}$ in amplitude and heavy pitching, even state estimation is not straightforward. The reason that the autopilot - which was designed for fixed-wing aircraft - only has to be slightly adapted for our flapping-wing system, is that the flapping frequency is relatively low, compared to smaller-scale ornithopters: $6 \mathrm{~Hz}$ is well within the range of the (aero)dynamics of fixed-wing aircraft and hence the autopilot can handle it. A full path of a flight with the autopilot in stabilising fly-by-wire mode is shown in Figure 7.10; the figure-8s in the flight path are the bird autonomously loitering. 


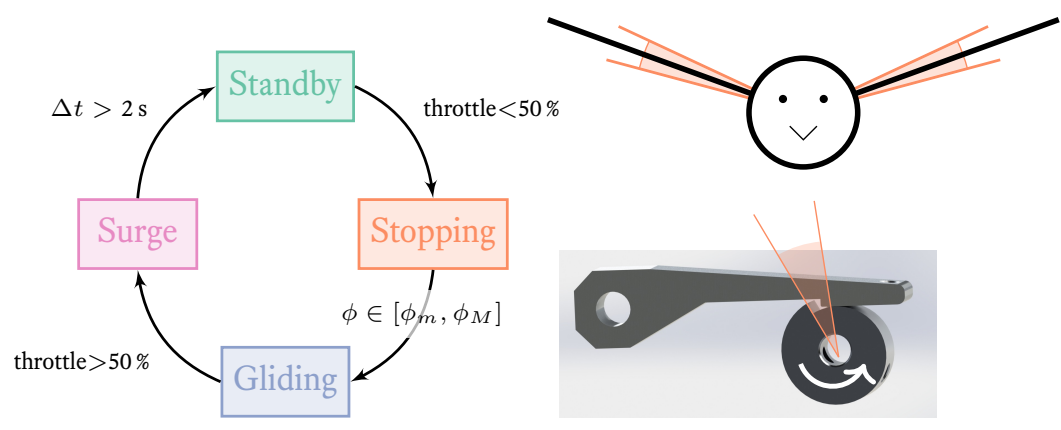

(a) Finite state machine displaying controller states and transition guards.

(b) The range wherein the The "surge" state is required to pull the bird out of gliding mode, by supplying a high throttle for a short time. lift force can engage the gliding lock. Top: dihedral angle range; bottom: drive shaft/latch range.

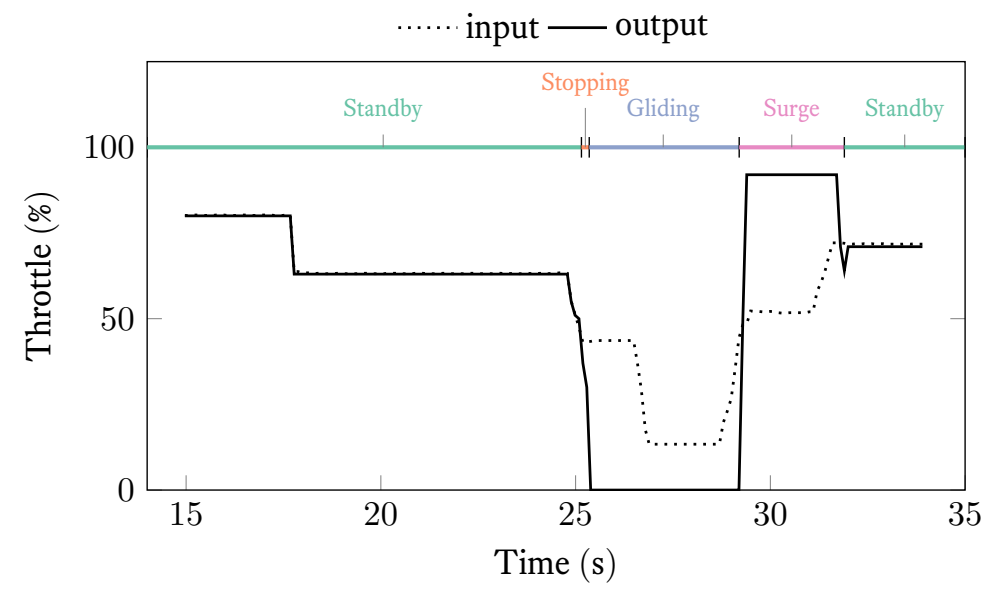

(c) Demonstration of the gliding controller. The throttle threshold is 50\%; input is the manual RC signal received from the pilot and output goes to the ESC.

Figure 7.9 - The gliding mode controller automatically engages the mechanism's passive latch when the throttle drops below a threshold. 


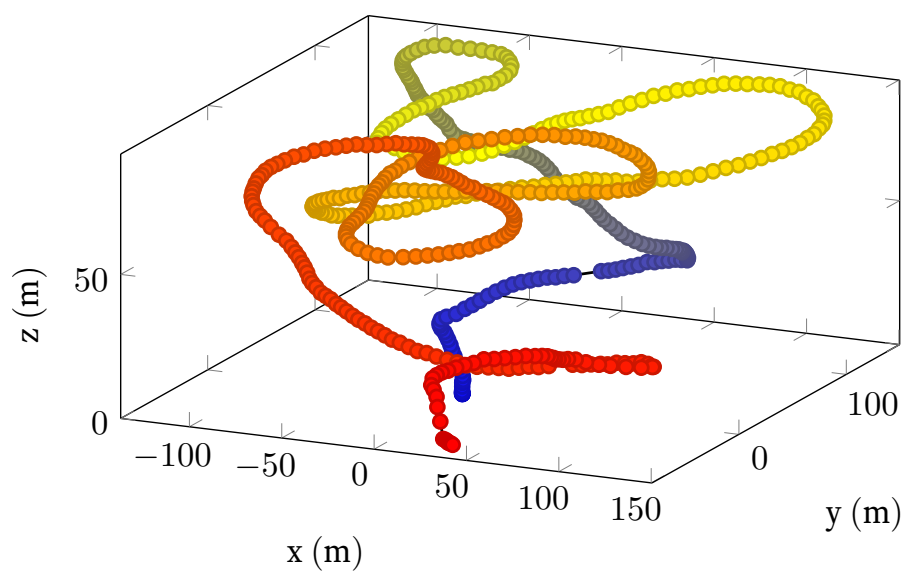

(a) Flying from blue to red.

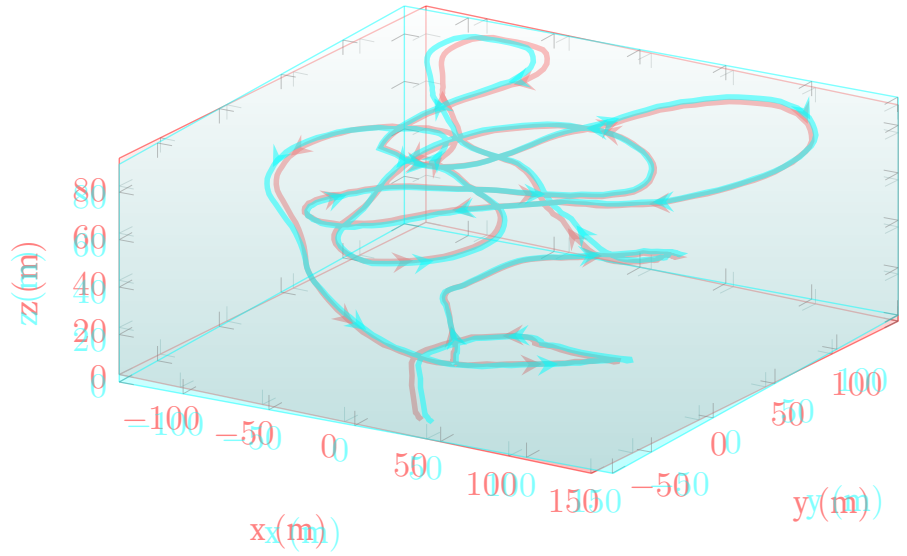

(b) Anaglyphic 3-D flight path (same as 7.10a; view with $\mathrm{red} /$ cyan glasses).

Figure 7.10 - 3-D flight path log. The bird was brought to altitude in fly-by-wire mode, then autonomously loitered around $(0,0,80)$ and $(0,0,60)((x, y, z)$ setpoint in metres) before being brought down to land by the pilot. 


\section{$\S 5$ Conclusion}

Some five hundred years after his days, Leonardo da Vinci's dreams about artificial flapping-wing flight have come true: in ornithopter toys, Robobees, kinematic seagulls and now the Robird. The Robird's similarity to the peregrine falcon allows the spin-off company Clear Flight Solutions [28] to use it for bird control, but at least equally interesting are the research opportunities.

There is a qualitative understanding of how flapping-wing flight at this scale works, and simple mechanical and aerodynamical models have been developed. The bird can be controlled in gliding and flapping-wing flight, although the robustness can be improved. Current work therefore focuses on improving the dynamic model of the bird, by designing more realistic measurement set-ups and collecting data on both aerodynamic and structural dynamical behaviour.

With this, we hope to answer questions like "How to improve the flight efficiency?" and "How to do proper, on-board state-estimation and control on such a highly-dynamic system?"-but also "Are the principles of the Robird close to how real birds fly?" and "Could we use this system to study real birds?" 


\section{Chapter 8}

\section{Conclusion}

Robotic research may be inspired by nature. The question in biomimetic design is always: "What is the proper way to do biomimetics?" Or, as put by Pfeifer, Lungarella and Iida [104]:

“ Simply copying a biological system is ... of little interest ... . Rather, the goal is to work out principles of biological systems and transfer those to robot design. "

With a running quadrupedal robot as objective, this work has focussed on the cheetah. And, rather than copying kinematics, looks, or morphology in general, the principles that were worked out are those related to high-speed, dynamic locomotion. Therefore the main, motivating research question was:

\section{“What are the important dynamic functions in a running cheetah? "}

To arrive at an answer, the following questions were addressed too:

1. How can these functions be identified?

2. Can they be identified in a quantitative manner?

3. What tools and methods are needed to describe them?

4. How can the essential functions be synthesised into the design of a robot?

In this chapter we answer some of these questions, based on the work presented in this thesis. The significance of these answers - or in other words, the implications for and contribution to the research field-will be discussed as well. Finally, as is their wont, the answers raise even more questions. Those are the topic for further discussion and research. 


\section{$\S 1$ The answers}

Our way towards a working quadruped, towards a running cheetah robot, was not one of separate mechanic and controller design. Instead, a holistic view of the complete system was first developed, before starting on implementation of each sub-system. The reason for this approach is the fact that in dynamic locomotion, the dynamics of the mechanical system interact with those of the controller; together they shape the behaviour. Rather than compensating for the physical system dynamics and trying to force them into some desired behaviour, the two complement each other: this is dynamic morphological computation.

The resulting dynamics are a combination of various oscillations or other resonance-based phenomena. Energy flows back and forth between physical storage elements - springs and masses - directed by the kinematic structure, but also between the physical system and the control algorithms. An energy-based view for both the controller and system dynamics gives insight into these energy flows. More importantly, there is no conceptual distinction between the controller and physical system: both are characterised by energy storage elements and their interconnection. Moving from design to implementation, there is a shifting boundary between controller and physical system: the choice of what behaviour is implemented where is not dictated by the formulation and becomes a free design choice.

\section{$\S 1.1$ The cheetah robot}

In order to identify the dynamic principles of the running cheetah, a slow-motion video of the running cheetah was analysed. Tracking the position of the anterior and posterior bodies, the kinematics were first identified. With inverse dynamics, the forces that act on these bodies were found. However, a cheetah robot is not equipped with full actuation on the bodies. In order to do something with the information, it should be fitted to an abstract (dynamic) model. We focus on the spine, hence the split in anterior and posterior body. This focus also gave a way to interpret the results: looking at the elastic behaviour of the cheetah's spine.

To isolate the spine's contribution to locomotion, the entire locomotion behaviour should originate from the spine. The legs, therefore, only need to inject energy to keep the motion going. There still needs to be push-off and ground clearance, so the legs in this work were modelled, designed and built as simple "hoppers." They really do nothing but bounce up and down, leaving all other locomotion dynamics to the spine. Of course, the spine does this by interacting with the legs, too: there is a combined oscillation or resonance. Therefore, the legs were designed 
such that they allowed oscillation, by being compliant and losing little energy in ground impact.

Although the combination of hoppers and a fully elastic spine showed successful locomotion, the robustness and stability were limited. To improve the behaviour, to exercise more control over the gait, the synchronisation of oscillators was studied, resulting a very generic, geometric description of the synchronisation phenomenon.

With all three conceptual aspects of dynamic locomotion in placethe spine, the hoppers, and synchronisation between them - the final cheetah robot was designed. The spine has been simplified to a rotational joint with compliance that can easily be implemented mechanically. The new legs, based on Evans' mechanism, combine energy-efficiency of the first hopper model, with more mechanical robustness and the ability to inject more energy. This cheetah robot shows locomotion induced by the spine, stabilised by a synchronisation controller between the legs. The cheetah robot exhibits successful energy-based, biomimetic, dynamic morphological computation.

To give explicit answers to the biomimetic cheetah research questions:

1. How can these functions be identified?

By observing a running cheetah and choosing some aspects.

2. Can they be identified in a quantitative manner?

Yes: a video of a running cheetah may be analysed to give kinematic and dynamic information of the running motion.

3. What tools and methods are needed to describe them?

To interpret the information, it needs to be cast into a framework. Here, an energy-based and geometric framework was chosen.

4. How can the essential functions be synthesised into the design of a robot? By abstracting the dynamic behaviour into simple formulations, such as rotational springs and energy-efficient hoppers.

It is important to note that there are multiple arbitrary choices in these answers: the aspects that were studied, the specific video material, the theoretical framework and fabrication methods. However, with these choices, we have arrived at an answer for the main research question; an answer that is backed by a cheetah robot that runs due to biomimetic morphological computation by the spine:

“What are the important dynamic functions in a running cheetah? "

A mutually synchronised hopping anterior and posterior body, connected by a compliant spine. 


\section{§2 The implications}

The motivation for the research presented in this thesis was the development of a cheetah robot. However, that was not its purpose or its goal. The purpose, as stated in the introduction, was

" to bring together the Port-Hamiltonian System theory and the field of morphological computation. "

And the goal, the envisioned result:

" not only a running quadrupedal robot, but also the methodology that enabled the design and realisation of this robot. "

In the following sections, the insights, implications, contributions and limitations of the work are discussed. They are discussed on three levels, for three fields: quadrupedal robots, biomimetic robots and energy-based robots.

\section{§2.1 Quadrupedal robots}

The spine of a real cheetah was shown to exhibit compliant behaviour: from a video analysis of the dynamics, a large part of its force can be supplied by a passive compliant element. It was shown that a fully compliant spine, modelled as a spring with a Remote Centre of Compliance, is capable of inducing locomotion in an otherwise fully symmetric model. A simplified version, the rotational spine of the final cheetah robot, could be made to achieve the same.

It cannot be argued that a spine is necessary for locomotion in quadrupeds, since many of the existing quadrupedal robots do not have a spine. Nor can it be argued that the legs are required to make some kind of controlled running motion, since in this work, they were simple hoppers that do not actively pursue locomotion.

However, the work presented in this thesis does show that it is worthwhile to look at the addition of a spine to quadrupedal robots, because it can improve the dynamics of locomotion-it can make them better resemble the dynamics of a real galloping cheetah. Furthermore, this spine does not have to be a full artificial spinal column, or a rotational or translational joint somewhere in the middle of the body. Instead, it can be a compliance, with geometric properties that determine the emergent locomotion behaviour. Hopefully, more researchers can benefit from these lessons and more quadrupedal robots will feature a spine in the future. 
It is important to note some important limitations to the cheetah robot of this thesis, especially in light of the original proposal, which called for a "Fast-running, Energy-Efficient Quadruped" (see Appendix A).

Fast-running Both cheetah models definitely run, based on various definitions of running (Centre of Mass trajectory, flight phase). What exactly "fast" means can be a topic for discussion, but comparing the speed of the final cheetah robot with the speed of the real cheetah gives the following:

\begin{tabular}{lccc}
\hline & $v\left(\mathrm{~m} \mathrm{~s}^{-1}\right)$ & $L_{\text {body }}(\mathrm{m})$ & $v / L_{\text {body }}\left(\mathrm{s}^{-1}\right)$ \\
\hline Real cheetah & 25 & 1.3 & 19 \\
Cheetah robot & 0.3 & 0.3 & 1
\end{tabular}

Clearly, the normalised velocity (in body lengths per second) of our robot is still a far cry from the real cheetah's.

Energy-Efficient The premise of energy-efficiency was that, if the mechanical system is such that its natural dynamics show locomotion, the actuator or controller will have to spend little energy to keep this going, opposed to when the controller has to force the robot into some kind of behaviour. Although the energy-efficiency of the hoppers in isolation was studied and optimised, there are no measurements yet on the efficiency of the complete robot.

Ongoing work on the system - see also the future work in Section 8.3: The new questions - indicates a Mechanical Cost of Transport, a non-dimensional measure for efficiency, of approximately 0.3 , which is a bit better than some (Bigdog: 15, MITCheetah: 0.5 [130]) and a bit worse than others (ETH Monopod: 0.1 [55]). It is actually close to that of the cheetah $(0.3,[154])$ * $^{*}$

Quadruped All the cheetah models presented in this thesis are restricted to the sagittal plane, to focus on the locomotion behaviour without dealing with lateral stability. Therefore, technically, they are twolegged. However, the fact that the legs are separated by the body makes the dynamic behaviour similar to that of a bounding quadruped. Theoretically, it should be possible to duplicate the planar model, connect the two together and have a working quadruped.

\section{$\S 2.2$ Biomimetic robots}

Much of the existing work on morphological computation is based on kinematics. In this work, this has been extended to dynamic morphological computation. The system behaviour is described in terms of desired dynamics, which are a result of internal resonance and energy flowing between storage elements, both in the physical model and in the control

* It must be noted that the Total Cost of Transport may turn out to be higher, depending on the efficiency of the actuation.

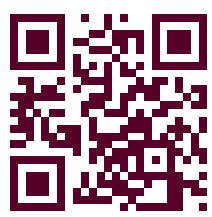

New result: four-legged cheetah robot 
algorithms. The combination of Port-Hamiltonian System theory and morphological computation, both explicitly describing the interconnection of (physical) storage elements, gave insight into the dynamics and energy flows. The graphical expression in bond graphs visualises the interconnection structure, which leads to more intuitive understanding of the energy-routing control approaches: the routes can literally be seen in the graphically modelled dynamics.

Using this approach, it was shown that new lessons can be learned from nature by looking at it in a different way: by looking at the dynamics, by identifying the storage elements and their interconnection, by studying the geometric aspects of the spine. This approach can be valuable to any research into biomimetic robots, whenever the desired behaviour is a dynamic behaviour.

\section{$\S 2.3$ Energy-aware robots}

The energy-based view of modelling and control is not new (see e.g. [94, 123]), but many practical robotic systems are still operated with traditional control methods. In Chapter 2: Energy in robotics it was shown how to apply many of the abstract principles from control theory to real robotic systems. Important results from the mathematical community become tangible to robotics researchers. The idea of passivity and energy budgets, with its practical implementation in the actuator equipped with a virtual energy tank (Section 2.2: Energy in controlled physical systems), can be very valuable to the field of robotics, especially when physical human-robot interaction is concerned. (See Appendix $C$ for a not-yet published paper on this subject.) However, in reality, friction and other non-ideal aspects make control over energy difficult, or deteriorate performance when combined with traditional control methods. Even so, we have made steps to apply the shaping, measuring, directing and controlling of energy in real robotic systems.

The synchronisation of limit cycles that was investigated for gait stabilisation led to a novel look at the phenomenon of synchronisation. A new, coordinate-free, geometric definition of "synchronisation" was developed. It allows synchronisation of arbitrary periodic systems, by describing topological connections between arbitrary limit cycles. In light of the energy-based analysis and design perspective, it was shown how to achieve power-continuous synchronisation, by modulating the energy flow between two systems. The practical applicability of the method depends very much on the systems in question; controllability aspects were not investigated. However, the synchronisation method was successfully applied on the cheetah robot to stabilise its gait. 


\section{§3 The new questions}

The answers presented in the preceding sections are not the definitive answers. They are the result of a specific way of looking at the problem, using a specific formulation, and working towards a specific goal. Some limitations were already indicated; they give rise to new questions. We are already working towards answers to some of these questions in future research. Others are topic for further discussion.

\section{$\S 3.1$ Quadrupedal robots}

Spine The spine in the final cheetah robot is a fixed rotational joint. It would be interesting to make the location of the joint not only adjustable, but controllable: considering its direct influence on locomotion, the robot could be steered using this extra control input. Furthermore, the robot is currently being extended to a full quadruped, by duplicating the planar version. Questions in this research are: how should the two halves be coupled? Can a compliant spine passively provide lateral stability? Can the spine also facilitate turning, if its geometric properties are controlled?

Legs The current legs are designed as simple hoppers that only inject energy by bouncing up and down. While this was required to show that a spine can induce locomotion by itself, the performance could be improved if the legs also actively contribute to this. Currently, a novel leg mechanism is studied that allows a continuous range from hopping (feet moving up and down) to active locomotion (feet moving in a circular trajectory). It must be studied whether this allows for "directed energy injection," shaping the internal motion of the cheetah in such a way that the energy injected by the feet contributing to forward velocity is maximised.

Synchronisation The definition of synchronisation is not enough to practically apply it. The control aspects must be further studied: can the system always be steered in the horizontal and vertical tangent planes? Can synchronisation still be achieved if the control distribution $g(x)$ is really state-dependent, or if the system is not fully actuated?

Furthermore, the practical applicability of the energy-routing control methods presented in the examples must be studied. For example, changing stiffness in real robots may be achieved using Variable Stiffness Actuators (VSAs). Is it possible to equip the hoppers with VSAs and use them for synchronisation of the various oscillations in the system?

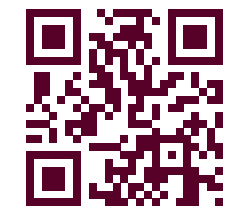

First simulation experiments with the new hopper design 


\section{$\S 3.2$ Biomimetic robots}

In Chapter 7: Robird, a new biomimetic flying robot was introduced. The Robird can fly, but it is not yet clear how it does this, exactly. In the near future, we want to apply the energy-based view to this ornithopter: to describe the aero- and structural dynamics in a port-based way, to improve the efficiency, and to understand its flight a little better. The phase difference in the driving of the wings, that is currently enforced by kinematic constraints, may be achieved dynamically: by using the synchronisation methods. This may result in an ornithopter with a dynamically varying phase difference, always flying at the optimal Strouhal number.

Next, the kinematic coupling between the left and right wing may be released in a similar way, to allow individual control over both wings. This should enable steering the Robird purely by the wings, thereby obsoleting the flaps, spoilers and rudders that are currently required.

\section{$\S 3.3$ Energy-aware robots}

Further work has already been done on the idea of energy budgets and an actuator with an embedded, virtual energy tank. This "energy-aware actuator" leads to guaranteed passivity and thereby stability and safety, regardless of the control algorithms used. Still, the most challenging question has not been answered yet: how to determine the energy budget?

In human-robot interaction, safety is of the utmost importance. Control over the energy exchanged in the interaction can greatly improve the safety. The extension of the energy-aware actuator to this field requires answers to the following questions:

1. Can the energy exchange between robot and environment (human) be measured?

2. How can this energy exchange be controlled, or limited?

3. In a rehabilitation robot, the mass of the robot must often be compensated using an admittance controller. Can this be done in a safe, energy-consistent way?

Finally, the "holistic view," where the boundary between control algorithm and physical system is flexible, will be applied to more systems. A specific example is the use of VSAs in a robot arm with an impedance control strategy: part of the desired dynamics - a certain workspace stiffness - may be achieved by the VSAs, in a safe and energy-efficient manner. 


\section{$\S 4$ Conclusion}

In this thesis, we have investigated biomimetic and energy-based robotics. The motivation was a running quadruped, a cheetah robot. A methodology for biomimetic, energy-based, dynamic morphological computation was developed and has resulted in a robot that runs. The lessons learned from the cheetah may be applied by other researchers working on quadrupedal robots and the methodology itself can be used for other biomimetic robots. More broadly, the energy-based perspective on robotics has led to new tools, new insights and new ideas to use in robotics research. 
Chapter 8

Conclusion 


\section{References}

[1] J.A. Acebrón et al. "The Kuramoto model: A simple paradigm for synchronization phenomena". In: Reviews of Modern Physics 77.1 (2005), pp. 137-185. DOI: 10.1103/RevModPhys .77.137.

[2] AMS ag. AS5045-12 bit rotary position sensor - AMS. 2014. URL: http://www.ams.com/eng/Products/Position-Sensors/ Magnetic-Rotary-Position-Sensors / AS5045 (visited on 01/09/2014).

[3] Maxon Motor Ag. RE-max 29, D29 mm, Graphite Brushes, 22 Watt, with terminals. 2014. URL: http : / / www . maxonmotor . com/maxon/view/product/226806 (visited on 01/09/2014).

[4] B. K. Ahlborn and R. W. Blake. "Walking and running at resonance". In: Zoology (Jena) 105.2 (2002), pp. 165-174.

[5] R. Alami et al. "Safe and dependable physical human-robot interaction in anthropic domains: State of the art and challenges". In: Intelligent Robots and Systems, 2006 IEEE/RSJ International Conference on. Oct. 2006, pp. 1-16. DOI: 10.1109/IROS. 2006. 6936985.

[6] Alin Albu-Schäffer et al. "Anthropomorphic Soft Robotics From Torque Control to Variable Intrinsic Compliance". English. In: Robotics Research. Ed. by Cédric Pradalier, Roland Siegwart and Gerhard Hirzinger. Vol. 7o. Springer Tracts in Advanced Robotics. Springer Berlin Heidelberg, 2011, pp. 185-207. IS BN : 978-3-642-19456-6. DOI: 10.1007/978-3-642-19457-3_12.

[7] RM Alexander and HC Bennet-Clark. "Storage of elastic strain energy in muscle and other tissues". In: Nature 265.5590 (1977), pp. 114-117.

[8] John David Anderson. Introduction to flight. 7 th ed. ISBN13 978o073380247. McGraw-Hill Boston, 2011. 
[9] ANP. "Vogels op Schiphol opgepast voor de 'Horck"'. In: Trouw (Apr. 2008). Dutch newspaper interview with inventor Robert Musters. URL: http : / / www . trouw . nl / tr / nl / 4332 / Groen/article/detail/1242931/2008/04/11/Vogels-opSchiphol-opgepast-voor-de-rsquo-Horck-rsquo.dhtml.

[10] Valter Battistoni, Alessandro Montemaggiori and Paolo Iori. "Beyond falconry between tradition and modernity: a new device for bird strike hazard prevention at airports". In: Proceedings of International Bird Strike Committee, IBSC Meeting and Seminario Internacional Perigo Aviario e Fauna. Brasilia. 2008, pp. 1-13.

[11] Julianie Beaulieu, Jaap H. de Wilde and Jacqueline M.A. Scherpen. Smart Grids from a Global Perspective: bridging old and new energy systems. Springer, Feb. 2016. IS BN: 978-3-319-28075-2.

[12] B. Bigland and O. C. J. Lippold. "The relation between force, velocity and integrated electrical activity in human muscles". In: J. Physiol. (123 1954), pp. 214-224.

[13] MIT Biomimetic Robotics Lab. MITcheetah: Cheetah-inspired Quadruped. Feb. 2012. URL: ttp:// sangbae. scripts . mit . edu/biomimetics/ (visited on 08/05/2012).

[14] F. Blaabjerg et al. "Overview of Control and Grid Synchronization for Distributed Power Generation Systems". In: Industrial Electronics, IEEE Transactions on 53.5 (Oct. 2006), pp. 1398-1409. IS SN: 0278-0046. DOI: 10.1109/TIE.2006.881997.

[15] I.I. Blekhman et al. "On self-synchronization and controlled synchronization". In: Systems \& Control Letters 31.5 (1997). Control of Chaos and Synchronization, pp. 299-305. I S N N 0167-6911. DOI: 10.1016/S0167-6911( 97 )00047-9.

[16] BostonDynamics. CHEETAH - fastest legged robot. Mar. 2012. URL: http : / / www . bostondynamics . com/robot_cheetah . html (visited on 08/05/2012).

[17] PC Breedveld. "Modeling and simulation of dynamic systems using bond graphs". In: Encyclopedy of of Life Support Systems (EOLSS) (2008), pp. 1-37.

[18] P.C. Breedveld. "Physical systems theory in terms of bond graphs". $\mathrm{PhD}$ thesis. Technische Hogeschool Twente, Feb. 1984.

[19] Y. Brodskiy. "Robust autonomy for interactive robots". PhD thesis. Enschede: University of Twente, Feb. 2014. DoI: 10 . 3990/1.9789036536202. 
[20] Jonas Buchli, Fumiya Iida and Auke Jan Ijspeert. "Finding resonance: Adaptive frequency oscillators for dynamic legged locomotion". In: In Proceedings iros 2006. (In. Springer Verlag, 2006, pp. 3903-3909.

[21] Matthew Butterick. Practical typography. e-book. Matthew Butterick, 2010. URL: http://pract ical typography . com.

[22] Robbie N. Cada. Black Marlin. Obtained through wikipedia, ht tps: / / en . wikipedia.org / wiki / Black_marlin. 2015. URL: http://www. fishbase.org (visited on 22/02/2017).

[23] M. Kanat Camlibel et al., eds. Mathematical Control Theory I: Nonlinear and Hybrid Control Systems. 1st. Vol. 461. Lecture Notes in Control and Information Sciences. Springer International Publishing, 2015. IS BN: 978-3-319-20987-6. DOI: 10.1007/978-3319-20988-3.

[24] Qu Cao and Ioannis Poulakakis. "Passive Quadrupedal Bounding with a Segmented Flexible Torso". In: Proceedings 2012 IEEE/RSJ International Conference on Intelligent Robots and Systems (IROS2012). 2012, pp. 2484-2489. IS BN: 9781467317351.

[25] PR Cavanagh and MA Lafortune. "Reaction forces in distance running". In: Journal of biomechanics 13 (1980), pp. 397-406.

[26] J. Cervera, A.J. van der Schaft and A. Baños. "Interconnection of port-Hamiltonian systems and composition of Dirac structures". In: Automatica 43.2 (Feb. 2007), pp. 212-225. IS S N: 00051098. DOI: 10.1016/j . automatica.2006.08.014.

[27] P. Chebotarev. "Comments on "Consensus and cooperation in networked multi-agent systems" ". In: Proceedings of the IEEE 98.7 (July 2010), pp. 1353-1354. ISSN: O018-9219. DOI: 10 . 1109/JPROC. 2010.2049911.

[28] Clear Flight Solutions. Robotic bird control. 2016. URL: http : //clearflightsolutions. com (visited on 01/03/2016).

[29] Controllab Products. 20-sim: the power in modeling. Dec. 2016. URL: http://www.20sim.com.

[30] S. Cotton et al. "Fast-Runner: A Fast, Efficient and Robust Bipedal Robot, Concept and Planar Simulation". In: Proc. IEEE International Conference on Robotics and Automation (ICRA). 2012.

[31] John David Crawford. "Amplitude expansions for instabilities in populations of globally-coupled oscillators". In: Journal of Statistical Physics 74.5 (1994), pp. 1047-1084. IS SN: 1572-9613. DOI: $10.1007 / B F 02188217$. 
[32] G.C.H.E. de Croon et al. "Design, aerodynamics, and visionbased control of the DelFly". In: International Journal of Micro Air Vehicles 1.2 (2009), pp. 71-97. IS SN: 1756-8293.

[33] U. Culha and U. Saranli. "Quadrupedal bounding with an actuated spinal joint". In: Robotics and Automation (ICRA), 2011 IEEE International Conference on. IEEE, May 2011, pp. 1392-1397. IS BN: 978-1-61284-386-5. DOI: 10.1109/ICRA.2011.5980176.

[34] Demcon. analyze. create. achieve. 2012. URL: http://demcon. $\mathrm{nl} /$ (visited on 08/05/2012).

[35] Edwin Dertien. "Realisation of an energy-efficient walking robot". M.Sc. thesis. University of Twente, June 2005. URL: ht tp: //purl.utwente.nl/essays/56911.

[36] Desoky and Abd El-Aleem Saad Soliman. "A Review of Bird Control Methods at Airports". In: Global Journal of Science Frontier Research 14.2 (2014).

[37] Douwe Dresscher. "Controlled Passive Actuation: concepts for energy efficient actuation using mechanical storage elements and continuously variable transmissions". $\mathrm{PhD}$ thesis. University of Twente, Robotics and Mechatronics group, 2016. IS BN: 97890-365-4188-6. DOI: 10.3990/1.9789036541886.

[38] Vincent Duindam and Stefano Stramigioli. "Port-Based Asymptotic Curve Tracking for Mechanical Systems". In: European Journal of Control 10.5 (2004), pp. 411-420.

[39] Vincent Duindam et al. Modeling and Control of Complex Physical Systems. Berlin, Heidelberg: Springer Berlin Heidelberg, 2009. IS BN: 978-3-642-03195-3. DOI: 10 . 1007/978-3-642-031960.

[40] Dutch Institute for Systems and Control. DISC field \& themes. 2012. URL: http://www. disc. tudelft.nl/research/discfield-themes. html (visited on 08/05/2012).

[41] Boston Dynamics. Robots. 2014. URL: http://www. bostondynamics. $\mathrm{com} /$ index. html (visited on 01/10/2015).

[42] ED Fasse and PC Breedveld. "Modeling of Elastically Coupled Bodies: Part II-Exponential and Generalized Coordinate Methods". In: Journal of dynamic systems, measurement, and control 120 (1998), pp. 501-506.

[43] James Ferguson-Lees and David A Christie. Raptors of the world. Houghton Mifflin Harcourt, 2001.

[44] Festo. SmartBird - bird flight deciphered. 2015. URL: http : // www . festo. com/cms / en\%5C_corp/11369. htm (visited on 01/04/2015). 
[45] Gerrit A. Folkertsma, Sangbae Kim and Stefano Stramigioli. "Parallel stiffness in a bounding quadruped with flexible spine". In: 2012 IEEE/RSJ International Conference on Intelligent Robots and Systems. Oct. 2012, pp. 2210-2215. DOI: 10 . 1109 / IROS . 2012.6385870.

[46] Gerrit A. Folkertsma, Arjan J. van der Schaft and Stefano Stramigioli. "Morphological computation in a fast-running quadruped with elastic spine". In: Proc. Lagrangian and Hamiltonian Methods for Nonlinear Control (LHMNLC15) (July 2015).

[47] Gerrit A. Folkertsma, Arjan J. van der Schaft and Stefano Stramigioli. "Power-continuous synchronisation of oscillators: A novel, energy-free way to synchronise dynamical systems". In: Robotics and Automation (ICRA), 2014 IEEE International Conference on. May 2014, pp. 1493-1498. DOI: 10.1109/ICRA. 2014.6907049.

[48] M C J Franken et al. "Bridging the gap between passivity and transparency". In: Proceedings of Robotics: Science and Systems. Seattle, USA: Robotics Science and Systems, June 2009, p. 36. DOI: 10.15607/RSS.2009.V.036.

[49] Rudolf M. Füchslin et al. "Morphological Computation and Morphological Control: Steps Toward a Formal Theory and Applications". In: Artificial Life 19.1 (Nov. 2012), pp. 9-34. ISSN: 1064-5462. DOI: 10.1162/ARTL_a_00079.

[50] R J Full and D E Koditschek. "Templates and anchors: neuromechanical hypotheses of legged locomotion on land." In: The Journal of experimental biology 202.Pt 23 (Dec. 1999), pp. 3325-32. ISSN: 0022-0949.

[51] R. M. Ghigliazza et al. "A Simply Stabilized Running Model". In: SIAM Rev. 47.3 (Mar. 2005), pp. 519-549. IS SN: 0036-1445. DOI: $10.1137 / 050626594$.

[52] G. Gilardi and I. Sharf. "Literature survey of contact dynamics modelling". In: Mechanism and Machine Theory 37.10 (Oct. 2002), pp. 1213-1239. IS SN: 0094114X. DOI: 10.1016/S0094$114 \times(02) 00045-9$.

[53] Leon Glass. "Synchronization and rhythmic processes in physiology". In: Nature 410.6825 (Mar. 2001), pp. 277-284. IS SN: 0028-0836. DOI: $10.1038 / 35065745$.

[54] SA Gracovetsky and S Iacono. "Energy transfers in the spinal engine". In: Journal of biomedical engineering 9.2 (1987), pp. 99114 . 
[55] F. Guenther and F. Iida. "Energy-Efficient Monopod Running With a Large Payload Based on Open-Loop Parallel Elastic Actuation". In: IEEE Transactions on Robotics PP.99 (2017), pp. 1-12. IS N N: 1552-3098. DOI: 10.1109/TR0.2016.2623342.

[56] M Haberland and S Kim. "On extracting design principles from biology: I. Method-General answers to high-level design questions for bioinspired robots". In: Bioinspiration \& Biomimetics 10.1 (2015), p. 016010.

[57] Sami Haddadin et al. "It Is (Almost) All about Human Safety: A Novel Paradigm for Robot Design, Control, and Planning". English. In: Computer Safety, Reliability, and Security. Ed. by Friedemann Bitsch, Jérémie Guiochet and Mohamed Kaâniche. Vol. 8153. Lecture Notes in Computer Science. Springer Berlin Heidelberg, 2013, pp. 202-215. IS BN: 978-3-642-40792-5. DOI: 10.1007/978-3-642-40793-2_19.

[58] V.R. Ham et al. "Compliant actuator designs: Review of actuators with passive adjustable compliance/controllable stiffness for robotic applications". In: IEEE Robotics and Automation Magazine 16.3 (2009), pp. 81-94. DOI: 10.1109/MRA. 2009.933629.

[59] F. Hardarson. Locomotion for difficult terrain. Tech. rep. Royal Institute of Technology, Stockholm, Sweden, 1997.

[6o] S.A. Hartman, H.W.M. Hoeijmakers and R.J. Musters. "Experimental investigation of flow about wing of robot bird". In: 5oth AIAA Aerospace Sciences Meeting Including the New Horizons Forum and Aerospace Exposition. 2012.

[61] BM Haueisen. "Investigation of an Articulated Spine in a Quadruped Robotic System". PhD thesis. University of Michigan, 2011.

[62] Helmut Hauser et al. "Towards a theoretical foundation for morphological computation with compliant bodies". In: Biological Cybernetics 105.5 (2011), pp. 355-370. IS SN: 1432-0770. DOI: $10.1007 / \mathrm{s} 00422-012-0471-0$.

[63] Just L. Herder. "Design of spring force compensation systems". In: Mechanism and Machine Theory 33.1 (Jan. 1998), pp. 151-161. DOI: 10.1016/S0094-114X( 97 )00027-X.

[64] Harry W. Hoeijmakers and Joost Mulder. "Computational and Experimental Investigation into Flapping Wing Propulsion". In: 54th AIAA Aerospace Sciences Meeting. American Institute of Aeronautics and Astronautics, Jan. 2016. DOI: doi : 10 . 2514 / 6 . 2016-0802. 
[65] Neville Hogan. "Impedance control: an approach to manipulation". In: Journal of Dynamic Systems, Measurement and Control 107.1 (Mar. 1985), pp. 1-24.

[66] F. N. Hoogeboom, A. Y. Pogromsky and H. Nijmeijer. "Huygens' inspired multi-pendulum setups: Experiments and stability analysis". In: Chaos: An Interdisciplinary Journal of Nonlinear Science 26.11 (2016), p. 116304. DOI: 10.1063/1.4965031.

[67] Penny E. Hudson et al. "Functional anatomy of the cheetah (Acinonyx jubatus) hindlimb". In: Journal of Anatomy 218.4 (2011), pp. 363-374. IS SN: 1469-7580. DOI: $10.1111 / \mathrm{j}$. 1469-7580. 2010.01310.x.

[68] J. Hunt et al. "Improving Efficiency for an Open-Loop-Controlled Locomotion With a Pulsed Actuation". In: IEEE/ASME Transactions on Mechatronics 21.3 (June 2016), pp. 1581-1591. ISSN: 1083-4435. DOI: 10.1109/TMECH. 2016.2539206.

[69] K. H. Hunt and F. R. E. Crossley. "Coefficient of Restitution Interpreted as Damping in Vibroimpact". In: Journal of Applied Mechanics 42.2 (1975), pp. 440-445. DOI: 10.1115/1.3423596.

[70] J.W. Hurst, J.E. Chestnutt and AA Rizzi. "An actuator with physically variable stiffness for highly dynamic legged locomotion". In: Robotics and Automation, 2004. Proceedings. ICRA '04. 2004 IEEE International Conference on. Vol. 5. Apr. 2004, 4662-4667 Vol.5. DOI: 10.1109/ROBOT.2004.1302453.

[71] Sang-Ho Hyon and T. Emura. "Running control of a planar biped robot based on energy-preserving strategy". In: Robotics and Automation, 2004. Proceedings. ICRA '04. 2004 IEEE International Conference on. Vol. 4. May 2004, 3791-3796 Vol.4. DOI: 10.1109/ROBOT.2004. 1308859.

[72] Fumiya Iida, G Gómez and Rolf Pfeifer. "Exploiting body dynamics for controlling a running quadruped robot". In: Advanced Robotics, 2005. ICAR' '05. Proceedings., 12th International Conference on. 2005, pp. 229-235. ISBN: 0780391772. DOI: 10.1109/ ICAR. 2005. 1507417.

[73] Auke Jan Ijspeert et al. "From swimming to walking with a salamander robot driven by a spinal cord model". In: Science 315.5817 (2007), pp. 1416-1420.

[74] Robert D. Jansen. Digital Art: "Phantoms - Bat Cave". 2017. URL: http://www. artpal.com/rdjansen (visited on 09/03/2017).

[75] Theo Jansen. Strandbeest. 2016. URL: http://strandbeest . com (visited on 01/11/2016). 
[76] M.H.H. Kani et al. "Effect of flexible spine on stability of a passive quadruped robot: Experimental results". In: Robotics and Biomimetics (ROBIO), 2011 IEEE International Conference on. Dec. 2011, pp. 2793-2798. DOI: 10.1109/ROBI0.2011.6181728.

[77] Mahdi Khoramshahi et al. "Piecewise linear spine for speed-energy efficiency trade-off in quadruped robots". In: Robotics and Autonomous Systems 61.12 (2013), pp. 1350-1359. ISSN: 09218890. DOI: $10.1016 / \mathrm{j}$. robot .2013 .08 .001 .

[78] Sangbae Kim, Cecilia Laschi and Barry Trimmer. "Soft robotics: a bioinspired evolution in robotics". In: Trends in Biotechnology 31.5 (2013), pp. 287-294. ISSN: 0167-7799. DOI: 10.1016/ j . tibtech.2013.03.002.

[79] Y. Kuramoto. Chemical Oscillations, Waves, and Turbulence. Springer, Berlin, 1984.

[8o] M. Laffranchi, N.G. Tsagarakis and D.G. Caldwell. "Safe human robot interaction via energy regulation control". In: Intelligent Robots and Systems, 2009. IROS 2009. IEEE/RSJ International Conference on. Oct. 2009, pp. 35-41. DOI: 10.1109/IR0S.2009. 5354803.

[81] D. Lakatos, F. Petit and A. Albu-Schaffer. "Nonlinear oscillations for cyclic movements in variable impedance actuated robotic arms". In: Robotics and Automation (ICRA), 2013 IEEE International Conference on. May 2013, pp. 508-515. DOI: 10.1109/ ICRA. 2013.6630622.

[82] Dominic Lakatos et al. "A Modally Adaptive Control for MultiContact Cyclic Motions in Compliantly Actuated Robotic Systems". In: International Conference on Intelligent Robots and Systems. Proceedings of IEEE/RSJ International Conference on Intelligent Robots and Systems. Nov. 2013, pp. 5388-5395. DOI: 10.1109/IROS.2013.6697136.

[83] Dongjun Lee and Ke Huang. "Passive-Set-Position-Modulation Framework for Interactive Robotic Systems". In: Robotics, IEEE Transactions on 26.2 (Apr. 2010), pp. 354-369. IS SN: 1552-3098. DOI: 10.1109/TRO.2010.2041877.

[84] S.M. Lukic et al. "Energy storage systems for automotive applications". In: IEEE Transactions on Industrial Electronics 55.6 (2008). cited By 437, pp. 2258-2267. DOI: 10.1109/TIE. 2008.918390.

[85] N. Maheshwari et al. "Resonance based multi-gaited robot locomotion". In: Intelligent Robots and Systems (IROS), 2012 IEEE/RSJ International Conference on. Oct. 2012, pp. 169-174. DOI: 10 . 1109/IROS.2012.6385618. 
[86] Tad McGeer. "Passive Dynamic Walking". In: The International Journal of Robotics Research 9.2 (1990), pp. 62-82. DOI: 10.1177/ 027836499000900206.

[87] Thomas A. McMahon and George C. Cheng. "The mechanics of running: How does stiffness couple with speed?" In: Journal of Biomechanics 23, Supplement 1 (1990). International Society of Biomechanics, pp. 65-78. IS SN: 0021-9290. DOI: 10.1016/ 0021-9290 ( 90 ) 90042-2.

[88] L. Meier, D. Honegger and M. Pollefeys. "PX4: A Node-Based Multithreaded Open Source Robotics Framework for Deeply Embedded Platforms". In: Robotics and Automation (ICRA), 2015 IEEE International Conference on. May 2015.

[89] Renato E. Mirollo and Steven H. Strogatz. "Synchronization of Pulse-Coupled Biological Oscillators". In: SIAM Journal on Applied Mathematics 50.6 (1990), pp. 1645-1662. DoI: 10.1137/ 0150098.

[9o] Brayden Muller et al. "Design of bio-inspired autonomous aircraft for bird management". In: AIAC16: 16th Australian International Aerospace Congress. Engineers Australia. 2015, p. 370.

[91] Ed Obert. Aerodynamic design of transport aircraft. ISBN13 9781586039707. IOS Press Amsterdam, 2009.

[92] R. Olfati-Saber, J.A. Fax and R.M. Murray. "Consensus and Cooperation in Networked Multi-Agent Systems". In: Proceedings of the IEEE 95.1 (Jan. 2007), pp. 215-233. ISSN: 0018-9219. DOI: 10.1109/JPROC.2006.887293.

[93] G. van Oort and S. Stramigioli. "Velocity control of a 2 D dynamic walking robot". In: Proceedings of the 26th Benelux Meeting on Systems and Control, Lommel, België, edited by Pintelon, R., Schoukens, J, Smets, I, 2007, p. 1.

[94] Romeo Ortega et al. "Putting energy back in control". In: IEEE Control Systems 21.2 (Apr. 2001), pp. 18-33. IS N: 1066-033X. DOI: 10.1109/37.915398.

[95] R. Ortega et al. "Control by Interconnection and Standard PassivityBased Control of Port-Hamiltonian Systems". In: Automatic Control, IEEE Transactions on 53.11 (Dec. 2008), pp. 2527-2542. IS S N: 0018-9286. DOI: 10.1109/TAC.2008.2006930.

[96] R. Ortega et al. "On passivity-based output feedback global stabilization of Euler-Lagrange systems". In: Proceedings of 1994 33rd IEEE Conference on Decision and Control. Vol. 1. Dec. 1994, 381-386 vol.1. DOI: 10.1109/CDC. 1994. 410898. 
[97] Hae-Won Park and Sangbae Kim. "Quadrupedal galloping control for a wide range of speed via vertical impulse scaling". In: Bioinspiration \& biomimetics 10.2 (2015), p. 025003.

[98] H.M. Paynter. Analysis and design of engineering systems. M.I.T. Press, MA, 1960.

[99] L.M. Pecora. "Synchronization conditions and desynchronizing patterns in coupled limit-cycle and chaotic systems". In: Physical Review E - Statistical Physics, Plasmas, Fluids, and Related Interdisciplinary Topics 58.1 (1998), pp. 347-36o.

[100] Louis M. Pecora and Thomas L. Carroll. "Synchronization in chaotic systems". In: Phys. Rev. Lett. 64 (8 Feb. 1990), pp. 821824. DOI: 10.1103/PhysRevLett.64.821.

[101] R. Pfeifer and F. Iida. Embodied Artificial Intelligence. Springer, 2003.

[102] Rolf Pfeifer. Artificial intelligence: time to "invest in soft robotics". Apr. 2012. URL: http : / / wWw . publicserviceeurope.com / article/1772/time-to-wake-up-and-invest-heavilyin-robotics-technology (visited on 08/05/2012).

[103] Rolf Pfeifer and Gabriel Gómez. "Morphological Computation - Connecting Brain, Body, and Environment". English. In: Creating Brain-Like Intelligence. Ed. by Bernhard Sendhoff et al. Vol. 5436. Lecture Notes in Computer Science. Springer Berlin Heidelberg, 2009, pp. 66-83. IS BN: 978-3-642-00615-9. DOI: 10.1007/978-3-642-00616-6_5.

[104] Rolf Pfeifer, Max Lungarella and Fumiya Iida. "Self-Organization, Embodiment, and Biologically Inspired Robotics". In: Science 318.5853 (2007), pp. 1088-1093. DOI: 10.1126/science. 1145803.

[105] Rolf Pfeifer, Stefano Stramigioli et al. Robot Companions for Citizens, FETflagship. 2012. URL: http://www. robotcompanions . eu/project (visited on 08/05/2012).

[106] M.F. Platze et al. "Flapping-wing aerodynamics: Progress and challenges". In: AIAA Journal 46 (2008), pp. 2136-2149.

[107] M. Plooij and M. Wisse. "A novel spring mechanism to reduce energy consumption of robotic arms". In: 2012 IEEE/RSJ International Conference on Intelligent Robots and Systems. Oct. 2012, pp. 2901-2908. DOI: 10.1109/IROS.2012.6385488.

[108] I. Poulakakis. "On the Stability of the Passive Dynamics of Quadrupedal Running with a Bounding Gait". In: The International Journal of Robotics Research 25.7 (July 2006), pp. 669-687. ISSN: 0278-3649. DOI: $10.1177 / 0278364906066768$. 
[109] Soha Pouya et al. "Role of Spine Compliance and Actuation in the Bounding Performance of Quadruped Robots". In: Dynamic Walking Conference. 2012.

[110] G. A. Pratt and M. M. Williamson. "Series elastic actuators". In: Intelligent Robots and Systems 95. 'Human Robot Interaction and Cooperative Robots', Proceedings. 1995 IEEE/RSJ International Conference on. Vol. 1. Aug. 1995, 399-406 vol.1. DOI: 10.1109/ IROS. 1995.525827.

[111] Jason L. Pusey et al. "Free-standing leaping experiments with a power-autonomous elastic-spined quadruped". In: Proc. SPIE. Vol. 8741. 2013, 87410W-87410W-15. DOI: 10.1117/12.2016073.

[112] Marc Raibert. Legged robots that balance. MIT Press, 1986.

[113] Marc H. Raibert. "Trotting, pacing and bounding by a quadruped robot". In: Journal of Biomechanics 23, Supplement 1 (1990). $<$ ce:title>International Society of Biomechanics</ce:title>, pp. 7998. IS SN: 0021-9290. DOI: 10. 1016/0021-9290( 90 ) 90043-3.

[114] RAM. Robotics and Mechatronics. 2012. URL: https://www.ce. utwente.nl/ (visited on 08/05/2012).

[115] A. Rodriguez-Angeles and H. Nijmeijer. "Mutual synchronization of robots via estimated state feedback: a cooperative approach". In: IEEE Transactions on Control Systems Technology 12.4 (July 2004), pp. 542-554. ISSN: 1063-6536. DOI: 10.1109/ TCST. 2004.825065.

[116] Michael G. Rosenblum, Arkady S. Pikovsky and Jürgen Kurths. "Phase Synchronization of Chaotic Oscillators". In: Phys. Rev. Lett. 76 (11 Mar. 1996), pp. 1804-1807. DOI: 10 . $1103 /$ PhysRevLett.76.1804.

[117] Andy Ruina, John E.A. Bertram and Manoj Srinivasan. "A collisional model of the energetic cost of support work qualitatively explains leg sequencing in walking and galloping, pseudoelastic leg behavior in running and the walk-to-run transition". In: Journal of Theoretical Biology 237.2 (2005), pp. 170-192. ISSN: 0022-5193. DOI: 10.1016/j.jtbi.2005.04.004.

[118] Jee-Hwan Ryu, Dong-Soo Kwon and B. Hannaford. "Stable teleoperation with time-domain passivity control". In: Robotics and Automation, IEEE Transactions on 20.2 (Apr. 2004), pp. 365-373. IS SN: 1042-296X. DOI: 10.1109/TRA.2004.824689.

[119] A. Sanchez-Squella et al. "Dynamic Energy Router". In: IEEE Control Systems 30.6 (Dec. 2010), pp. 72-80. IS SN: 1066-033X. DOI: 10.1109/MCS.2010.938096. 
[120] SCAA. Systems, Control and Applied Analysis. 2012. URL: http: //www.math.rug.nl/scaa/ (visited on 08/05/2012).

[121] Luca Scardovi and Rodolphe Sepulchre. "Synchronization in networks of identical linear systems". In: Automatica 45.11 (2009), pp. 2557-2562. IS N N: 0005-1098. DOI: 10.1016/j . automat ica. 2009.07.006.

[122] Arjan van der Schaft. L2-gain and passivity techniques in nonlinear control. 3rd ed. Communications and Control Engineering. Springer International Publishing, 2017. IS B N: 978-3-319-49991-8. DOI: 10.1007/978-3-319-49992-5.

[123] Arjan van der Schaft and Dimitri Jeltsema. "Port-Hamiltonian Systems Theory: An Introductory Overview". In: Foundations and Trends ${ }^{\circledR}$ in Systems and Control 1.2-3 (2014), pp. 173-378. IS SN: 2325-6818. DOI: 10.1561/2600000002.

[124] Jacquelien M.A. Scherpen. "Distributed supply-demand balancing and the physics of smart energy systems". In: European Journal of Control 24.7 (July 2015), pp. 63-71. IS SN: 0947-3580. DOI: 10.1016/j.ejcon.2015.04.005.

[125] C. Schindlbeck and S. Haddadin. "Unified passivity-based Cartesian force/impedance control for rigid and flexible joint robots via task-energy tanks". In: Robotics and Automation (ICRA), 2015 IEEE International Conference on. May 2015, pp. 440-447. DOI: 10.1109/ICRA. 2015.7139036.

[126] William John Schwind. "Spring loaded inverted pendulum running: A plant model". PhD thesis. Ann Arbor, MI, USA: University of Michigan, 1998. IS BN: 0-599-08461-8.

[127] C. Secchi, S. Stramigioli and C. Fantuzzi. "Dealing with unreliabilities in digital passive geometric telemanipulation". In: Proceedings 2003 IEEE/RSJ International Conference on Intelligent

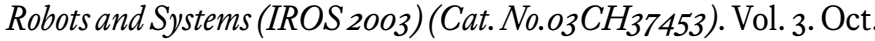
2003, 2823-2828 vol.3. DOI: 10.1109/IROS.2003. 1249298.

[128] A. Seeni et al. "Robot Mobility Concepts for Extraterrestrial Surface Exploration". In: Aerospace Conference, 2008 IEEE. Mar. 2008, pp. 10-14. DOI: 10.1109/AER0.2008.4526237.

[129] C Semini et al. "Design of HyQ - a hydraulically and electrically actuated quadruped robot". In: Proceedings of the Institution of Mechanical Engineers, Part I: Journal of Systems and Control Engineering (2011). DOI: 10.1177/0959651811402275. 
[130] S. Seok et al. "Design Principles for Energy-Efficient Legged Locomotion and Implementation on the MIT Cheetah Robot". In: IEEE/ASME Transactions on Mechatronics 20.3 (June 2015), pp. 1117-1129. IS SN: 1083-4435. DOI: 10. 1109/TMECH. 2014. 2339013.

[131] Richard Shepherd Shevell. Fundamentals offlight. 2nd ed. ISBN13 978-0133390605. Prentice Hall Englewood Cliffs, New Jersey, 1989.

[132] A.S. Shiriaev and A.L. Fradkov. "Stabilization of invariant sets for nonlinear non-affine systems". In: Automatica 36.11 (2000), pp. 1709-1715. DOI: 10.1016/S0005-1098(00)00077-7.

[133] A.S. Shiriaev and A.L. Fradkov. "Stabilization of invariant sets for nonlinear systems with applications to control of oscillations". In: International Journal of Robust and Nonlinear Control 11.3 (2001), pp. 215-240. DOI: 10.1002/rnc. 568.

[134] A.S. Shiriaev, L.B. Freidovich and S.V. Gusev. "Transverse Linearization for Controlled Mechanical Systems With Several Passive Degrees of Freedom". In: Automatic Control, IEEE Transactions on 55.4 (Apr. 2010), pp. 893-906. IS N: 0018-9286. DOI: 10.1109/TAC.2010.2042000.

[135] Christopher Simpkins. Hack: a typeface designed for source code. 2015. URL: http://sourcefoundry.org/hack/.

[136] John Simpson and Edmund Weiner, eds. The Oxford English Dictionary. Oxford University Press, 1989. IS BN: 978-0-19-861186-8.

[137] Meera Sitharam and Menghan Wang. "How the Beast really moves: Cayley analysis of mechanism realization spaces using CayMos". In: Computer-Aided Design 46 (2014). 2013 SIAM Conference on Geometric and Physical Modeling, pp. 205-210. IS SN: 0010-4485. DOI: 10.1016/j.cad.2013.08.033.

[138] A.C. Smith and M.D. Berkemeier. "Passive dynamic quadrupedal walking". In: Robotics and Automation, 1997. Proceedings., 1997 IEEE International Conference on. Vol. 1. Apr. 1997, 34-39 vol.1. DOI: 10.1109/ROBOT. 1997.620012.

[139] Stefano Stramigioli. "Creating Artificial Damping By Means Of Damping Injection". In: Proceedings of the ASME Dynamic Systems and Control Division. Ed. by K.Danai. Vol. DSC.58. Atlanta, (GE), 1996, pp. 601-606. 
[140] Stefano Stramigioli. "Energy-Aware Robotics". English. In: Mathematical Control Theory I: Nonlinear and Hybrid Control Systems. Ed. by M. Kanat Camlibel et al. Vol. 461. Lecture Notes in Control and Information Sciences. Springer International Publishing, 2015, pp. 37-50. IS BN: 978-3-319-20987-6. DOI: 10.1007/9783-319-20988-3.

[141] Stefano Stramigioli. Modeling and IPC control of interactive mechanical systems: a coordinate-free approach. Vol. 266. Springer, 2001.

[142] Stefano Stramigioli and M. Dijk. "Energy Conservative Limit Cycle Oscillations". In: 17th IFAC World Congress. 2008, pp. 1566615671. IS BN: 9781123478.

[143] Stefano Stramigioli, Ernie Fasse and Jan C. Willems. "A rigorous framework for interactive robot control". In: International Journal of Control 75.18 (2002), pp. 1486-1502. DOI: 10.1080/ 0020717021000032078.

[144] Stefano Stramigioli, Bernhard Maschke and Arjan J van der Schaft. "Passive output feedback and port interconnection". In: In Proceedings of 4th IFAC NOLCOS. Enschede, Netherlands, 1998, pp. 613-618.

[145] S. Stramigioli et al. "A novel theory for sampled data system passivity". In: IEEE/RSJ International Conference on Intelligent Robots and System, 2002. Vol. 2. IEEE, 2002, pp. 1936-1941.

[146] S. Stramigioli et al. "Sampled Data Systems Passivity and Discrete Port-Hamiltonian Systems". In: Robotics, IEEE Transactions on 21.4 (Aug. 2005), pp. 574-587. IS SN: 1552-3098. DOI: $10.1109 /$ TRO 2004.842330 .

[147] S.H. Strogatz. "From Kuramoto to Crawford: Exploring the onset of synchronization in populations of coupled oscillators". In: Physica D: Nonlinear Phenomena 143.1-4 (2000), pp. 1-20.

[148] J.A.K. Suykens, P.F. Curran and L.O. Chua. "Robust synthesis for master-slave synchronization of Lur'e systems". In: IEEE Transactions on Circuits and Systems I: Fundamental Theory and Applications 46.7 (1999), pp. 841-850. DOI: 10.1109/81.774230.

[149] T.S. Tadele, T. de Vries and S. Stramigioli. "The Safety of Domestic Robotics: A Survey of Various Safety-Related Publications". In: Robotics Automation Magazine, IEEE 21.3 (Sept. 2014), pp. 134-142. IS S N: 1070-9932. DOI: 10.1109/MRA. 2014. 2310151. 
[150] S. Talebi et al. "Quadruped Robot Running with a Bounding Gait". In: Experimental Robotics VII. Vol. 271. Lecture Notes in Control and Information Sciences. Springer Berlin / Heidelberg, 2001, pp. 281-290.

[151] G.K. Taylor, R.L. Nudds and A.L.R. Thomas. "Flying and swimming animals cruise at a Strouhal number tuned for high power efficiency". In: Nature 425 (2003), pp. 707-711.

[152] Jun-nosuke Teramae and Dan Tanaka. "Robustness of the NoiseInduced Phase Synchronization in a General Class of Limit Cycle Oscillators". In: Phys. Rev. Lett. 93 (20 Nov. 2004), p. 204103. DOI: 10.1103/PhysRevLett.93.204103.

[153] N.G. Tsagarakis et al. "A compact soft actuator unit for small scale human friendly robots". In: Robotics and Automation, 2009. ICRA 'og. IEEE International Conference on. May 2009, pp. 43564362. DOI: 10.1109/ROBOT. 2009.5152496.

[154] V. A. Tucker. "The Energetic Cost of Moving About: Walking and running are extremely inefficient forms of locomotion. Much greater efficiency is achieved by birds, fish-and bicyclists". In American Scientist 63.4 (1975), pp. 413-419. IS S N: 00030996. DOI: $10.2307 / 27845576$.

[155] M. Uemura and S. Kawamura. "Resonance-based motion control method for multi-joint robot through combining stiffness adaptation and iterative learning control". In: Robotics and Automation, 2009. ICRA 'og. IEEE International Conference on. May 2009, pp. 1543-1548. DOI: 10.1109/ROBOT.2009.5152717.

[156] B. Vanderborght et al. "Comparison of mechanical design and energy consumption of adaptable, passive-compliant actuators". In: International Journal of Robotics Research 28.1 (2009), pp. 90103. DOI: $10.1177 / 0278364908095333$.

[157] B. Vanderborght et al. "Variable impedance actuators: A review". In: Robotics and Autonomous Systems 61.12 (2013), pp. 1601-1614. DOI: $10.1016 / j$. robot .2013 .06 .009$.

[158] Leonardo da Vinci. Codice sul volo degli uccelli. Biblioteca Reale di Torino, 1505.

[159] L. C. Visser, R. Carloni and S. Stramigioli. "Energy-Efficient Variable Stiffness Actuators". In: IEEE Transactions on Robotics 27.5 (Oct. 2011), pp. 865-875. ISSN: 1552-3098. DOI: 10.1109/ TR0.2011.2150430. 
[160] Ludo C. Visser, Stefano Stramigioli and Antonio Bicchi. "Embodying Desired Behavior in Variable Stiffness Actuators". In: \{IFAC\} Proceedings Volumes 44.1 (2011). 18th \{IFAC $\}$ World Congress, pp. 9733-9738. IS SN: 1474-6670. DOI: 10.3182/201108286-IT-1002.02202.

[161] Richard Volpe and Pradeep Khosla. "The equivalence of secondorder impedance control and proportional gain explicit force control". In: The International journal of robotics research 14.6 (1995), pp. 574-589.

[162] H.Q. Vu et al. "A variable stiffness mechanism for improving energy efficiency of a planar single-legged hopping robot". In: Advanced Robotics (ICAR), 2013 16th International Conference on. Nov. 2013, pp. 1-7. DOI: 10.1109/ICAR. 2013.6766488.

[163] I. Wanders, G. A. Folkertsma and S. Stramigioli. "Design and analysis of an optimal hopper for use in resonance-based locomotion". In: 2015 IEEE International Conference on Robotics and Automation (ICRA). May 2015, pp. 5197-5202. DOI: 10.1109/ ICRA. 2015.7139923.

[164] Jan C Willems. "Dissipative dynamical systems part I: General theory". In: Archive for rational mechanics and analysis 45.5 (1972), pp. 321-351.

[165] Gregory Wilson. Cheetah's on the Edge-Director's Cut. 2012. URL: https://vimeo.com/53914149 (visited on 30/10/2015).

[166] S. Wolf et al. "Variable Stiffness Actuators: Review on Design and Components". In: IEEE/ASME Transactions on Mechatronics 21.5 (Oct. 2016), pp. 2418-2430. ISSN: 1083-4435. DOI: 10. 1109/TMECH. 2015.2501019.

[167] Justin Won, Stefano Stramigioli and Neville Hogan. "Comment on "The Equivalence of Second-Order Impedance Control and Proportional Gain Explicit Force Control" ". In: The International Journal of Robotics Research 16.6 (1997), pp. 873-875. DOI: 10. 1177/027836499701600610.

[168] Robert J Wood, John P Whitney and Benjamin M Finio. "Mechanics and Actuation for Flapping-Wing Robotic Insects". In: $E n$ cyclopedia of aerospace engineering (2010).

[169] Xiaoxiang Yu and F. Iida. "Minimalistic Models of an EnergyEfficient Vertical-Hopping Robot". In: Industrial Electronics, IEEE Transactions on 61.2 (Feb. 2014), pp. 1053-1062. IS SN: 0278-0046. DOI: 10.1109/TIE.2013.2266080. 
[170] Qian Zhao et al. "Embodiment enables the spinal engine in quadruped robot locomotion". In: Intelligent Robots and Systems (IROS), 2012 IEEE/RSJ International Conference on. Oct. 2012, pp. 2449-2456. DOI: 10.1109/IROS.2012.6386048.

[171] Q. Zhao et al. "Spine dynamics as a computational resource in spine-driven quadruped locomotion". In: 2013 IEEE/RSJ International Conference on Intelligent Robots and Systems. Nov. 2013, pp. 1445-1451. DOI: 10.1109/IROS.2013.6696539. 
Appendix A

\section{Original research proposal}

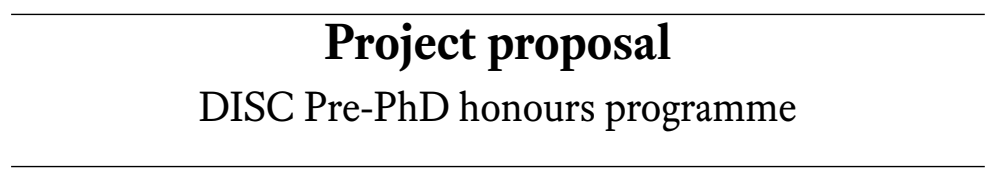

\section{FREEQ}

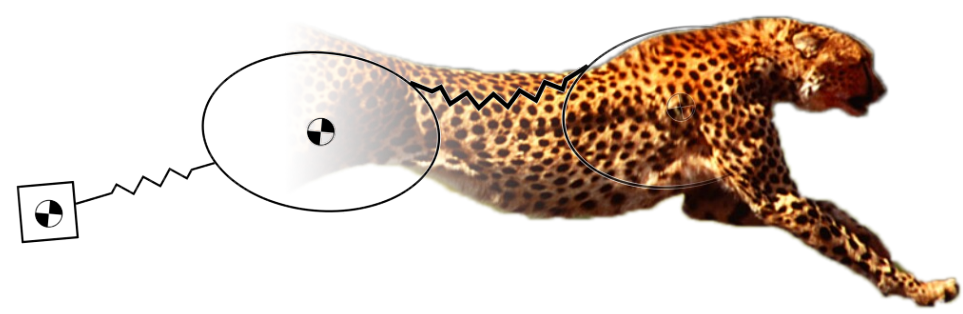

Fast-Running Energy-Efficient Quadruped

Geert Folkertsma

May 2012 


\section{§1 Proposal details}

\begin{tabular}{|c|c|}
\hline Title & Fast-Running Energy-Efficient Quadruped (FREEQ) \\
\hline Groups & $\begin{array}{l}\text { Robotics and Mechatronics, University of Twente } \\
\text { Systems, Control and Applied Analysis, University of } \\
\text { Groningen }\end{array}$ \\
\hline Summary & $\begin{array}{l}\text { The development of legged robots has come a long way, } \\
\text { but concerning speed and energy efficiency there are } \\
\text { still crucial steps to be taken. For inspiration we can } \\
\text { take a look at nature, where quadrupedal runners far } \\
\text { outperform anything mankind has produced thus far. } \\
\text { Attempts to make high-performance quadrupeds are of- } \\
\text { ten based on a mechanical design that "looks like" an } \\
\text { animal such as the leopard. From there, controllers are } \\
\text { optimised for speed, energy or stability-but this will } \\
\text { not yield the desired performance. } \\
\text { Embodied (artificial) intelligence or morphological com- } \\
\text { putation is the idea that system behaviour is the result } \\
\text { of the geometrical interconnection of elements such as } \\
\text { springs and inertias. This embodied AI leads to inher- } \\
\text { ent behaviour, in this project a (fast, efficient) running } \\
\text { motion. } \\
\text { Port-Hamiltonian Systems (PHS) theory models phys- } \\
\text { ical systems as the (geometrical) interconnection of } \\
\text { energy storage and dissipation elements, which not } \\
\text { only gives direct insight to the power flows, but makes } \\
\text { PHS theory by its nature the perfect candidate to study } \\
\text { morphological computation. } \\
\text { In this research project we will design a quadrupedal } \\
\text { runner that has inherent running behaviour, caused } \\
\text { by proper interconnection of storage elements: then a } \\
\text { control system only needs to supply energy to keep this } \\
\text { motion going and stabilise it when necessary, resulting } \\
\text { in a fast, energy-efficient running quadruped. } \\
\text { As inspiration for the design we will look at nature's } \\
\text { best runners-not by copying the mechanical design, } \\
\text { but by identifying them as a PHS and mimic the ener- } \\
\text { getic behaviour, copying that embodied AI that leads } \\
\text { to running motions. }\end{array}$ \\
\hline Supervisors & $\begin{array}{l}\text { prof. dr. ir. S. Stramigioli, RAM, UT } \\
\text { prof. dr. A.J. van der Schaft, SCAA, RuG }\end{array}$ \\
\hline
\end{tabular}




\section{$\S 2$ Description of proposed research}

A large part of the earth is inaccessible to wheeled or tracked vehicles. Were it only the rainforests of Brazil and the peaks of the Himalayas this might be considered a moot case, but our everyday life is filled with stairs, berms, dikes - an unreachable subspace for wheeled robots. If we want robots to perform tasks for us or to cooperate with us in our own environment, they need to manage that environment: they have to move on legs $[59,128]$.

For a stable stance and a large static support base during tasks (e.g. manipulation, inspection), a quadruped is indispensable. A high-performance quadruped, with respect to speed and energy-efficiency, can form the basis for a robot that can function in our environment.

Energy-efficient, fast quadrupedal locomotion is an enabling technology for future robotics.

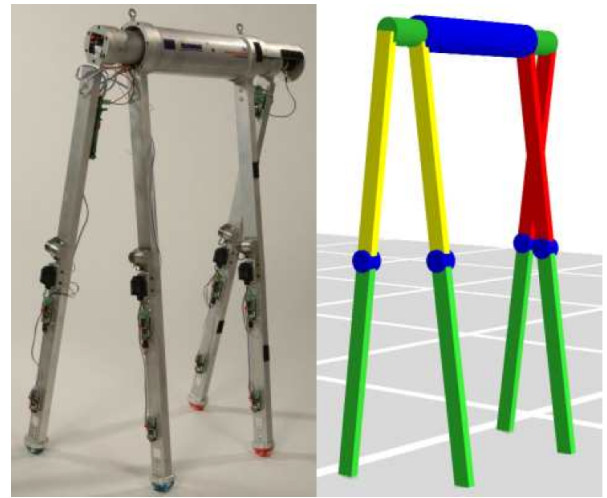

(a) Dribble, a McGeer-inspired walker [93].

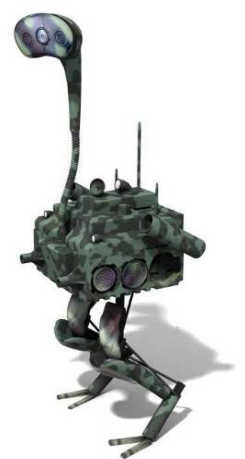

(b) FastRunner, an energy-efficient runner [30].

Figure A.1 - Bipedal, energy-efficient robots.

\section{§2.1 Energy-efficient locomotion}

Energy-efficiency can be attempted to achieve by optimising a controller for minimal power consumption on a given robotic system, but this will generally not yield good results. The real mechatronic approach is to optimise the design of hardware and software, mechanics and electronics, together. The passive-dynamic walkers by McGeer show that walking bipeds can be very efficient, requiring only a little energy input to compensate for friction and impact losses [86] (Figure Figure A.1a). Similar 
designs have not succeeded for quadrupeds, however [138].

When higher speeds are required, the stiff McGeer design does not suffice anymore: the high velocities require compliance in the design to minimise impact losses. For the bipedal runner, a low mechanical cost-oftransport has been realised using the Spring-Loaded Inverted Pendulum (SLIP) model, but because these still use electric or hydraulic actuators without much use of passive elements, practical power consumption is still high $[126,71,112]$. One of the first bipedal runners that actually has a very low electrical cost-of-transport due to the use of passive storage elements is the FastRunner ostrich-inspired robot that is now being developed at IHMC (Jerry Pratt) and the MIT Leg laboratory (Russ Tedrake) [3o] (Figure Figure A.1b).

\section{$\S 2.2$ Fast energy-efficient quadrupedal running robot}

So far, no energy-efficient fast-running quadrupedal robot has been developed yet. The best-known dynamic runner, BostonDynamic's BigDog, has a 15 -hp $(11.2 \mathrm{~kW})$ combustion engine on its back; its follow-up is not much more efficient $[16,13]$. The Scout-II robot [150] and one of Raibert's quadrupeds [113] perform better-energy-wise-but there are none that come close to fast passive-dynamic running. This project, FREEQ, will therefore focus on the development of a quadrupedal, energy-efficient fast-running robot.

Morphological computation The McGeer-type walkers mentioned above, as well as the FastRunner robot, all have certain behaviour built into the mechanical system: their passive behaviour is already close to the desired motions (walking, running), which means that very little control action is required to keep that motion going. This morphological computation $[105,102]$ is also an important aspect of the FREEQproject.

Port-Hamiltonian formulation Morphological computation is possible through the physical interconnection of storage elements, i.e. (multidimensional) springs and inertias. Port-Hamiltonian Systems (PHS) theory provides a framework for modelling physical systems in precisely this manner: the interconnection (called "Dirac structure" in PHS) is a connection of power ports and thus directly shows the topology of the energy flows. The use of PHS for control is also known as Control by Interconnection [95].

Biomimetics It is no coincidence that the FastRunner robot is inspired by an ostrich: through evolution, nature has provided us with optimised designs for various tasks. By studying quadrupedal runners in nature, we can learn how to build them ourselves. In this project it doesn't make 
much sense to try and copy for example a leopard exactly, but rather to extract useful knowledge that can be applied. We want to identify the animal's motions within the PHS framework: what elements are present; can we identify the Dirac structure? Once this step is taken, we can analyse recordings of a running leopard to extract parameters to be used in the FREEQrobot. In subsection A.2.3, this strategy is explained in an example.

Validation The research project presented in this proposal is rather fundamental, in the sense that there is no direct application and a large part of it is trying to understand the principles involved in high-performance quadrupedal motion. Apart from applying these principles to a robot model in simulation, it would be great to do a validation on an actual robot. Within this DISC program, there is unfortunately almost no funding for this. However, the RAM group has good contacts with various mechatronic companies in the region, which have worked together with RAM in the past. If this project gets funded by DISC, collaboration with companies will be sought to build a robot setup to validate the theoretical results.

One of the companies in the LEO center for service robotics that has already expressed its interest in the project is Demcon, a company "dedicated to the development and production of mechatronic applications" [34].

\section{Novelty of the proposed research}

The field of morphological computation or "embodied artificial intelligence" has not yet been extended to high-speed locomotion [101]. The Port-Hamiltonian Systems theory as a framework to study the effect of morphology or physical interconnection can facilitate this extension. On the one hand, it allows to study what elements should be interconnected in what way; on the other, it may lead to the formulation of new required components such as variable stiffness actuators.

Biomimetic (running) robots have been developed before, but there the biological inspiration usually consists exclusively of mechanisms, i.e. the shape of a robot. In this project, the use of PHS theory enables to study the energetic behaviour instead, looking not only at mechanisms but also at storage elements and their interconnection. This type of biological inspiration is much closer to what we want to achieve: similar fast, energy-efficient locomotion.

Finally, to the best knowledge of the author, there exist no quadrupedal energy-efficient fast-running robots yet. 


\section{$\S 2.3$ Biomimetics (example)}

For efficient quadrupedal locomotion we can learn from nature: presumably, evolution and training have led to efficient morphology and gaits. Granted, muscles and tendons are different from electric motors and springs, but energetically they are alike, e.g. when looking at impedance, or the fact that zero mechanical work but nonzero force does consume (metabolic or electric) energy [12].

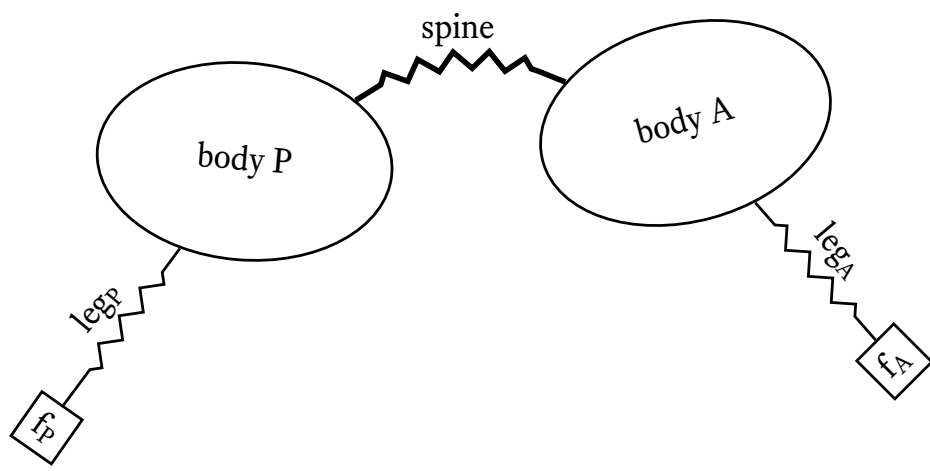

Figure A.2 - Example (planar) quadruped system: anterior $(A)$ and posterior $(P)$ body, feet, and 3-D springs as interconnection.

Suppose a quadrupedal runner, a leopard for instance, could be modelled as in Figure A.2: with spring-like legs, a front or anterior (A) and rear or posterior $(\mathrm{P})$ body and feet, with an elastic spine. The positions of bodies $\mathrm{A}$ and $\mathrm{P}$ are extracted from a video, i.e. $H_{A}(t)$ and $H_{P}(t)$ are known. Then, with inverse dynamics, the 6-D wrenches on these bodies can be found. The wrench on either body is the sum of the forces exerted by the foot and those exerted by the spine (A.1). This way, a biomimetic spinal spring stiffness can be found, which can in turn be applied to a robot design.

$$
\begin{aligned}
& H_{A}(t) \\
& H_{P}(t)
\end{aligned} \Rightarrow \text { inverse dynamics } \Rightarrow \begin{aligned}
& W_{A}(t)=W_{\operatorname{leg}_{A}}(t)+W_{\text {spine }}(t) \\
& W_{B}(t)=W_{\operatorname{leg}_{P}}(t)-W_{\text {spine }}(t)
\end{aligned}
$$




\section{§3 Organisation}

\section{$\S 3.1$ DISC}

This research project will be carried out in a collaboration between two groups within the Dutch Institute for Systems and Control (DISC). The supervisors for this project are professor Stramigioli, of the Robotics and Mechatronics (RAM) group at the University of Twente; and professor Van der Schaft, of the Systems, Control and Applied Analysis (SCAA) group at the University of Groningen. Together, these two groups form a perfect combination to facilitate the research in this project (see below, subsection A.3.2).

Within DISC there are three main research areas, with themes in all areas being addressed in this project [40]:

- System and control theory:

System theory, including behavioural systems, nonlinear, distributed and hybrid systems. Control theory for nonlinear, robust, adaptive, optimal control.

- Theory and application of system modelling:

System identification, estimation and signal processing; Modelling tools: discrete events, hybrid systems, fuzzy logic/neural networks;

- Applications of control engineering:

Mechatronics, robotics, precision technology, motion control systems; Process control and optimisation.

\section{$\S 3.2$ Research groups}

RAM “The science and engineering topics we [Robotics and Mechatronics] work on are: modeling and simulation of physical systems; intelligent control; robotic actuators; embedded control systems." [114]

More specifically, research carried out at the group that is relevant for this project includes design and application of Variable Stiffnes Actuators, bipedal dynamic walkers; and in general the modelling, control and simulation in terms of Port-Hamiltonian Systems, using bond graphs for representation and a simulation package that directly supports this formulation [29].

SCAA "The research program Systems, Control and Applied Analysis is devoted to the analysis and design of complex and heterogeneous system and optimization. The mathematical research in this program is motivated by applications in various areas, including physical engineering 
systems, networked systems, and systems biology." [120]

One of the four main lines of research in this group, the one that is chaired by prof. Van der Schaft, is described as: "Modeling and control of complex physical systems as port-Hamiltonian systems," which of course directly applies to the research in this project.

\section{§3.3 Timetable}

\begin{tabular}{r|r}
\hline Year 1 & DISC courses ${ }^{\dagger}$, Start project, Biomimetic analysis (focus) \\
Year 2 & DISC courses continued ${ }^{\dagger}$, Quadruped system design (focus) \\
Year 3 & Validation (setup, simulation), Optimisation of design \\
Year 4 & Conclusion of research, Thesis writing \\
\hline
\end{tabular}

$\dagger$ Unfortunately, the schedule or availability of DISC courses for 2012-2013 is not available yet, so it is not yet possible to include those more explicitly in the timetable. However, relevant courses that were taught in 2011-2012 and will hopefully be available again, include "Flexible Multibody System Analysis for Control Purposes", "Mathematical Models of Systems", "Nonlinear Control Systems" and "Design Methods for Control Systems".

\section{$\S 3.4$ Budget}

There is a total budget of $€ 10,000$ available for e.g. equipment, consumables, travel (including conference visits).

\begin{tabular}{l|l}
\hline Conferences (registration and accommodation) & $€ 4,000$ \\
Travel & $€ 4,000$ \\
Small equipment, supplies & $€ 2,000$ \\
\hline
\end{tabular}




\section{Appendix B}

\section{Energy control: proof}

The mass-spring system with force actuator, described in (2.39), is interconnected with the passive controller of the form (2.40). If $g$ is chosen as derived in (2.41)-(2.42), the system is exponentially stable at desired energy level $E_{\mathrm{s}}=\frac{k}{2} x_{\mathrm{s}}^{2}$.

Proof. The closed-loop dynamics of the interconnected system are the following:

$$
\begin{aligned}
\mathcal{H}_{\text {tot }}(x) & =\frac{p^{2}}{2 \mathrm{~m}}+\frac{\mathrm{k}}{2} x_{\mathrm{m}}^{2}+\frac{q^{2}}{2 \mathrm{C}} \\
\left(\begin{array}{c}
\dot{p} \\
\dot{x}_{\mathrm{m}} \\
\dot{q}
\end{array}\right) & =\left[\begin{array}{ccc}
0 & -1 & g \\
1 & 0 & 0 \\
-g & 0 & 0
\end{array}\right]\left(\begin{array}{c}
p / m \\
\mathrm{k} x_{\mathrm{m}} \\
q / \mathrm{C}
\end{array}\right),
\end{aligned}
$$

with $g$ chosen as

$$
g:=-\frac{\lambda}{2} \frac{\mathrm{C}}{q v}\left(E-E_{\mathrm{s}}\right) ; \quad E:=\frac{p^{2}}{2 \mathrm{~m}}+\frac{\mathrm{k}}{2} x_{\mathrm{m}}^{2} .
$$

Note that there are two issues with this proposed control law, namely: $v=0$ or $q=0$. The latter case corresponds to an empty energy buffer in the controller, which must be avoided-if more energy needs to be injected to reach $E_{\mathrm{s}}$ than is available, it will of course never reach $E_{\mathrm{s}}$. On the contrary, the first case happens often during the oscillations. In practice, $g$ should be set to some constant, e.g. 0 or 1, whenever $v=0$ (or $v<\epsilon$, some small threshold).

Energy-like functions are often chosen as Lyapunov candidate functions. In this case, we can use the actual energy error $E-E_{\mathrm{s}}$ :

$$
V:=\frac{1}{2}\left(E-E_{\mathrm{s}}\right)^{2},
$$


which is radially unbounded and has a single minimum 0 at $E=E_{\mathrm{s}}$. The time derivative of $V$ is derived in (B.4)-(B.9) below. To obtain (B.6), the system dynamics of (B.1) are used; in (B.7) the control law was used and $p / \mathrm{m}$ substituted for $v$, again from the system dynamics.

$$
\begin{aligned}
\frac{\mathrm{d} V}{\mathrm{~d} t} & =\left(E-E_{\mathrm{s}}\right)\left(\frac{\mathrm{d}}{\mathrm{d} t} E\right) \\
& =\left(E-E_{\mathrm{s}}\right)\left(\frac{p}{\mathrm{~m}} \dot{p}+\mathrm{k} x_{\mathrm{m}} \dot{x}_{\mathrm{m}}\right) \\
& =\left(E-E_{\mathrm{s}}\right)\left(\frac{p}{\mathrm{~m}}\left(-\mathrm{k} x_{\mathrm{m}}+g q / \mathrm{C}\right)+\mathrm{k} x_{\mathrm{m}}(p / \mathrm{m})\right) \\
& =\left(E-E_{\mathrm{s}}\right)\left(\frac{p q}{\mathrm{mC}}\left(-\frac{\lambda}{2} \frac{\mathrm{C}}{q v}\left(E-E_{\mathrm{s}}\right)\right)\right) \\
& =\left(E-E_{\mathrm{s}}\right)\left(-\frac{\lambda}{2}\left(E-E_{\mathrm{s}}\right)\right) \\
& =-\lambda V
\end{aligned}
$$

This derivation shows that $\dot{V}$ is strictly negative when $V \neq 0$, except when $p=0$ : then $g$ is set to some constant and (B.6) will be 0 . From LaSalle's invariance principle, the final trajectories of the system will all lie in the set $\{x(t) \mid \dot{V}(x)=0\}$, which is the union of $\{x(t) \mid V(x)=0\}$ and $\{x(t) \mid p=0\}$. The first set is the trajectory where $E=E_{\mathrm{s}}$; the second set is only an invariant set when $p=0 \wedge \dot{p}=0$, i.e. it contains only the trivial trajectory $x_{\mathrm{m}}(t)=0$.

It can be concluded that $V$ decreases exponentially fast with $\lambda$ and hence the system is globally exponentially stable at $E=E_{\mathrm{s}}$, if there is sufficient energy in the control buffer such that $q>0 \forall t$. 


\section{Embedded, Energy-Aware Actuators}

Safety is essential for robots in unknown environments, especially when there is physical Human-Robot Interaction. Control over energy, or passivity, is an effective safety mechanism [125]. However, when the control algorithm is implemented in a discrete-time computer, possibly even in a distributed architecture, computation and communication delays readily lead to loss of passivity and to instability. In this paper we present a way to make the actuators themselves aware of the energy that they inject into the system. Now passivity and stability are always guaranteed, even in situations of total communication loss. These Embedded Energy-Aware Actuators are a model-free passivity and safety layer that make complex robotic systems again dependable, well-behaved and safe. In its relative simplicity, the presented methodology can have deep implications in robotics for safety, tele-manipulation, architectures and the way we develop systems. It is the only open, interconnectable method that ensures passive behaviour in distributed architectures under any kind of time delays or even communication failures.

This paper has been submitted to a number of robotics conferences and the Robotics \& Automation Letters, but it has not yet been accepted.

The main issues are:

- Passivity, required for guaranteed stability, may lead to loss in performance when classical control laws are used. This is quite a paradigm shift in robotics research.

- An important question is not answered in this paper: how to determine the energy budgets?

As indicated in the conclusion, we are working on addressing both points. The manuscript is included in this thesis to document and make it available, regardless of publication status. 


\section{$\S 1$ Introduction}

Robots have left the controlled environment of factories and are slowly coming into our lives. Apart from all cognitive challenges associated with this change, one of the major concerns is safety-especially when there is physical human-robot interaction (pHRI). There are a number of criteria defining "safety" in pHRI: amongst others the Head Injury Criterion, Maximum Power Index, and Maximum Mean Strain Injury Criterion. In general, to meet these criteria - and thus to be safe-the amount of power/energy/force/acceleration transferred to the human must be limited [5].

The best way to improve a robot's safety is to move away from stiff, heavy, rigidly-controlled systems towards compliant, lightweight robots under impedance or interaction control [57]. If possible, mechanically: "The intrinsic or passive safety cannot be underestimated." [5] For instance, through the use of "soft actuators": series elastic or variable stiffness actuators that are back-drivable, also under control failure [153, 6]. Still, it is important to limit the robot's velocity to safe levels [57].

Returning to the injury criteria: many depend directly or indirectly on the energy transfer. The Maximum Power Index directly, but also for instance the strain and acceleration based measurements are related to energy. Control strategies that limit (mostly kinetic) energy present in the robot are successful: by estimating the energy in the robot, based on joint velocities, the position setpoint or maximum velocity are adapted to stay below unsafe energy levels $[149,80]$.

\section{$\S 1.1$ Passivity}

Better (that is, safer) than changing the robot's set point or scaling its velocity based on measured joint velocities, as in the referenced work above, is to ensure passivity of the robot. As proven by Stramigioli [140], if a system (the robot) is not strictly passive, it is always possible that a passive environment destabilises the system and extracts infinite energy from it. This is a very strong theoretical result: robots operating in an unpredictable environment can be guaranteed to remain stable if and only if they are passive.

Recently, Schindlbeck and Haddadin [125] presented a passivity-based control scheme for pHRI. Because most tasks do require energy, the authors introduced an task-based energy-tank method, where tasks can use a certain amount of energy but no more than that. 


\section{$\S 1.2$ Actuator-level passivity}

While the continuous-time control laws of Schindlbeck and Haddadin [125] are proven to be passive and the conducted experiments on a KUKA/DLR LWR-III were successful, what happens under coarse discretisation (of time and state measurements), (variable) time delays, or even communication loss between the high-level control computer and motor controllers in the joints?

Many modern control systems have a distributed architecture. If communication loss results in a failure of torque command updates to any of the low-level joint controllers, passivity cannot be guaranteed any longer. Therefore, we propose to move the passivity layer as close to the mechanical system as possible: if the actuators can be made passive, the whole system is always guaranteed to be passive [122]. Control messages will consist of the classical torque, velocity or position setpoint, combined with an energy budget, similar to the task-energy of [125].

In tele-manipulation and haptic interfaces, passivity has already been used to stabilise (variably) time-delayed systems, e.g. in the "PSPM" method of Lee and Huang [83], or PO/PC of Ryu, Kwon and Hannaford [118]. In these systems, the passivity relies on a series elastic element (PSPM) or precise interaction force measurements (PO/PC). In this paper, we present a method to add passivity to any electric-motor based actuator, without adding extra sensors.

\section{$\S 1.3$ This paper: $E^{2} A^{2}$}

This paper describes the idea of Embedded Energy-Aware Actuators $\left(\mathrm{E}^{2} \mathrm{~A}^{2}\right)$ : actuators that, on an embedded computing level, regulate the amount of energy they inject into the system. To illustrate the concept, the paper is structured as follows:

1. First, a very simple 1-DoF simulated system with passive continuoustime controller is presented. The demonstration system is discretised in such a way that it loses passivity, due to time delays. (Section C.2.2: The problem)

2. Exact energy measurement is implemented on the 1-DoF system, similar to $[146,19]$. (Section C.2.3: Energy sampling)

3. The controller is augmented with a local, discrete-time energy tank. (Section C.3: The $E^{2} A^{2}$ concept)

4. Two demonstration experiments are presented to show the guaranteed passivity and precise energy tank control in practice. (Section C.4: Experiments)

5. Finally, the method is applied to a $3-\mathrm{DoF}$ robot arm in simulation. (Section C.5: Robotic arm) 


\section{$\S 2$ Energy in controlled systems}

\section{§2.1 Passivity}

In physical systems, the property of passivity is an energy-based measure of stability - it is a special case of dissipativity as introduced by Willems [164] that arises naturally in physical dynamical systems. General dissipativity is defined in the following way: consider a system

$$
\dot{x}=f(x, w), \quad z=g(x, w)
$$

where $x$ is the system state, $w$ the input and $z$ the output, which take their values in their respective manifolds $X, W$ and $Z$. The supply function is a mapping

$$
s: W \times Z \rightarrow \mathbb{R}
$$

The system (C.1) is said to be dissipative if there exists a storage function $V: X \rightarrow \mathbb{R}$ such that

$$
V\left(x\left(t_{1}\right)\right) \leq V\left(x\left(t_{0}\right)\right)+\int_{t_{0}}^{t_{1}} s(w(t), z(t)) \mathrm{d} t
$$

The system is conservative if equality holds in (C.3). In physical systems, a natural choice for the storage function $V$ is the total energy in the system; the supply function is the supplied power $\langle F, v\rangle$ of the input port of the system. Indeed, systems that contain only passive physical elements (masses, springs, dampers) can never contain more energy than initially present. Consequently, the energy is bounded and thus a system that is overall passive is always stable $[95,122]$.

\section{$\S 2.2$ The problem}

Figure $C .1 \mathrm{a}$ is an example of a physical system with only an inertia $(1 \mathrm{~kg})$ and an actuator. Because the PD-controller acts as a virtual spring $\left(20 \mathrm{~N} \mathrm{~m}^{-1}\right)$ and damper $\left(0.1 \mathrm{~N} \mathrm{~s} \mathrm{~m}^{-1}\right)$, the overall system is passive and hence stable. When the controller is implemented in discrete-time, however, the system is not passive or stable anymore. Figure C.1b is the discrete-time implementation of the passive controller of Figure C.1a. Indeed, simulation results show that the discrete-time system becomes unstable, even at a rather high sampling frequency of $100 \mathrm{~Hz}$, for a system with an eigenfrequency of only $0.7 \mathrm{~Hz}$ (see Figure C.2).

Note that the original system was intentionally underdamped, and no special care was taken to do a proper discretisation, e.g. a bilinear transform. The $\mathrm{E}^{2} \mathrm{~A}^{2}$-method is completely independent of the chosen controller or system, so the purpose here is to obtain a simple demonstration system for the $\mathrm{E}^{2} \mathrm{~A}^{2}$-method. 


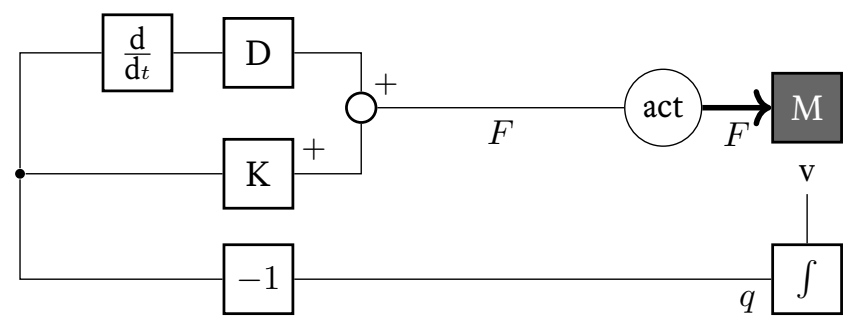

(a) Continuous-time controller

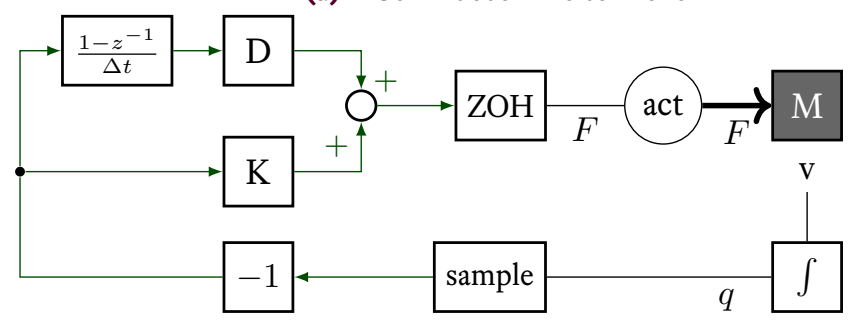

(b) Discrete-time controller. The sample block samples the position at each sample time; the ZOH (Zero Order Hold) block holds the previous force command during a sample timestep.

Figure $\mathbf{C} . \mathbf{1}$ - A very minimal system with a passive controller: a moving mass with a force actuator and a virtual spring/damper controller. 
Figure C.3 shows the energy present in the system, which decreases for the continuous-time, truly passive system, but grows for the non-passive discrete-time controller.

If instability due to time delays already occurs in this simple system, it is clear that we must take additional measures to ensure passivity-and thereby stability and safety - in any robotic or mechatronic system, especially if it has a distributed architecture, there is risk of communication loss, or is highly dynamic.

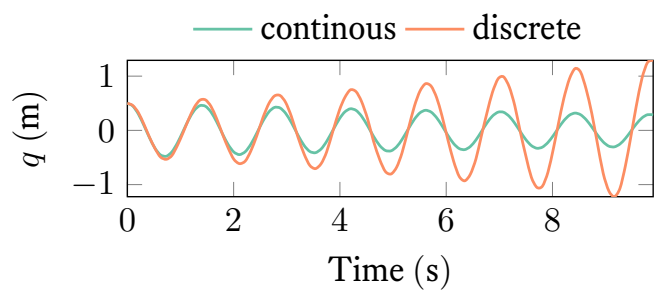

Figure C.2 - Comparison between a passive continuous-time controller and the same controller in discrete-time, with a sampling frequency of $100 \mathrm{~Hz}$. Notice that while the controller acts as a passive spring and damper, the discrete-time implementation is unstable.

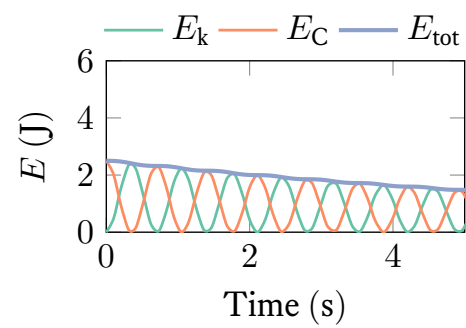

(a) Continuous-time system.

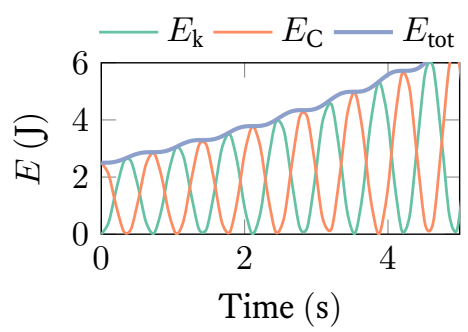

(b) Discrete-time system.

Figure C.3 - The energy present in the system and controller: kinetic energy $E_{k}$ in the mass and controller energy $E_{C}$ in the virtual spring. 


\section{$\S 2.3$ Energy sampling}

The first step towards ensuring passivity of the controller is measuring how much energy it supplies to the system. It is possible to accurately estimate the energy supplied by the controller if two conditions are met:

1. the actuator force is constant during a single sampling period;

2. there is a position sensor collocated with the actuator.

Condition 2 is a straightforward design choice; condition 1 is met if the actuator's inner control loop-e.g., the current controller of an electric motor-is sufficiently fast compared to the main control loop and a $\mathrm{ZOH}$ is used. When these conditions are met, the energy injected during a single sampling period can be calculated:

$$
\begin{aligned}
E & =\int_{t_{i}}^{t_{i+1}} P(t) \mathrm{d} t=\int_{t_{i}}^{t_{i+1}}\langle F(t), v(t)\rangle \mathrm{d} t \\
& =\int_{t_{i}}^{t_{i+1}}\left\langle F\left(t_{i}\right), v(t)\right\rangle \mathrm{d} t=\left\langle F\left(t_{i}\right), q\left(t_{i+1}\right)-q\left(t_{i}\right)\right\rangle
\end{aligned}
$$

Or, writing the discrete-time equations in computable form, where $\bar{F}_{i-1}$ is the control force set at $t_{i-1}$, held constant until $t_{i}$ by Condition 1 :

$$
\hat{E}_{i}=\hat{E}_{i-1}+\bar{F}_{i-1} \cdot\left(q_{i}-q_{i-1}\right)
$$

For the example system of Figure C.1b, this energy sampling method gives the result shown in Figure C.4: even at a very low sampling frequency of $25 \mathrm{~Hz}$, with a clearly unstable system, the energy is very accurately estimated.

Stramigioli et al. [146] introduced this energy sampling method as "sampled passivity" for discrete Port-Hamiltonian Systems, with a focus on stable and passive telemanipulation systems. The concept has until now only been used in the context of telemanipulation chains, not as a tool to build (distributed) passive architectures, other than in [19]. In this paper, for the first time this concept is used to make actuators intrinsically passive. In this new control paradigm, the actuator - as the interface between the continuous and discrete world-is responsible for ensuring passivity.

\section{$\S 3$ The $E^{2} A^{2}$ concept}

$\mathrm{E}^{2} \mathrm{~A}^{2}$ stands for "Embedded Energy-Aware Actuators": by embedding the energy sampling as explained in the previous section in an actuator, or as close to it as possible, the actuators themselves can keep track of the 


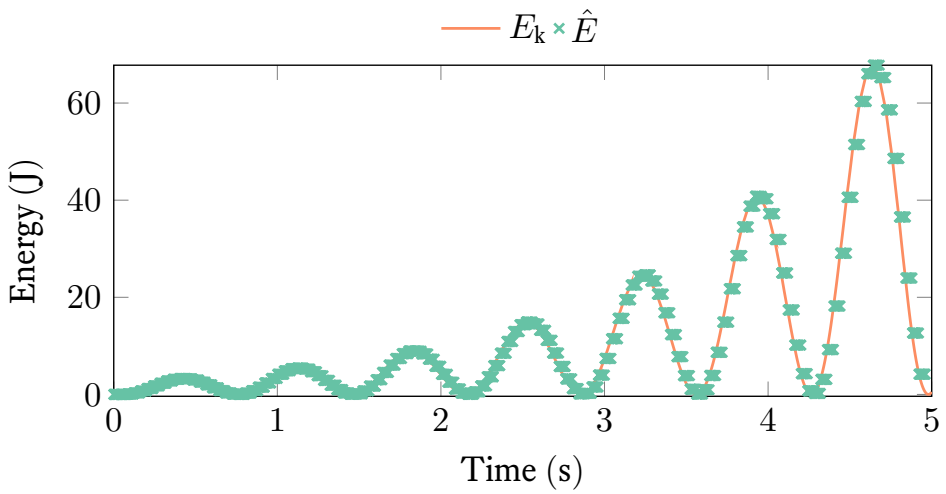

Figure C.4 - Supplied energy estimated by "passive sampling", compared to the actual kinetic energy of the mass. To show the effectiveness, sampling frequency has been reduced to $25 \mathrm{~Hz}$.

energy that they inject into-or extract from - the mechanical system or robot. To ensure passivity of the actuator, two more things are required: an energy tank, and a strategy to apply when the tank is empty.

\section{$\S 3.1$ The energy tank}

By providing the actuator with an initial energy $E_{0},(\mathrm{C} .5)$ can be used to keep track of an "energy tank": any energy the actuator supplies to the robot must be taken from this tank. Hence, when the tank is empty, the actuator's power output must be zero: passivity is then guaranteed. Note that any negative work done by the actuator also "flows back" into the tank, since injected energy $F \Delta q$ of (C.5) is negative.

The injection of energy by the actuator into the mechanical part of the robot can be forced to 0 in two ways: forcing $F$ to zero, or forcing $v$ to zero. Which of these is the proper choice depends very much on the system: setting $F$ to zero still allows other (external) forces on the system to accelerate the robot and actuator. If gravity is present in the system, it is better to force $v$ to zero (constant position). On the other hand, in the mass-spring-damper system the logical choice is $F=0$.

Actually, when $E=0$ the actuator must not inject additional energy into the system; it may of course extract energy from it. The $\mathrm{E}^{2} \mathrm{~A}^{2}$-type actuator has an embedded passivity layer between controller and actuator 


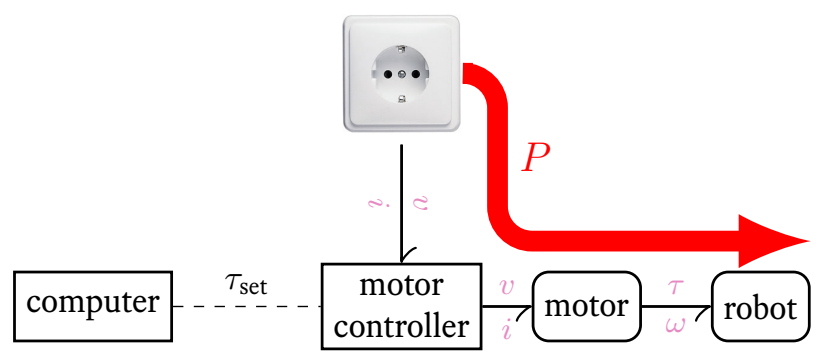

(a) Classical actuators have an unlimited power supply and can therefore inject unlimited energy into the system.

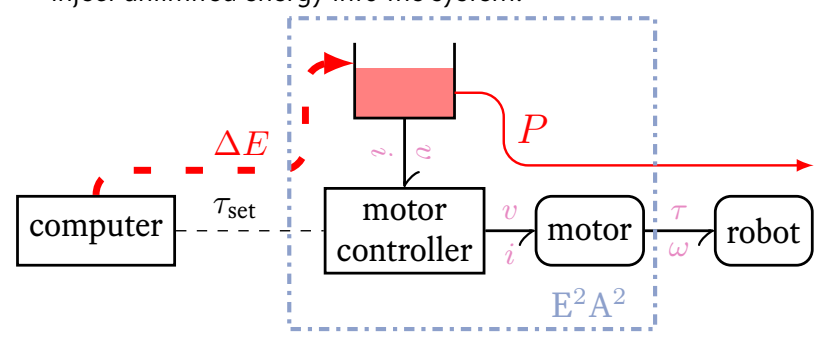

(b) $E^{2} A^{2}$-type actuators have a virtual energy tank and can therefore never inject more energy into the system than the amount that was provided explicitly by the controller.

Figure C.5 - Classical actuators versus Embedded Energy-Aware Actuators. Although both are connected to an infinite power supply and might lose communication with the computer, the $E^{2} A^{2}$ has a virtually limited amount of energy available. 
that "passivates" the system as follows:

$$
F_{\text {actuator }}=\left\{\begin{array}{lll}
F_{\text {controller }} & & \hat{E}>0 \\
0 & F_{\text {controller }} \cdot \hat{\dot{q}} \geq 0 & \hat{E} \leq 0 \\
F_{\text {controller }} & F_{\text {controller }} \cdot \hat{\dot{q}}<0 &
\end{array}\right.
$$

Note that for (C.6) an estimate of the actuator velocity direction sgn $(\dot{q})$ is required.

\section{$\S 3.2$ The new "set-points"}

With the new Embedded Energy-Aware Actuators in place, a control message sent over the system bus is not longer just a set-point for torque, velocity or position, but also includes the energy that may be expended during the motion: $\sigma:=(r, \Delta E) . \Delta E$ is added to the local energy tank; $r$ is executed until the next set-point $\sigma$ comes in, or until the energy tank is empty.

With task-based energy budgets, a controlled amount of energy may be injected or "used" by the actuator. The budget may depend on expected potential energy change (when lifting a robot arm), or e.g. friction losses.

\section{$\S 4$ Experiments}

Two different experimental setups were constructed to show the working of the $\mathrm{E}^{2} \mathrm{~A}^{2}$ in practice: the running example of an inertia with an actuator that implements a spring; and a motor with a pulley that lifts a mass (see Figure C.6). The former clearly shows instability in oscillations, which is solved by the new actuator; the latter neatly demonstrates the accuracy of the energy estimate, which should of course be approximately equal to the mass's potential energy, in turn proportional to its height.

The electric motors of both setups are driven by a motor controller ${ }^{1}$ operating in current control mode, which also runs the energy tank algorithm. A computer, communicating with the motor controller over an $\mathrm{RS}_{232}$ serial connection, sets the torque/current command and available energy.

${ }^{1}$ Elmo SimplIQ Solo Whistle. 


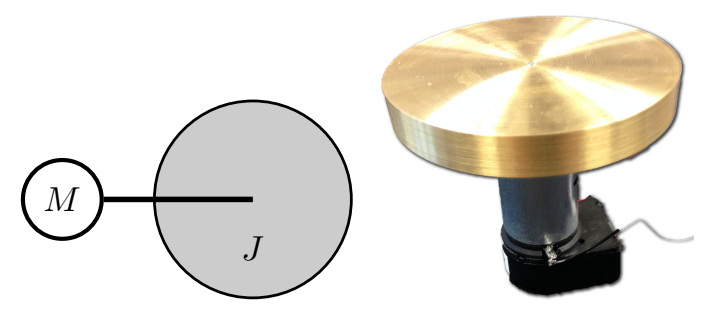

(a) A motor $M$ that acts as a virtual rotational spring for a large inertia $J$.

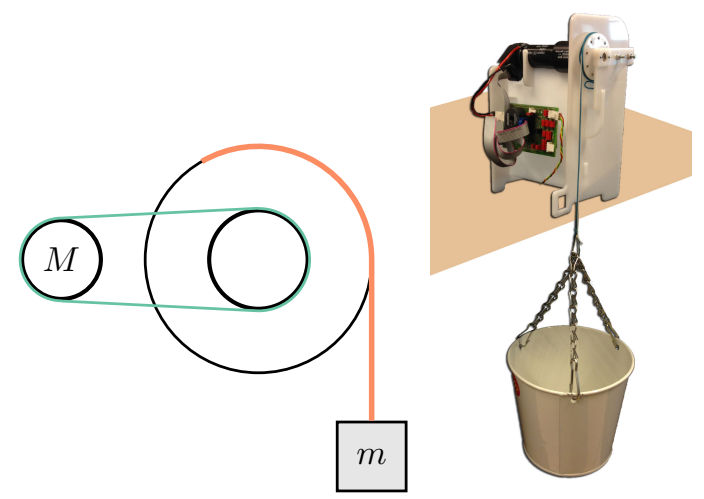

(b) A motor $M$ that lifts a mass $m$ by means of a pulley.

Figure C.6 - Systems used for testing the $E^{2} A^{2}$ architecture. 


\section{$\S 4.1$ Virtual spring}

The very simple experimental setup illustrated in Figure C.6a consists of an electric motor ${ }^{2}$ with a heavy brass disk bolted directly to the motor shaft. The control algorithm on the computer implements a spring with a stiffness of $3.98 \mathrm{mN} \mathrm{m} \mathrm{rad}^{-1}$, which results in an eigenfrequency of $0.51 \mathrm{~Hz}$ with the disk's inertia of $3.76 \times 10^{-4} \mathrm{~kg} \mathrm{~m}^{2}$. The algorithm runs at $50 \mathrm{~Hz}$.

\section{Regular actuator}

First, the experiment is carried out without the embedded passivity layer: the controller is started and the disk given a small initial push to start oscillation. Figure C.7 shows that the disk quickly spins out of control and the motor keeps injecting energy into the system. Clearly, also a very simple system will quickly become unstable; even when the sampling frequency is two orders of magnitude higher than the eigenfrequency.

\section{$\mathrm{E}^{2} \mathrm{~A}^{2}$-actuator}

With the $\mathrm{E}^{2} \mathrm{~A}^{2}$-concept implemented, the low-level controller keeps track of injected energy; the computer includes the initial energy tank contents $(0.1 \mathrm{~J})$ in the first message. Now, the passive controller is indeed passive: whenever the tank is empty, the current (torque) is cut off to $0 \mathrm{~A}$, preventing further energy injection. The disk keeps oscillating as expected.

\footnotetext{
${ }^{2}$ maxon A-max 26, 110201
} 


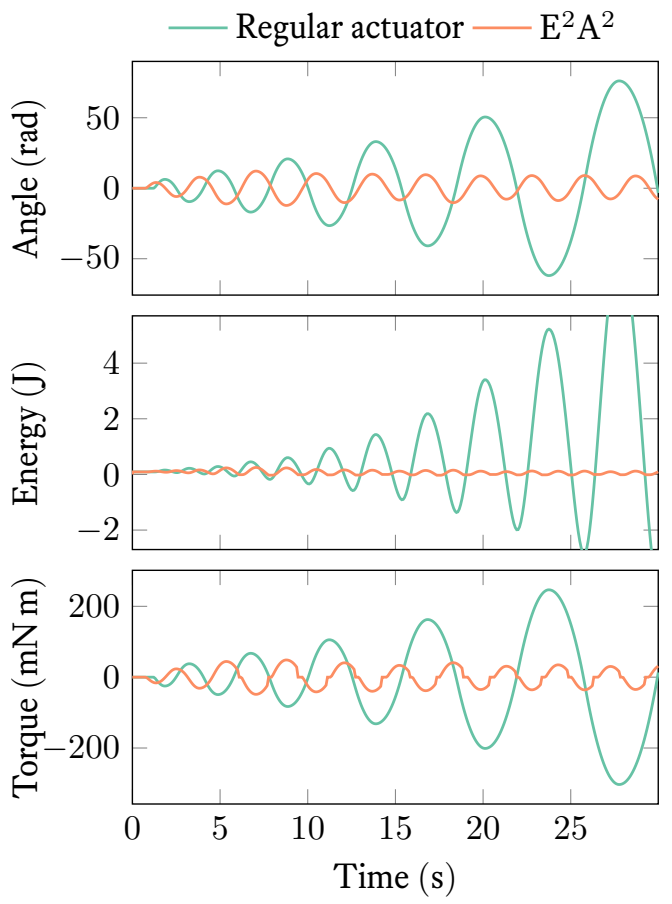

Figure C.7 - Experimental results for the virtual spring setup depicted in Figure C.6a. Note that the torque is continually cut off for the passivated system when the energy tank is empty, resulting in a stable system with bounded energy. 


\section{$\S 4.2$ Lift}

The lift experiment consists of a motor with gearbox ${ }^{3}$ and a pulley, which lift a mass - see Figure C.6b for a schematic drawing. The control command will be a constant torque that lifts the weight off the ground, which requires an amount of energy equal to the potential energy gained by the mass. Note that instead of a constant torque, any torque or even velocity profile can be applied-the $\mathrm{E}^{2} \mathrm{~A}^{2}$-method works independently from high-level control law and the actual system. In this case when the energy tank is empty, the torque cannot be set to $0 \mathrm{~N} \mathrm{~m}$ : the mass would drop to the floor. Instead, a weak P-controller is engaged locally at the actuator to hold the mass steady. If extra energy is added to the tank by a control message or by pulling the mass down (against the P-controller, which extracts energy from the environment), the actuator will switch back to the original torque command.

Figure C.8a shows the experimental results: indeed, the mass is halted shortly before the motor has rotated $400 \mathrm{rad}$, because the energy tank has been emptied. With a gearbox ratio of $33: 1$ and pulley radius of $2 \mathrm{~cm}$, this corresponds to a height of $23 \mathrm{~cm}$. The mass weighs $816 \mathrm{~g}$, so the potential energy gain is only about $1.8 \mathrm{~J}$-apparently a lot of the controller energy of $3 \mathrm{~J}$ is lost to friction.

In order to verify this, we have modelled the system with the modelling and simulation program 20-sim [29]. The parameters that were used are listed in Table C.1. The mechanical friction is implemented as a Coulomb-viscous friction model according to (C.7). These parameters were fitted to the measurements to obtain the results shown in Figure C.8b. This simulation confirms that $1.2 \mathrm{~J}$ is lost in motor/gearbox friction.

$$
\tau_{R}=\tau_{\text {Coulomb }} \cdot \operatorname{sgn}(\omega)+R_{\text {viscous }} \cdot \omega
$$

\section{Friction loss compensation}

With the energy loss estimate, we could carefully add extra energy to the tank and achieve the desired height of $35 \mathrm{~cm}$ - at the risk of overshooting if the actual friction is, after all, less than the modelled friction. First, the simulation was used to estimate the energy lost to friction when the mass is pulled all the way up to $35 \mathrm{~cm}$, which turned out to be $1.8 \mathrm{~J}$. When this energy was added to the tank at the start of the experiment, on top of the original $3 \mathrm{~J}$, the mass does indeed reach the intended height of $35 \mathrm{~cm}$.

\footnotetext{
${ }^{3}$ maxon DC motor 118746, maxon gear 166938.
} 


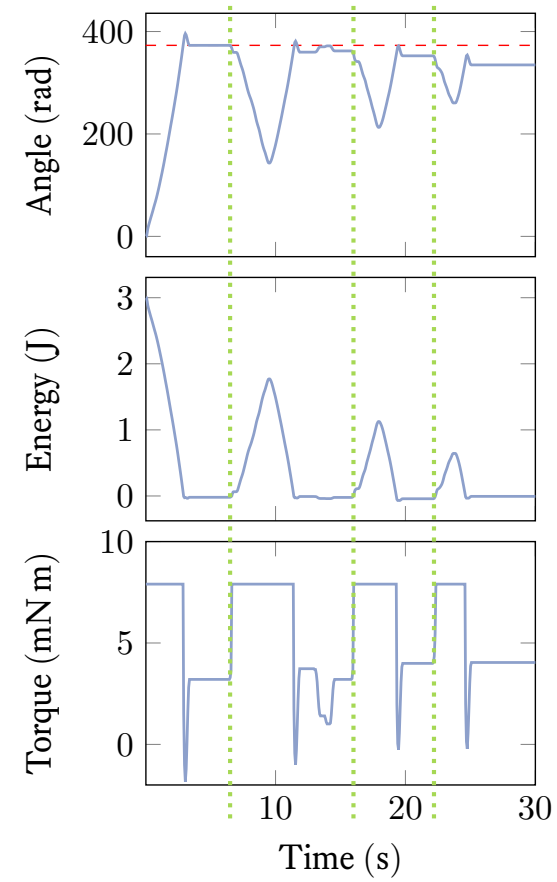

(a) Experiment.
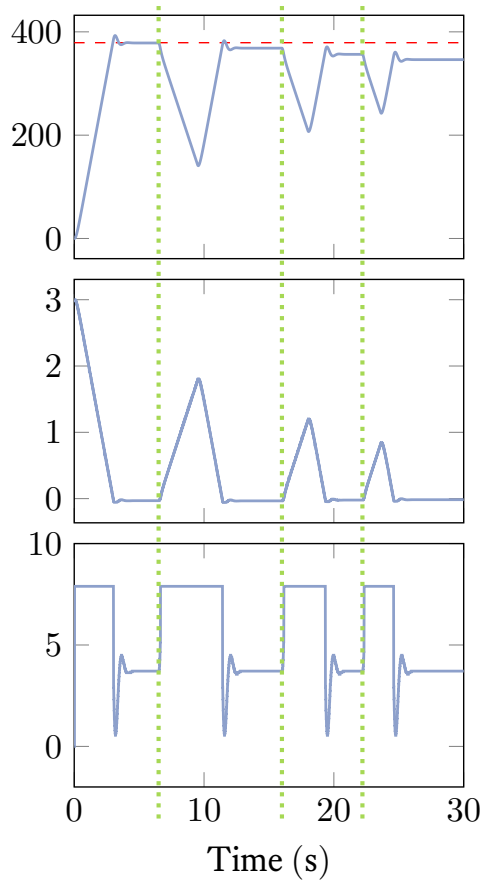

(b) Simulation.

Figure C.8 - Lift simulation and experimental results. The mass is pulled downwards at $6.5,16$ and $22.5 \mathrm{~s}$ (which injects energy into the actuator) and lifted at $t=12 \mathrm{~s}$ (which does nothing: the cable slacks). The red, dashed line shows that a little energy is lost to friction every time the mass moves down and up.

\begin{tabular}{lclr}
\hline Parameter & \multicolumn{2}{c}{ Value } & Description \\
\hline$K_{m}$ & 43.9 & $\mathrm{mN} \mathrm{m} \mathrm{A}^{-1}$ & Motor constant \\
$\tau_{\text {Coulomb }}$ & $3.0 \times 10^{-5}$ & $\mathrm{Nm}$ & Coulomb friction \\
$R_{\text {viscous }}$ & $4.0 \times 10^{-5}$ & $\mathrm{Nm} /(\mathrm{rad} / \mathrm{s})$ & Viscous friction \\
$J_{\text {motor }}$ & $10.5 \times 10^{-7}$ & $\mathrm{~kg} \mathrm{~m}^{2}$ & Motor inertia \\
$n$ & $1: 33$ & & Gearbox ratio \\
$J_{\text {gearbox }}$ & $8.7 \times 10^{-5}$ & $\mathrm{~kg} \mathrm{~m}^{2}$ & Gearbox inertia \\
$r$ & 0.020 & $\mathrm{~m}$ & Pulley radius \\
$m$ & 0.816 & $\mathrm{~kg}$ & Mass \\
$F_{g}$ & -8.0 & $\mathrm{~N}$ & Weight \\
\hline
\end{tabular}

Table C.1 - Parameters for the lift simulation. 


\section{$\S 5$ Robotic arm}

We have carried out a simulation of a $3-\mathrm{DoF}$ robot arm with and without the use of Embedded Energy-Aware Actuators. Although this is a computer simulation, the previous experiments have shown that the $\mathrm{E}^{2} \mathrm{~A}^{2}$ approach works in practice, guaranteeing passivity or precise control of the amount of injected energy. This experiment serves to demonstrate the application of the $\mathrm{E}^{2} \mathrm{~A}^{2}$-method for energy safety guarantees on a more complicated system.

\section{§5.1 System and Controller}

The arm consists of three links and three rotational joints (see Figure C.9). In the reference configuration $\left(q_{1}=q_{2}=q_{3}=0\right)$ the joint axes are $z$, $y$ and $x$, respectively. Each link is $0.5 \mathrm{~m}$ long and weighs $1 \mathrm{~kg}$; a uniform weight distribution is used. Each joint is modelled as a torque actuator with a viscous friction of $2.0 \mathrm{~N} \mathrm{~m} \mathrm{~s} \mathrm{rad}^{-1}$.

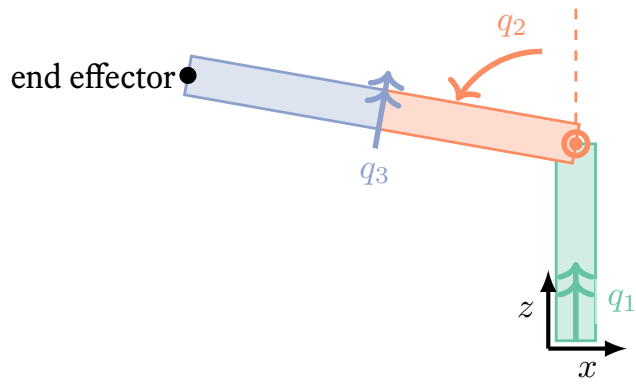

Figure C.9 - The robotic arm consists of three links with three rotational joints. Each link is $0.5 \mathrm{~m}$ long and weighs $1 \mathrm{~kg}$. When $q_{i}=0, \forall i$, the arm points straight up.

The controller is impedance control on the end-effector, with stiffness $K=20 \mathrm{~N} \mathrm{~m}^{-1}$ and with active gravity compensation according to (C.9). $\tau$ is the torque sent to the joint actuators and $\tau_{\text {gravity }}$ is the calculated joint torque due to gravity. The Jacobian $J$ of (C.8) is derived from the robot kinematics. $\mathbf{x}$ is the $\left(\begin{array}{lll}x & y & z\end{array}\right)^{\top}$ position of the end-effector or setpoint; $\mathbf{q}$ are the joint positions $\left(\begin{array}{lll}q_{1} & q_{2} & q_{3}\end{array}\right)^{\top}$.

$$
\begin{aligned}
\mathbf{v}_{\mathrm{ee}} & =J(\mathbf{q}) \cdot \dot{\mathbf{q}} \\
\tau_{\text {impedance }} & =J(\mathbf{q})^{\top} \cdot K\left(\mathbf{x}_{\text {setpoint }}-\mathbf{x}_{\mathrm{ee}}\right) \\
\tau & =\tau_{\text {impedance }}-\tau_{\text {gravity }}
\end{aligned}
$$




\section{$\S 5.2$ Experiment}

The control law of (C.10) is implemented in discrete-time, with a frequency of $100 \mathrm{~Hz}$, and simulated 12-bits angular encoders on each joint. The actuators are implemented as Embedded Energy-Aware Actuators from the lift experiment.

At the start of the simulation, the arm starts in its zero configuration (straight up). At $t=0 \mathrm{~s}$ the setpoint is $\left(\begin{array}{lll}0.3 & 0.4 & 1.0\end{array}\right)^{\top} \mathrm{m}$; at $t=5 \mathrm{~s}$ it changes to $\left(\begin{array}{lll}-0.7 & 0.1 & 1.2\end{array}\right)^{\top} \mathrm{m}$.

The experiment is carried out twice: once without the energy-limit (the torque from (C.10) is sent to the motors directly) and once with the $\mathrm{E}^{2} \mathrm{~A}^{2}$-scheme in place at each actuator. Because there is a significant amount of potential energy change from the first to the second setpoint, each actuator is given an energy budget of $4 \mathrm{~J}$ in the second experiment.

\section{$\S 5.3$ Results}

The left half of Figure C.10 shows the results of the first experiment. The end-effector is pulled towards the setpoint by the impedance controller. Due to the large distance between setpoint 1 and 2, the kinetic energy reaches high levels during that change (middle left: $12 \mathrm{~J}$ ). In total, the actuators inject almost $20 \mathrm{~J}$.

In the second experiment, right of Figure C.10, the $\mathrm{E}^{2} \mathrm{~A}^{2}$ limits the total amount of energy injected (bottom right), which also keeps the maximum kinetic energy bounded. The velocity of the arm is much decreased and the setpoint is reached much slower than before, as seen in the top right plot. This is of course a direct effect of the desired lower kinetic energy. 

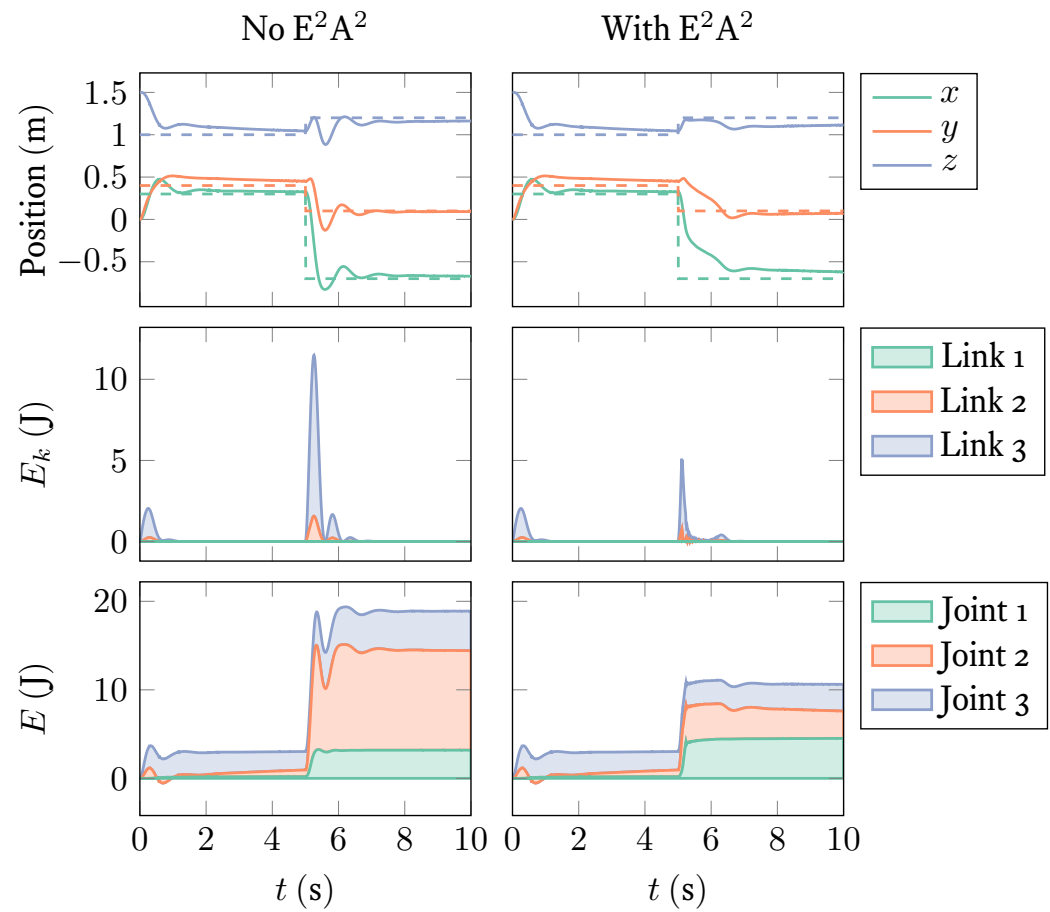

Figure C.10 - Robot arm simulation experiment without (left) and with (right) $E^{2} A^{2}$ : during a large setpoint change, the kinetic energy reaches high levels. Note that the energy plots are stacked. 


\section{$\S 6$ Conclusion}

Robots in unknown environments, especially when there is physical Human-Robot Interaction, benefit from precise control over the amount of energy present in the physical system. Indeed, passivity is a necessary requirement for stability [140].

In modern distributed architectures, where the-possibly passivecontrol algorithms send torque or position commands to low-level motor controllers, communication malfunctioning will likely render the system unstable. For sure, without extra precautions, passivity cannot be guaranteed any longer.

In this paper we have presented $\mathrm{E}^{2} \mathrm{~A}^{2}$ : energy-awareness and passivity, or exact control over the amount of injected energy, at the actuator level. Even in the case of communication failure between high-level controller and motor controllers, passivity and stability are still guaranteed.

Embedded Energy-Aware Actuators are a model-free safety and stability layer, realised by:

1. Defining an "energy budget" that may be used by an actuator;

2. keeping track of the amount of energy injected into the system by the actuator;

3. taking measures to avoid injecting additional energy when the whole budget has been used.

By implementing this passivity layer locally at the actuator, so by embedding it in the low-level controller, overall system passivity and stability is guaranteed-even under communication failure conditions. The actuators themselves have become energy-aware.

Two experiments have shown that implementing the $E^{2} \mathrm{~A}^{2}$-concept ensures passivity and stability. A simulation experiment has demonstrating the effect of applying the $\mathrm{E}^{2} \mathrm{~A}^{2}$-method on a more complicated system: a 3-DoF robotic arm.

In today's complex robotic systems, it is worthwhile to think about the energy in the system: for stability and, most importantly, safety. With Embedded Energy-Aware Actuators, it is possible to ensure adherence to an energy budget - to prevent control malfunctions, system damage and injuries. 


\section{Dankwoord}

Thank you.

Yes, you, the reader. The fact that you're reading this means that at least someone is appreciating the work I put into writing this thesis - or at least into these acknowledgements. * Furthermore, it probably also means that you are at my defence, a colleague, or in some other way involved in my research. So, again: thank you, dear reader.

Dan door naar het echte dankwoord. Ik heb getwijfeld of ik iedereen zou proberen te benoemen - met het risico iemand te vergeten - of te volstaan met een "iedereen bedankt." (Uiteindelijk weten degenen die een bijdrage hebben geleverd en mijn dank verdienen dat toch wel.) Maar ik blader zelf bij andere proefschriften ook altijd naar het dankwoord en weet hoe leuk het is om je eigen naam daarin te ontdekken, dus we gaan toch voor optie 1 . Kun je je naam niet vinden in het volgende, ga dan niet zitten pruilen: ofwel ik ben je vergeten, waarvoor mijn excuses; ofwel je hebt toch minder bijgedragen dan je dacht. Kies zelf maar.

Ten eerste mijn promotor en "dagelijks begeleider" Stefano. Er waren momenten dat het beperkte contact tussen ons lastig was, maar over het algemeen heb ik heel veel gehad aan je vertrouwen, inspiratie en input tijdens inhoudelijke discussies. Door de grote vrijheid en verantwoordelijkheid die je me gaf, heb ik me kunnen ontwikkelen - niet alleen op wetenschappelijk gebied, maar juist ook daarbuiten. Mijn co-promotor was Arjan van der Schaft, van de RuG: zo af en toe maakte ik een tripje naar Groningen, of kwam Arjan naar Twente. We hebben niet intensief samengewerkt, maar juist daardoor kon je altijd met een kritische blik naar mijn onderzoek, artikelen en plannen kijken. Vooral je inhoudelijke input op de wat meer wiskundige zaken was zeer waardevol. Stefano, Arjan: bedankt!

In een vakgroep is veel verloop van studenten en aio's, maar met degenen die ongeveer in hetzelfde cohort zitten werk je toch gauw 6 jaar samen. Douwe: er is te veel om alles op te noemen. Lopende robots, activiteiten voor de brainstorm, discussies over wetenschap, auto
* If you haven't done so, I recommend reading at least the Samenvatting or Summary, which give a good idea of the full contents of this thesis. 
delen, ervaringen uitwisselen over kinderen, werken aan de organisatie, aanzetten tot OFL, .... Ik ben blij dat wij in hetzelfde cohort zaten!

Éamon, ook wij begonnen ongeveer tegelijk. We waren te veel met onze eigen projecten bezig om veel samen te werken, maar ik heb meermalen dankbaar gebruik gemaakt van je werktuigbouwkundige kennis en inzicht, en jou hopelijk verder geholpen met geometrische vraagstukken.

In de ruim vier jaar die ik aio was bij RaM, heb ik veel studenten mogen begeleiden: het is niet voor niets dat twee hoofdstukken uit dit proefschrift direct voortgekomen zijn uit het werk van afstudeerders. In chronologische volgorde: Eric (glide mode v2), Wessel (autopilot), Ivor (hoppers), Cyrano (vleugelslagopstelling), Rik (parachute), Berend (vleugelslag), Bart (cheetah), Luca (e2a2), Frank (robotarm), Frank (robirdmodel), Jeroen vD (glide mode v3), Erik (robird-opstelling), Jeroen M (robird-mechanisme), Ernesto (glide mode v3), Nicolò (cheetah), Alan (lanceerplatform), Camiel (fasesturing), Martijn (hopper v3), Steven (vleugelslag), Jelmer (landingsplatform).

Bedankt voor jullie bijdragen aan mijn onderzoek!

Bij het ontwikkelen van robots hoort ook het bouwen van robots. Ikzelf, maar vooral de hiervoor genoemde studenten, zijn hierbij altijd enorm geholpen door de technici van RaM. Zonder de ervaring, hulp en parate kennis van Gerben was het rapid prototyping niet gelukt; alle RaMstix-perikelen werden door Marcel verholpen. Ook Hennie kon me af en toe helpen bij een mechanisch vraagstuk. Heren: bedankt!

Uit het feit dat ik al sinds mijn bacheloropdracht in 2009 bij RaM rondloop, blijkt dat ik het wel naar mijn zin heb in deze vakgroep. Dat komt misschien een beetje doordat ik er mijn onderzoek kan doen, maar toch vooral door de mensen. De vaste staf (met wat wisselingen in de wacht door fusies en splitsingen), ondersteuning (ik geloof dat ik je eerste BSc-student was toen je bij CE kwam, Jolanda), aio's en studenten

Een bijzonder woord van dank aan Peter Breedveld, die zo diep wist te gaan bij colleges over dynamisch modelleren dat ik het maar net kon volgen. die komen en gaan - samen maken ze de groep. Hoogtepunten waren de oprichting van de StiBiBaRo met Jos Vos en het aanbrengen van ledverlichting in de koffieruimte.

Ik heb het geluk gehad om vrijwel vanaf de oprichting bij Clear Flight Solutions betrokken te zijn en zo de ontwikkeling van de vogel, van prototype tot product, mee te maken en er mijn steentje aan bij te dragen. Inmiddels werk ik er officieel en ik hoop samen met het geweldige team daar nog veel verder te komen met de vogels!

Mijn interesse in de wetenschap en robotica is voor een groot deel gevormd door inspirerende docenten. Van Guit-Jan Ridderbos die mij als welpje tijdens een hike $2^{12}$ liet uitrekenen, via Meile Haan en Jan Bos die mij op de middelbare school wisten uit te dagen, tot professors als Ruud van Damme, Gijs Krijnen en Leon Abelmann, die allemaal op hun beurt net iets méér deden dan gewoon college geven. Ik hoop dat jullie blijven inspireren. 
Buiten mijn promotie om is natuurlijk veel meer gebeurd dan erbinnenper slot van rekening zit er van de 168 uur in de week slechts 40 in de werkweek. Idealiter nog zo'n 56 in slapen, maar zelfs als dat gehaald wordt dan blijft er 72 uur over. Die tijd is voor familie, vrienden en hobby's.

In omgekeerde volgorde: aan alle muzikanten van Excelsior Westenholtein het bijzonder aan de sexy sectie natuurlijk - en aan Marten hoef ik niet te vertellen hoezeer ik het er naar mijn zin heb; dat ik er elke donderdag voor naar Zwolle reis spreekt boekdelen.

Ontwikkelingen in vestigingsplaats en gezinsleven zorgen dat de kwantiteit minder wordt, maar de kwaliteit is dik in orde: Auke, Hein, Ramon, Bas, ik hoop dat de kwantiteit nooit 0 wordt. Ook andere vrienden, van school, studie en daarbuiten: zonder jullie was het minder.

Ten slotte de familie. Die kies je niet (nou ja, de helft misschien), dus je moet het geluk hebben dat het botert. Dat heb ik. Heit, mama, Anne, Jelte; Leendert, Menne: ik kijk alweer uit naar Terschelling! En mijn favoriete tante in Frankrijk, waar ik een paar jaar achter elkaar flink kon meeklussen, en natuurlijk eten $\&$ drinken. Ook de andere kant (die ik dus min of meer wel heb gekozen) zit wel snor: Ruud, Jo, Marloes, Carlijn; Stefan; José: ik zou me een vervelender schoonfamilie kunnen voorstellen.

Mijn vrouw heeft me op niet mis te verstane wijze duidelijk gemaakt dat het dankwoord toch vooral een pagina's-tellende lofzang aan de partner moet zijn. Nu houd ik nauwlettend de git-repository van háár proefschrift in de gaten, maar zie daar nog niet iets wat dergelijke proporties aanneemt. Vanuit het gelijkheidsbeginsel denk ik dus dat ik wel weg kan komen met een iets korter stukje. Thanks, Laura!

Last of all, then. These may be the acknowledgements of my thesisand I don't think you two had any positive contribution to that - but still: Iris and Jasper, you are a delight. (If only because the sleeping time mentioned above is lowered drastically, putting even more "awake" hours in a week.) Thanks a lot!

26 en $\mathrm{D}+\mathrm{K}$ : bedankt voor jullie onvoorwaardelijke steun, zeker na de geboortes van IEJ.

Kijk, ik kan hier wel een heel uitgebreid verhaal gaan houden, maar het wordt ook op internet gepubliceerd en straks wordt het nog gênant. Ik hou van je. 


\section{About the author}

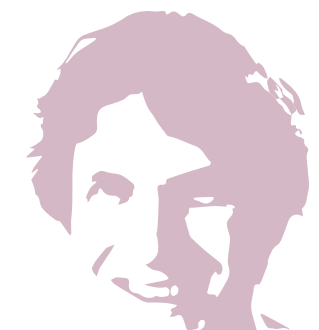

Geert Folkertsma was born in Westenholte, Zwolle, in the spring of 1988. He went to school at the Meander College, studying a broad range of courses including Physics, Chemistry, Music, Economy, Mathematics, Drama. Still, the STEM subjects interested him most of all, participating in the "elektronicaclub" and two National and International Chemistry Olympiads.

The bachelor's programme "Advanced Technology" (BSc 2010, cum laude) and master's programme "Mechatronics" (MSc 2012, cum laude) were both followed at the University of Twente, with an internship at the Biomimetics Robotics Lab, Massachusetts Institute of Technology. Geert was made Member of Merit of the study association for Advanced Technology, Astatine, for his active involvement with the association.

During the last year of his MSc programme, Geert obtained funding from the Dutch Institute for Systems and Control for a PhD research project on quadrupedal running, "FREEQ." During the four-and-a-half years of this project he not only focussed on the research and obtaining the DISC course certificate, but supervised many students and taught various courses at BSc and MSc level.

After cooperating with Robird-spinoff "Clear Flight Solutions" for four years, winning the European Robotics Forum 2016 Technology Transfer Award with the team, since 2016 Geert has been employed part-time at CFS and part-time at the UT. At the time of writing, he is employed at the university as an assistant professor and at the Robird company as lead Research \& Development engineer. Geert is currently living in Enschede with his wife and their two children. 